THREE-DIMENSIONAL (3-D) IMMERSED-BOUNDARY (IB) BASED

TORNADO MODEL FOR COMPUTATIONAL ANALYSIS OF

DISASTROUS WIND LOAD ON BUILDINGS

by

Xixiong Guo

Master of Applied Science, Mechanical Engineering, Ryerson University, Toronto, Canada, 2014

Bachelor of Engineering, Northwestern Polytechnical University, Xi’an, China, 2011

A dissertation
presented to Ryerson University

in partial fulfilment of the

requirements for the degree of

Doctor of Philosophy

in the program of

Mechanical \& Industrial Engineering

Toronto, Ontario, Canada, 2019

(C) Xixiong Guo, 2019 


\section{Author's Declaration}

I hereby declare that I am the sole author of this dissertation. This is a true copy of the dissertation, including any required final revisions, as accepted by my examiners.

I authorize Ryerson University to lend this dissertation to other institutions or individuals for the purpose of scholarly research.

I further authorize Ryerson University to reproduce this dissertation by photocopying or by other means, in total or in part, at the request of other institutions or individuals for the purpose of scholarly research.

I understand that my dissertation may be made electronically available to the public. 


\title{
THREE-DIMENSIONAL (3-D) IMMERSED-BOUNDARY (IB) BASED \\ TORNADO MODEL FOR COMPUTATIONAL ANALYSIS OF \\ DISASTROUS WIND LOAD ON BUILDINGS
}

\author{
Doctor of Philosophy, 2019 \\ Xixiong Guo \\ Department of Mechanical and Industrial Engineering \\ Ryerson University
}

\begin{abstract}
This study is aimed at developing a novel computational framework that can essentially simulate a tornadic wind field and investigate the wind loadings on ground constructions. It is well known that tornado is a highly turbulent airflow that simultaneously translates, rotates and updrafts with a high speed. Tornadoes induce a significantly elevated level of wind forces if compared to a straight-line wind. A suitably designed building for a straight-line wind would fail to survive when exposed to a tornadic-like wind of the same wind speed. It is necessary to design buildings that are more resistant to tornadoes. Since the study of tornado dynamics relying on field observations and laboratory experiments is usually expensive, restrictive, and time-consuming, computer simulation


mainly via the large eddy simulation (LES) method has become a more attractive research direction in shedding light on the intricate characteristics of a tornadic wind field.

For numerical simulation of a tornado-building interaction scenario, it looks quite challenging to seek a set of physically-rational and meanwhile computationally-practical boundary conditions to accompany traditional CFD approaches; however, little literature can be found, as of today, in three-dimensional (3D) computational tornado dynamics study. Inspired by the development of the immersed boundary (IB) method, this study employed a re-tailored Rankine-combined vortex model (RCVM) that applies the "relative motion" principle to the translational component of tornado, such that the building is viewed as "virtually" translating towards a "pinned" rotational flow that remains time-invariant at the far field region. This revision renders a steady-state kinematic condition applicable to the outer boundary of a large tornado simulation domain, successfully circumventing the boundary condition updating process that the original RCVM would have to suffer, and tremendously accelerating the computation.

Wind loading and its influence factors are comprehensively investigated and analyzed both on a single building and on a multiple-building configuration. The relation between the wind loadings and the height and shape of the building is also examined in detail. Knowledge of these loadings may lead to design strategies that can enable ground construction to be more resistant to tornadoes, reducing the losses caused by this type of disastrous weather. 


\section{Acknowledgements}

Firstly, I would like to express my sincere gratitude to my supervisor Prof. Jun Cao for giving me the opportunity to work on this exciting project and for his constant support and valuable guidance during this study. In the past five years, his diligence to research, rigid attitude toward scholarship, and many other characteristics influenced me strongly. He cared so much about my work, and always responded to my questions and queries so promptly.

Besides my supervisor, I would like to thank the rest of my thesis committee: Prof. Ziad Saghir, Prof. Wey Leong, Prof. Joon Chung, and Prof. Dong Liang for their efforts to serve as the committee members and for their insightful comments and suggestions.

I would also like to thank my girlfriend, $\mathrm{Xi}$, for encouraging me throughout this $\mathrm{PhD}$ journey. She always stands by me through all the good and bad times.

Last, I would like to thank my parents for their love and encouragement. They have always been there for me, supported me, and believed in me

This work is financially supported by Dr. Cao's Discovery Grant awarded by the Natural Sciences and Engineering Research Council (NSERC) of Canada. 


\section{Table of Contents}

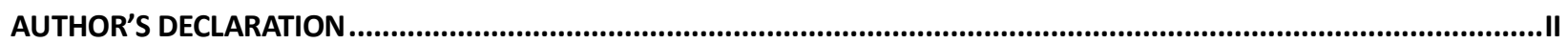

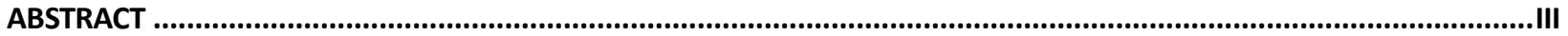

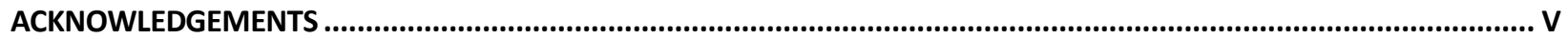

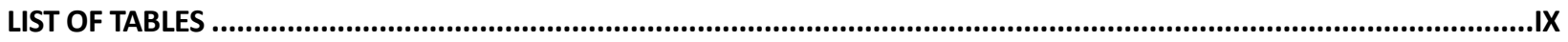

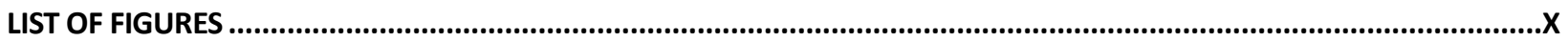

NOMENCLATURE

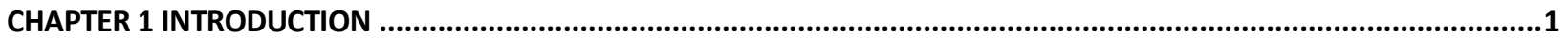

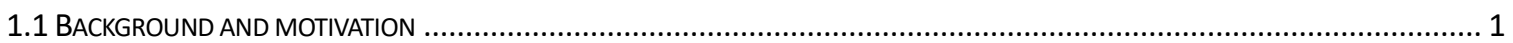

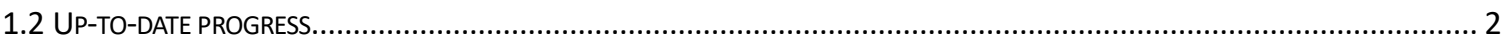

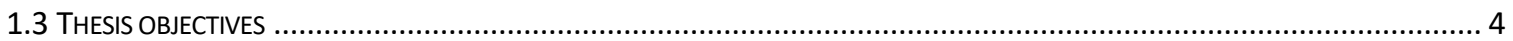

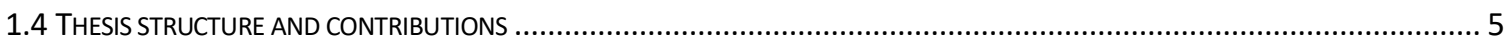

CHAPTER 2 AN IB-LBM INVESTIGATION INTO THE AERODYNAMIC COEFFICIENTS IN RELATION TO THE ROTATION

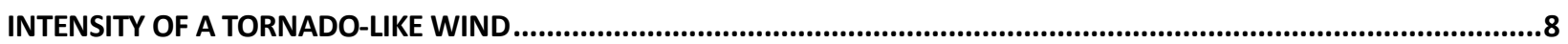

2.1 SUMMARY

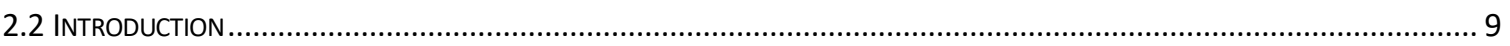

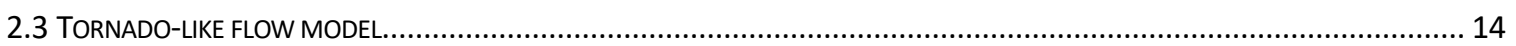

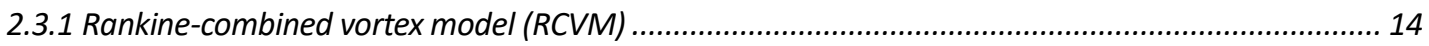

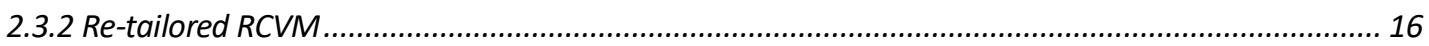

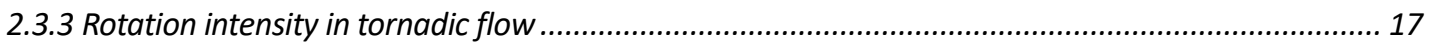

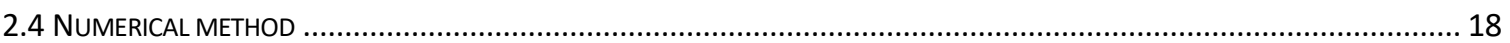

2.4.1 Lattice Boltzmann model with multiple-relaxation time collision .................................................... 18

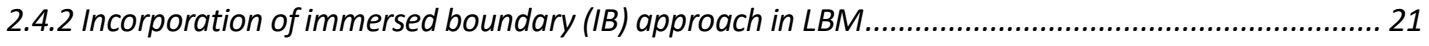

2.4.3 Smagorinsky subgrid-scale (SGS) model ............................................................................... 25

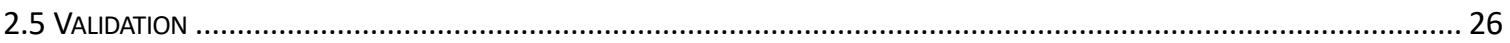

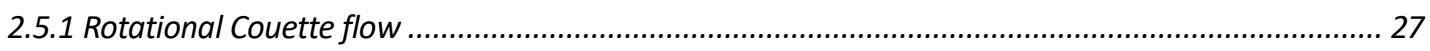

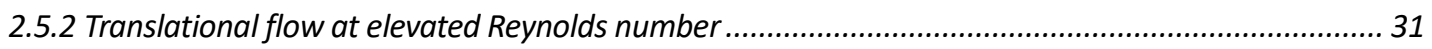

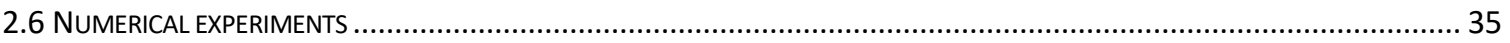

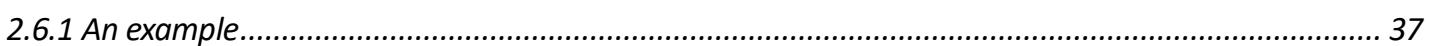

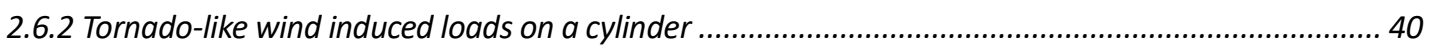




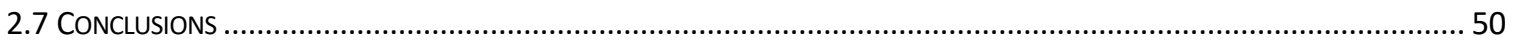

CHAPTER 3 A TWO-DIMENSIONAL IB-LBM FRAMEWORK COMBINED WITH RE-TAILORED RCVM FOR ASSESSING THE ROTATION INTENSITY OF A TORNADIC WIND OVER A BUILDING CONFIGURATION..................................................53

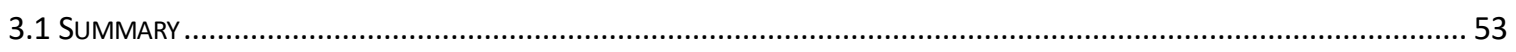

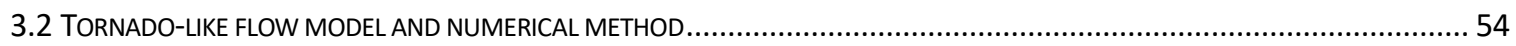

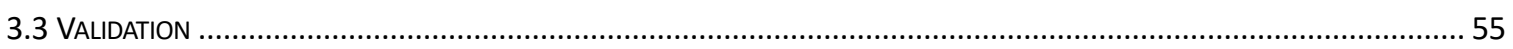

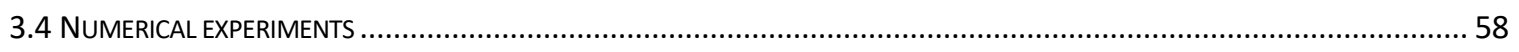

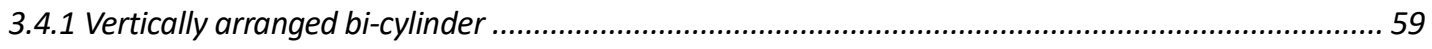

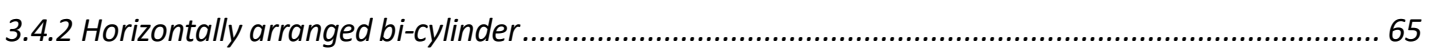

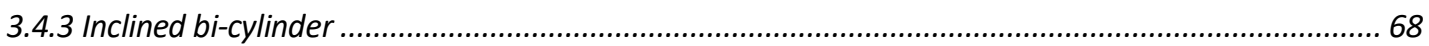

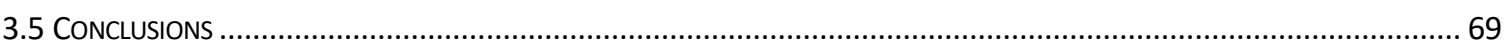

CHAPTER 4 AN IMMERSED-BOUNDARY (IB)-BASED TORNADO MODEL FOR COMPUTATIONAL ANALYSIS OF DISASTROUS WIND LOAD ON BUILDINGS - INVESTIGATION OF TORNADIC WIND LOAD OVER CYLINDER-QUINTUPLET73

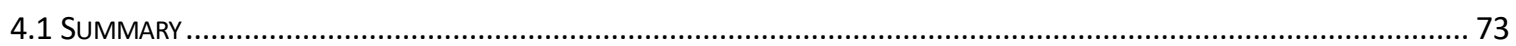

4.2 SETUP OF A CYLINDER-QUINTUPLET UNDER TORNADOES FROM DIFFERENT DIRECTIONS...............................................74

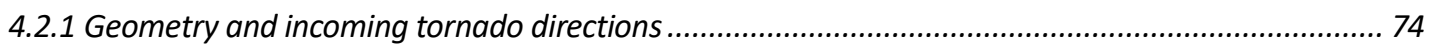

4.2.2 Dimensions of computational domain and mesh resolution ......................................................... 75

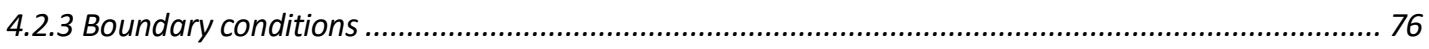

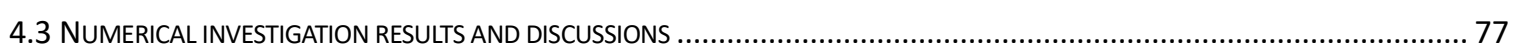

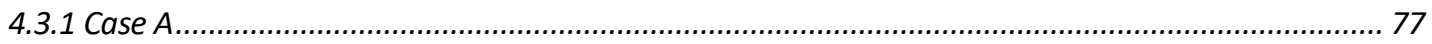

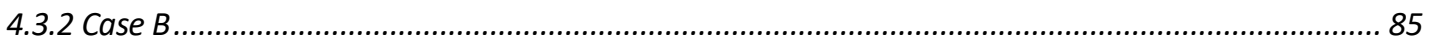

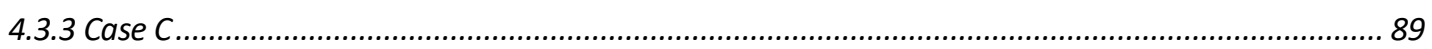

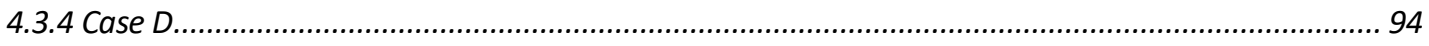

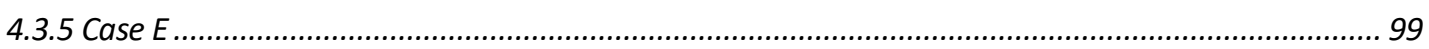

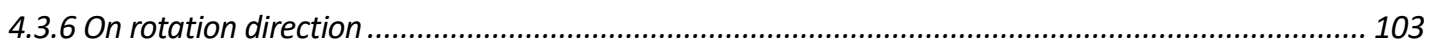

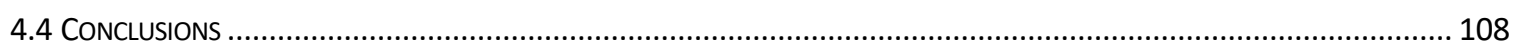

CHAPTER 5 A NOVEL THREE-DIMENSIONAL IMMERSED-BOUNDARY (IB) APPROACH BASED TORNADO MODEL POWERED BY PREDICTION-CORRECTION TECHNIQUE PART I - NUMERICAL METHODS AND BENCHMARK TESTING.111

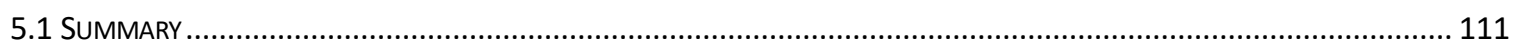

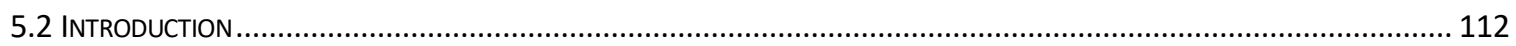

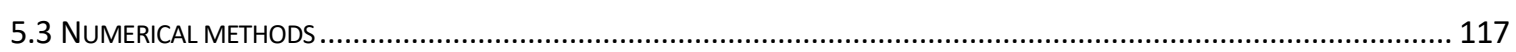

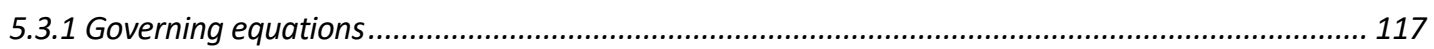

5.3.2 High-order finite difference discretization ............................................................................ 118

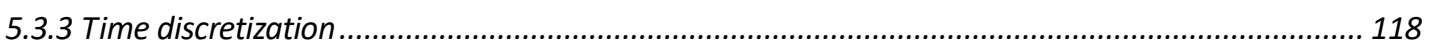




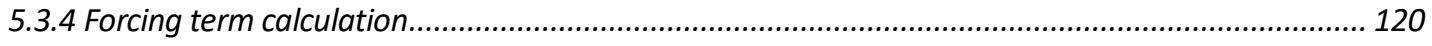

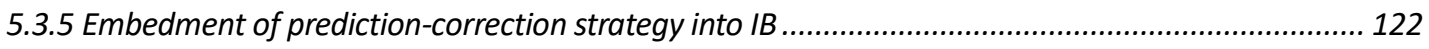

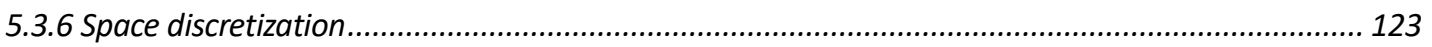

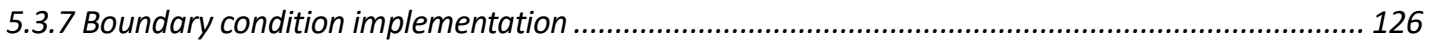

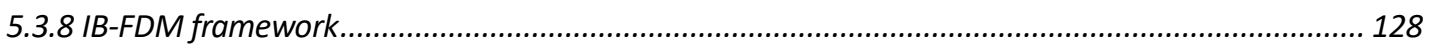

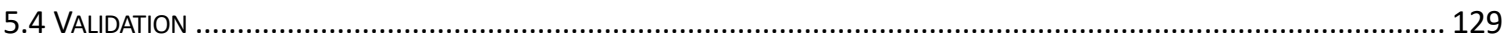

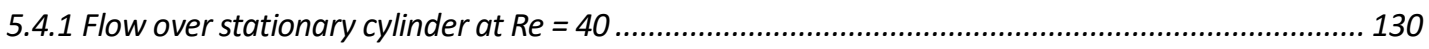

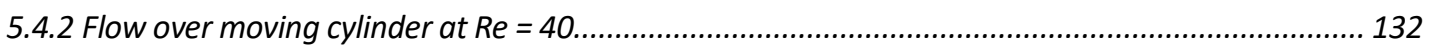

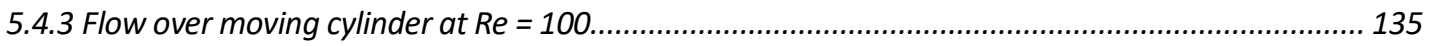

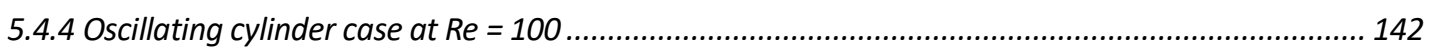

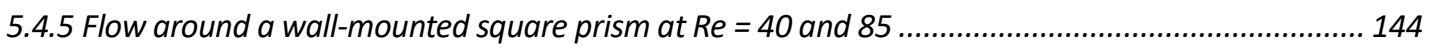

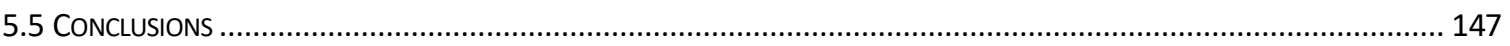

CHAPTER 6 A NOVEL THREE-DIMENSIONAL IMMERSED-BOUNDARY (IB) APPROACH BASED TORNADO MODEL POWERED BY PREDICTION-CORRECTION TECHNIQUE PART II - TORNADIC WIND SIMULATIONS ............................149

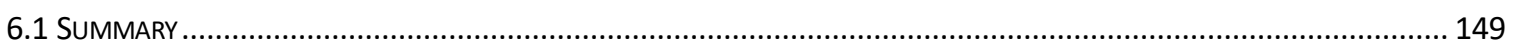

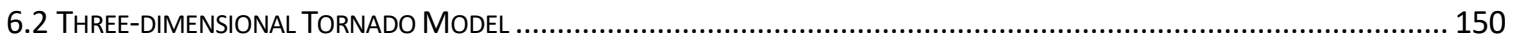

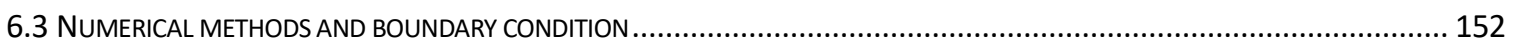

6.3.1 Computational geometry and initial parameters setup ........................................................... 152

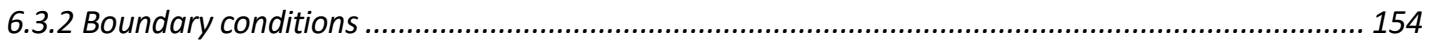

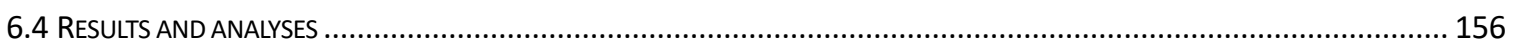

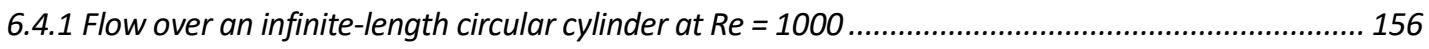

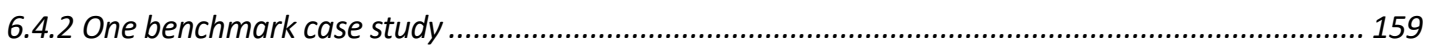

6.4.3 Quantitative analysis of loadings on different heights.............................................................. 168

6.4.4 Quantitative analysis between tornadoes over a prism and over a cylinder ............................... 173

6.4.5 Wind loadings comparison between models with and without prediction-correction technique ... 178

6.4.6 Comparison between 3D and 2D tornado-induced loadings .................................................... 180

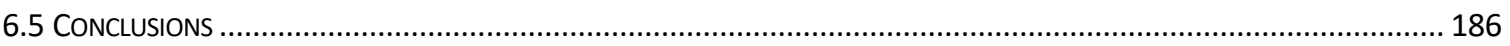

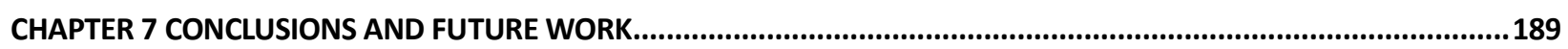

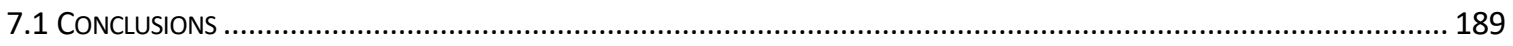

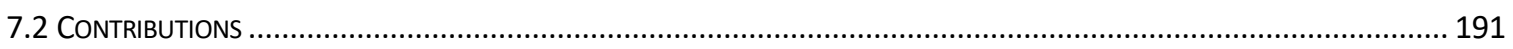

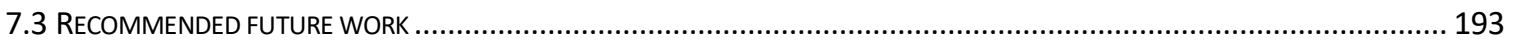

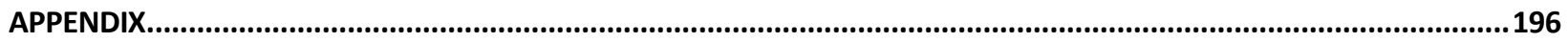

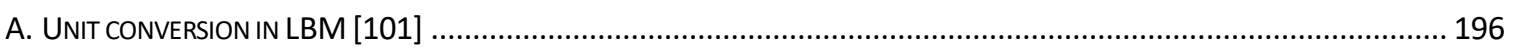

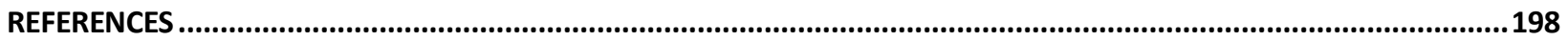

Viii 


\section{List of Tables}

Table 2.1 Comparisons of mean drag coefficient and Strouhal number at $R e=1000$.........................35

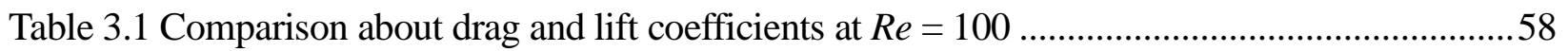

Table 3.2 Summarized results for three tested orientations ............................................................... 71

Table 5.1 Comparison of drag coefficient and length of recirculation zone at $\mathrm{Re}=40 \ldots \ldots \ldots \ldots \ldots \ldots \ldots . . . .132$

Table 5.2 Comparisons of time-averaged drag coefficient and Strouhal number at $\operatorname{Re}=100 \ldots \ldots \ldots .139$

Table 5.3 Tests for determining the numerical accuracy ................................................................. 142

Table 5.4 Comparison of drag and lift coefficients at $\mathrm{Re}=100$ for oscillating cylinder case............143

Table 5.5 Comparison of force coefficients at $\mathrm{Re}=40$ and 85 for flow over wall-mounted prism.. 147

Table 6.1 Mesh dependent check on flow over infinite cylinder at $\operatorname{Re}=1000 \ldots \ldots \ldots \ldots \ldots \ldots \ldots \ldots \ldots \ldots \ldots \ldots . . .157$

Table 6.2 Extremum comparison of force coefficients in different shapes .......................................177

Table 6.3 Extremum comparison of moment coefficients in different shapes ..................................177 


\section{List of Figures}

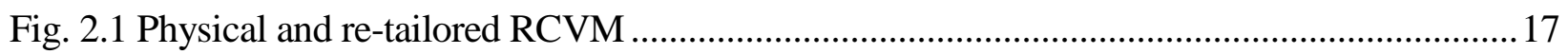

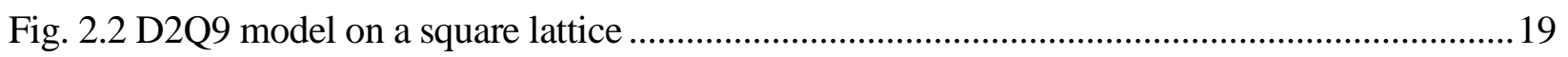

Fig. 2.3 Velocity magnitude contours (left: using ANSYS FLUENT; right: using LBM)..................30

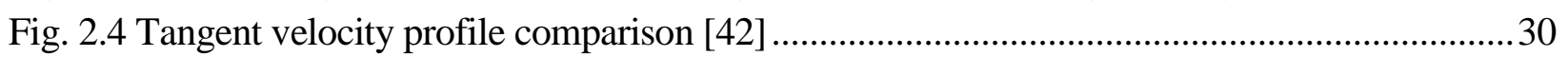

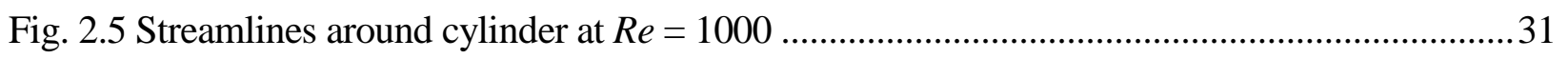

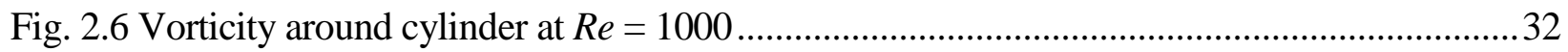

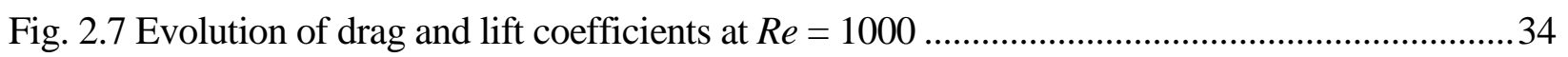

Fig. 2.8 Evolution of mean drag coefficient and comparison with references ....................................34

Fig. 2.9 Streamlines and velocity magnitude at "core-in" time............................................................39

Fig. 2.10 Streamlines and velocity magnitude at "coinciding-center" time..........................................39

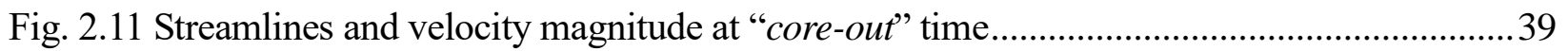

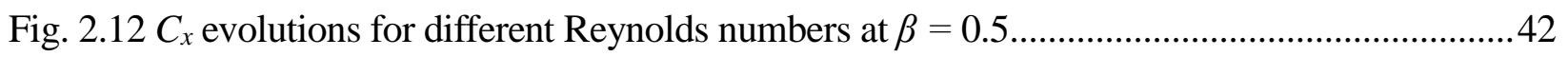

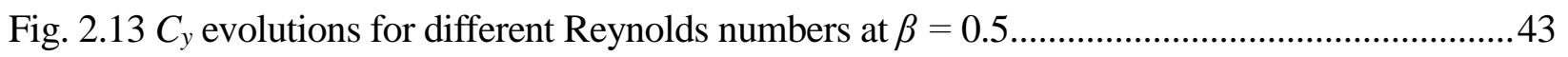

Fig. $2.14 C_{m}$ evolutions for different Reynolds numbers at $\beta=0.5$...............................................43

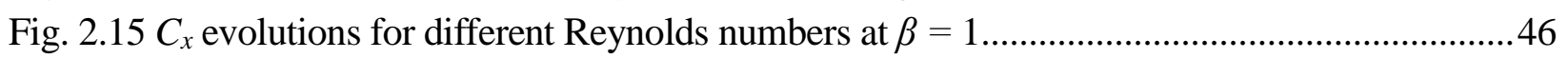

Fig. 2.16 $C_{y}$ evolutions for different Reynolds numbers at $\beta=1$...................................................4

Fig. $2.17 C_{m}$ evolutions for different Reynolds numbers at $\beta=1$.................................................4

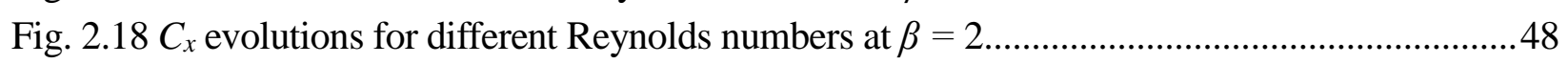

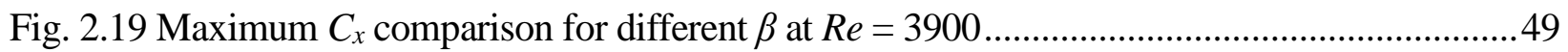

Fig. 3.1 Physical and re-tailored RCVM (a) physical tornado model; and (b) re-tailored tornado

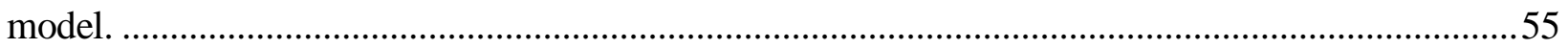

Fig. 3.2 Vorticity contour results from (a) the present method; (b) Wu et al. [51]; (c) Choi et al. [52]58 Fig. 3.3 Streamlines at "coinciding-center" time for vertically arranged bi-cylinder streamline........60 Fig. 3.4 $C_{x}$ evolutions for vertically arranged bi-cylinder at $\beta=1.5$, under different $R e$...................61

Fig. 3.5 $C_{y}$ evolutions for vertically arranged bi-cylinder at $\beta=1.5$, under different $R e$....................62

Fig. 3.6 $C_{m}$ evolutions for vertically arranged bi-cylinder at $\beta=1.5$, under different $R e$..................62

Fig. 3.7 $C_{x}$ evolutions for vertically arranged bi-cylinder at $\beta=2$, under different $R e$......................64 Fig. 3.8 $C_{x}$ extreme points (for the upper cylinder) for vertically arranged bi-cylinder at $\beta=2$, under different $R e$.

Fig. 3.9 Streamlines at "coinciding-center" time for horizontally arranged bi-cylinder streamline at $R e=1000$ and $\beta=1$. .66

Fig. 3.10 $C_{x}$ evolutions for horizontally arranged bi-cylinder at $\beta=1$, under different $R e$.................67

Fig. 3.11 $C_{y}$ evolutions for horizontally arranged bi-cylinder at $\beta=1$, under different $R e$.................67

Fig. 3.12 Streamlines at "coinciding-center" time for inclined bi-cylinder streamline at $R e=1000$ 
and $\beta=1.375$

Fig. 3.13 $C_{x}$ evolutions for inclined bi-cylinder at $\beta=1.375$, under different $R e$...........................69

Fig. 3.14 $C_{y}$ evolutions for inclined bi-cylinder at $\beta=1.375$, under different $R e$

Fig. 4.1 (a) Schematic for the cylinder-quintuplet study; (b) re-tailored cylinder-quintuplet scenario

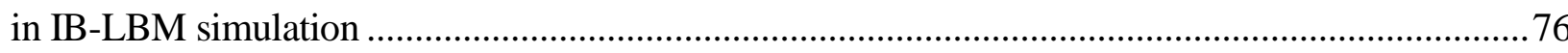

Fig. 4.2 Streamline contour for Case A at four representative time instants: (a).at right $r_{c}$; (b). tornado center; (c). at left $\mathrm{r}_{\mathrm{c}}$; (d). departed tornado)

Fig. 4.3 Velocity magnitude for Case A at four representative time instants: (a).at right $r_{c}$; (b).

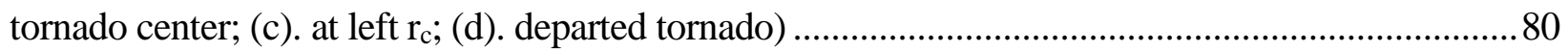

Fig. $4.4 \mathrm{X}$-direction force coefficient for Case A ......................................................................... 83

Fig. 4.5 Y-direction force coefficient for Case A ........................................................................... 83

Fig. 4.6 Moment coefficient for Case A .................................................................................... 83

Fig. 4.7 Three sets of testing cases for Case A with different mesh resolution and X-dimensional size .85

Fig. 4.8 Streamline contour for Case B at four representative time instants: (a).at right $r_{c}$; (b). tornado center; (c). at left $\mathrm{r}_{\mathrm{c}}$; (d). departed tornado).....

Fig. 4.9 Velocity magnitude for Case B at four representative time instants: (a).at right $r_{c}$; (b). tornado center; (c). at left $\mathrm{r}_{\mathrm{c}}$; (d). departed tornado) ................................................................... 87

Fig. 4.10 X-direction force coefficient for Case B ....................................................................... 88

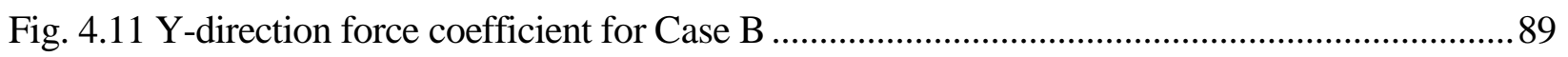

Fig. 4.12 Moment coefficient for Case B ...................................................................................... 89

Fig. 4.13 Streamline contour for Case C at four representative time instants: (a).at right $r_{c}$; (b). tornado center; (c). at left $\mathrm{r}_{\mathrm{c}}$; (d). departed tornado) .....................................................................91

Fig. 4.14 Velocity magnitude for Case $\mathrm{C}$ at four representative time instants: (a).at right $r_{c}$; (b). tornado center; (c). at left $r_{c} ;(d)$. departed tornado) ....................................................................92

Fig. 4.15 X-direction force coefficient for Case $\mathrm{C}$...................................................................... 93

Fig. 4.16 Y-direction force coefficient for Case C .......................................................................93

Fig. 4.17 Moment coefficient for Case C .................................................................................94

Fig. 4.18 Streamline contour for Case D at four representative time instants: (a).at right $r_{c}$; (b). tornado center; (c). at left $r_{c} ;(d)$. departed tornado) ...............................................................96 Fig. 4.19 Velocity magnitude for Case D at four representative time instants: (a).at right $r_{c}$; (b).

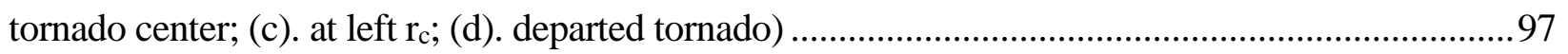

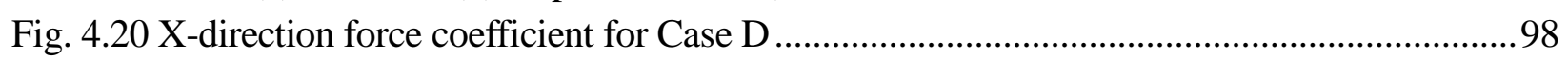

Fig. 4.21 Y-direction force coefficient for Case D......................................................................... 98

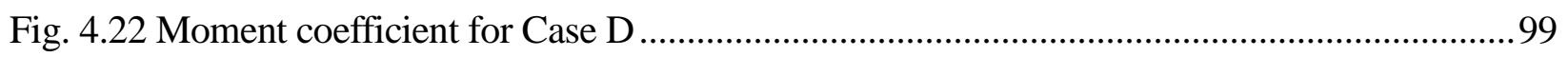

Fig. 4.23 Streamline contour for Case E at four representative time instants: (a).at right $r_{c}$; (b). tornado center; (c). at left $\mathrm{r}_{\mathrm{c}}$; (d). departed tornado) .100 
Fig. 4.24 Velocity magnitude for Case $E$ at four representative time instants: (a).at right $r_{c}$; (b). tornado center; (c). at left $\mathrm{r}_{\mathrm{c}}$; (d). departed tornado) ................................................................. 101 Fig. 4.25 X-direction force coefficient for Case E ..................................................................... 102

Fig. 4.26 Y-direction force coefficient for Case E ..................................................................... 102

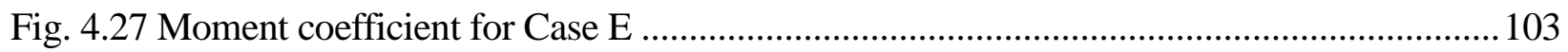

Fig. 4.28 Comparison between precedent cases and current case on different rotation direction..... 104

Fig. 4.29 Streamline contour for clockwise rotation of tornadic wind at four representative time instants: (a).at right $r_{c}$; (b). tornado center; (c). at left $r_{c}$; (d). departed tornado) ............................. 106

Fig. 4.30 Velocity magnitude for clockwise rotation of tornadic wind at four representative time instants: (a).at right $r_{c}$; (b). tornado center; (c). at left $r_{c}$; (d). departed tornado) ............................... 107

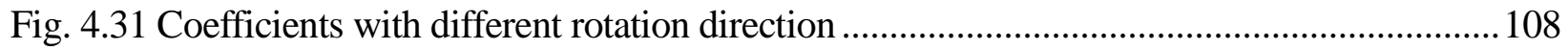

Fig. 5.1 Schematic of domain and boundary condition interpretation $\quad$............................128

Fig. 5.2 Distributions of streamline, flow over a stationary cylinder at $\mathrm{Re}=40 \ldots \ldots \ldots \ldots \ldots \ldots \ldots \ldots . . . . . . . . . . . .131$

Fig. 5.3 Zoom-in of streamline at the vicinity of cylinder.......................................................... 132

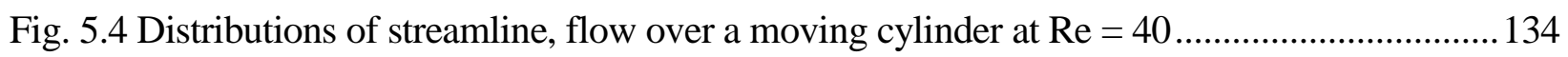

Fig. 5.5 Contour of velocity magnitude in three dimension at $\mathrm{Re}=40$..................................... 134

Fig. 5.6 Contour of velocity magnitude in one cross-section $(z=0.5)$ at $\operatorname{Re}=40 \ldots \ldots \ldots \ldots \ldots \ldots \ldots . . . . . .135$

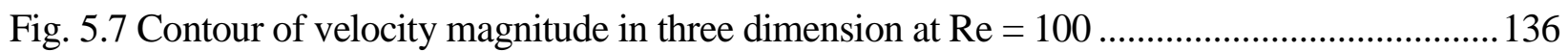

Fig. 5.8 Streamlines for flow over moving cylinder at $\mathrm{Re}=100$ (Two different time instants, $\mathrm{T} 1$ and

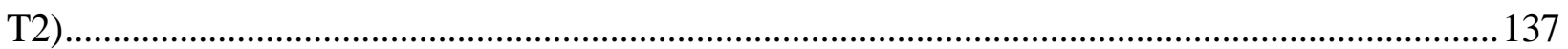

Fig. 5.9 Vorticity contours for flow over moving cylinder at $\mathrm{Re}=100$......................................... 138

Fig. 5.10 Comparison between original and prediction-correction scheme added results ............... 140

Fig. 5.11 Vorticity contour results from (a) present method; (b) Wu et al. [51]; (c) Choi et al. [52].144

Fig. 5.12 Schematic of computational domain ........................................................................ 145

Fig. 5.13 Mean velocity in z-direction and streamlines on the mid-transverse plane $(y=W / 2)$ at $\operatorname{Re}=$ 40 (top: present results; bottom: captured from [96]) ................................................................ 146

Fig. 6.1 Geometry and nomenclature of testing buildings: ........................................................ 154

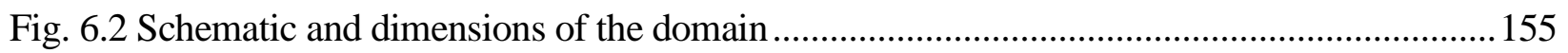

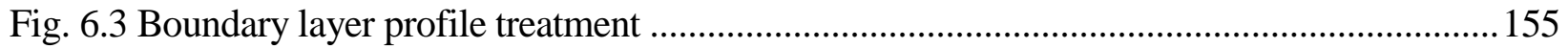

Fig. 6.4 Vorticity (vorticity $=1$ ) iso-surface from front view .................................................... 158

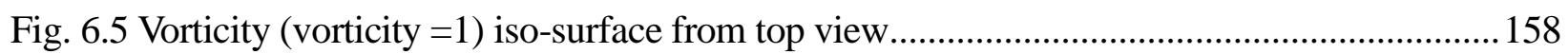

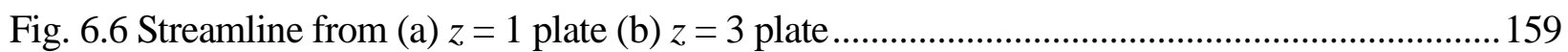

Fig. 6.7 Velocity magnitude in horizontal plane (top-view, $z=0.75)$. ......................................... 163

Fig. 6.8 Velocity magnitude in vertical plane (front-view, y=2): (a) Initial condition; (b) Pre-

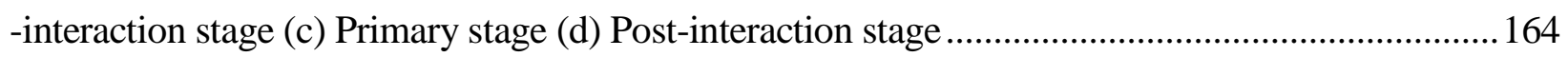

Fig. 6.9 Velocity magnitude contour: (a) Pre-interaction stage (b) Primary stage ........................... 165

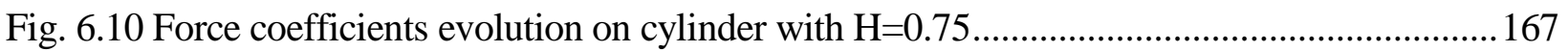


Fig. 6.11 Moment coefficients evolution on cylinder with $\mathrm{H}=0.75$ 167

Fig. 6.12 $\mathrm{C}_{\mathrm{x}}, \mathrm{C}_{\mathrm{y}}, \mathrm{C}_{\mathrm{z}}$ evolutions on cylinder for different heights.

Fig. $6.13 \mathrm{C}_{\mathrm{Mx}}, \mathrm{C}_{\mathrm{My}}, \mathrm{C}_{\mathrm{Mz}}$ evolutions on cylinder for different heights 170

Fig. 6.14 $\mathrm{C}_{\mathrm{x}}, \mathrm{C}_{\mathrm{y}}, \mathrm{C}_{\mathrm{z}}$ evolutions on prism for different heights. 172

Fig. 6.15 $\mathrm{C}_{\mathrm{Mx}}, \mathrm{C}_{\mathrm{My}}, \mathrm{C}_{\mathrm{Mz}}$ evolutions on prism for different heights 173

Fig. 6.16 Force coefficient evolutions for different shape at $\mathrm{H}=0.5$ 175

Fig. 6.17 Moment coefficient evolutions for different shape at $\mathrm{H}=0.5$ 175

Fig. 6.18 Force (a) and Moment (b) coefficient evolutions for different shape at $\mathrm{H}=1.0$........... .176

Fig. 6.19 Force (a) and Moment (b) coefficient evolutions for different shape at $\mathrm{H}=1.75$ 177

Fig. 6.20 Cx evolutions on cylinder for different heights, without prediction-correction technique 180 Fig. 6.21 Force coefficients evolution over 2D cylinder scenario.

Fig. $6.22 \mathrm{C}_{\mathrm{x}}$ evolution for each portion over $3 \mathrm{D}$ cylinder scenario: (a). all portions; (b). zoom in to $3 / 10$ to $6 / 10$ portions 183

Fig. $6.23 \mathrm{C}_{\mathrm{y}}$ evolution for each portion over 3D cylinder scenario: (a). all portions; (b). 184 Fig. 6.24 $\mathrm{C}_{\mathrm{Mz}}$ evolution for each portion over 3D cylinder scenario: (a). all portions; (b). zoom in to $3 / 10$ to $6 / 10$ portions ... .184

Fig. 6.25 Force coefficients evolution over 2D prism scenario 185 Fig. 6.26 $\mathrm{C}_{\mathrm{x}}$ evolution for each portion over 3D prism scenario: (a). all portions; (b). zoom in to 3/10 to $6 / 10$ portions .

Fig. 6.27 $\mathrm{C}_{\mathrm{y}}$ evolution for each portion over 3D prism scenario: (a). all portions; (b). zoom in to 3/10 to $6 / 10$ portions 186

Fig. 6.28 $\mathrm{C}_{\mathrm{Mz}}$ evolution for each portion over 3D prism scenario: (a). all portions; (b). zoom in to $3 / 10$ to $6 / 10$ portions) 186 


\section{Nomenclature}

\section{English Symbols}

\begin{tabular}{|c|c|}
\hline$A$ & Normalized rotation amplitude \\
\hline$c_{i}$ & discrete velocities \\
\hline$C_{d}$ & Drag coefficient \\
\hline$C_{l}$ & Lift coefficient \\
\hline$C_{x}$ & Force coefficient in $\mathrm{x}$-direction \\
\hline$C_{y}$ & Force coefficient in y-direction \\
\hline$C_{z}$ & Force coefficient in z-direction \\
\hline$C_{m}$ & Moment coefficient in 2D simulation \\
\hline$C_{M_{x}}$ & Moment coefficient in $\mathrm{x}$-direction \\
\hline$C_{M_{y}}$ & Moment coefficient in y-direction \\
\hline$C_{M_{z}}$ & Moment coefficient in z-direction \\
\hline$C_{s}$ & Smagorinsky constant \\
\hline$c_{s}$ & Lattice speed \\
\hline$c$ & Dimensionless ratio of grid and time step \\
\hline$d$ & Width of building in 3D \\
\hline$D$ & Diameter of cylinder \\
\hline
\end{tabular}




\begin{tabular}{|c|c|}
\hline$D_{i j}$ & Dirac interpolation function \\
\hline$\vec{e}$ & Particle velocities \\
\hline$\vec{F}$ & Boundary force density \\
\hline$F_{D}$ & Drag component of the aerodynamic force \\
\hline$F_{L}$ & Lift force of the aerodynamic force \\
\hline$F_{x}$ & Aerodynamic force in $\mathrm{x}$-direction \\
\hline$F_{y}$ & Aerodynamic force in $y$-direction \\
\hline$F_{Z}$ & Aerodynamic force in z-direction \\
\hline$F_{R S T}$ & Restoring force at Lagrangian point \\
\hline$f_{R S T}$ & Restoring force at fluid point \\
\hline$\vec{f}$ & Force density acting on the fluid \\
\hline$f$ & Particle distribution function \\
\hline$f_{q}$ & Vortex shedding frequency \\
\hline$f$ & Space of distribution functions used in MRT \\
\hline$H$ & Height of the domain in 3D \\
\hline$h$ & Height of building in 3D \\
\hline$j$ & Mass flux used in MRT \\
\hline $\mathrm{L}$ & Length of recirculation zone \\
\hline
\end{tabular}




\begin{tabular}{|c|c|}
\hline$m$ & Moment space of distribution functions used in MRT \\
\hline $\mathbf{M}$ & Transformation matrix used in MRT \\
\hline M & Resulting moment in 2D simulation \\
\hline $\mathrm{M}_{\mathrm{x}}$ & Resulting moment in $\mathrm{x}$-direction \\
\hline $\mathrm{M}_{\mathrm{y}}$ & Resulting moment in y-direction \\
\hline $\mathrm{M}_{\mathrm{z}}$ & Resulting moment in z-direction \\
\hline$p$ & Pressure \\
\hline$q$ & Energy flux \\
\hline$\vec{Q}_{i j}$ & Momentum flux tensor \\
\hline $\operatorname{Re}$ & Reynolds number \\
\hline$r$ & Distance from tornado center \\
\hline$r_{c}$ & Critical radius of tornado \\
\hline $\mathbf{S}$ & Relaxation matrix \\
\hline$S_{x}$ & Projection area in $\mathrm{x}$-direction \\
\hline$S_{y}$ & Projection area in y-direction \\
\hline$S_{z}$ & Projection area in z-direction \\
\hline$\vec{S}_{i j}$ & Strain rate tensor \\
\hline$s$ & Lagrangian parametric coordinates \\
\hline
\end{tabular}




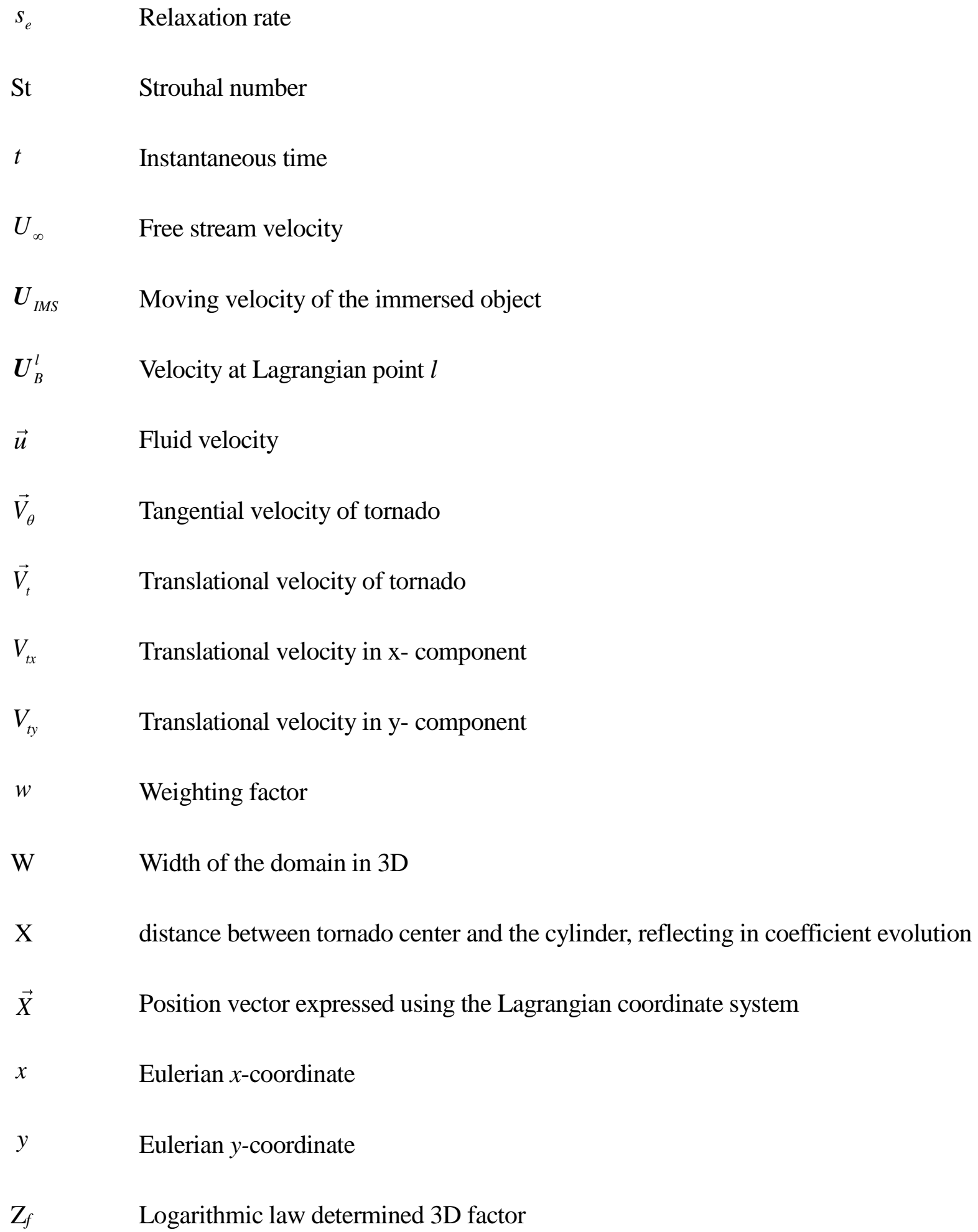


\# $\quad$ Number (No.)

\section{Greek Symbols}

\begin{tabular}{|c|c|}
\hline$\alpha_{1}, \alpha_{2}$ & Negative free constant used in feedback forcing method \\
\hline$\beta$ & Rotation intensity of tornado \\
\hline$v$ & Kinematic viscosity \\
\hline$\Gamma$ & Boundary curve \\
\hline$\Omega$ & Flow field \\
\hline$\tau$ & Relaxation time \\
\hline$\delta$ & Dirac delta function \\
\hline$\rho$ & Density \\
\hline$\zeta$ & Bulk viscosity \\
\hline$\omega$ & Angular velocity \\
\hline$\Delta x$ & Grid space in the $x$-direction \\
\hline$\Delta y$ & Grid space in the $y$-direction \\
\hline$\Delta_{L}$ & Filter width \\
\hline$\delta$ & Delta function \\
\hline$\delta x$ & Lattice size \\
\hline
\end{tabular}




\section{Subscripts/Superscripts}

$\begin{array}{ll}()^{*}()^{* *} & \text { Intermediate variable } \\ ()_{a} & \text { Number of directions of velocities at each node } \\ ()_{B}^{l} & l^{\text {th }} \text { Lagrangian point } \\ ()_{i}^{j} & j^{\text {th }} \text { time step for a Lagragian point } i \\ ()_{\text {boundary }} & \text { Boundary point } \\ ()_{\text {fluid }} & \text { Fluid point } \\ ()_{\text {object }} & \text { Object in Fluid } \\ ()_{\infty} & \text { Far field } \\ ()_{x} & x \text {-direction } \\ ()_{y} & y \text {-direction } \\ ()^{e q} & \text { Equilibrium state }\end{array}$




\section{Chapter 1 Introduction}

\subsection{Background and motivation}

Tornadoes are more frequently reported in Canada than any other country except the United States. On average, there are nearly 100 confirmed and unconfirmed tornadoes annually that touch down in Canada, with most occurring in Southern Ontario, the southern Canadian Prairies and southern Quebec [1]. The most common types are EF0 to EF2 (less than $135 \mathrm{mph}$ ) in damage intensity level, and fewer than 5\% of tornadoes in Canada are rated EF3 or higher in intensity, where the wind speed excesses $135 \mathrm{mph}$. Rotating and moving rapidly within a brief time period, a tornado may generate a highly turbulent wind field over ground, which may inflict colossal structural damage, such as uprooted trees, toppled power lines, demolished houses, and even take numerous human lives.

Tornadoes are well-known as one of the most difficult subjects in the field of wind engineering, due to their violent and obscure nature. Therefore, a keen understanding of the underlying mechanism of tornado, as well as its interaction with building constructions, can significantly mitigate the property damages induced by the tornadic wind. Furthermore, a better knowledge of tornado-induced loadings on building constructions can lead to more accurate prediction of maximum forces, which is considered as imperative for building design. 
A variety of approaches aimed at collecting data from tornadoes, such as satellite and radar imaging [2]-[4] as well as laboratory simulation [5]-[13], have been employed by the tornado research community. Since the study of tornado dynamics through field observations and laboratory experiments tends to be time-consuming, restrictive, and expensive, more research efforts in mathematical modeling and numerical simulation have been made to gain further understanding of the intricate characteristics of tornadic wind field.

\subsection{Up-to-date progress}

From the first remarkable experiment conducted by Ward [5] tracing back to 1972, there had been a lot of laboratory simulators developed by the wind engineering community [5]-[13]. For instance, the ISU (Iowa State University) tornado-like vortex simulator [9]-[10] is designed and constructed to be able to generate a tornado-shape vortex that can translate along the ground plane to interact with structure models on the ground. The simulator also has the ability to accommodate models of reasonable size for measuring loads on structures and buildings scaling from 1:100 for low-rise buildings and 1:500 for high-rise buildings and large structures. Another successful tornado simulator is named as the Wind Engineering, Energy and Environment (WindEEE) Dome [11]-[13],

developed by the Western University, which employed the fans on each of the walls to push air in through a set of directional louvers creating various of tornado-like vortices. Other type of wind system, such as downburst, sheared winds can also be simulated in the WindEEE Dome [11]. 
Numerically, over the past few decades, Wilson [14] first applied a two-dimensional tornado model to examine the effects of tornadoes on rectangular-shaped buildings. Nolan and Farrell [15] used the axisymmetric Navier-Stokes (N-S) equations with a constant viscosity to explore the structure and dynamics of tornado-like vortices. Lewellen et al. [16]-[17] employed a three-dimensional large eddy simulation (LES) turbulence model to investigate the dynamics of a tornado-like vortex near the surface with particular attention paid to the turbulent flow characteristics in the corner region. Ishihara et al. [18] developed a tornado model by incorporating LES in the time-dependent N-S equations to simulate the flow fields of one-celled and two-celled vortices. On the other hand, the numerical simulations conducted by Selvam et al. placed their focus on the tornado-building interaction based on the Rankine Combined Vortex Model (RCVM), which is of more practical significance. They reported the tornado-induced loads on 2D sections of a cylinder and multi-cubic buildings using the finite difference discretization of the N-S equations [19]-[20], and also extended their simulations to 3D cases [21] together with the case of tornadoes at elevated Reynolds numbers with the aid of a LES model [22]. Their preliminary simulation results suggested that the important tornado-building interaction parameters mainly include the tornado translation velocity, core size, maximum tangential speed, and the building shape. Kuai et al.[23] embedded k- $\varepsilon$ model to commercial software FLUENT to study the parameter sensitivity for the flow fields of a laboratory-simulated tornado based on ISU tornado-like vortex simulator. Cao et al. [24] used time-dependent unsteady finite volume method to simulate the wind pressure and loadings 
on low-rise building induced by tornado, which was also carried out with respect to ISU-type simulator.

However, the majority of previous numerical simulations have their drawbacks. The effect of translation motion was often neglected when numerical tornado models were built based on Ward-type or ISU-type simulator [14], [15], [18], [24] due to the limitation of the model. On the contrary, when taking translating effect into consideration, the boundary condition of the domain governed by tornado is highly time-dependent [19]-[21], so that the boundary condition should be updated every time step during the simulation, which is very tedious.

\subsection{Thesis objectives}

Due to the aforementioned restrictions in the computational tornado dynamics study, the literature on numerical investigation of tornado-induced loadings on ground buildings was still very limited. The present work is aiming to numerically open a novel outlet for simulation of tornado-building interaction, which will not only take all major tornado characteristics into consideration, but also select more ingenious CFD tools to overcome the computational obstacles. The main objectives of this thesis are as follows:

1) Develop novel computational frameworks, both in two-dimension (2D) and three-dimension (3D), to simulate tornado-construction interaction using hybrid numerical approaches;

2) Examine the tornado-induced loadings (force and moment in the $3 \mathrm{D}$ environment) on 
various building groups, including single cylinder, bi-cylinders, and cylinder-quintuplet structures, and explore the tornado loading change mechanism in response to the variation of tornado translation direction.

3) Investigate the potential influence factors that affect the loadings, such as Reynolds number, rotational intensity, building height, building shape, etc.;

4) Provide practical guidelines for enhancement in the design of building constructions towards better wind-resistant capabilities.

\subsection{Thesis structure and contributions}

This is a manuscript-style thesis, which is based upon the following published papers or completed manuscripts that have been submitted or will be submitted very shortly:

(1) Xixiong Guo, Jun Cao. An IB-LBM investigation into the aerodynamic coefficients in relation to the rotation intensity of a tornado-like wind. Computers \& Mathematics with Applications, 78(4), 1206-1226, 2019. (Chapter 2)

(2) Xixiong Guo, Rangaraj Palanisamy, Jun Cao. A two-dimensional IB-LBM framework combined with re-tailored RCVM for assessing the rotation intensity of a tornadic wind over a building configuration. Engineering Structures, 131, 57-68, 2017. (Chapters 3)

(3) Xixiong Guo, Jun Cao. An Immersed-Boundary (IB)-Based Tornado Model for Computational Analysis of Disastrous Wind Load on Buildings - Investigation of Tornadic Wind Load over 
Cylinder-Quintuplet (Submitted to Journal of Wind Engineering \& Industrial Aerodynamics).

\section{(Chapter 4)}

(4) Xixiong Guo, Jun Cao. A Novel Three-Dimensional Immersed-Boundary (IB) Approach Based Tornado Model Powered by Prediction-Correction Technique Part I - Numerical Methods and Benchmark Testing (ready to submit to Journal of Computational Physics). (Chapter 5)

(5) Xixiong Guo, Jun Cao. A Novel Three-Dimensional Immersed-Boundary (IB) Approach Based Tornado Model Powered by Prediction-Correction Technique Part II - Tornado wind simulations (ready to submit to Journal of Computational Physics). (Chapter 6)

Chapters 2 - 4 focus on establishing a preliminary two-dimensional model to simulate tornado-building interaction. Inspired by the success of immersed-boundary (IB) method in dealing with moving/complex boundary problems, as well as its conjunction with lattice Boltzmann method (LBM) for a great number of fluid flow problems, a novel IB-LBM framework is firstly proposed for investigation of tornadic wind effects in two dimensional (2D) scenario. With the aid of IB method, the highly time-dependent boundary condition, which is considered as the biggest challenge in tornado simulation, is turned to a manageable time-invariant condition. Furthermore, the wind loadings in relation to the rotation intensity of tornado on various building constructions are also assessed. Both translational and rotational components of tornadoes are found to have significant impact on the wind loadings.

Then, a three-dimensional tornado model is developed in Chapters 5 - 6 , after taking the vertical 
velocity component into consideration. A highly parallel computational framework based on an open-source toolkit named Incompact3d is developed with a seamless embedment of the feedback forcing based IB model and prediction-correction algorithm into the code. The new framework is applied to investigate a 3D tornado field that includes buildings with different shapes as objects sustaining wind loadings. Comprehensive interpretations, especially the uprooting effect in vertical direction, are provided.

Except the collaboration with Rangaraj Palanisamy, who was a master student supervised by Prof. Jun Cao in 2015-2017, which was reflected in the data collection and visualization of tests as shown in Section 3.3, I declare that all the rest work was completed by myself under the supervision of Prof. Jun Cao. 


\section{Chapter 2 An IB-LBM investigation into the aerodynamic coefficients in relation to the rotation intensity of a tornado-like wind}

This chapter is based on the following published paper:

Xixiong Guo, Jun Cao. An IB-LBM investigation into the aerodynamic coefficients in relation to the rotation intensity of a tornado-like wind. Computers \& Mathematics with Applications, 78(4), 1206-1226, 2019.

\subsection{Summary}

A tornadic wind is essentially considered as an airflow that simultaneously translates and rotates. Numerical simulations of this kind of hybrid flow remain inadequate due to many numerical difficulties, one of the major challenges consisting in the establishment of a set of boundary conditions that are, for the tornado-obstacle interaction scenario, both rational in physics and simple in numerical implementation. Inspired by the success of immersed-boundary (IB) lattice Boltzmann method (LBM) for simulations of fluid-structure interaction problems, this study proposes a new outlet of the IB-LBM framework for investigation of tornadic wind effects, featuring a reformed interpretation of the Rankine-Combined Vortex Model (RCVM) that considerably facilitates the boundary condition setup. Moreover, the main purpose of this chapter is to examine the tornadic wind loading in relation to the rotation intensity of a tornado, and presents a practical Newton's 
bi-section-like method for determining the critical rotation intensity beyond which the aerodynamic coefficients no longer increase when Reynolds number rises. This critical rotation intensity serves to characterize tornadic winds, such that the tornado with a rotation density below its critical value can be considered as mainly dominated by the translation part and, otherwise, the dominance no longer belongs only to the translational component of the tornado. Since it has been rather conventional that, when studying tornadoes, Reynolds number is determined using only the translation velocity as characteristic velocity, the present tornado study intends to suggest, through a number of numerical test series, that more attention be paid to the insufficiently explored rotational component, which physically tends to play a more dominant role when an intensive rotation is present in a tornado scenario.

\subsection{Introduction}

As meteorological disaster, a tornado may induce a significantly elevated level of wind forces if compared to a straight-line wind, and cause severe property damages. The tornado dynamics has been experimentally studied by many tornado research communities [5]-[11]. Due to the obvious drawbacks in using laboratorial simulator, including long set-up time, considerable expense, and other restrictive conditions, more efforts are nowadays made by the tornado research community in mathematical modeling and numerical simulation of the tornado phenomena. Obviously, a properly designed model for a straight-line wind would fail to describe a tornado-like wind even if the two 
winds have the same translational speed [25]. This is because a tornado can be essentially decomposed into simultaneous translational and rotational flow components, and both have to be considered in the model for a tornado. A well-established tornado model has always been sought, since it would help the researchers better understand, through economical computer-aided simulations, the tornado dynamics as well as the mechanism of tornado-building interaction and, ultimately, improve the design of buildings towards their enhanced wind-resistant capabilities.

Over the past few decades, tornado-induced wind loads exerted on constructions have been also a practical numerical simulation topic. However, very little literature can be found that numerically investigated the tornadic wind loadings on buildings. Two representative pieces of work in this regard are inspirational to the present study. The numerical simulations performed by Selvam et al. [19]-[21] employed the Rankine-combined Vortex Model (RCVM) with focus placed on the tornado-building interaction. They reported the tornado-induced loads on 2D sections of a cylinder and multi-cubic buildings using the finite difference discretization of the N-S equations [19]-[20], with further extensions to 3D cases [21] at elevated Reynolds numbers. Also, Bienkiewicz and Dudhia [26] conducted comparative studies to examine the wind loads and surface pressure on small building models in the cases of swirling, tornado-like, and straight-line winds, finding that the loadings induced by the swirling and tornado-like winds are significantly higher (3-5 times) than in the case of a straight-line wind, and the surface pressure distributions are also quite different between each other. 
An important remark made by [26] for the tornado research community is that the effect of a tornadic wind is of strongly hybrid nature since both translational and rotational airflow components take part in a tornado scenario, thus, reliance on the translation part of a tornado for establishment of tornado research framework, such as employing the translation velocity component for determining the Reynolds number of a tornado and then using such a translational speed based Reynolds number to characterize tornadoes, tends to make little sense once the rotation part of a tornado is intensive enough to overcome the dominance of the translational component. However, further investigation into the influence of the rotational part to the overall tornado wind loading effects is so far found very rare in existing literature. Without sufficient examination about the impact of the rotational component in a tornado, the understanding of the tornado dynamics and the tornado-building interaction mechanism may remain incomplete or even inaccurate, and skewed perspective might result from the tornado framework built excessively upon the translational part but defectively upon the rotational part. In order to acquire a better comprehension about the tornadic wind induced loads on a civil construction, this study aims to place a particular probe, through numerical simulations with the aid of RCVM-based tornado model, into the relation between the aerodynamic coefficients and the rotational component of a tornadic wind.

The existing tornado simulations [16]-[21] mainly relied on conventional numerical methods to build their N-S based solvers. When solving the N-S equations, the obviously time-variant boundary conditions of the tornado-like flow field have to be updated for every time step. Different from the 
reported tornado solvers, the present study propose a novel lattice Boltzmann method (LBM) based computational framework with the immersed boundary (IB) approach properly embedded, such that the tedious updating process for prescribing the boundary conditions of tornado simulation domain can be successfully circumvented.

In fact, as an alternative to the Navier-Stokes equations based solver, LBM [27] has been often used for simulating viscous flow in recent years due to its noticeable advantages in terms of simplicity for implementation, parallelizability for algorithmic development, and robustness for applications [27]-[29]. On the other hand, the IB method proposed by Peskin [30]-[31] is an attractive approach when a moving boundary problem is dealt with. As a non-boundary conforming approach, IB introduces an additional "restoring" force in the vicinity of an moving object that is immersed in a fluid, so that all the effects of the fluid to the boundary of the solid object can be taken into account and, then, this additional force is distributed back to the affected grid nodes to form a special body force. In 2004, the IB scheme was successfully incorporated in LBM by Feng and Michaelides [32]. They demonstrated the remarkable advantage of IB-LBM when simulating 2D and 3D particulate flows [33]. Lately, considerable IB-LBM progress has been achieved in solving challenging fluid-solid interaction (FSI) problems, such as biomimetic simulation focusing on flapping/flexible foil [34]-[35] and the flapping of multiple elastic structures [36], particulate-fluid interaction flow [37], solid-liquid phase change problems [38].

Inspired by the reported IB-LBM application success [32]-[38] this study aims to numerically 
open a novel IB-LBM outlet for simulation of tornado-building interaction. As the tornado-like wind can be decomposed into two components, namely, rotation and translation, the scenario of a tornado towards a building is re-interpreted, in this study, as a "virtual" translation of the building towards a rotating airflow about its "virtually locked" center, which implies the application of the "relative motion" principle to the translation component of the tornadic wind; then, the IB approach is employed to mathematically model the "virtually moving" building. This novel interpretation for the tornado-building interaction greatly facilitates the boundary condition set up. That is, the outer boundary of the tornadic wind domain can be prescribed by simply using the rotation part of the tornado according to RCVM, while the satisfaction of the no-slip boundary condition for the building can be guaranteed by the IB model. Guided by this novel interpretation, under numerical investigation are a number of test series for tornado-like flows with different rotation intensities that pass over an obstacle, and a detailed study will be conducted that focuses on the impact of the rotation intensity to the overall tornado-induced forces.

The rest of this chapter will unfold as follows. The tornadic wind model, referred to as Rankine-combined Vortex Model (RCVM), along with its re-tailored version is presented in Section 2.3. Section 2.4 describes the IB-LBM framework that is employed in this tornado simulation study, followed by Section 2.5 in which the present IB-LBM based tornado model is validated using separated translational and rotational flow cases. In Section 2.6, a number of test series corresponding to tornadic flows over a cylinder are examined with focus particularly placed on the 
effects of the rotation intensity to the overall wind loadings exerted on the obstacle. Finally, concluding remarks are made in Section 2.7, emphasizing the necessity of further exploring the rotational component of tornado in order to gain a more complete comprehension about the tornado dynamics as well as, for more practical reason, the tornado-building interaction mechanism.

\subsection{Tornado-like flow model}

\subsubsection{Rankine-combined vortex model (RCVM)}

The Rankine-combined vortex model (RCVM) is the simplest model to describe a tornado flow domain. The RCVM featuring inherently satisfying the Navier-Stokes equation [20] while exhibiting a simple analytic form is pertinent for describing a tornado-like wind over a two-dimensional obstacle-free domain. The RCVM velocity profile can be decomposed into a constant-translation component, $\vec{V}_{t}$, and a component of rotation with respect to the wind center, $\vec{V}_{\theta}$, which denotes the tangential velocity within the circular-motion context. According to RCVM, $V_{\theta}$ varies with the distance from the vortex center, $r$. Let $r_{c}$ represent the critical radius where the peak tangential velocity, $\left(V_{\theta}\right)_{\max }$, takes place. When $r \leq r_{c}, V_{\theta}=r \omega$ indicates the tangential velocity increases linearly with $r$ at a constant angular velocity $\omega$; when $r>r_{c}, V_{\theta}=$ $r_{c}^{2} \omega / r$ shows the tangential velocity decreases right after the presence of $\left(V_{\theta}\right)_{\max }$. The expressions can be summarized as: 


$$
V_{\theta}= \begin{cases}r \omega, & r \leq r_{c} \\ r_{c}^{2} \omega / r, & r>r_{c}\end{cases}
$$

When an obstacle is present in a tornadic wind field, the idealized RCVM is no longer applicable to the entire flow domain due to the wind-obstacle interaction. In practice, RCVM still can serve to initialize the flow domain, physically representing a tornadic wind that has not been affected by the obstacle provided the wind center and the obstacle are distant from each other at the beginning. Remark that real tornado scenario unfolds with an identical translational velocity, $\vec{V}_{t}=V_{t x} \vec{i}+V_{t y} \vec{j}$, applicable to the whole flow domain while the building remains immobile, in addition to the rotation about the moving wind center. In the light of RCVM, at a given time instant, $t$, the resultant velocity at a point $(x, y)$ located in the counterclockwisely-rotating tornado field reads:

$$
\vec{V}=\vec{V}_{t}+\vec{V}_{\theta}=\left(V_{t x}-V_{\theta} \sin \theta\right) \vec{i}+\left(V_{t y}+V_{\theta} \cos \theta\right) \vec{j}
$$

with

$$
\theta=\tan ^{-1}\left(\frac{y-y_{c}(t)}{x-x_{c}(t)}\right)
$$

where $x_{c}(t)=x_{c 0}+v_{t x} t$ and $y_{c}(t)=y_{c 0}+v_{t y} t$, and $\theta$ stands for the angle between the radial line passing through $(x, y)$ and the horizontal direction pointing to the right. Clearly, in RCVM, $\theta=\theta(x, y, t)$ and, in turn, $\vec{V}=\vec{V}(x, y, t)$. 


\subsubsection{Re-tailored RCVM}

Due to the translation of the tornado center, the resultant velocity in RCVM eventually turns out to be $\vec{V}=\vec{V}(x, y, t)$. In this sense, if attempting to employ RCVM for prescription of the boundary condition, updating the boundary condition becomes mandatory due to its time-dependent nature, which poses computational complexity if purely relying on conventional Computational Fluid Dynamics (CFD) methods without any modification.

This tornado modeling study intends to re-tailor the original RCVM such that the boundary condition of a tornadic wind domain with presence of an obstacle can be established still on the basis of RCVM but in a time-independent form. That is, by using the concept of "relative motion", the translation part of RCVM is detached from the tornado center, and now attached to the obstructed building, such that the tornado center is "frozen", i.e., $x_{c}(t) \equiv x_{c 0}, y_{c}(t) \equiv y_{c 0}$, while the building is viewed as "in motion" with a velocity that is equal to the original translation magnitude but opposite to the real tornado translation direction. Consequently, the interaction scenario between the tornado and the building is described as the building "virtually entering", at a translational velocity $\vec{V}_{t}=-\left(V_{t x} \vec{i}+V_{t y} \vec{j}\right)$, a purely rotational airflow $\vec{V}_{\theta}=V_{\theta}(-\vec{i} \sin \theta+\vec{j} \cos \theta)$ in which $\theta=\tan ^{-1}\left(\frac{y-y_{c 0}}{x-x_{c 0}}\right)$ becomes no longer related to the time, $t$. This new interpretation (Fig. 2.1) facilitates setting the boundary condition for the outer boundary of the tornado domain under numerical simulation, which turns out to be only the aforementioned $\vec{V}_{\theta}$ that requests no updating 
with the elapsing time. $T$, after the translation part of the tornado is associated with the building. The "translation" of the building resulting from the present RCVM can be modeled by the immersed boundary approach, which will be elucidated in Section 2.4.2. The boundary conditions afforded by this re-tailored RCVM appear both physically compatible with the flow nature and numerically much more maneuverable.

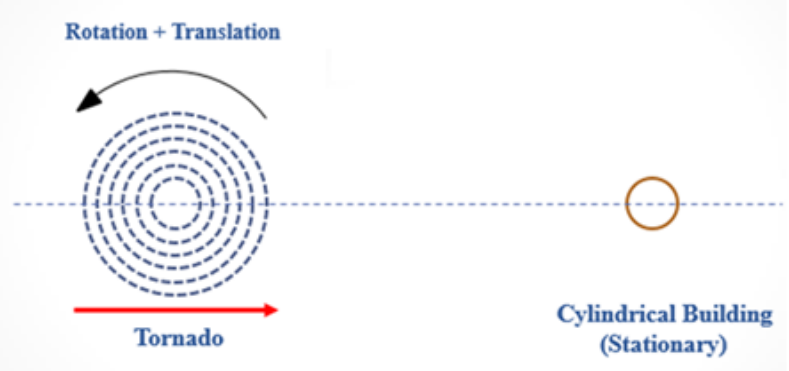

(a) Physical tornado kinematics

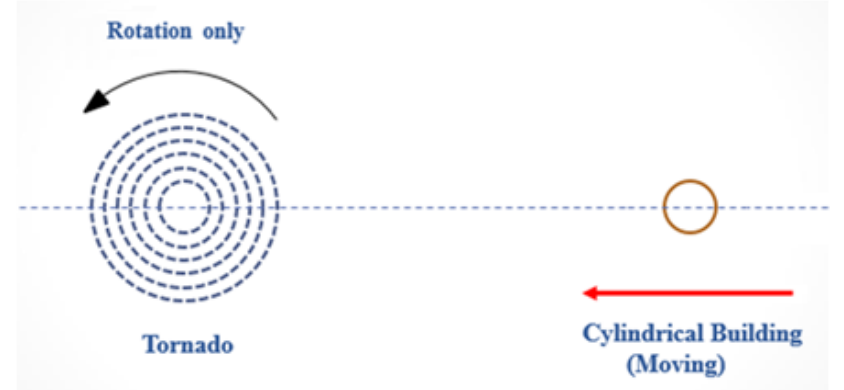

(b) Re-tailored tornado model

Fig. 2.1 Physical and re-tailored RCVM

\subsubsection{Rotation intensity in tornadic flow}

The focus of this study is placed on the investigation of the impact of intensity of the rotation 
component in a tornado-like flow, which is denoted by $\beta$ in RCVM as follows:

$$
\left(V_{\theta}\right)_{\max }=r_{c} \omega=\beta V_{t}
$$

Clearly, $\beta$ represents the ratio of the maximum tangential velociy of the rotational component, $\left(V_{\theta}\right)_{\max }$, to the translational velocity, $V_{t}$, and the larger $\beta$, the more remarkable dominancy of the rotation component in the overall tornadic flow. Detailed numerical experiments will be presented in Section 2.6, addressing the influence of rotation intensity to the aerodynamic loadings induced by a number of the tornado-like wind cases.

\subsection{Numerical method}

\subsubsection{Lattice Boltzmann model with multiple-relaxation time collision}

Unlike the traditional CFD methods, which solve the Navier-Stokes (N-S) equations numerically, lattice Boltzmann method (LBM) models the fluid consisting of fictive particles, and such particles perform consecutive streaming and collision processes based on the given discrete velocity over lattice mesh in each time step. In present study, the discrete velocities $\boldsymbol{c}_{i}$ is determined by two-dimension nine-velocity (D2Q9) model [28] (as shown in Fig. 2.2):

$$
\boldsymbol{c}_{i}= \begin{cases}(0,0) & i=0 \\ (\cos [(i-1) \pi / 2], \sin [(i-1) \pi / 2]) c, & i=1,2,3,4 \\ (\cos [(2 i-9) \pi / 4], \sin [(2 i-9) \pi / 4]) \sqrt{2} c, & i=5,6,7,8\end{cases}
$$

where $c=\delta x / \delta t . \delta x$ and $\delta t$ are lattice grid spacing and time step, respectively. The standard 
LBM takes $\delta x=\delta t=1$ (refer to Appendix for the unit conversion) with the flow domain discretized into uniform square cells. In the D2Q9 model, a particle residing at a node can travel to any one of the nearest 8 neighbor nodes at each time step along a connecting link. The particles are categorized into three types based on their speeds and moving directions. Particles of type 1 have zero speed and stay at the same node. Particles of type 2 move along the axes at a speed of $c$, whereas particles of type 3 move in the diagonal directions with a speed of $\sqrt{2} c$.

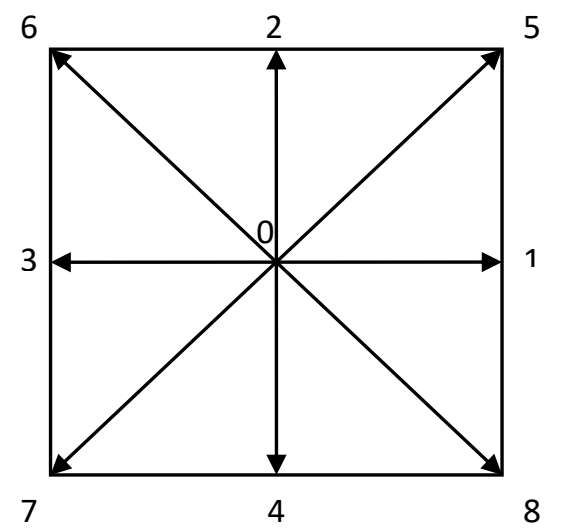

Fig. 2.2 D2Q9 model on a square lattice

The flow of a viscous incompressible fluid can be numerically simulated using the multiple-relaxation-time (MRT) LBM [39]. Originating from the lattice Boltzmann equation (LBE), the governing equation for MRT-LBM takes the following form [39]:

$$
\boldsymbol{f}\left(\boldsymbol{x}+\boldsymbol{c}_{i} \delta_{t}, t+\delta_{t}\right)-\boldsymbol{f}(\boldsymbol{x}, t)=-\mathbf{M}^{-1} \mathbf{S}\left[\boldsymbol{R}(\boldsymbol{x}, t)-\boldsymbol{R}^{e q}(\boldsymbol{x}, t)\right]
$$

where $f$ is the vector composed of distribution functions, $\mathbf{M}$ is the transformation matrix, $\mathbf{S}$ is the diagonal relaxation matrix, $\boldsymbol{R}$ is the moment vector, $\boldsymbol{R}^{e q}$ is the corresponding equilibrium 19 
moment vector. The detailed forms of these vectors and matrices are organized as follows:

$$
\begin{gathered}
\boldsymbol{f}=\left(f_{0}, f_{1}, f_{2}, f_{3}, f_{4}, f_{5}, f_{6}, f_{7}, f_{8}\right)^{T} \\
\mathbf{S}=\operatorname{diag}\left(s_{0}, s_{1}, \cdots, s_{8}\right)=\operatorname{diag}\left(0, s_{e}, s_{\varepsilon}, 0, s_{q}, 0, s_{q}, s_{v}, s_{v}\right) \\
\boldsymbol{R}=\left(\rho, e, \varepsilon, j_{x}, q_{x}, j_{y}, q_{y}, p_{x x}, p_{x y}\right)^{T} \\
\boldsymbol{R}^{e q}=\rho\left(1,-2+3 u^{2}, 1-3 u^{2}, u_{x},-u_{x}, u_{y},-u_{y}, u_{x}^{2}-u_{y}^{2}, u_{x} u_{y}\right)^{T} \\
\mathbf{M}=\left[\begin{array}{rrrrrrrrr}
1 & 1 & 1 & 1 & 1 & 1 & 1 & 1 & 1 \\
-4 & -1 & -1 & -1 & -1 & 2 & 2 & 2 & 2 \\
4 & -2 & -2 & -2 & -2 & 1 & 1 & 1 & 1 \\
0 & 1 & 0 & -1 & 0 & 1 & -1 & -1 & 1 \\
0 & -2 & 0 & 2 & 0 & 1 & -1 & -1 & 1 \\
0 & 0 & 1 & 0 & -1 & 1 & 1 & -1 & -1 \\
0 & 0 & -2 & 0 & 2 & 1 & 1 & -1 & -1 \\
0 & 1 & -1 & 1 & -1 & 0 & 0 & 0 & 0 \\
0 & 0 & 0 & 0 & 0 & 1 & -1 & 1 & -1
\end{array}\right]
\end{gathered}
$$

Note that, in the moment vector $\boldsymbol{R}, \rho$ is the density, $e$ is the energy, $\varepsilon$ is the square of the energy, $j_{x}, j_{y}$ and $q_{x}, q_{y}$ are the $x$ - and $y$-components of the momentum and the energy flux, respectively, while $p_{x x}$ and $p_{x y}$ respectively correspond to the diagonal and off-diagonal components of the viscous stress tensor [39]. In the relaxation matrix, the option of $s_{0}=s_{3}=s_{5}=0$ is permissible, and recommended for reducing the computational cost; the relaxation rates $s_{v}$ and $s_{e}$ are related to the kinematic viscosity $v$ and bulk viscosity $\zeta$ : 


$$
\begin{aligned}
& v=c_{s}^{2}\left(\frac{1}{s_{v}}-\frac{1}{2}\right) \delta_{t}, \\
& \zeta=c_{s}^{2}\left(\frac{1}{s_{e}}-\frac{1}{2}\right) \delta_{t},
\end{aligned}
$$

The relaxation rates $s_{e}, s_{q}, s_{\varepsilon}$ can be adjusted within the interval of $(0,2)$. The matrix $\mathbf{M}$ is applied to transform the distribution function $f$ onto the moment space $\boldsymbol{R}$ such that $\boldsymbol{R}=\mathbf{M} \boldsymbol{f}$ and $\boldsymbol{R}^{e q}=\mathbf{M} \boldsymbol{f}^{e q}$. The components of the corresponding equilibrium distribution function, $\boldsymbol{f}^{e q}$, are given by:

$$
f_{i}^{e q}=w_{i} \rho\left[1+\frac{\boldsymbol{c}_{i} \cdot \boldsymbol{u}}{c_{s}^{2}}+\frac{\left(\boldsymbol{c}_{i} \cdot \boldsymbol{u}\right)^{2}}{2 c_{s}^{4}}-\frac{u^{2}}{2 c_{s}^{2}}\right], i=0,1, \cdots, 8
$$

where $w_{0}=4 / 9, w_{1-4}=1 / 9, w_{5-8}=1 / 36$, and $c_{s}=c / \sqrt{3}=1 / \sqrt{3}$ representing the lattice sound speed.

The density, velocity of the fluid, and the pressure of the flow are determined as follows:

$$
\rho=\sum_{i=0}^{8} f_{i}, \quad \boldsymbol{u}=\frac{1}{\rho} \sum_{i=0}^{8} f_{i} \boldsymbol{c}_{i}, \quad p=\rho c_{s}^{2}
$$

\subsubsection{Incorporation of immersed boundary (IB) approach in LBM}

In a fluid flow problem, the interaction between the fluid and an immersed solid object can be considered as an additional forcing term, such that the computational domain becomes also inclusive of the region occupied by the solid object. This computational strategy is often referred to 
as immersed boundary (IB) method. When embedding the IB approach in LBM, the flow field $\Omega$ is represented by the Eulerian square lattices, which are the fixed Cartesian mesh points in the LBM framework; meanwhile, the boundary of the immersed object, which is denoted by $\Gamma$, is described by another set of points, $\boldsymbol{X}_{B}^{l}$, where the superscript $l$ and subscript $B$ represent the Lagrangian point and at the boundary of the immersed object, respectively. Remark that this series of points are referred to as Lagrangian points because the body itself, including its boundary, is not necessarily always at rest. An external force term reflecting the fluid-solid interaction, $f_{R S T}$, which is allocated at the Eulerian points, should be numerically determined and then seamlessly added to the LBM framework. The IB and MRT-LBM incorporation is carried out through the following procedure:

Step 1: obtain the velocity $\boldsymbol{U}_{B}^{l}$ at Lagrangian points $\boldsymbol{X}_{B}^{l}$

For measuring the closeness of an Eulerian point, $\boldsymbol{x}_{i j}=\left(x_{i}, y_{j}\right)^{t}$, and a Lagrangian point, $\boldsymbol{X}_{B}^{l}=\left(X_{B}^{l}, Y_{B}^{l}\right)^{t}$, the following continuous kernel distribution is required:

$$
D_{i j}\left(\boldsymbol{x}_{i j}-\boldsymbol{X}_{B}^{l}\right)=\delta\left(x_{i}-X_{B}^{l}\right) \delta\left(y_{j}-Y_{B}^{l}\right)
$$

with

$$
\delta(r)= \begin{cases}\frac{1}{4}\left(1+\cos \left(\frac{\pi|r|}{2}\right)\right), & |r| \leq 2 \\ 0, & |r|>2\end{cases}
$$

which is proposed by Peskin [31]. Then, based on the known velocity distribution over all Eulerian points, $\boldsymbol{u}(\boldsymbol{x}, t)$, the velocity of the fluid adhered to a Lagrangian point $\boldsymbol{X}_{B}^{l}$ can be determined by: 


$$
\boldsymbol{U}_{B}^{l}\left(\boldsymbol{X}_{B}^{l}, t\right)=\boldsymbol{u}\left(\boldsymbol{X}_{B}^{l}, t\right)=\sum_{i, j} \boldsymbol{u}(\boldsymbol{x}, t) D_{i j}\left(\boldsymbol{x}_{i j}-\boldsymbol{X}_{B}^{l}\right) \delta x \delta y
$$

with $\delta x=\delta y=1$ in the present LBM framework. Note this equation implies the satisfaction of no-slip condition at the boundary.

Step 2: Obtain the restoring force $\boldsymbol{F}_{R S T}^{l}$ at Lagrangian points $\boldsymbol{X}_{B}^{l}$

With the velocity $\boldsymbol{U}_{B}^{l}$ available at Lagrangian points, the restoring force per unit volume exerted at a Lagrangian point can be easily computed using the following feedback-forcing model [41]:

$$
\boldsymbol{F}_{R S T}^{l}=\alpha_{1} \int_{0}^{t}\left(\boldsymbol{U}_{B}^{l}-\boldsymbol{U}_{I M S}\right) d t+\alpha_{2}\left(\boldsymbol{U}_{B}^{l}-\boldsymbol{U}_{I M S}\right)
$$

In Eq. (2.19), $\alpha_{1}$ and $\alpha_{2}$ are two negative free constants with dimensions of $M L^{-3} T^{-2}$ and $M L^{-3} T^{-1}$, respectively. According to [41], $\alpha_{1}$ and $\alpha_{2}$ are stable for moderate values within the interval $[-100,-1]$, and neither is sensitive to its exact value. $\boldsymbol{U}_{I M S}$ in Eq. (2.19) stands for the moving velocity of the immersed body itself. In the case of an immobile immersed body, $\boldsymbol{X}_{B}^{l}=\boldsymbol{X}_{B}^{l}(s)$, in which $s$ denoting the Lagrangian parametric coordinate, thus, $\boldsymbol{U}_{I M S} \equiv \mathbf{0}$; if the immersed body moves in the fluid, then,

$$
\boldsymbol{X}_{B}^{l}=\boldsymbol{X}_{B}^{l}(s, t)
$$

and

$$
\boldsymbol{U}_{I M S}(s, t)=\frac{\partial \boldsymbol{X}(s, t)}{\partial t}
$$


which can be numerically determined using:

$$
\boldsymbol{U}_{I M S}(s, t)=\frac{\boldsymbol{X}(s, t)-\boldsymbol{X}(s, t-\delta t)}{\delta t}
$$

Step 3: Obtain the restoring force $\boldsymbol{f}_{R S T}$ at Eulerian points $\boldsymbol{x}_{i j}$

The restoring force has to be expressed in the Eulerian framework, i.e., at the Cartesian grid points, so that this additional force can be seamlessly incorporated in MRT-LBM. Compared to the transformation described in Eq. (2.18), now the transformation from $\boldsymbol{F}_{R S T}^{l}\left(\boldsymbol{X}_{B}^{l}, t\right)$ to $\boldsymbol{f}_{R S T}\left(\boldsymbol{x}_{i j}, t\right)$ reversely unfolds as follows:

$$
\boldsymbol{f}_{R S T}\left(\boldsymbol{x}_{i j}, t\right)=\sum_{l} \boldsymbol{F}_{R S T}^{l}\left(\boldsymbol{X}_{B}^{l}, t\right) D_{i j}\left(\boldsymbol{x}_{i j}-\boldsymbol{X}_{B}^{l}\right) \Delta s_{l}
$$

where $\Delta s_{l}$ is the arc length of a boundary element.

Step 4: Embed the restoring force $f_{R S T}$ in LBM

In order to invoke $f_{R S T}$ in the LBM framework, the following transformation is employed:

$$
F_{i}=\left(1-\frac{1}{2 \tau}\right) w_{i}\left(\frac{\boldsymbol{c}_{i}-\boldsymbol{u}}{c_{s}^{2}}+\frac{\boldsymbol{c}_{i} \cdot \boldsymbol{u}}{c_{s}^{4}} \boldsymbol{c}_{i}\right) \cdot \boldsymbol{f}_{R S T}
$$

with $w_{i}$ representing the weighting coefficients in the equilibrium distribution function as aforementioned for Eq. (2.14), such that the additional forcing term, $F_{i}$, can be included into the right hand side, leading to the following revised LBE [40]:

$$
f_{i}\left(\boldsymbol{x}+\boldsymbol{c}_{i} \delta t, t+\delta t\right)-f_{i}(\boldsymbol{x}, t)=-\frac{1}{\tau}\left(f_{i}(\boldsymbol{x}, t)-f_{i}^{e q}(\boldsymbol{x}, t)\right)+F_{i} \delta t
$$


Remark that, accordingly, the velocity in the LBM framework should also take $\boldsymbol{f}_{R S T}$ into account:

$$
\rho \boldsymbol{u}=\sum_{i=0}^{8} f_{i} \boldsymbol{c}_{i}+\frac{1}{2} \boldsymbol{f}_{R S T} \delta t
$$

\subsubsection{Smagorinsky subgrid-scale (SGS) model}

In order to enhance the numerical stability at elevated Reynolds number in present study, LES on the basis of Smagorinsky subgrid-scale (SGS) model [22] is embedded in the MRT-IB-LBM framework. The spirit of large-eddy-based LBM is to split the total viscosity $v$ into two parts: the molecular viscosity $v_{0}$ and the eddy viscosity $v_{t}$. Analogously, the total relaxation time is defined as:

$$
\tau=\tau_{0}+\tau_{t}=\tau_{0}+\frac{v_{t}}{c_{s}^{2} \delta_{t}}
$$

The molecular viscosity can be obtained via the definition of $\mathrm{Re}$, and eddy viscosity $v_{t}$ is defined as:

$$
v_{t}=\left(C \Delta_{L}\right)^{2} \sqrt{2 \vec{S}_{i j} \vec{S}_{i j}}
$$

where the adjustable constant $C$ is called the Smagorinsky constant and is fixed as 0.1 in present study. $\Delta_{L}$ is the filter width. $\vec{S}_{i j}$ represents the strain rate tensor, which can be expressed as:

$$
\vec{S}_{i j}=\frac{1}{2}\left(\frac{\partial \vec{u}_{i}}{\partial x_{j}}+\frac{\partial \vec{u}_{j}}{\partial x_{i}}\right)
$$


Then, the strain rate tensor is evaluated from non-equilibrium properties of the filtered particle distribution function, which can be computed as:

$$
\vec{S}_{i j}=-\frac{3}{2 \rho_{0} \tau} \vec{Q}_{i j}
$$

where the momentum flux tensor $\vec{Q}_{i j}=\sum_{\alpha} e_{\alpha i} e_{\alpha j}\left[f_{\alpha}-f_{\alpha}^{e q}\right]$.

Mathematically combining Eqs. (2.27), (2.28) and (2.30), the total relaxation time $\tau$ is obtained as:

$$
\tau=\frac{1}{2}\left(\tau_{0}+\sqrt{\tau_{0}^{2}+\frac{2}{\rho \delta t c_{s}^{4}}\left(C \Delta_{L}\right)^{2}|Q|}\right)
$$

where $|Q|=\sqrt{2 \vec{Q}_{i j} \vec{Q}_{i j}}$.

\subsection{Validation}

As aforementioned, very little literature on numerical simulation of tornado-induced wind load on buildings can be found; in particular, those using RCVM as model basis are even fewer. Although the RCVM-based model developed in this study features co-existence of rotational and translational components, for the purpose of making a feasible and yet indicative comparison, a practical option is to switch one component on while turning off the other component, such that the rotational and translational flow results from the present IB-LBM approach can be at least separately compared against a variety of available relevant results that can be easily found in [43]-[45].

The employed IB-LBM framework has been successfully validated as an approach owned 
second-order accuracy in [49], furthermore, grid independent study was conducted in [49] as well, which indicated that the results were grid-independent when grid resolution reaches the level of 1/40. All IB-LBM simulations performed in this study use a uniform grid based on Cartesian coordinates, and the corresponding time step size is 1 . The nominal fluid density is set at $\rho=1.0$, the translation velocity, $V_{t}$, and the cylinder diameter, $D$, are taken as the characteristic velocity and length when defining Reynolds number $(\mathrm{Re})$ as $\operatorname{Re}=\frac{V_{t} D}{v}$. In order to investigate flows with elevated Reynolds number, the Smagorinsky sub-grid stress (SGS) model [22] is incorporated in the present framework.

\subsubsection{Rotational Couette flow}

The first comparison subject is a purely rotational flow, which is also referred to as rotational Couette flow. The flow domain is in a "ring" shape, which is "sandwiched" by an inner cylinder and an outer cylindrical wall that are coaxial and rotating at two different speeds. An analytical solution of the laminar circular Couette flow is expressed as [42]:

$$
V_{\theta}(r)=C_{1} r+\frac{C_{2}}{r}
$$

with factors

$$
C_{1}=\frac{\Omega_{2} R_{2}^{2}-\Omega_{1} R_{1}^{2}}{R_{2}^{2}-R_{1}^{2}}, C_{2}=\frac{\left(\Omega_{1}-\Omega_{2}\right) R_{1}^{2} R_{2}^{2}}{R_{2}^{2}-R_{1}^{2}}
$$

The commercial ANSYS FLUENT software package switched to the option of the 27 
Navier-Stokes flow model and the present IB-LBM code are both employed, and the two sets of rotational flow simulation results will be compared to the analytical solution for validation purpose. For both simulations, a stationary cylinder of one-unit diameter is placed at the center of the 2D computational domain, with no-slip boundary condition strictly satisfied on the surface of the cylinder. Based on RCVM, set $\omega=1.5 \mathrm{rad} / \mathrm{s}, r_{c}=3$ units, where the peak tangent velocity, $\left(V_{\theta}\right)_{\max }$, physically arises. Accordingly, by substituting $R_{1}=0.5$ unit, $R_{2}=r_{c}=3$ units, $\Omega_{1}=0$, and $\Omega_{2}=\omega=1.5 \mathrm{rad} / \mathrm{s}$ into Eqs. (2.32) and (2.33), the analytical solution of this flow within the "ring" area becomes:

$$
V_{\theta}(r)=\frac{\Omega_{2} R_{2}^{2}}{R_{2}^{2}-R_{1}^{2}} \cdot \frac{r^{2}-R_{1}^{2}}{r}=\frac{27}{70} \cdot \frac{4 r^{2}-1}{r}
$$

In the numerical simulations, the imposition of $\left(V_{\theta}\right)_{\max }=r_{c} \omega$, which is according to RCVM, is constantly maintained at the circular line with $r=r_{c}$ when using FLUENT; in the present IB-LBM run, $\left(V_{\theta}\right)_{\max }$ needs to be imposed at that circular line with $r=r_{c}$ only at the beginning of computation. In order to ensure the accordance with RCVM at the outer boundary of the rectangular computational domain, the velocity boundary condition in this test is based on:

$$
V_{\theta}=\frac{\omega r_{c}^{2}}{r}=\frac{27}{2} \cdot \frac{1}{\sqrt{x^{2}+y^{2}}}
$$

which is the local tangential velocity component with $\omega=1.5 \mathrm{rad} / \mathrm{s}$ and $r_{c}=3$ units taken into consideration, while nil local radial velocity component still needs to be retained at the outer 
boundary. Hence, the rectangular flow domain in this case can be divided into two zones: of key interest is the velocity solution within the "ring" area, which is the core zone and features the laminar circular Couette flow; the off-core zone, which is the outside of the "ring" area, is governed by the boundary condition of RCVM type and no longer within the circular Couette flow context.

The velocity magnitude contours from the two simulations are shown in Fig. 2.3, confirming the purely tangential flow nature of the rotational Couette flow. Detailed tangent velocity profiles along a radial line obtained by the two simulations are both depicted in Fig. 2.4 and compared against the available analytical solution [42]. The first range with increasing tangent velocity contains slight difference between the three solutions, the IB-LBM solution looks overall closer to the analytic solution than the N-S solution resulting from FLUENT. No visible difference between the three solutions can be detected in the second range with decreasing tangent velocity, indicating the RCVM analytic method, the present IB-LBM approach, and the Navier-Stokes flow model can converge in this off-core flow zone when the latter two employ RCVM for boundary condition use.

Remark also that neither Eq. (2.34) nor (2.35) shows the tangent velocity component has an explicit connection to the viscosity of the particular rotating fluid and, in turn, the Reynolds number (Re) of the flow. Misleadingly, this might have suggested one to think that, in a tornadic wind, the flow characteristics depends mainly on its translational flow part, $V_{t}$. At present, the tornado researchers have conventionally taken the incoming translational velocity component as characteristic velocity in determining the Reynolds number of a tornadic wind. However, both Eq. 
(2.34) and (2.35) indicate that, from an analytical point of view, the angular velocity in the core zone of a tornado, $\Omega_{2}$ in the Circular Couette flow formula, i.e., $\omega$ in RCVM, plays a crucial role in the magnitude of local tangent velocity component, $V_{\theta}$, and in turn, the overall flow field, given that $\vec{V}=\vec{V}_{t}+\vec{V}_{\theta}$ holds in the entire tornadic wind field as indicated in Eq. (2.2). In fact, this angular velocity reflects the rotation intensity in a tornadic wind, $\beta$, as demonstrated in Eq. (2.4). In Section 2.6, the impact of this rotation intensity will be investigated in detail.
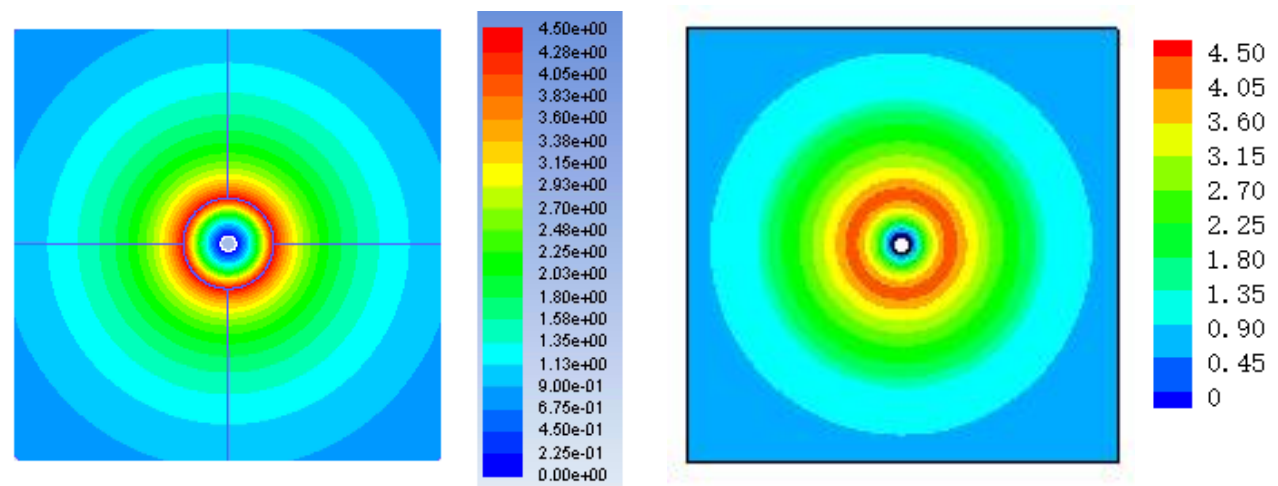

Fig. 2.3 Velocity magnitude contours (left: using ANSYS FLUENT; right: using LBM)

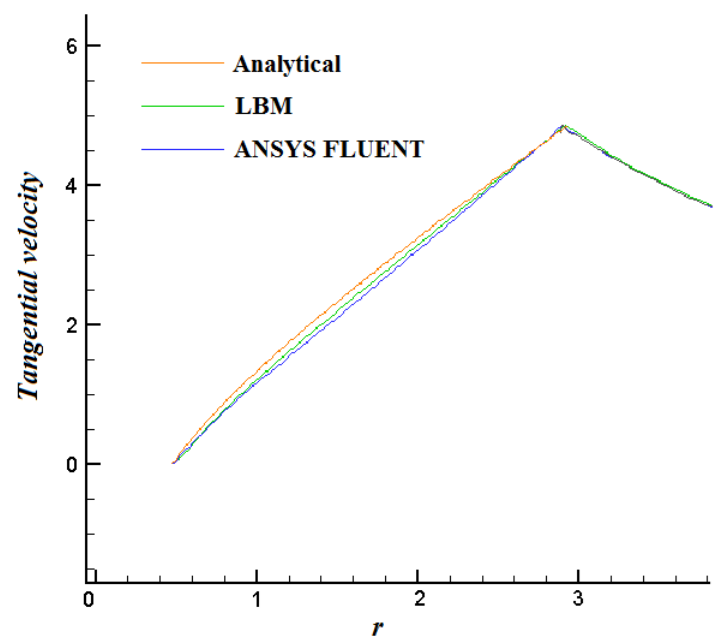

Fig. 2.4 Tangent velocity profile comparison [42] 


\subsubsection{Translational flow at elevated Reynolds number}

Now, a series of purely translational flows over a stationary cylinder at Re from 1000 to 3900 are examined here to validate the applicability of the present IB-LBM code to simulation of flows at relatively high Reynolds numbers. The computational domain is $[-40,40] \times[-12.5,12.5]$ rectangular domain. The cylinder is fixed at $(0,0)$, and mesh resolution is $1 / 50$. This sort of tests has been extensively studied using other numerical methods, and some existing results reported elsewhere will serve here as references for comparison use.

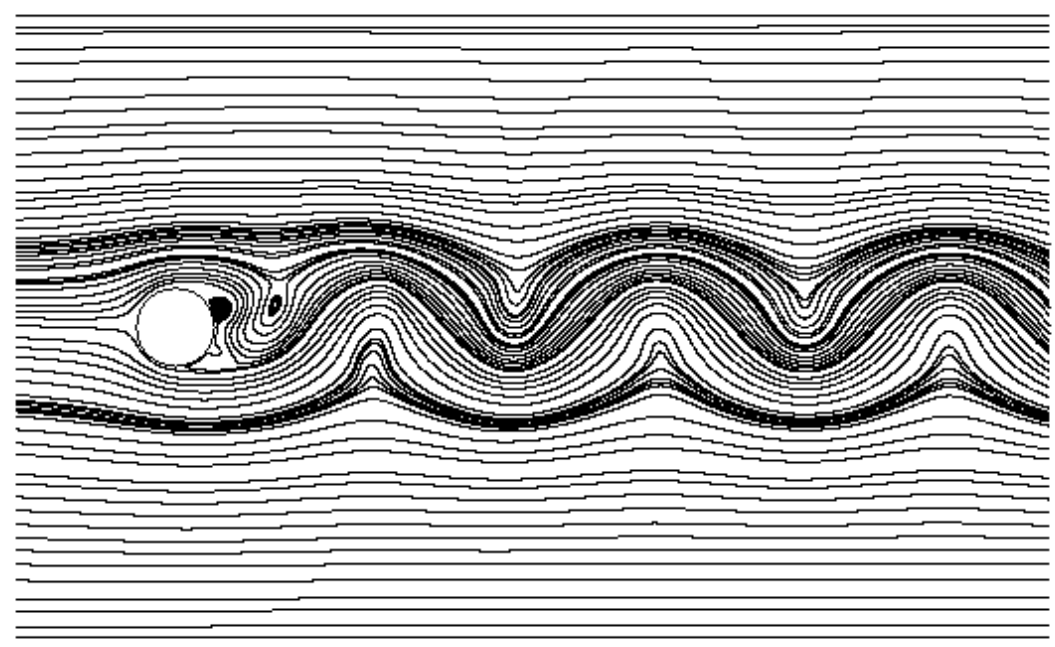

Fig. 2.5 Streamlines around cylinder at $R e=1000$ 


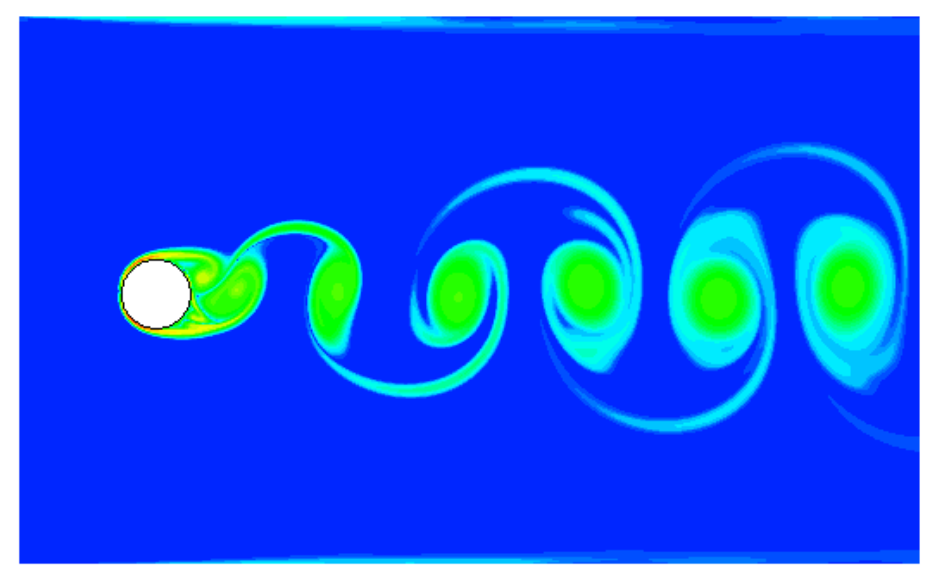

Fig. 2.6 Vorticity around cylinder at $R e=1000$

Fig. 2.5 and Fig. 2.6 illustrate the instantaneous streamlines and vorticity contours at $\mathrm{Re}=1000$ in the vicinity of a cylinder, respectively, and the Karman vortex street is seen in both figures. In particular, Fig. 2.6 clearly reveals that the vortex is shedding at a constant frequency. Here, the Strouhal number is employed to obtain the dimensionless frequency when the vortices are shed from the body:

$$
S t=\frac{f_{q} D}{V_{t}}
$$

with $f_{q}$ representing the vortex shedding frequency. This frequency can be acquired by examining the time evolution of, for instance, the drag and lift coefficients. The drag coefficient is defined as:

$$
C_{d}=\frac{F_{D}}{0.5 \rho V_{t}^{2} D}
$$

where $F_{D}$ is the drag force, and can be calculated [31] by: 


$$
F_{D}=-\int_{\Omega} f_{x} d v
$$

where $f_{x}$ stands for the $x$-component of force density $\boldsymbol{F}_{R S T}$ calculated in Eq. (2.19) at the boundary point. Similarly, the lift coefficient can be defined as:

$$
C_{l}=\frac{F_{L}}{0.5 \rho V_{t}^{2} D}
$$

where $F_{L}$ is the lift force obtained by [31]:

$$
F_{L}=-\int_{\Omega} f_{y} d v
$$

in which $f_{y}$ represents the $y$-component of the force density $\boldsymbol{F}_{R S T}$.

The drag and lift coefficients demonstrated in Fig. 2.7 looks both periodically oscillating, which essentially respond to the periodic shedding of vortices from the cylinder. A detailed comparison of the present drag coefficient and Strouhal number against other available data, as shown in Table 2.1, shows the results obtained from this study are within the range of values reported in other references. Moreover, as demonstrated in Fig. 2.8, using the present LBM based approach, the drag coefficient ranging from $\mathrm{Re}=1000$ to 3900 increases monotonically and, again, agrees pretty well with the data resulting from other methods. This indicates that present IB-LBM framework with the aid of LES can achieve reliable simulation results at elevated Reynolds numbers. On the other hand, this series of preliminary tests reveal that the mean drag coefficient goes up monotonically when the Reynolds number rises in this testing range while the flow is purely translational, i.e., the rotation 
intensity is nil $(\beta=0)$.

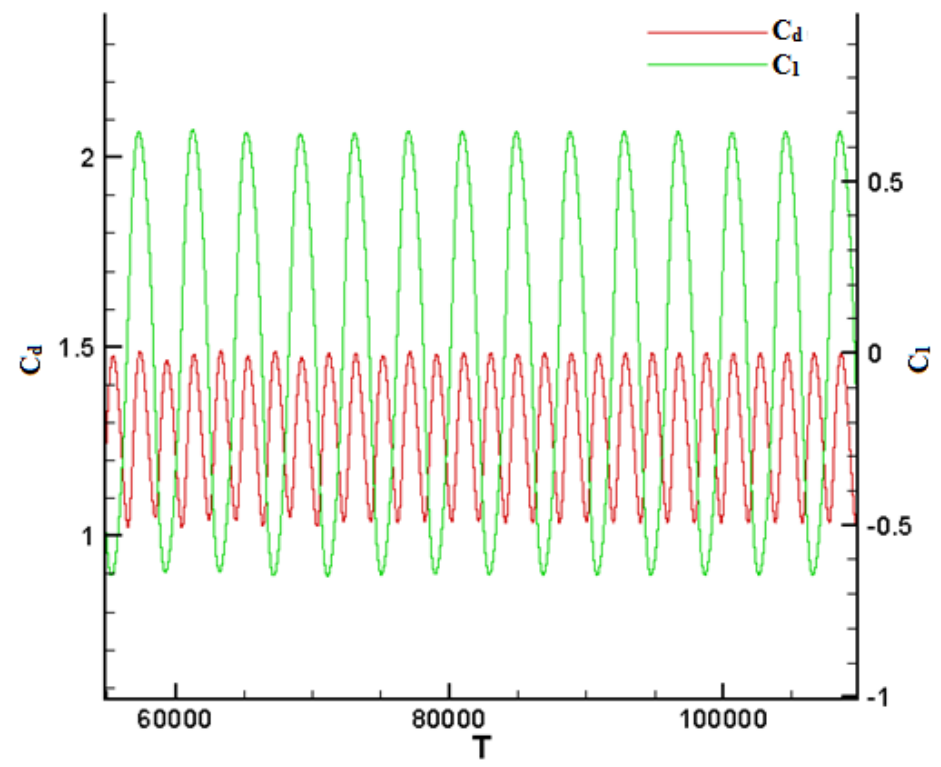

Fig. 2.7 Evolution of drag and lift coefficients at $R e=1000$

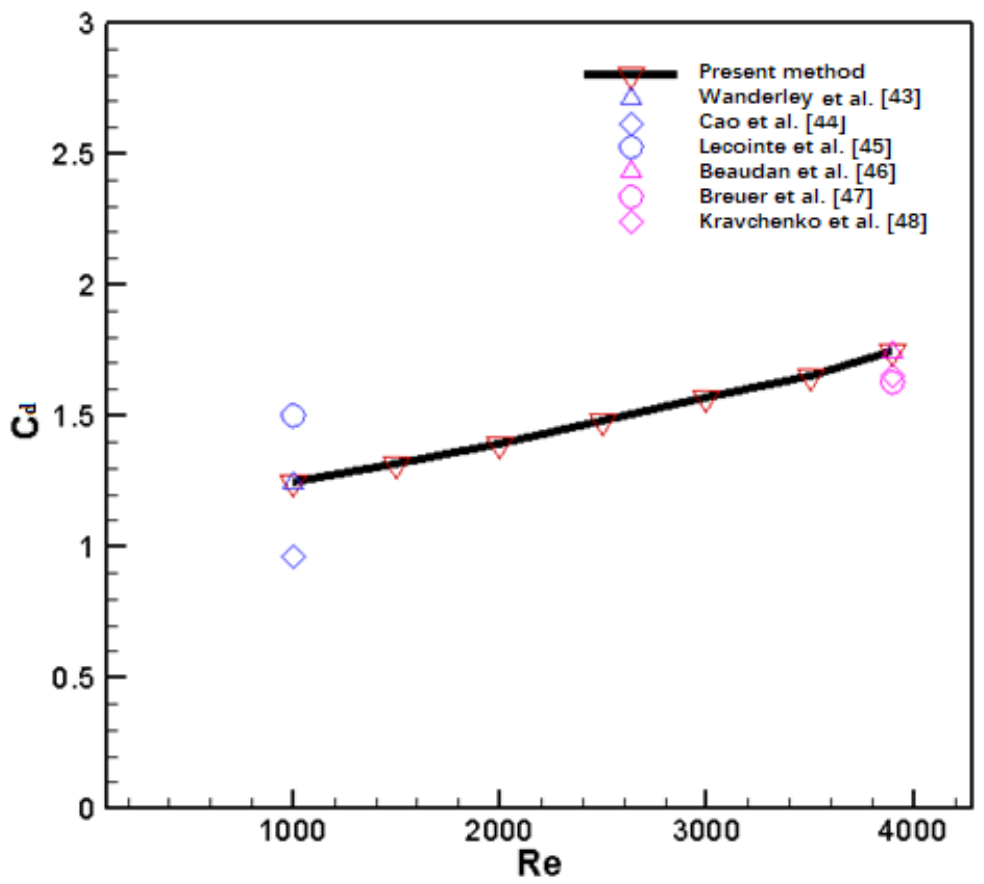

Fig. 2.8 Evolution of mean drag coefficient and comparison with references 
Table 2.1 Comparisons of mean drag coefficient and Strouhal number at $R e=1000$

\begin{tabular}{lcc}
\hline References & Drag coefficient & Strouhal number \\
\hline Present method & 1.25 & 0.238 \\
COMSOL Multiphysics & 1.20 & 0.225 \\
& 0.96 & 0.193 \\
Wanderley et al. [43] & 1.24 & 0.206 \\
Cao et al. [44] & 1.50 & 0.24 \\
\hline
\end{tabular}

\subsection{Numerical experiments}

Following the validation of the present IB-LBM framework for simulation of purely rotational and translational flows in Section 2.5, this approach is now employed to investigate the impact of the rotation intensity in a tornado-like airflow over a construction. As described in Section 2.3.2, the scenario of a tornado passing over a building is interpreted as a superposition of a "locked" rotational airflow and a "virtual translation" of the building, which is re-tailored from the original RCVM in order to render the outer boundary condition time-independent.

In all subsequent simulations, assign $D=20 \mathrm{~m}$ and $V_{t}=20 \mathrm{~m} / \mathrm{sec}$ (towards the positive $x$-direction) for the tornado-construction interaction scenario under numerical investigation. If taking 
$D$ as 1 unit of length (50 lattices with 1/50 mesh resolution), then the computational domain occupies a $[-40,40] \times[-12.5,12.5]$ rectangular domain. Using the re-tailored RCVM, the center of the rotational flow component is fixed at $(0,0)$, and the initial position of the building center is located at $(35,0)$ with a translational speed at $V_{t}=0.034$ (refer to Appendix for the unit conversion) towards the negative $x$-direction, which corresponds to a physical scenario in which the tornado is both translating in the positive $x$-direction and rotating while the building keeps locked and initially is 35 units away from the center of the incoming tornado.

Three characteristic coefficients, $C_{x}, C_{y}$, and $C_{m}$, which reflect the aerodynamic force components in the $\mathrm{x}$ - and $\mathrm{y}$-directions, $F_{x}, F_{\mathrm{y}}$, and the resulting moment, $M$, respectively, are defined as:

$$
\begin{gathered}
C_{x}=\frac{F_{x}}{0.5 \rho V_{t}^{2} D} \\
C_{y}=\frac{F_{y}}{0.5 \rho V_{t}^{2} D} \\
C_{m}=\frac{M}{0.5 \rho V_{t}^{2}\left(\pi D^{2} / 4\right)}
\end{gathered}
$$

and will be used for examining the wind loading on the cylindrical building with diameter $D$. Note that force coefficients $C_{x}, C_{y}$ are the same as the drag and lift coefficients defined in Eq. (2.37) and Eq. (2.39), respectively. To analyze the flow characteristics in a more straightforward nomenclature, 
$C_{x}, \mathrm{C}_{\mathrm{y}}$ will be used in the following tornado interpretation. The moment $M$ is defined as $\int_{\Omega}\left(\mathbf{r} \times \boldsymbol{F}_{R S T}\right) d v$

\subsubsection{An example}

Before focusing on the effects of the rotation intensity in a tornadic wind scenario, a case at $R e$ $=1000, r_{c}=3$ units, and with rotation intensity specified at $\beta=0.5$, i.e., $\left(V_{\theta}\right)_{\max }=r_{c} \omega=0.5 V_{t}$, according to Eq. (2.4), is employed as an example to illustrate the key aspects of numerical investigation. For the sake of description convenience, three representative time points during the tornado-building interaction are chosen for detailed observation, namely, (1) the tornado core zone reaches the building, i.e., $x_{\text {building_center }}-x_{\text {tornado_center }}=r_{c}$, which is referred to as "core-in" time; (2) the tornado and building centers are coinciding, i.e., $x_{\text {building_center }}=x_{\text {tornado_center }}$, which is referred to as "coinciding-center" time; and (3) the tornado core zone leaves the building, i.e., $x_{\text {tornado_center }}-x_{\text {building_center }}=r_{c}$, which is referred to as "core-out" time. Remark that the names of these three time points do not suggest the onset of physical phenomena in their exact sense since the interaction between the tornadic wind and building physically takes place when $x_{\text {building_center }}-x_{\text {tornado_center }}>r_{c}$, and rotation and translation are concurrent all the way in a tornadic wind event. Between the "core-in" and "core-out" time points is the primary stage of the tornado-building interaction, whereas before and after the primary stage are respectively referred to as pre-interaction and post-interaction stages. Fig. 2.9- Fig. 2.11 depicted the streamline pattern and 
velocity magnitude at the three representative time points, respectively.

As demonstrated in Fig. 2.9, until the core-in time point, the rotational flow pattern appears overall retained except in the vicinity of the building. This indicates that, in the pre-interaction stage, the tornado-building interaction has not been significant yet, and the affected zone remains very restricted, mainly behind the cylinder where an eddy arises, demonstrating the dominance of the translational flow component towards the obstacle. During the primary interaction stage, Fig. 2.10 shows the tornado core area is strongly disturbed since a few eddies take place in the core zone while beyond the core zone the streamlines overall remain encircling the building. In Fig. 2.11, the severely disturbed flow featuring co-occurrence of a number of eddies is found in the wake region.

By comparing velocity magnitude in Fig. 2.9- Fig. 2.11, the highest flow rate is found right in front of the cylinder in the pre-interaction stage; then, in the primary and post-interaction stages, the zone of highest flow rate is located in the wake region, and this fastest flow zone goes more distant from the building when time further elapses. Also, Fig. 2.11 reveal that vortices are shedding in the wake area, suggesting the re-occurance of the dominant translational flow component upon recalling the renowned Karman vortex street phenomenon that arises in the case of a cylinder facing a purely translational incoming flow with an elevated Reynolds number. On the other hand, all these six figures exhibit a slight upward trend for the wake region, which is attributed to the counterclockwise rotational flow component in this tornadic wind. All observed characteristics echo the translation and rotation superposition nature of this flow. 

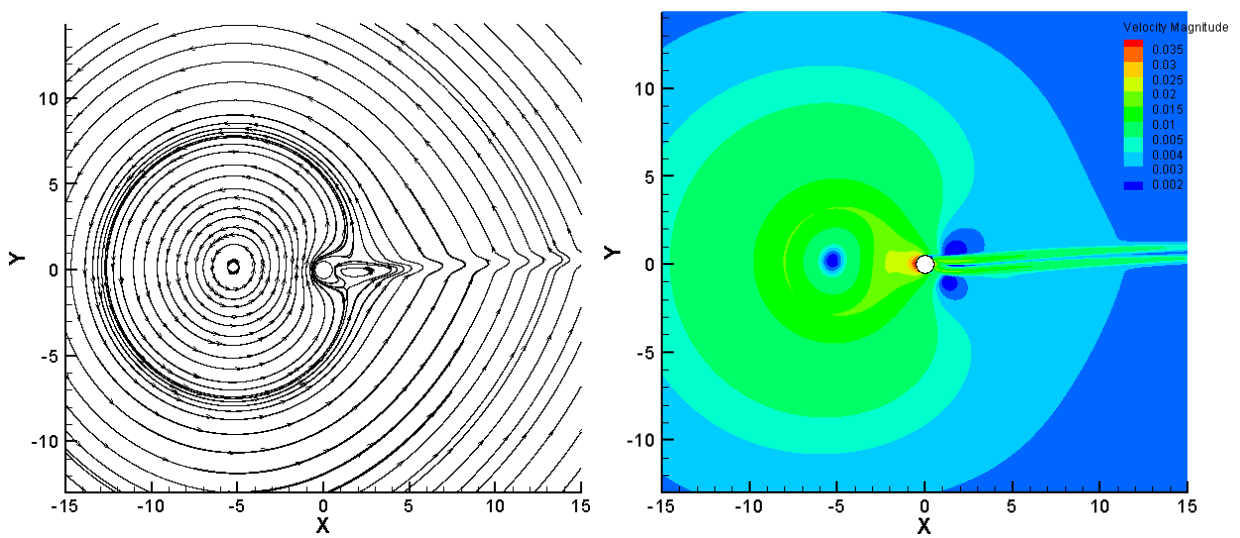

Fig. 2.9 Streamlines and velocity magnitude at "core-in" time
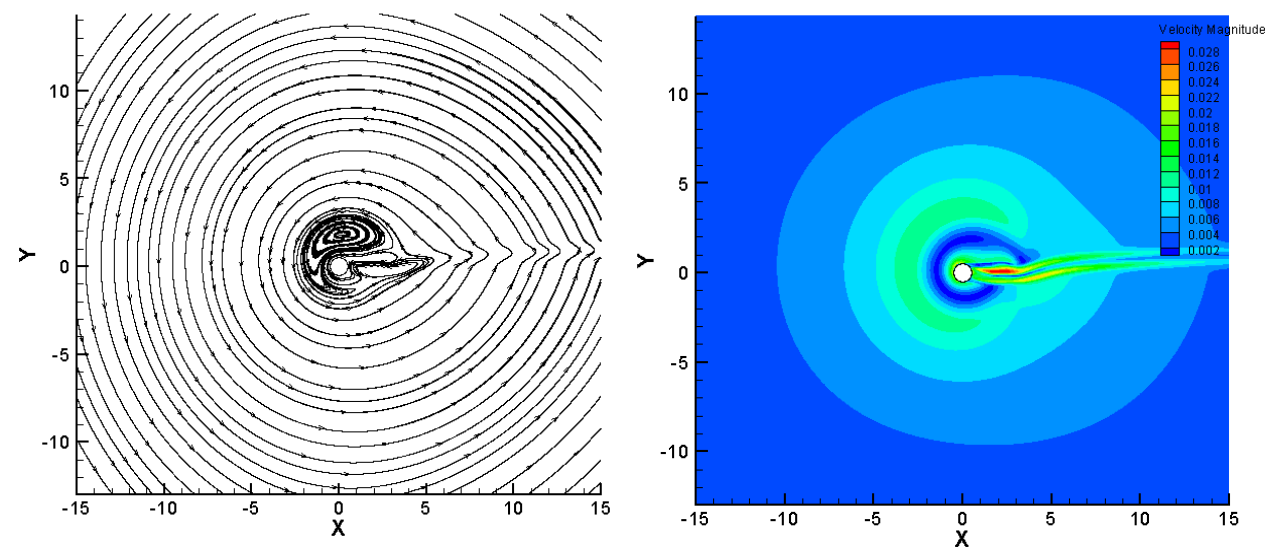

Fig. 2.10 Streamlines and velocity magnitude at "coinciding-center" time
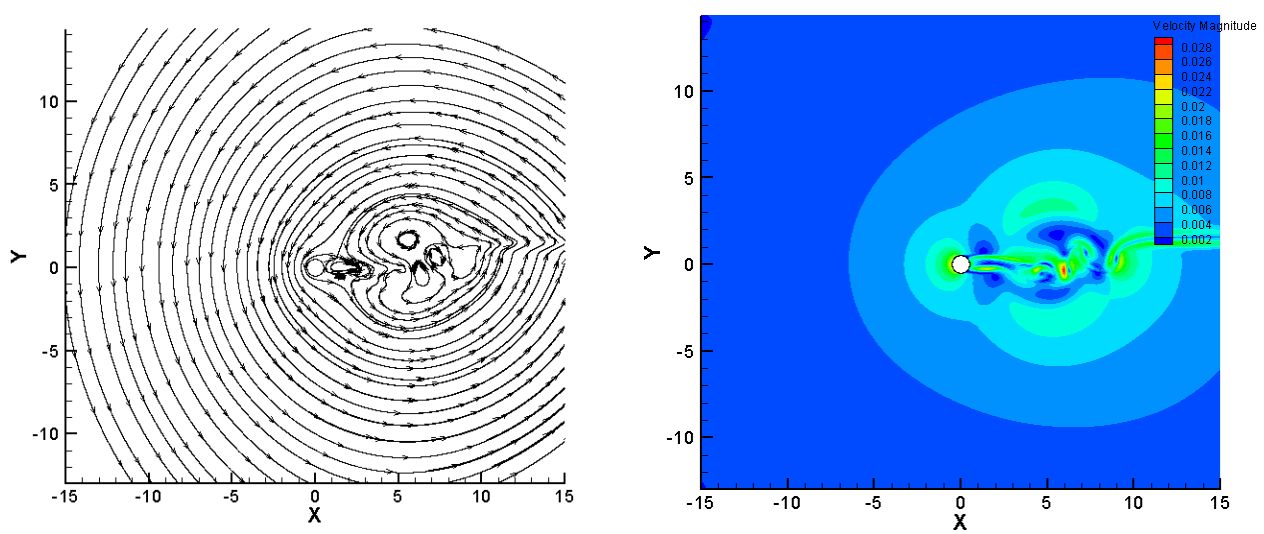

Fig. 2.11 Streamlines and velocity magnitude at "core-out" time

Besides the qualitative analysis based on the streamline and velocity magnitude contours, 
quantitative analysis through evolution of $C_{x}, C_{y}$, and $C_{m}$, as defined in Eqns. (2.41) - (2.43), can be also made. Next, a series of tornadic flow simulations aimed at investigation of the effect of the rotation intensity will be conducted, and detailed analysis of the coming new cases will be demonstrated with the aid of the three aerodynamic coefficients.

\subsubsection{Tornado-like wind induced loads on a cylinder}

After interpreting the overall flow features through streamlines and velocity magnitude plottings physically, more details about how rotation intensity can affect the aerodynamic coefficients will be examined and discussed as follows.

Unlike in Section 2.5.2 where the cases examined the purely translational flows (by locking the rotation intensity at $\beta=0$ ) around a single cylinder with Reynolds number ranging from 1000 to 3900 (through adjusting the fluid viscosity while fixing the characteristic velocity $V_{t}$ ), the tornadic wind composed of both translational and rotational flow components is the examination subject, and the investigation focus is now placed on the effect of rotation intensity. Recall that Section 2.5.2 demonstrated that in the Re range of 1000 to 3900 , the "drag" coefficient, $C_{d}$, monotonically increases when Re goes up. In this section, the three aerodynamic coefficients, $C_{x}, C_{y}$, and $C_{m}$, are

all examined for different rotation intensities, $\beta=0.5,1$, and 2 ; for each rotation intensity test series, Reynolds number starts from 1000 and then continuously increases, aiming to examine whether the wind loadings monotonically increase when $R e$ rises. 
2.6.2.1 Test series 1: $r_{c} \omega=0.5 V_{t} \quad(\beta=0.5)$

In this test series, $\beta=0.5$ indicates the maximum local tangent velocity component due to the rotation component is only half of the incoming translational velocity component, hence, this flow is overall mainly dominated by the translational part. Fig. 2.12 - Fig. 2.14 respectively group the evolutions of $C_{x}, C_{y}$, and $C_{m}$ over the Re testing range from 1000 to 3900 . In these three figures, the $x$-coordinate $(\mathrm{X})$ denotes the relative distance between tornado center and the cylinder, and thus position $x=0$ on the horizontal axis represents the time instant at which the centers of the tornado and cylinder are nominally coinciding if solely taking into account the translation of the tornado center; in the intervals $x<0$ and $x>0$, the tornado is approaching and leaving the cylinder, respectively. An overall observation of the three figures may find out two major turning points on the horizontal axis approximately located at $x=-3$ ("core-in" time) and $x=3$ ("core-out" time).

In the pre-interaction stage $(x<-3), C_{x}$ and $C_{m}$ decrease while $C_{y}$ increases, indicating the cylinder is pulled towards the tornado center in the $x$-direction, uplifting in the $y$-direction, and twisted in the clockwise direction. Recall that, in this case, $\beta=0.5, V_{t}=0.034$ unit/sec, and $r_{c}=3$ units. In Fig. 2.12 - Fig. 2.14, when $x<-3$, the distance between the tornado center and the cylinder center, $r$, is greater than $r_{c}$. According to RCVM as expressed in Eq. (2.1), $V_{\theta}=r_{c}^{2} \omega / r=\beta V_{t} \frac{r_{c}}{r}$ when $r>r_{c}$. Thus, the local upward tangent velocity component (due to counterclockwise rotation in the tornadic wind) for the left half of the cylinder is larger than that for 
the right half, leading to a larger uplifting force exerted in the left half of the cylinder than that for the right half. This yields the overall clockwise twisting effect for this pre-interaction stage. Also, a distinct point is approximately located at $x=-10$, as the overall variation slopes for the intervals $x<$ -10 and $-10<x<-3$ are significantly different for each of Fig. $2.12-$ Fig. 2.14, the former being smaller while the latter being larger. The steep change of the three coefficients within $-10<x<-3$ can be interpreted again by RCVM, as the aerodynamic forces and moment are related to $V_{\theta}$ while, in $V_{\theta}=\beta V_{t} \frac{r_{c}}{r}, \beta, V_{t}$, and $r_{c}$ are all constants, the slope of $V_{\theta}$ is proportional with $\frac{1}{r^{2}}$, which becomes more considerable with decreasing $r$ while diminishing once $r>10$.

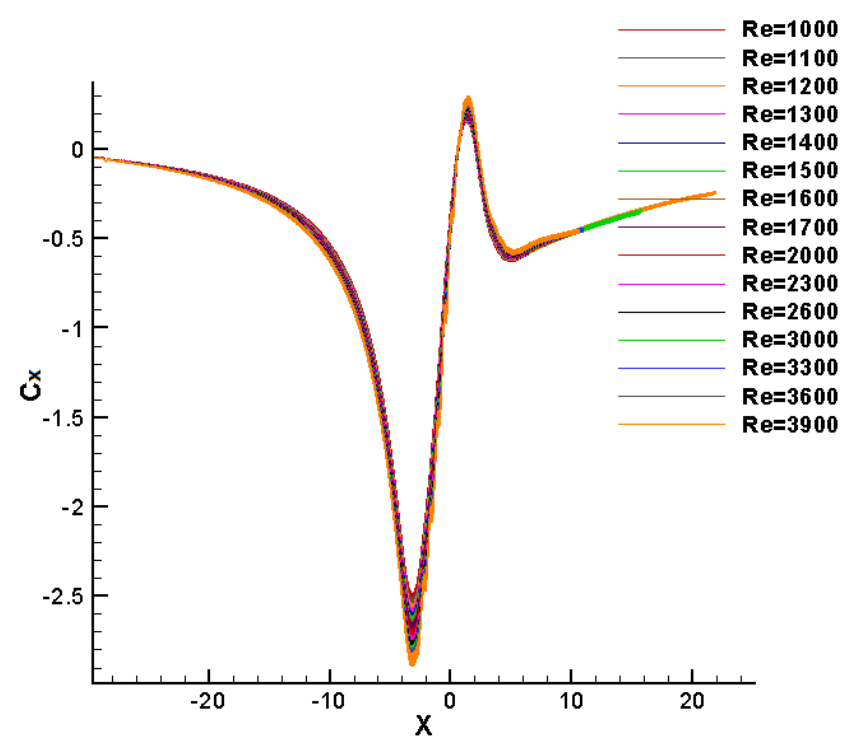

Fig. 2.12 $C_{x}$ evolutions for different Reynolds numbers at $\beta=0.5$ 


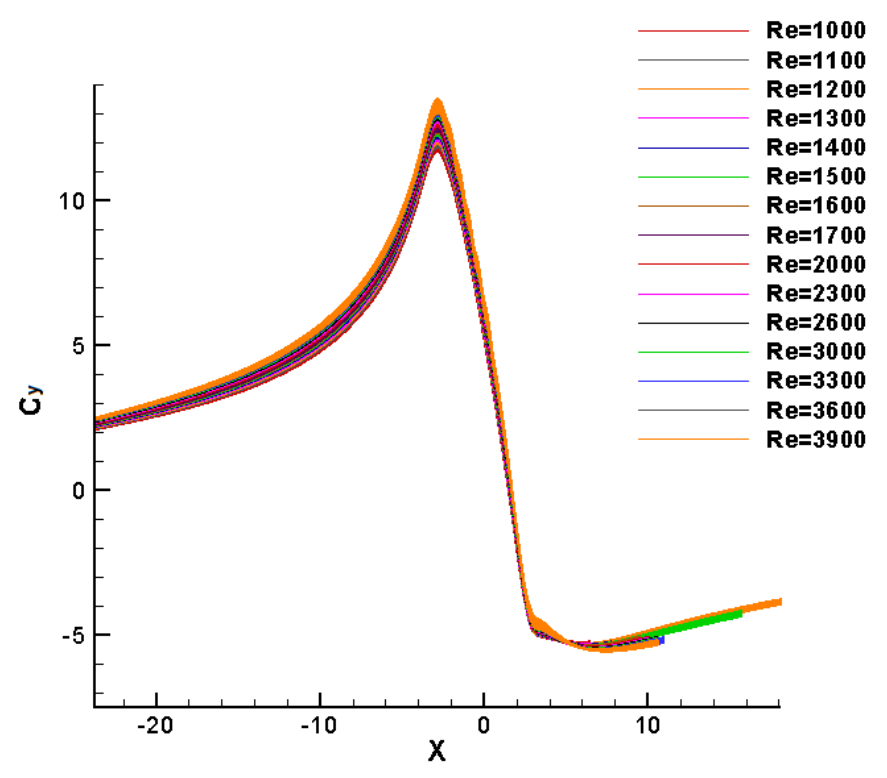

Fig. 2.13 $C_{y}$ evolutions for different Reynolds numbers at $\beta=0.5$

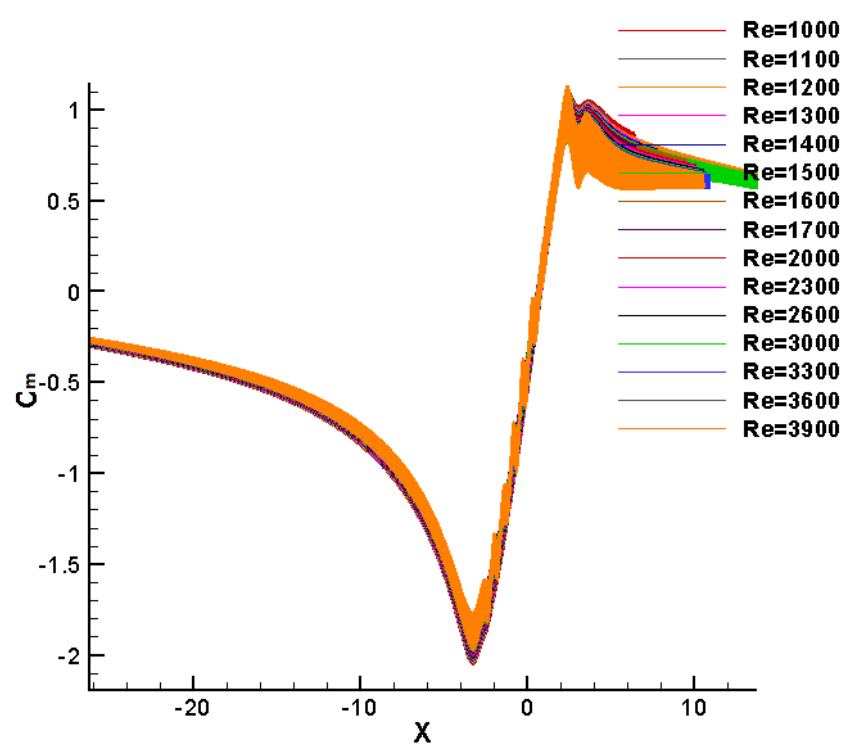

Fig. 2.14 $C_{m}$ evolutions for different Reynolds numbers at $\beta=0.5$

The primary interaction stage nominally refers to the range of $-3<x<3$ in this case. In common, approximately at $x=-3$ ("core-in" time), a turning point appears for $C_{x}, C_{y}$, and $C_{m}$, after which $C_{x}$ and $C_{m}$ change to increase while $C_{y}$ changes to decrease. Approximately, at $-3<x<0$, the 
right half of the tornado core moves through the cylinder, followed by the crossing of the rest left half when $0<x<3$. A more detailed look into Fig. 2.12 - Fig. 2.14 can discover that, at $x=0$ ("coinciding-center" time at which the tornado and building centers are nominally considered coinciding), $C_{x}$ and $C_{m}$ are both a bit negative while $C_{y}$ is still positive. This signifies that the two centers are physically not exactly coinciding due to the concurrent rotation and translation ingredients in a tornado scenario. In Fig. 2.12, a second turning point is a bit before $x=-3$ ("core-out" time), after which $C_{x}$ drops again; then, a third turning point is a bit after $x=-3$, after which $C_{x}$ re-rises monotonically. The variation trend of $C_{m}$ shown in Fig. 2.14 looks similar to $C_{x}$ in Fig. 2.12. In Fig. 2.13, the second turning point for $C_{y}$ to monotonically re-rise is approximately located at $x=-3$, without a third turning point detected. By recalling Fig. 2.11, at the "core-out" time, disturbance is obviously seen in the wake region after the tornado core crosses the building through the primary interaction stage, leaving unevenly shed vortices in the wake with the subsequent post-interaction stage.

Although this flow is considered mainly dominated by the translational component because of the relatively low rotation intensity $(\beta=0.5)$, the characteristics reflected by $C_{x}$, for instance, at $R e$ $=1000$, as included in Fig. 2.12, no longer contain any periodicity as shown in Fig. 2.7, in which a purely translational flow at $R e=1000$ was examined. In this sense, the involvement of rotational component can radically change the flow nature regardless of the degree of rotation intensity.

In Fig. 2.12, if taking the extremum $C_{x}$ at the "core-in" time as an observation point for the 
"drag" coefficient, it can be noticed that, at this low rotation intensity $(\beta=0.5)$, the magnitude of this representative $C_{x}$ keeps increasing when $R e$ rises from 1000 to 3900 and, quantitatively, $C_{x}$ goes up by about $16.5 \%$ if comparing this coefficient at $R e=3900$ to $R e=1000$. This increasing trend is also seen for $C_{d}$ in Fig. 2.8 where a purely translational flow was investigated, which confirms that the translation part can overall dominate a tornadic wind at a low rotation intensity. Also, in all Fig. 2.12 - Fig. 2.14, a curve corresponding to a higher Reynolds number appears "thicker" than that for a lower Reynolds number. This thickness is an indication of the perturbation in the tornadic wind phenomena; the higher Reynolds number, the "thicker" curve, and the more perturbation.

2.6.2.2 Test series 2: $r_{c} \omega=V_{t} \quad(\beta=1)$

By doubling the angular velocity, $\omega$, while locking all other parameters, the rotation intensity, $\beta$, gets also doubled. The curves of $C_{x}, C_{y}$, and $C_{m}$ corresponding to $\beta=1$ for Reynolds number ranging from 1000 to 3900 are grouped respectively in Fig. 2.15 - Fig. 2.17, respectively.

The overall trends for the evolution of the three aerodynamic coefficient remain practically unchanged if compared to the case of $\beta=0.5$ (Fig. 2.12 - Fig. 2.14), except that more oscillations are found in the post-interaction stage, indicating the perturbation becomes more intensive when the rotation intensity is increased. In addition, perceptible difference between the two test series arises in terms of the degree of the magnitude of wind loadings at the "core-in" time $(x=-3)$. Using the case with $R e=1000$ as example, $C_{x}$ increases from 2.5 to 9.8 when the rotation intensity changes from 
0.5 to $1.0, C_{y}$ and $C_{m}$ increase nearly doubled. Clearly, the increase of rotation intensity leads to elevated tornado-like wind loadings. This is because $\beta=1$ signifies the magnitudes of the velocity components due to rotation and translation are equivalent, and the shearing stress contributed by the rotation part has become considerable.

In Fig. 2.15 - Fig. 2.17, a detailed look into the tornadic wind loadings at the "core-in" time reveals that the aerodynamic force and moment still monotonically increase when Reynolds number goes up. For $C_{x}$, the growth a bit exceeds $11 \%$ when Re rises from 1000 to 3900 (see Fig. 2.15).

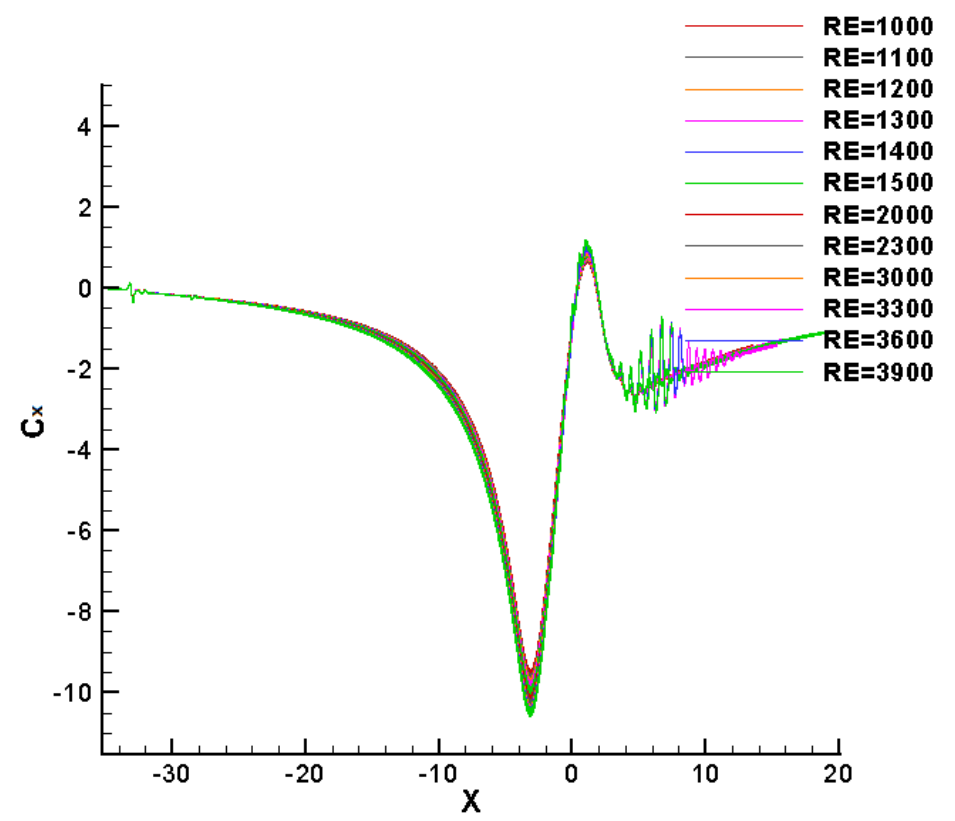

Fig. 2.15 $C_{x}$ evolutions for different Reynolds numbers at $\beta=1$ 


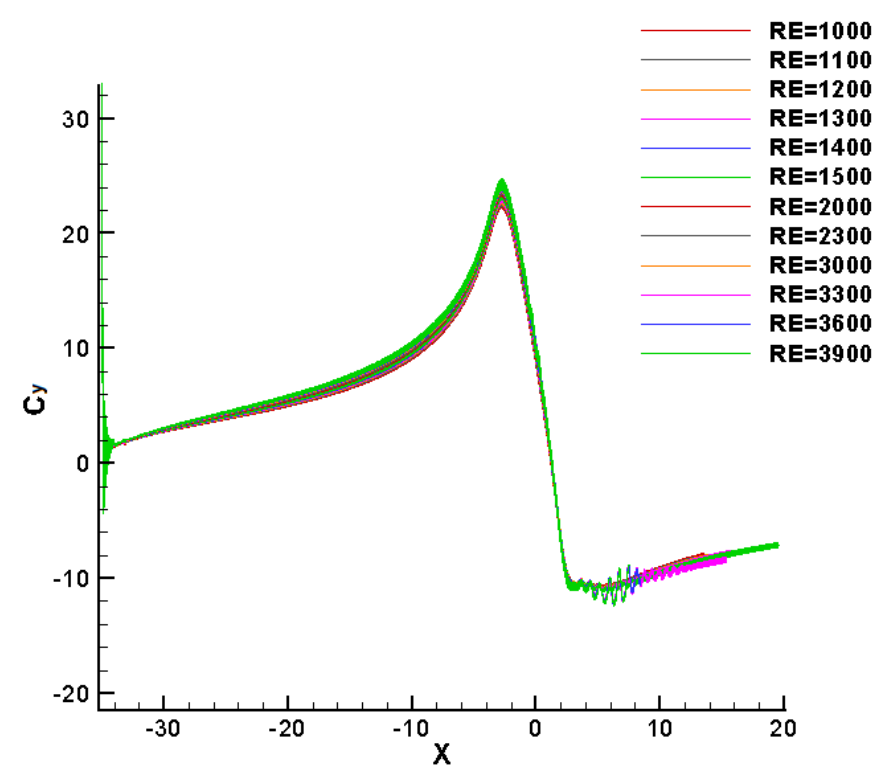

Fig. 2.16 $C_{y}$ evolutions for different Reynolds numbers at $\beta=1$

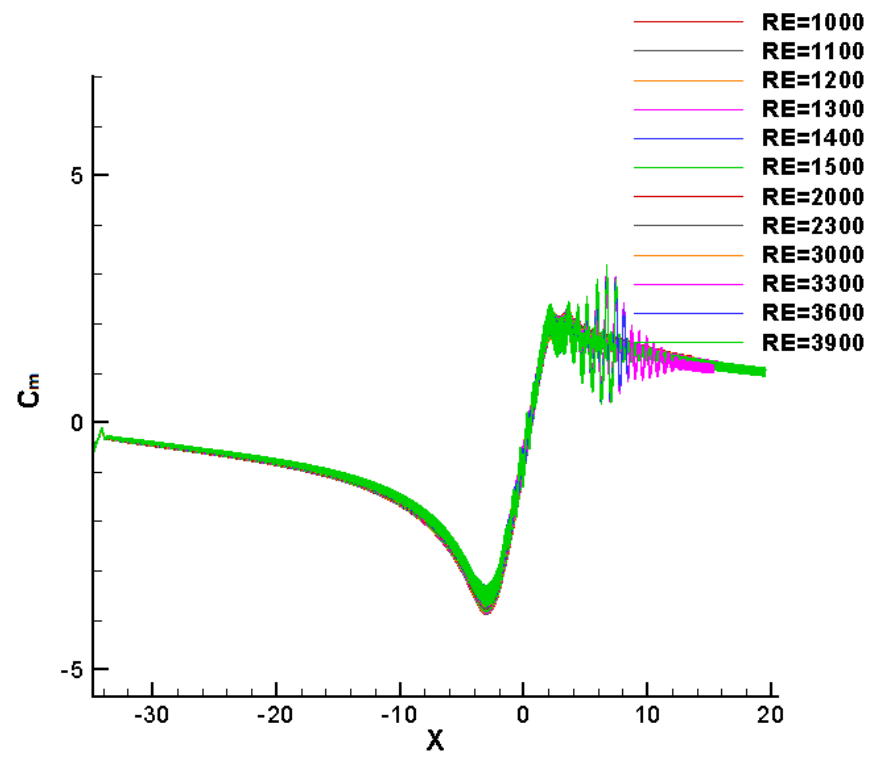

Fig. 2.17 $C_{m}$ evolutions for different Reynolds numbers at $\beta=1$

2.6.2.3 Test series 3: $r_{c} \omega=2 V_{t} \quad(\beta=2)$

When the rotation intensity further increases to 2.0 and up, again, as a representative index of 
tornadic wind loadings, $C_{x}$ at the "core-in" time no longer monotonically goes up with the increase of Reynolds number, as detected in Fig. 2.18 that depicts the case of $\beta=2$ as an example. An enlarged view focusing on this extremum point is shown in Fig. 2.18(b), demonstrating the magnitude of $C_{x}$ reaches its maximum when $R e$ is 3000 although the upper limit of $R e$ is 3900 in this test series.

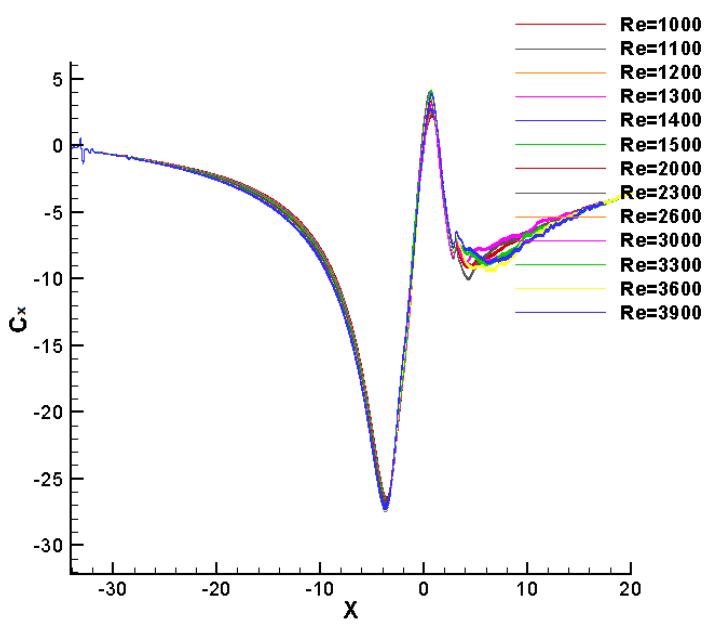

(a)

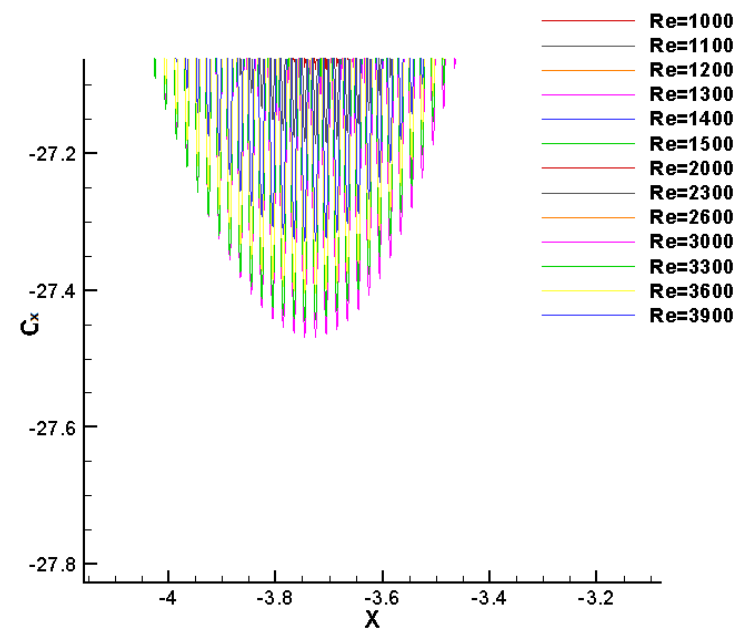

(b)

Fig. $2.18 C_{x}$ evolutions for different Reynolds numbers at $\beta=2$

If comparing Fig. 2.18 to Fig. 2.15 and Fig. 2.12, the overall wind loadings on the obstacle are found much larger than those for $\beta=1$ or less. When looking into the cases with $R e=3900$ but different rotation intensities $\left(\beta=0.5,1,1.5,2,2.5\right.$, and 3), the magnitude of $C_{x}$ at the "core-in" time is found monotonically increasing with $\beta$, as shown in Fig. 2.19. This suggests that, in the study of tornadic wind, the rotation intensity serve as a crucial parameter in addition to the Reynolds number that is determined conventionally using only the incoming translation velocity component as the 
characteristic velocity.

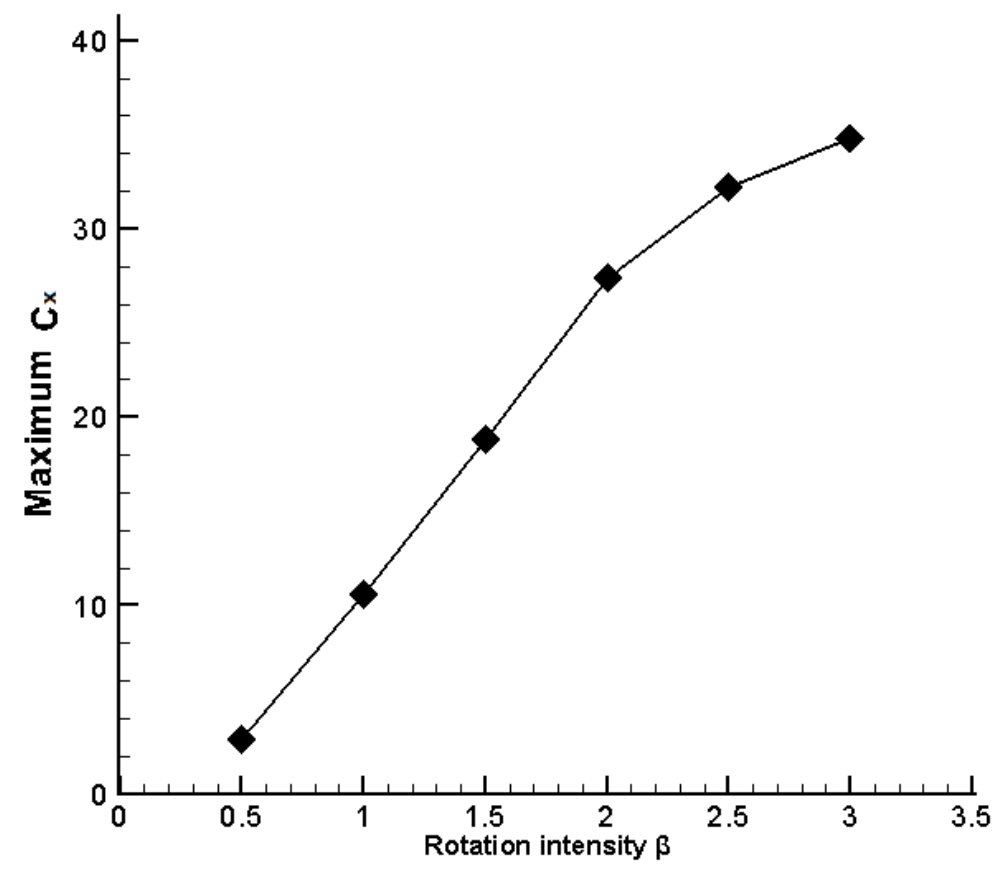

Fig. 2.19 Maximum $C_{x}$ comparison for different $\beta$ at $R e=3900$

\subsubsection{Critical rotation intensity}

Through the test cases conducted in Section 2.6.2 that unfold with different rotation intensities over the same range of Reynolds number (from 1000 to 3900), the distinction appears in terms of the monotonicity of the increase of aerodynamic forces with the increase of $R e$. When the rotation intensity is relatively small, such as $\beta=1$, wind loadings can be regarded as related to Reynolds number only (see, for instance, Fig. 2.15). When the rotation intensity rises to, for example, $\beta=2$, the variation trend for the loadings exerted on the obstacle becomes unpredictable if still relying on $R e$ as the sole influencing factor (see Fig. 2.18). Of practical interest is to locate the critical rotation 
intensity, beyond which the wind loadings no longer monotonically grow up with the increase of Reynolds number. An efficient way to complete this search is to use a strategy that resembles the Newton's bi-section method. That is, given the numerical results obtained in the test series with $\beta$ $=1$ and $\beta=2$, the critical rotation intensity is believed to arise in the interval $[1.0,2.0]$. Then, the new testing rotation intensity is set using:

$$
\beta_{\text {new }}=\frac{1}{2}\left(\beta_{\text {mono }}+\beta_{\text {non-mono }}\right)
$$

for checking the monotonicity in question. After the monotonicity is confirmed at $\beta=1.5, \beta_{\text {mono }}$ is substituted by 1.5 to set a further testing rotation intensity. Employing this bi-section-like strategy, the critical rotation intensity is found to be located within the interval $[1.875,1.9375]$ if the interval length, $\Delta \beta=\frac{1}{16}$, is considered acceptably small. In other words, when the rotation intensity falls lower than the critical interval, the loadings on the cylinder can be viewed as relevant mainly to Reynolds number, and the loading extrema rise with the increase of $R e$; otherwise, no predictable relation is present between the loadings and $R e$. This investigation reveals that, when the rotation intensity is above its critical value, the rotational component of a tornadic wind strongly affects the overall flow characteristics, and the translational velocity component based Reynolds number becomes no longer a sole dominant factor.

\subsection{Conclusions}

In this paper, the classical Rankine-Combined Vortex Model (RCVM) is re-tailored so that an 50 
immersed boundary (IB) lattice Boltzmann method (LBM) can be handily applied to the investigation of the tornado-construction interaction mechanism, and this hybrid framework has also been enriched by the sub-grid stress (SGS) model, so that the simulation of a tornado-building interaction scenario at an elevated Re becomes manageable with ease. The effectiveness of the present IB-LBM computational framework with the re-tailored RCVM embedded is demonstrated through the validating cases in which the IB-LBM results respectively for translational and rotational flows are found in good agreement with the simulation data obtained elsewhere using other numerical approaches.

The focus of this study is mainly placed on the new exploration of the factors that influence the characteristics of a tornadic wind. In fluid dynamics, Reynolds number is usually employed for categorizing viscous fluid flow. Particularly, when studying the tornado-like flows, the practitioners have been conventionally using the translational velocity component in RCVM as the characteristic velocity for determining the Reynolds number, and considering such a translation component based Reynolds number as a primary influencing parameter. The present study has found that the translation part based Reynolds number is of one-sided nature for identifying a tornado-like flow, since the rotation is the other part of a tornado and the change of the rotation intensity, as defined in Eq. (2.4), may strongly alter the tornado characteristics.

In order to evaluate the impact of the rotation intensity, the test scenario employed in this study is that a tornado-like wind passes over a cylinder. In a number of test series included in this paper, 
the investigation has been performed by checking, for a given rotation intensity, whether the aerodynamic coefficients monotonically rise with the increase of Reynolds number. It is found that this monotonicity holds at lower rotation intensities while disappearing at elevated rotation intensities. Then, for the purpose of capturing the critical rotation intensity that demarcates the two intervals, one being practically considered as solely dominated by Reynolds number and the other no longer manifesting that manner, a Newton's bi-section-like strategy is presented in this study, and this critical value has been detected to be within the range from 1.875 to 1.9375 in the test scenario reported in this paper.

In practice, when the rotation intensity is less than its critical value, the translational flow component can be considered dominant in the tornado dynamics, and this falls in the conventional understanding of the tornado investigation community. Many previously achieved findings in the tornado study fell into this interval, but would not necessarily apply to the other interval in which the critical rotation intensity has been exceeded. This study tends to draw more attention of tornado researchers to the tornado cases with rotation intensities greater than the critical value in spite of more challenges and complexity in this veiled tornado research branch, where the rotation part is of the same or even higher degree of significance, if compared to the translation part, and the disasters incurred are often more dreadful. 


\section{Chapter 3 A two-dimensional IB-LBM framework combined with re-tailored RCVM for assessing the rotation intensity of a tornadic wind over a building configuration}

This chapter is based on the following published paper:

Xixiong Guo, Rangaraj Palanisamy, Jun Cao. A two-dimensional IB-LBM framework combined with re-tailored RCVM for assessing the rotation intensity of a tornadic wind over a building configuration. Engineering Structures, 131, 57-68, 2017.

\subsection{Summary}

Following the study in Section 2.6, the present IB-LBM simulations are aimed to investigate the tornado-like wind effects on a building configuration in different orientations and, particularly, seek the relation between the rotation intensity of a tornado and the wind loadings on the constructions. Through comparison at a series of rotation intensities, the loading extreme value is observed to be unrelated to Reynolds number once the rotation intensity exceeds a critical value, which is similar to the conclusions drawn in Chapter 2. These simulation results reveal that it looks inappropriate to rely solely on the translational velocity component to characterize tornadoes, and call on additional attention towards the rotation intensity for a more comprehensive tornado dynamics study. 
It is noticed that the reported computational analysis of tornado dynamics has placed more emphasis on the translational component since the translational velocity is conventionally chosen to serve as the characteristic velocity for the Reynolds number determination. Physically, both the translational and rotational components play simultaneous roles in a tornado wind field. The evaluation of the overall tornado wind loading without due consideration of the influence of the rotational component would turn out to be inaccurate and unreliable, possibly leading a structure design to miscalculated wind-resistant capabilities; however, the relation between the tornado wind load and its rotational component has been seldom reported in existing literature. In order to gain a better understanding in this regard, this study aims at the simulation of tornado-like winds at different rotation intensities over a building configuration with orientation deployed, while the focus is placed on a parametric study for each of the tested orientations towards capturing a critical rotation intensity that discontinues the monotonic relation between the increases of tornadic wind loading and Reynolds number. Meanwhile, this study aims to scrutinize the correlation between the aerodynamic loads and the rotational component of a tornadic wind by means of numerical simulation.

\subsection{Tornado-like flow model and numerical method}

The Rankine-combined vortex model (RCVM) introduceed in Section 2.3.1 is employed to describe the tornado in this work. Furthermore, with the aid of "relative motion" principle, the 
customized RCVM (see Section 2.3.2) is also adopted, which reinterprets the scenario of a tornado towards building configuration as a "virtual" translation of building towards a rotating airflow about its "locked" center. As illustrated in Fig. 3.1, this new representation greatly facilitates the establishment of boundary condition owing to its time-independent feature when simulating a bi-cylinder building configuration. IB-LBM framework is employed in this tornado simulation, and details have been illustrated in Section 2.4.

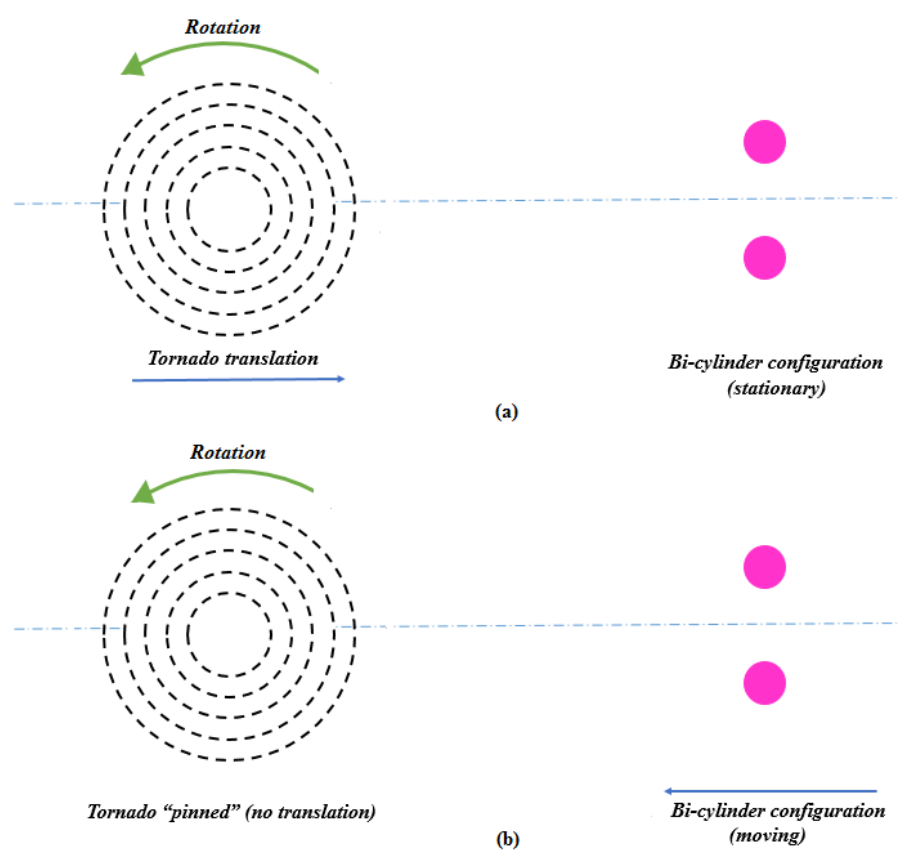

Fig. 3.1 Physical and re-tailored RCVM (a) physical tornado model; and (b) re-tailored tornado model.

\subsection{Validation}

As aforementioned, very limited literature about numerical simulation of tornado-induced wind load on buildings can be found; in particular, those using RCVM as model basis are even fewer. 
Thus, other comparable cases need to be sought to validate the present approach. In the previous chapter, this proposed IB-LBM framework has been validated through translational flow at elevated Reynolds number, and rotational Couette flow cases. Moreover, another case of a flow induced by a counter-clockwisely rotationally oscillating cylinder, as numerically simulated in [51]-[52], is of some relevant interest to tornado simulation, since that case also involves the fluid dynamics due to fairly complex rotation. The rotationally oscillating cylinder induced flow simulation is hence chosen to test the present IB-LBM framework and, particularly, its capability of simulating the rotational flow evolution.

The motion of rotationally oscillating cylinder is prescribed by the following time-dependent angular velocity:

$$
\gamma(t)=\gamma_{0} \sin (2 \pi f t)=A \frac{2 V_{t}}{D} \sin \left(2 \pi S t \frac{V_{t}}{D} t\right)
$$

where $f$ and $\gamma_{0}$ are the frequency and rotation amplitude, respectively. These two parameters can be normalized by introducing two non-dimensional parameters: Strouhal number $S t=\frac{f D}{V_{t}}$ and normalized rotation amplitude $A=\frac{\gamma_{0} D}{2 V_{t}}$. This validation case employs $A=2.0, \mathrm{Re}=100$, and $S t=0.163$ in accordance with the available references [51]-[52]. As illustrated in Fig. 3.2, the instantaneous vorticity field obtained using IB-LBM matches well with the results of Wu et al. [51] and Choi et al [52]. The vortex shedding phenomenon can be clearly observed using the present method, and the pattern of the vortices released from the obstacle looks overall the same between 
each other.

The validation can be also performed using the time-averaged drag coefficient, $\overline{C_{d}}$, and the maximum amplitude of the lift coefficient fluctuation, $C_{l}^{\prime}$, leading to a quantitative comparison against existing references [51]-[52] as depicted in Table 3.1. Here, $S t=0.4$ is taken as [51]-[52] examined this Strouhal number for evaluation of these two parameters; other parameters remain the same as aforementioned. Both $\overline{C_{d}}$ and $C_{l}^{\prime}$ obtained in this study are found comparable with the results reported in [51]-[52], as demonstrated in Table 3.1. The small differences may be attributed to the computational domain and meshing arrangement that were employed differently in the three simulation sets of this benchmark case. Through comparisons of the vorticity contour and aerodynamic parameters, the present IB-LBM framework demonstrates its reliability for complex rotational flow simulation. Besides, the grid independence study corresponding to the IB-LBM framework was conducted previously [49], revealing that this integrated numerical approach has approximately a second order of accuracy, and the results can be considered as grid-independent when the grid resolution is finer than $1 / 40$, i.e., the length of cylinder diameter should be uniformly divided into 40 or more equal portions when discretizing the computational domain. The present tornado study employs this numerical framework with a grid resolution of $1 / 50$, and it is reasonable to believe the resulting simulation is reliable. 


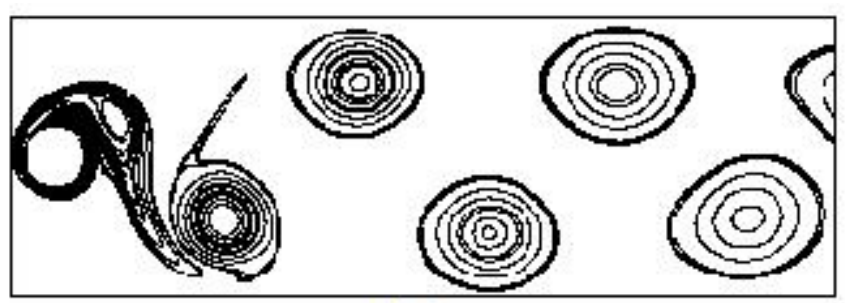

(a)

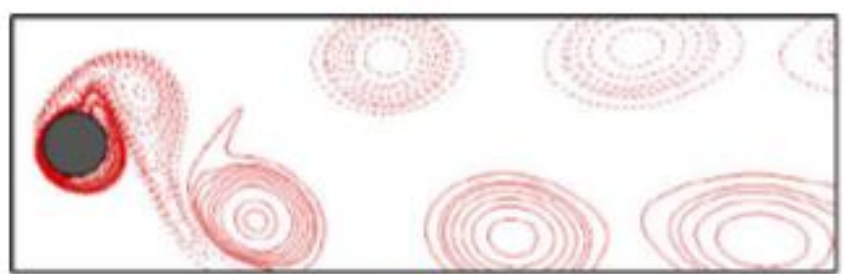

(b)

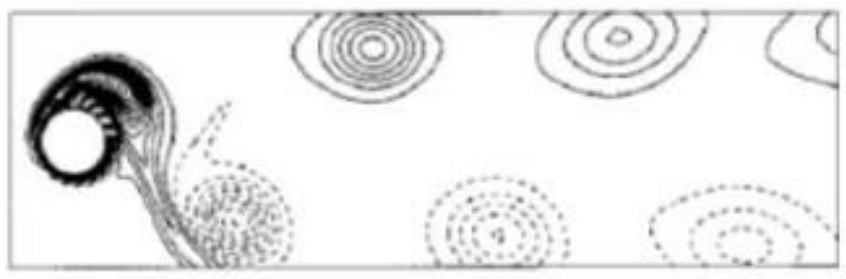

(c)

Fig. 3.2 Vorticity contour results from (a) the present method; (b) Wu et al. [51]; (c) Choi et al. [52]

Table 3.1 Comparison about drag and lift coefficients at $R e=100$

\begin{tabular}{ccc}
\hline References & Drag coefficient $\overline{C_{d}}$ & Lift coefficient $C_{l}^{\prime}$ \\
\hline Wu et al. [51] & 1.302 & 0.321 \\
Choi et al. [52] & 1.231 & 0.299 \\
Present & 1.286 & 0.320 \\
\hline
\end{tabular}

\subsection{Numerical experiments}

The stylized building configuration for test use in this study includes two identical cylinders of 
diameter $D$ that are gapped by $3 D$ and oriented in three manners facing the incoming translation of a tornadic wind: vertical, horizontal, and $45^{\circ}$-inclinedall. The tornado and domain settings for this work keep the same as Section 2.6. Using the re-tailored RCVM, the center of the counterclockwise rotational flow component is fixed at $(0,0)$, and initially the building configuration center is placed at $(35,0)$ with a constant translational speed towards the negative $x$-direction, as physically the tornado is translating to the positive $x$-direction.

For each orientation, the testing Reynolds number ranges from 1000 to 3500 through adjusting the fluid viscosity while locking the characteristic velocity, $V_{t}$, and length, $D$. Three loading coefficients, $C_{x}, C_{y}$, and $C_{m}$, which reflect the aerodynamic force components in the $x$ - and $y$-directions, and the resulting moment, $M$, respectively (Eqs. (2.41)-(2.43)) serve to examine the wind loading on the cylinders. This study will evaluate the relation between Re and the aerodynamic force, i.e., wind loading, exerted on bi-cylinder configurations in tornadoes with different rotation intensities, $\beta$, aiming to capture the critical rotation intensity from which the tornado characteristics deviation arises.

\subsubsection{Vertically arranged bi-cylinder}

First, two vertically arranged cylinders are tested. Under rotation intensity $\beta=1.5$, the simulation results of the streamline pattern with $R e=1000$ at the "coinciding-center" time, along with the $C_{x}, C_{y}$, and $C_{m}$ evolutions at different testing Reynolds numbers are respectively plotted in 
Fig. 3.3 - Fig. 3.6. Note the horizontal coordinate, X, in Fig. 3.4 - Fig. 3.6 is arranged such that, physically, while $X<0, X=0$, and $X>0$, the tornado center approaches the construction, coincides with the construction center in the nominal sense, and leaves the construction, respectively.

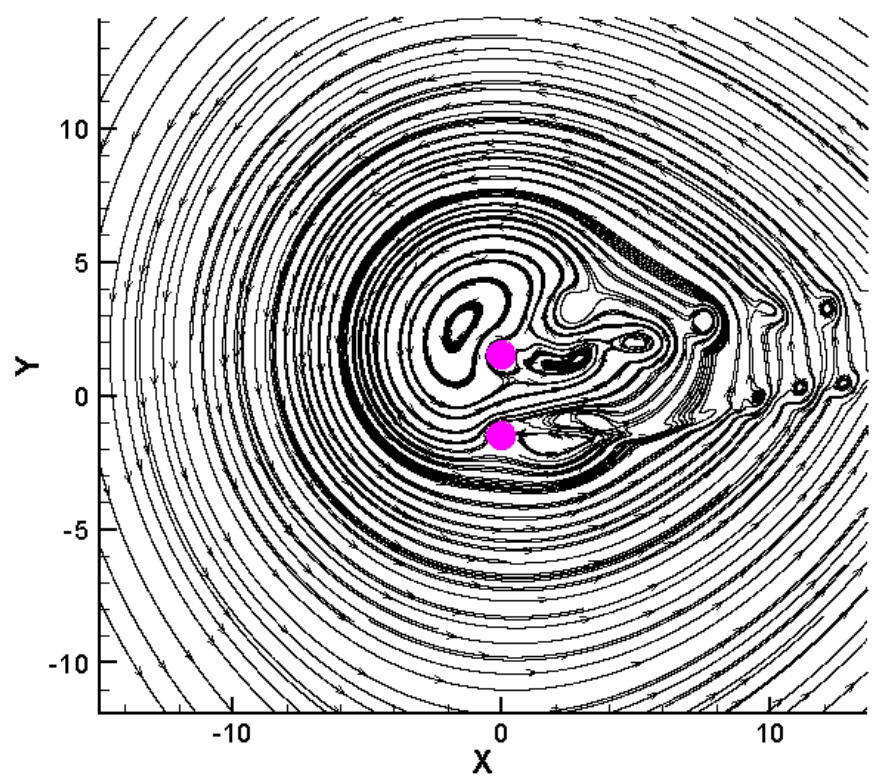

Fig. 3.3 Streamlines at "coinciding-center" time for vertically arranged bi-cylinder streamline at $\operatorname{Re}=1000$ and $\beta=1.5$.

When the translating tornado center approaches the bi-cylinder construction, Fig. 3.3 demonstrates that the tornado core area is strongly disturbed with two series of eddies found in the wake region, suggesting the local dominance of the translational flow component upon recalling the renowned Karman vortex street phenomenon that arises in the case of a single cylinder facing a purely translational incoming flow with an elevated Reynolds number. On the other hand, due to the elapsing tornado-construction interaction, at the theoretical "coinciding-center" time, Fig. 3.3 does 
not illustrate the tornado and construction centers are exactly coinciding; instead, a slight upward trend is clearly seen for the flow in the wake region, which is attributed to the counter-clockwise rotational flow component in this tornadic wind. All observed characteristics collectively reflect the translation and rotation superposition nature of this tornadic wind. Consequently, the loadings $C_{x}, C_{y}$, and $C_{m}$ on the upper cylinder can be observed apparently higher than those on lower cylinder, as shown in Fig. 3.4 - Fig. 3.6; also, the loading coefficients in both directions can be regarded as related with Reynolds number at $\beta=1.5$, since the extreme points of $C_{x}, C_{y}$, and $C_{m}$ monotonically increase with the Reynolds number.

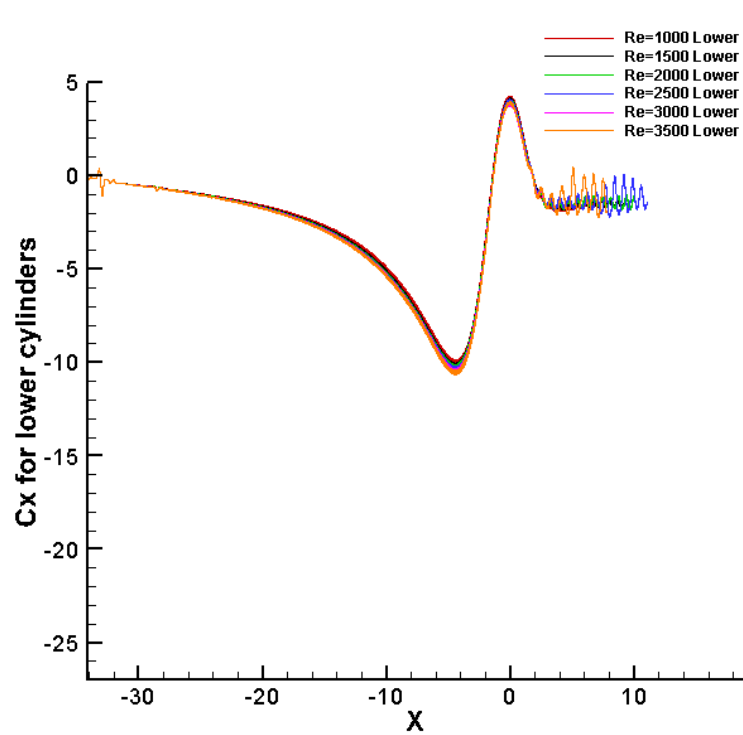

(a)

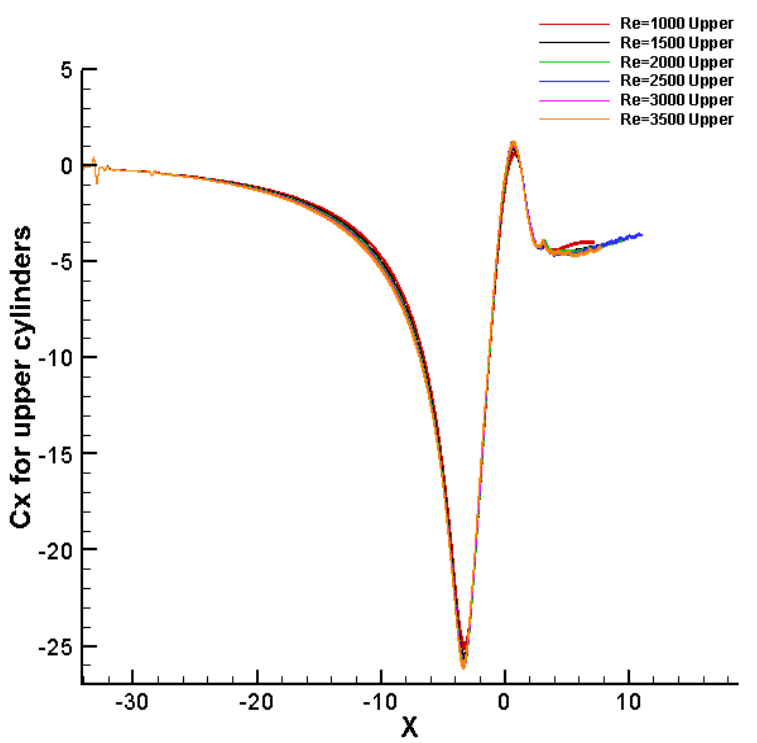

(b)

Fig. 3.4 $C_{x}$ evolutions for vertically arranged bi-cylinder at $\beta=1.5$, under different $R e$

(a) lower cylinder; (b) upper cylinder. 


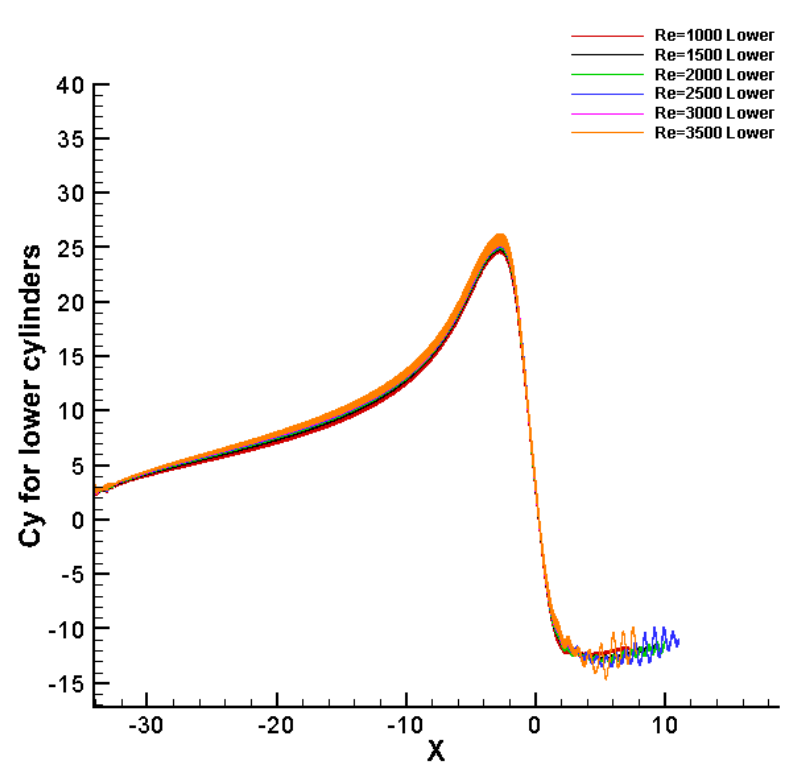

(a)

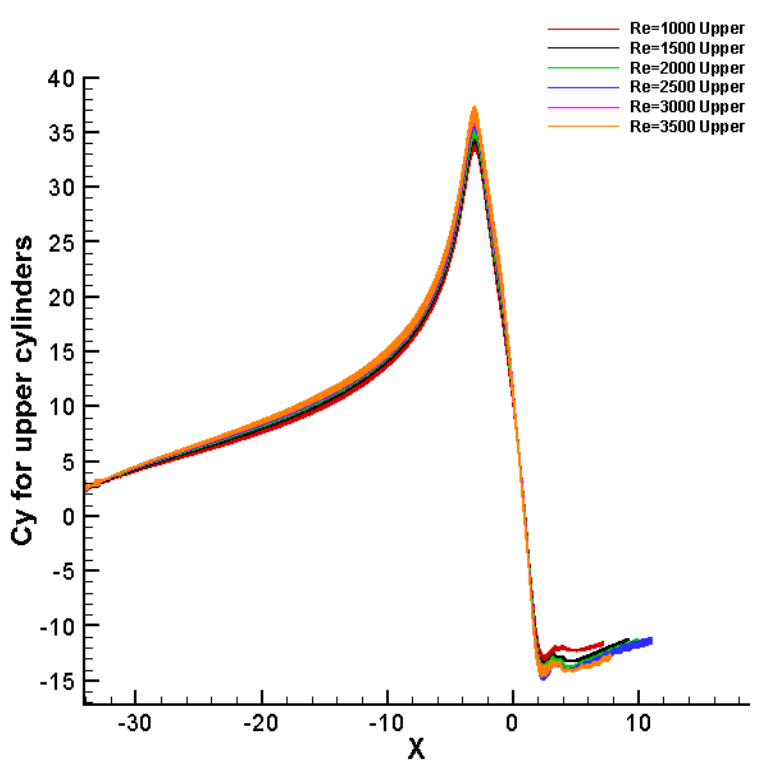

(b)

Fig. 3.5 $C_{y}$ evolutions for vertically arranged bi-cylinder at $\beta=1.5$, under different $R e$

(a) lower cylinder; (b) upper cylinder.

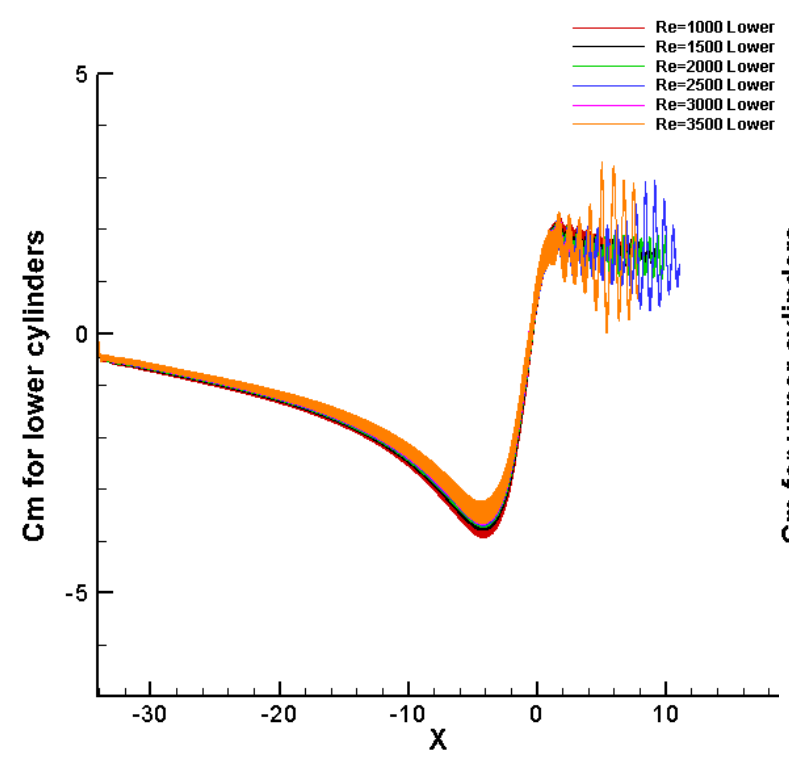

(a)

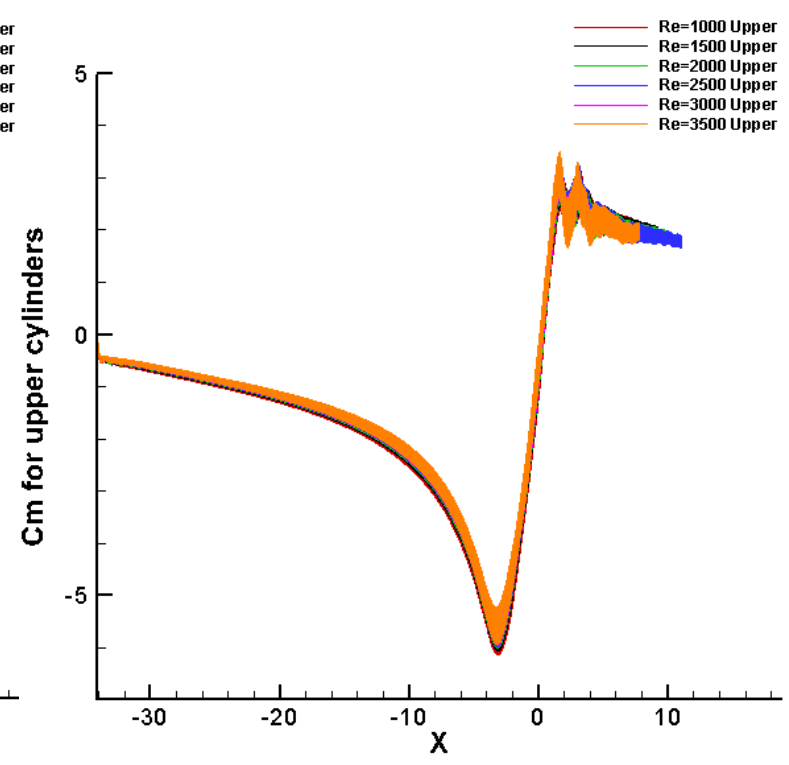

(b)

Fig. 3.6 $C_{m}$ evolutions for vertically arranged bi-cylinder at $\beta=1.5$, under different $R e$ (a) lower cylinder; (b) upper cylinder.

However, the monotonicity between loading coefficients and Reynolds number is not persistent 
at all rotation intensities in the tornado case. When a more intense rotational component is present, such as $\beta=2$, the extreme point of $C_{x}$ at the "core-in" time no longer monotonically goes up with the increase of Reynolds number, as detected in Fig. 3.7 and further confirmed by an enlarged view in Fig. 3.8. Remark that the magnitude of $C_{x}$ reaches its maximum when $R e$ is 3000 although the upper limit of $R e$ is 3500 in this test series. This suggests that, in the tornado dynamics study, the rotation intensity serves as a crucial parameter in addition to the Reynolds number that remains conventionally determined using only the incoming translation velocity component as the characteristic velocity.

By further testing different rotation intensities over the same range of Reynolds number (from 1000 to 3500 ), the "critical rotation intensity" is sought that discontinues the monotonicity of the aerodynamic forces with the increase of $R e$. A simple and yet efficient way to perform this search is to use a Newton's bi-section strategy. That is, given the numerical results corresponding to $\beta=1.5$ and $\beta=2$, the critical rotation intensity is believed to arise in the interval $[1.5,2.0]$. Then, the new testing rotation intensity is set using:

$$
\beta_{\text {new }}=\frac{1}{2}\left(\beta_{\text {mono }}+\beta_{\text {non-mono }}\right)
$$

for checking the monotonicity in question. After the monotonicity is confirmed at $\beta=1.75, \beta_{\text {mono }}$ is substituted by 1.75 to set a further testing rotation intensity. Employing this bi-section method, the critical rotation intensity is found to be located within the interval [1.78125, 1.8125], where $\Delta \beta=1 / 32$ can be referred to as "critical interval" if this interval length is considered acceptably 
small. In other words, when the rotation intensity falls the left side of the critical interval, the wind loadings can be viewed as relevant mainly to Reynolds number, and the loading extreme point rises with the increase of $R e$; otherwise, there is no predictable relation between the loadings and $R e$. Hence, when the rotation intensity is above its critical value, the rotational component of a tornadic wind strongly affects the overall flow characteristics, and the translational velocity component based Reynolds number is no longer the sole dominant factor.

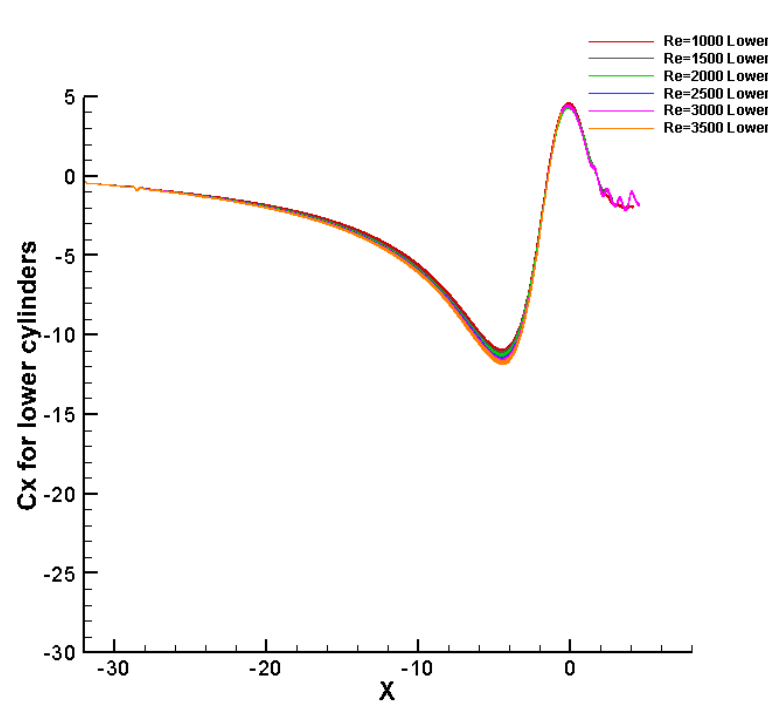

(a)

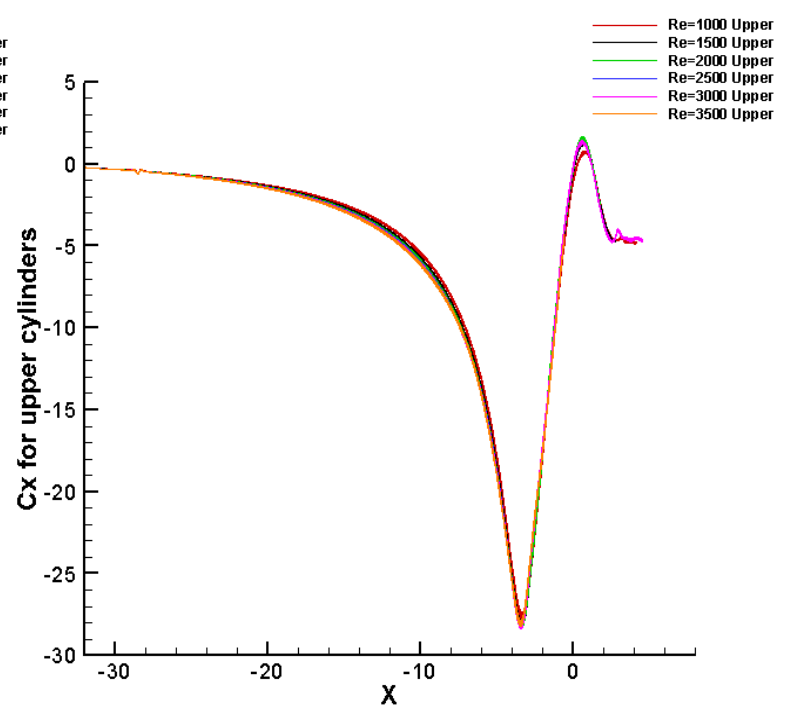

(b)

Fig. 3.7 $C_{x}$ evolutions for vertically arranged bi-cylinder at $\beta=2$, under different $R e$

(a) lower cylinder; (b) upper cylinder. 


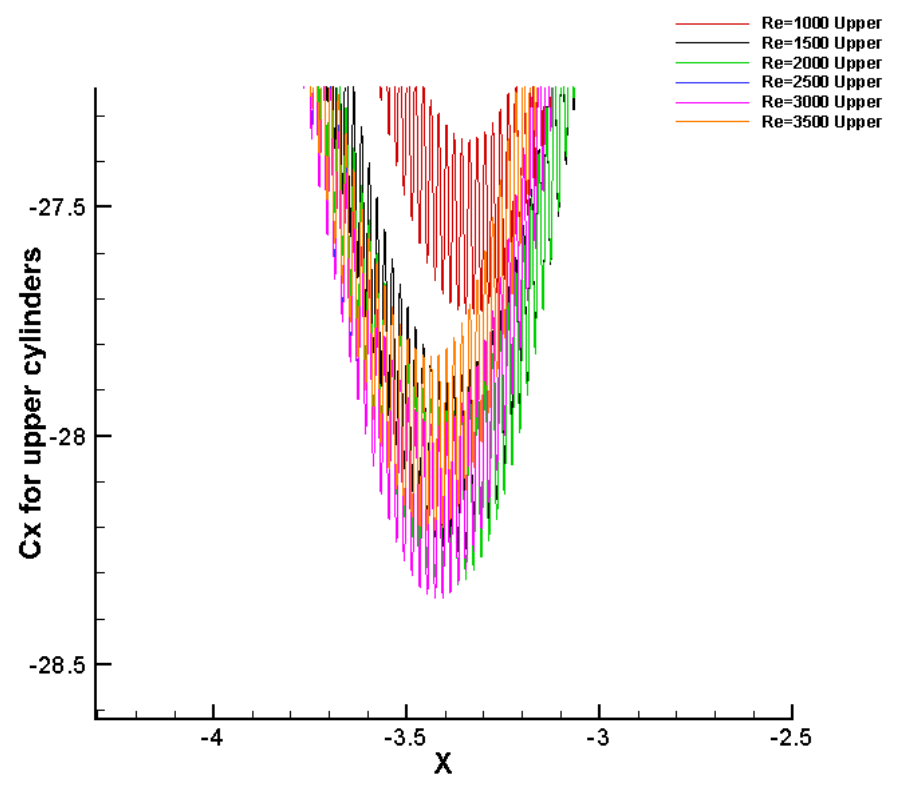

Fig. 3.8 $C_{x}$ extreme points (for the upper cylinder) for vertically arranged bi-cylinder at $\beta=2$, under different $R e$.

\subsubsection{Horizontally arranged bi-cylinder}

Now the two cylinders are arranged horizontally, the tornadic wind streamlines at $R e=1000$ and $\beta=1$ are plotted, in Fig. 3.9, around the construction at the "coinciding-center" time. Unlike in the precedent vertically arranged bi-cylinder case, mainly only one Karman vortex street is manifested in the wake; again, this vortex street still appears slightly upward due to the counter-clockwsie rotational component in the tornado.

The evolutions of force coefficients for both cylinders in Fig. 3.10 and Fig. 3.11indicate that the trend of the front cylinder overall basically matches with that in the upper one of the previous vertically arranged bi-cylinder; however, the force evolution for the rear cylinder here is observed to be in chaos. Facing the incoming hybrid flow, the front cylinder serves as a shelter to the rear 
cylinder, yielding a strong disturbance to the flow within the gap of the two cylinders and, hence, making it more difficult to predict the loading features for the rear cylinder. Despite the disordered loading evolution for the rear cylinder, the extreme value of loading force measured on the front one at different Reynolds numbers still keep a monotonous trend as long as the rotation intensity is lower than its critical value. The critical intensity is determined within the interval [1.09375, 1.125] (with $\Delta \beta=1 / 32$ as critical interval again) by using the Newton's bi-section method again. Similar to the precedent case, when the rotation intensity $\beta$ is higher than the critical value, Reynolds number is no longer the only dominant factor when the maximum value of force coefficient on the front cylinder is under investigation, as a matter of fact, both rotation intensity and $R e$ shall co-play in such scenarios.

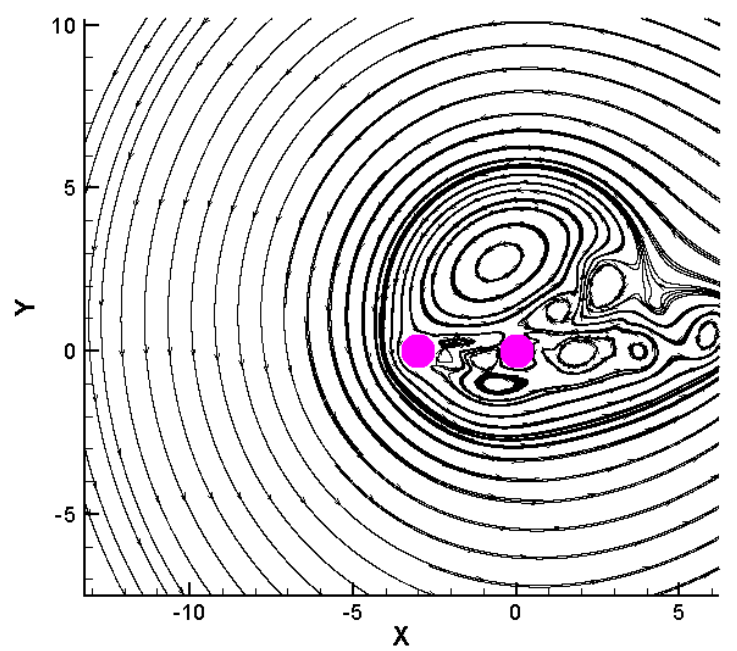

Fig. 3.9 Streamlines at "coinciding-center" time for horizontally arranged bi-cylinder streamline at $R e=1000$ and $\beta=1$. 


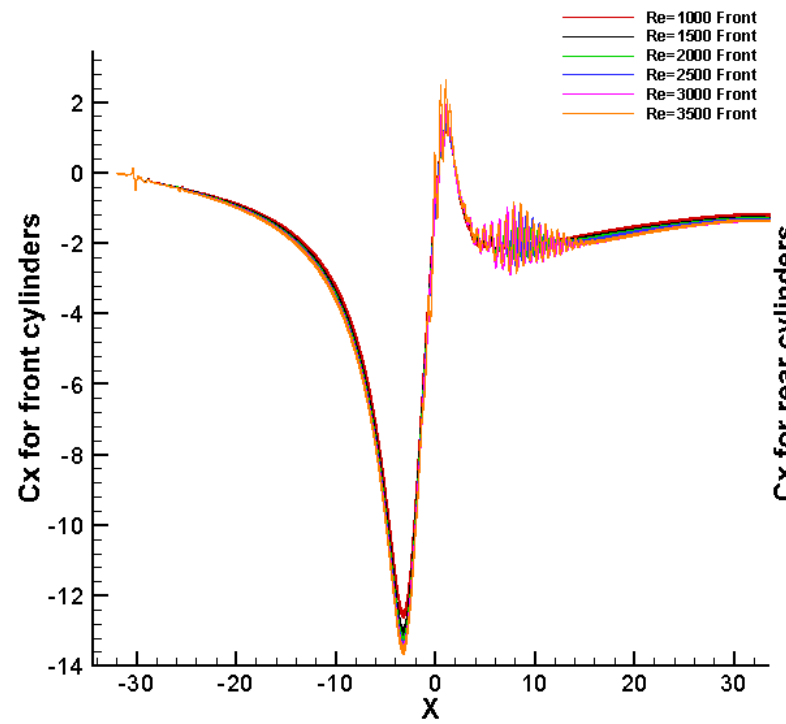

(a)

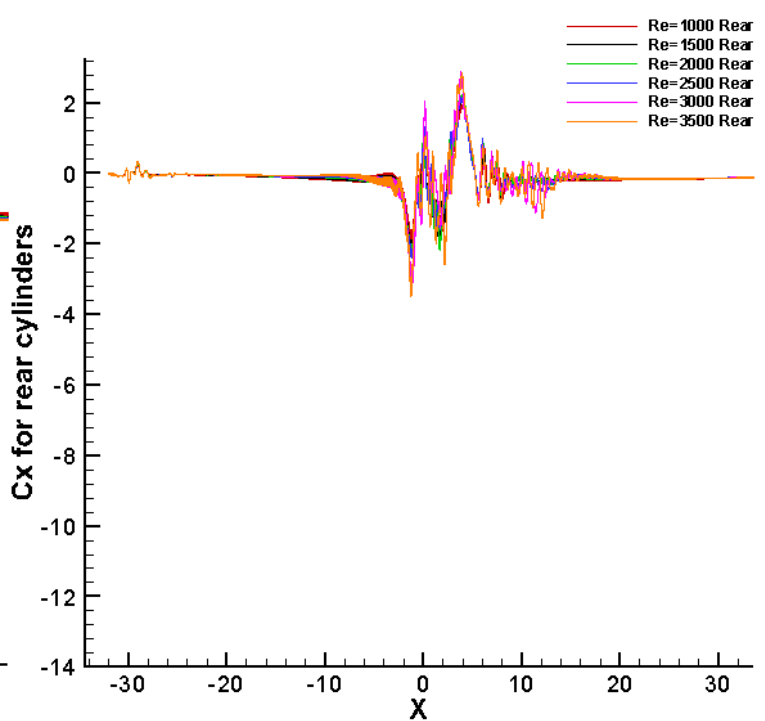

(b)

Fig. 3.10 $C_{x}$ evolutions for horizontally arranged bi-cylinder at $\beta=1$, under different $R e$

(a) front cylinder; (b) rear cylinder.

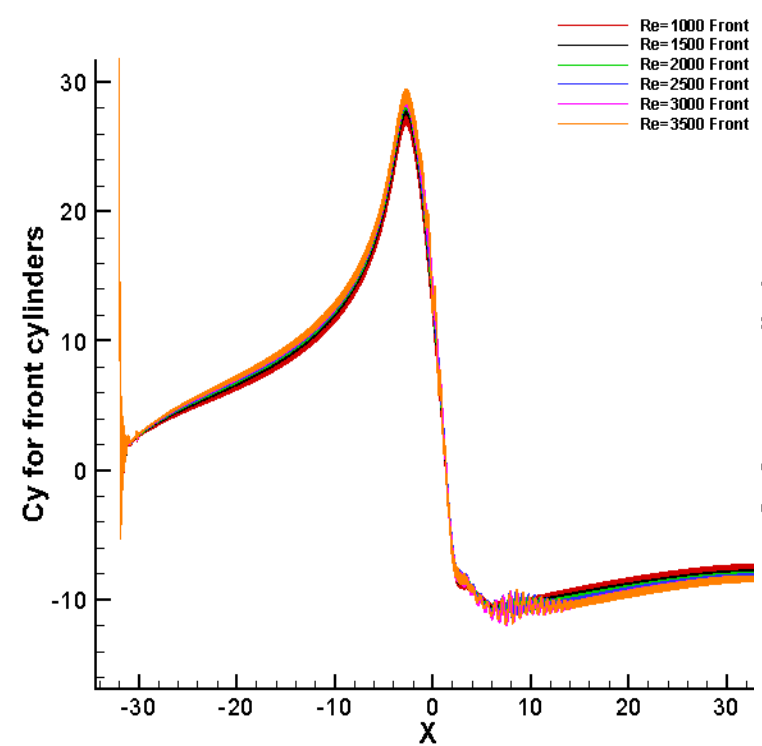

(a)

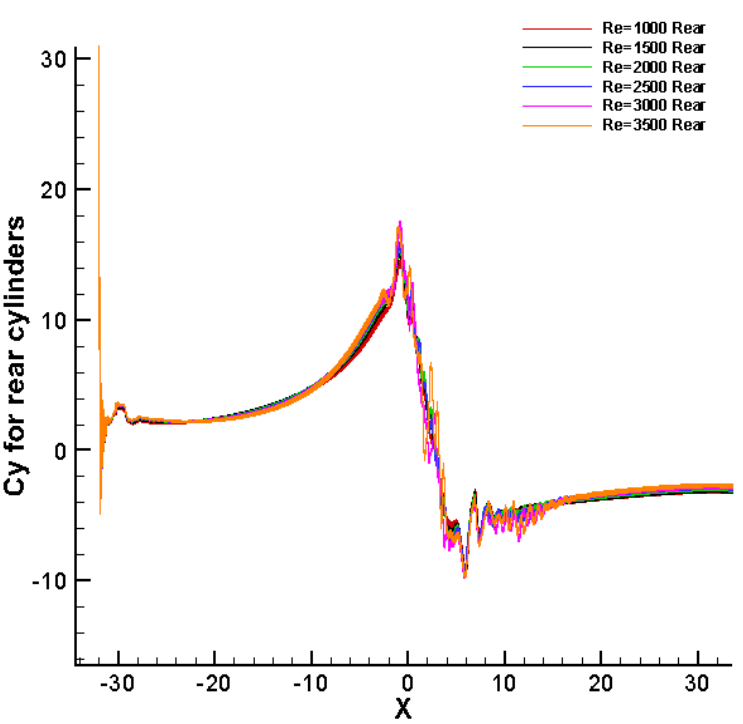

(b)

Fig. 3.11 $C_{y}$ evolutions for horizontally arranged bi-cylinder at $\beta=1$, under different $R e$ (a) front cylinder; (b) rear cylinder. 


\subsubsection{Inclined bi-cylinder}

When two cylinders are placed to incline at $45^{\circ}$ with respect to the horizontal to sustain a tornadic flow at $R e=1000$ and $\beta=1.375$, the indication of two series of shedding vortices, as shown in Fig. 3.12, is stronger than in the horizontally placed bi-cylinder case (see Fig. 3.9), but much weaker than in vertically placed bi-cylinder case (see Fig. 3.3). Similar to the case of bi-cylinder horizontal layout, the $C_{x}$ on the front cylinder is observed to be higher than on the rear one, as seen in Fig. 3.13 and Fig. 3.14. Obviously, since physically the tornado core area reaches the front cylinder earlier than the rear one, the overall impulse provided by the aerodynamic forces would be bigger for the front cylinder. Again, of interest in this test case is to detect the critical rotation intensity by using the Newton's bi-section strategy. Here, the tests range for $\beta$ from 0.5 up to 2, ending up with the critical value determined to fall within [1.46875, 1.50] (with $\Delta \beta=1 / 32$ ).

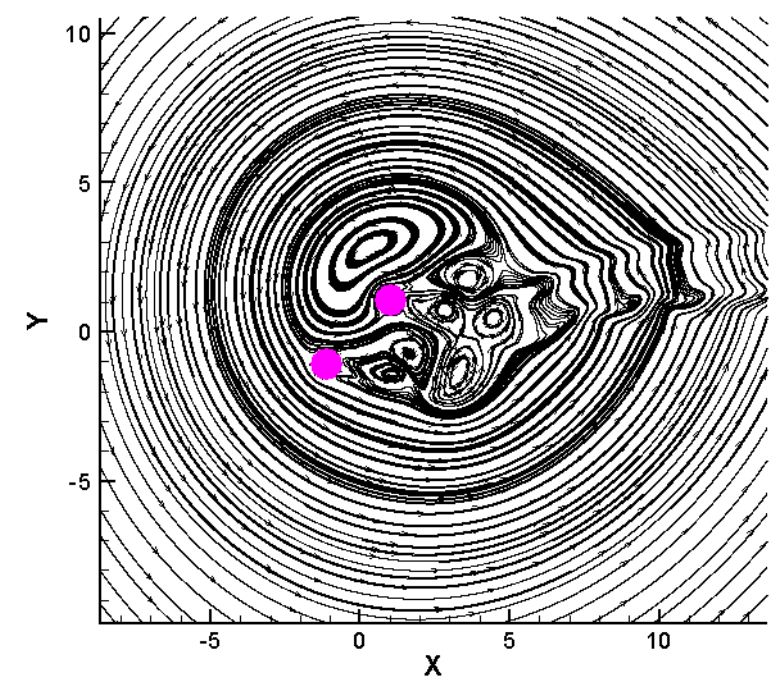

Fig. 3.12 Streamlines at "coinciding-center" time for inclined bi-cylinder streamline at $R e=1000$ and $\beta=1.375$ 


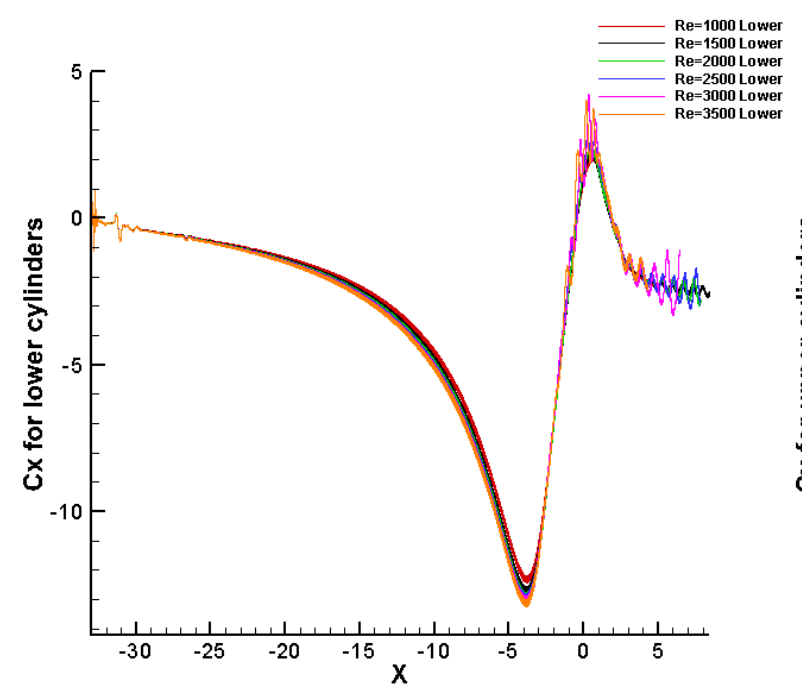

(a)

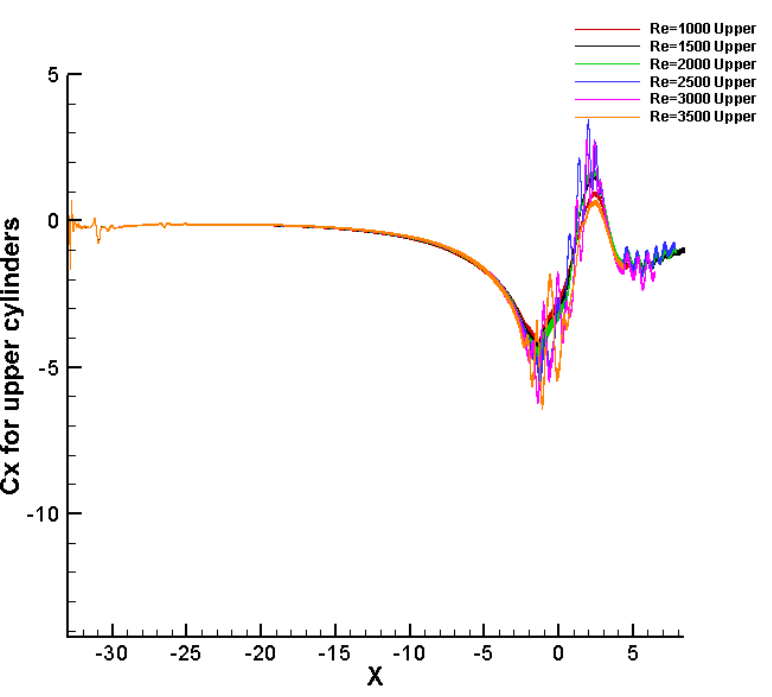

(b)

Fig. 3.13 $C_{x}$ evolutions for inclined bi-cylinder at $\beta=1.375$, under different $R e$

(a) lower cylinder; (b) upper cylinder.

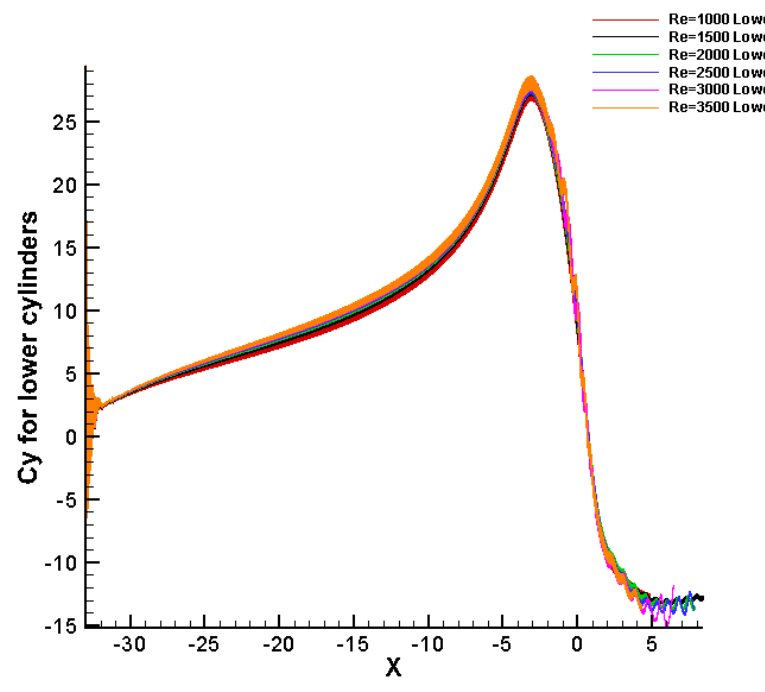

(a)

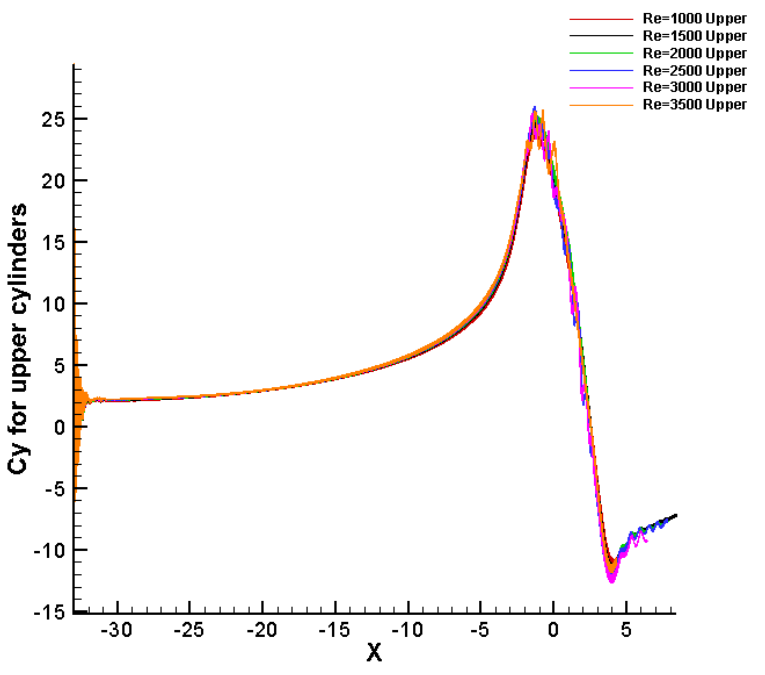

(b)

Fig. 3.14 $C_{y}$ evolutions for inclined bi-cylinder at $\beta=1.375$, under different $R e$

(a) lower cylinder; (b) upper cylinder.

\subsection{Conclusions}

In this study, the conventional Rankine-combined Vortex Model (RCVM) is revised to 
investigate the vortex-structure interaction through immersed boundary (IB) lattice Boltzmann method (LBM). The efficacy of the present IB-LBM framework with the customized RCVM is demonstrated through a validating case in which the vortex shedding pattern behind a rotationally oscillating cylinder is clearly seen and is corroborated with the references.

Numerous factors may affect the characteristics of a tornado-like wind. This study mainly concentrates on the relation between the rotation component and the acuteness of the tornado. The testing geometry is a configuration formed by two gapped cylinders of identical size. Three different orientations are investigated to simulate a tornado translating towards the bi-cylinder construction at attacking angles of $90^{\circ}$ (Section 3.4.1), $0^{\circ}$ (Section 3.4.2), and $45^{\circ}$ (Section 3.4.3). Two terminologies are employed for evaluating the impact of the rotation intensity. The "critical rotation intensity" is defined such that the wind loadings monotonically rise with the increase of Reynolds number when the rotation intensity is lower than its critical value, and this monotonicity would disappear once the critical rotation intensity is reached or exceeded. To numerically detect this critical rotation intensity, the Newton's bi-section approach is proposed and, in practice, an acceptably small "critical interval" for accommodating the critical rotation intensity should be prescribed. Using $\Delta \beta=1 / 32$ as critical interval for the three cases as aforementioned, the characteristic data are grouped in Table 3.2.

The second column of Table 3.2 reveals that, when the tornado translates at nil attack angle towards the bi-cylinder configuration, the critical rotation intensity is the smallest in the three 
orientations. At this orientation, a tornado even with stronger rotational component might not severely damage the construction, which is also justified by the third column (showing the largest $C_{x}$ magnitude) of the table. However, the fourth column (showing the ratio of the bigger $C_{x}$ extreme to the smaller) of the table warns that the front cylinder in the configuration would experience significantly larger wind loadings than the rear one, and the ratio appears to be the largest in the case of nil attacking angle.

Table 3.2 Summarized results for three tested orientations

\begin{tabular}{|r|r|r|c|}
\hline Attack & $\begin{array}{r}\text { Critical rotation } \\
\text { angle }\end{array}$ & Largest & Ratio of the bigger $C_{x}$ extreme to the \\
\hline $0^{\circ}$ & {$[1.09375,1.125]$} & 13.6 & 6.6 (Fig. 3.10, for $\operatorname{Re}=1000, \beta=1)$ \\
\hline $45^{\circ}$ & {$[1.46875,1.5]$} & 14.2 & 3.3 (Fig. 3.13, for $\operatorname{Re}=1000, \beta=1.375)$ \\
\hline $90^{\circ}$ & {$[1.78125,1.8125]$} & 26.4 & 2.5 (Fig. 3.4, for $\operatorname{Re}=1000, \beta=1.5)$ \\
\hline
\end{tabular}

Another remark resulting from this study refers to the role of the rotation intensity in the tornado dynamics study. In tornado-like flows, the customary way of defining the Reynolds number with the translational velocity component as the characteristic velocity looks inequitable and equivocal, since the rotational velocity component plays an equally important role in determining the characteristics of tornadoes as well as imparting the annihilating potential to the tornado. At 
present, the critical rotation intensity is recommended as a complementary parameter for a more comprehensive study on the tornado dynamics. 


\section{Chapter 4 An Immersed-Boundary (IB)-Based Tornado Model for Computational Analysis of Disastrous Wind Load on Buildings - Investigation of Tornadic Wind Load over Cylinder-Quintuplet}

This chapter is based on the following paper (Submitted to Journal of Wind Engineering \& Industrial Aerodynamics): Xixiong Guo, Jun Cao. An Immersed-Boundary (IB)-Based Tornado Model for Computational Analysis of Disastrous Wind Load on Buildings - Investigation of Tornadic Wind Load over Cylinder-Quintuplet

\subsection{Summary}

Following the Chapters 2 and 3, the re-interpreted Rankine-Combined Vortex Model (RCVM) is now applied to the investigation of tornado over a more complex cylinder-quintuplet configuration as an extension, and the impacts of a series of tornado from different incoming directions on a building group, as well as the flow features and wind loads varies on each cylinder in presence of tornadoes are studied extensively. According to the tests, a vertical deviation of the tornado center can be captured, which results the obstacles located in accordance with the deviation trend suffer much heavier loadings. Moreover, even the distance between each cylinder in the building group is close, but the loadings exerted on each cylinder vary remarkably due to the intensive rotating effect by the disastrous tornado. An accurate measurement of the wind loads can 
be regarded as a prediction for the prevention of tornadic wind, as well as an economic but efficient approach to elucidate the underlying tornado nature. The novel conclusions derived from this study can provide further understandings of tornadic wind, and have the potential to reduce property damage before design analogous building groups.

A detailed description about the re-interpreted RCVM tornado model applied in this chapter, as well as the detailed IB-LBM implementation, has been explained in Chapter 2. Thus, only the remarks corresponding to cylinder-quintuplet model is specified in this chapter. Rest of this paper will include three more subsections. A detailed setup of tornado model from five different incoming directions over a cylinder-quintuplet is introduced in Section 4.2. Section 4.3 presents and discusses the effect of the loadings for different rotation directions of the tornado. Finally, Section 4.4 draws an extensive conclusion on the applicability of current framework.

\subsection{Setup of a cylinder-quintuplet under tornadoes from different directions}

\subsubsection{Geometry and incoming tornado directions}

As shown in Fig. 4.1(a), five cylinders numbered from 1 to 5 constitute a semi-circle-shaped configuration, and the distance between the center of the semi-circle and each cylinder is fixed as 3 times of the diameter of the cylinder D. Then tornadic wind, governed by classical RCVM is exerted on the computational domain, and expected to march toward the cylinder-quintuplet group from five representative incoming directions, which named as Cases $A$ to $E$ for convenience in following 
sections. For instance, the initial position of tornado center for case $A$ is located on an extended line connected by the center of cylinder \#3 and the semi-circle. After clockwise spun 45 degrees of the incoming tornado direction, the initial center of tornado falls to the extended line connected by the centers of cylinder \#4 and semi-circle, which turns to case B. Similarly, the incoming directions for cases $C$ to $E$ are determined using the same strategy. In order to seamlessly incorporate into present "relative motion" idea, such schematic in reality (see Fig. 4.1(a)) is able to convert into an easily implement scenario depicted in Fig. 4.1(b) during the numerical simulation. The tornado is assumed to purely rotating around its center point, meanwhile, cylinder-quintuplet configuration is allowed to translate towards the tornado center "virtually" in test cases. Numerically, the tornadic wind keeps rotational with center fixed at the center of the domain, while five cylinders translate from right to the left side in a constant velocity $v_{t}$.

\subsubsection{Dimensions of computational domain and mesh resolution}

Unless otherwise specified, mesh resolution and the computational domain applied in this part of study are fixed at $1 / 50$, and $[-40,40] \times[-15,15]$, respectively. Center of the semi-circle-shaped configuration is located at $(35,0)$, initially. The Reynolds number remains at 1000 for all cases. 


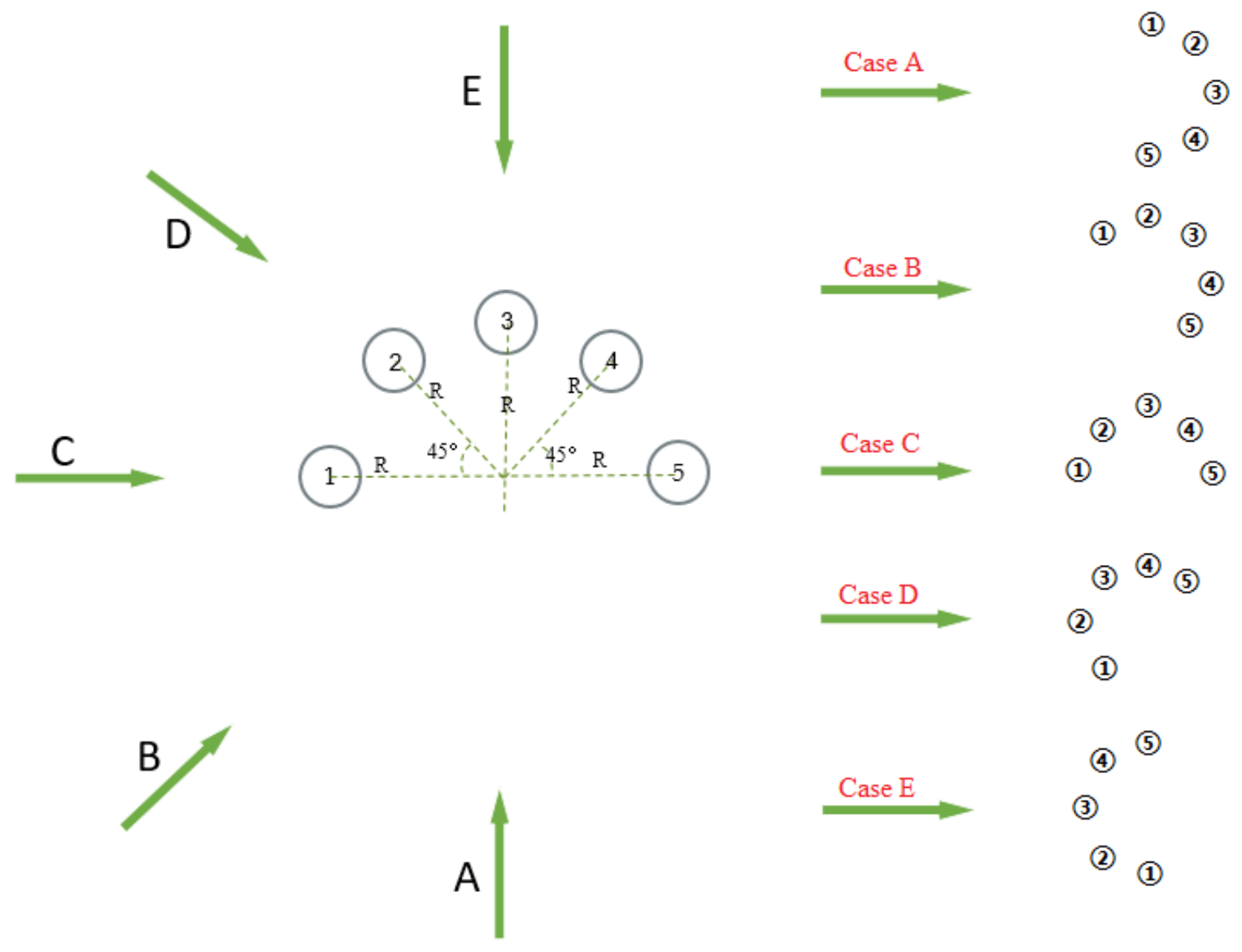

(a)

(b)

Fig. 4.1 (a) Schematic for the cylinder-quintuplet study; (b) re-tailored cylinder-quintuplet scenario in IB-LBM simulation

\subsubsection{Boundary conditions}

As illustrated in Chapter 2, immersed boundary method is applied to treat the moving boundary condition in present study, and the retailored RCVM is considered to re-interpret the boundary condition of tornadic wind. The tangential velocity of the purely rotational airflow is defined as:

$$
\vec{V}_{R}=V_{R}(\vec{j} \cos \theta-\vec{i} \sin \theta)
$$


where $\theta=\tan ^{-1}\left(\frac{y}{x}\right)$ with respect to its "virtually locked" center. A detailed computational approach about the re-tailored RCVM and its incorporation into IB-LBM framework have been described in Chapter 2.

\subsection{Numerical investigation results and discussions}

Remark that the definitions of three representative time points ("core-in", "coinciding-center", and "core-out" times) and three interaction stages (pre-interaction, primary and post-interaction stages) have been interpreted in detail in Section 2.6.1. For the sake of description convenience, these nomenclatures will continue to be adopted here.

\subsubsection{Case A}

Firstly, Case $A$ aims at simulating the tornado marching from the south of the designated building configuration as shown in Fig. 4.1(a), which transforms into the scenario depicted in Fig. 4.1(b) during the numerical experiment, supported by the re-tailored RCVM.

Fig. 4.2 and Fig. 4.3 demonstrate the streamlines and velocity magnitudes at four representative time points, including "core-in", "coinciding-center", "core-out" time and one time instant in post-interaction stage, respectively. Note that the obstacles in present simulation are regarded as "virtual translation", and the RCVM-governed tornado is assumed to be purely rotated without any translating movement. However, the realistic perspective has been employed to all figures plotted in 
this study in which the center of obstacles (cylinders) group is fixed at $(0,0)$, and the center of tornado moves with respect to the time evolution. As demonstrated in Fig. 4.2(a), before the "core-in" time point, the rotational flow streamlines ahead of the obstacles appear overall retained their original shapes governed by Eq. (4.1). However, this state is distorted in the vicinity of the building configuration due to the tornado-building interaction. Similar to single cylinder case discussed in Chapter 2, the tornado-building interaction is insignificant in the pre-interaction stage, and the affected zone is mainly observed behind the obstacles. Then the flow field, especially in the neighborhood of the cylinders, suffers drastic changes when the inner core of the tornado reaches the obstacles (primary stage) due to the intensive interaction between cylinders and tornadic wind. As visualized in Fig. 4.2(b) and (c), the streamlines around the building configuration are detected to become much asymmetric and complicated. A few wake vortexes are formed behind the cylinders, and these vortex structures change frequently, which again indicates the complex flow features occur in the primary interaction stage affected by the translational and rotational component simultaneously. After the "core-out" time point, vortex shedding phenomenon can be detected in the downward direction behind cylinder \#2 and \#4, especially for cylinder \#4 (see Fig. 4.2(d)). This observation can be regarded as another evidence that translational flow dominates the wakes of the obstacle when the cylinder-quintuplet configuration goes farther from the tornado since the rotation intensity decreases in far field of the computational domain. By comparing the velocity magnitudes plotted in Fig. 4.3 (a) - (d), the highest velocity is right in front of cylinder \#1 in the pre-interaction 
stage, and then, in the primary and post-interaction stages, the zone with highest velocity is shifted into the wake region. Moreover, this highest velocity zone is observed to exhibit an upward trend during the interaction process.

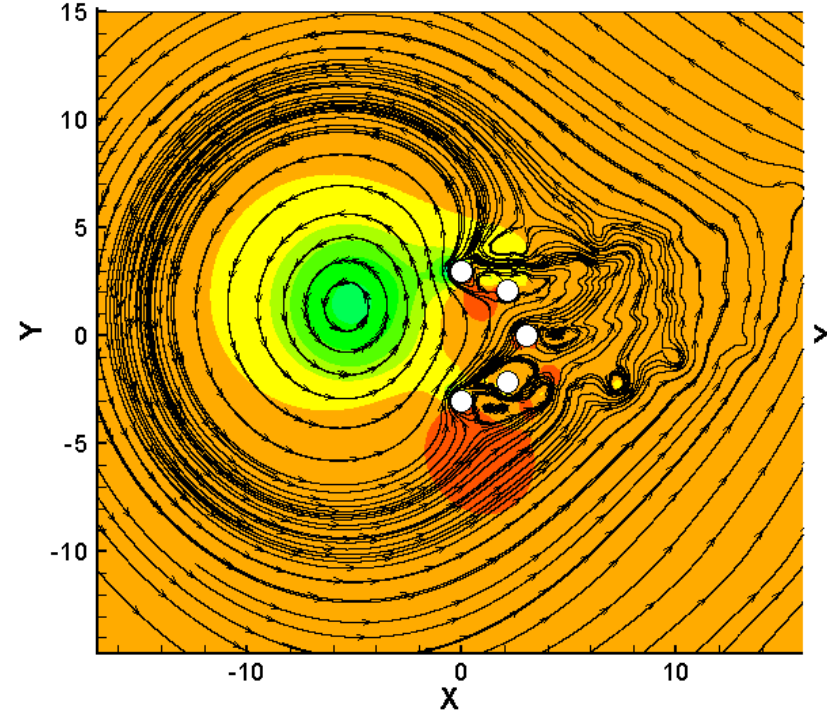

(a)

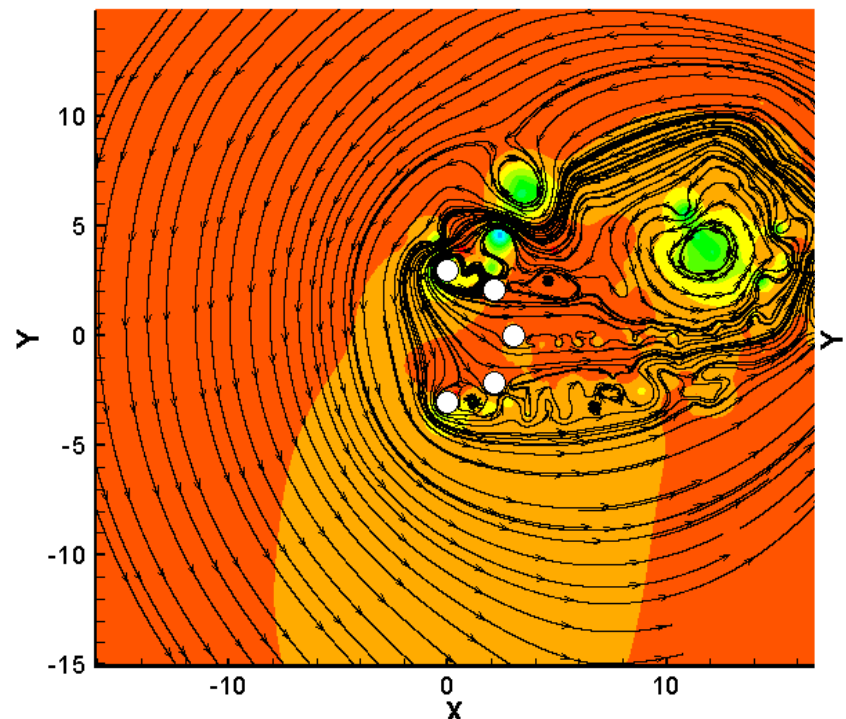

(c)

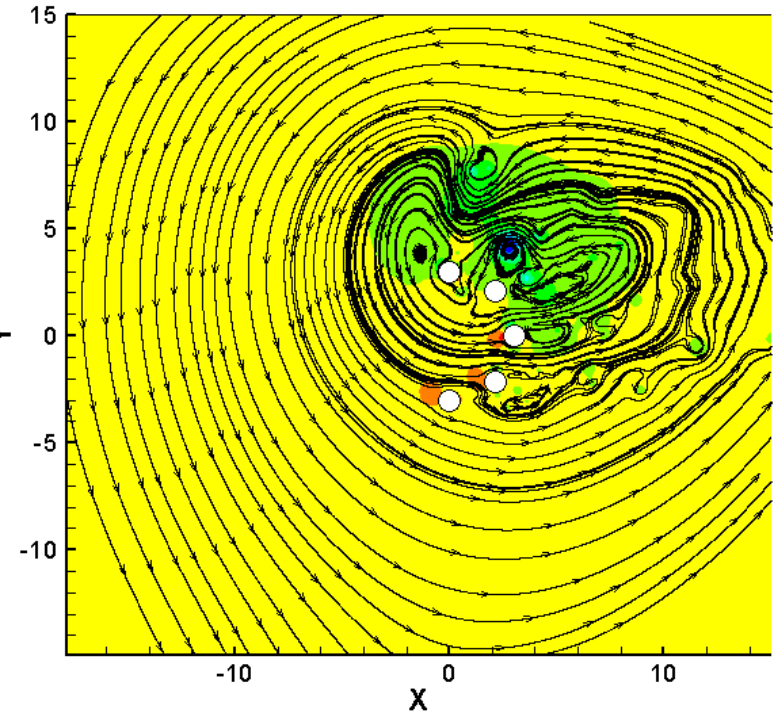

(b)

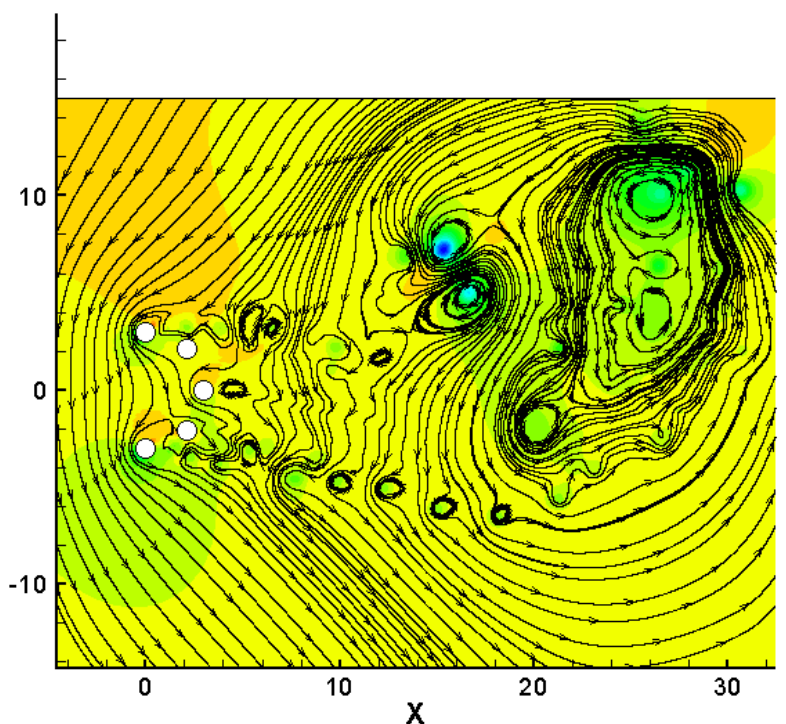

$(\mathrm{d})$

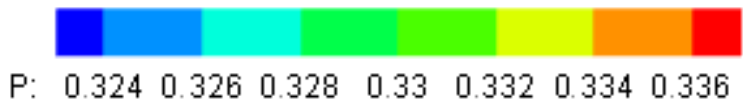

Fig. 4.2 Streamline contour for Case A at four representative time instants: (a).at right $r_{c}$; (b). tornado center; (c). at left $r_{c}$; (d). departed tornado) 


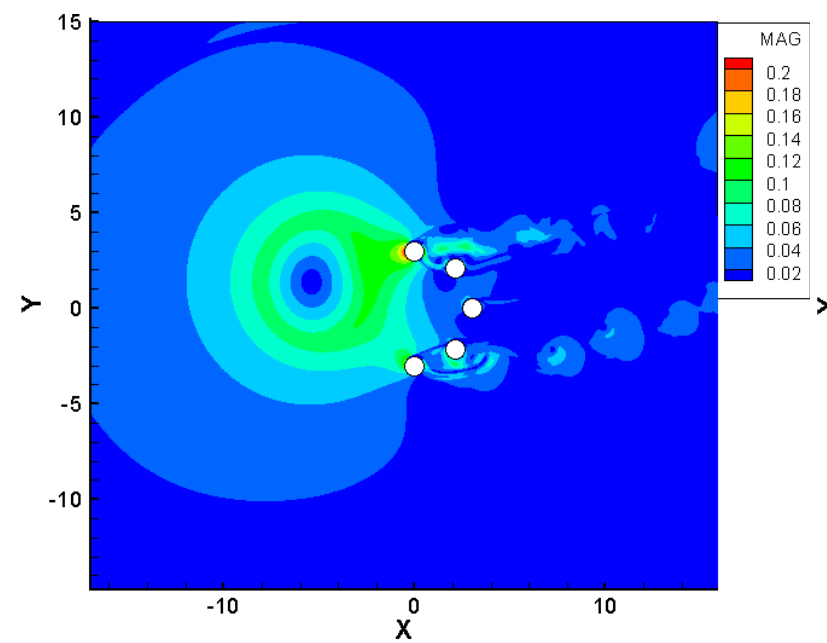

(a)

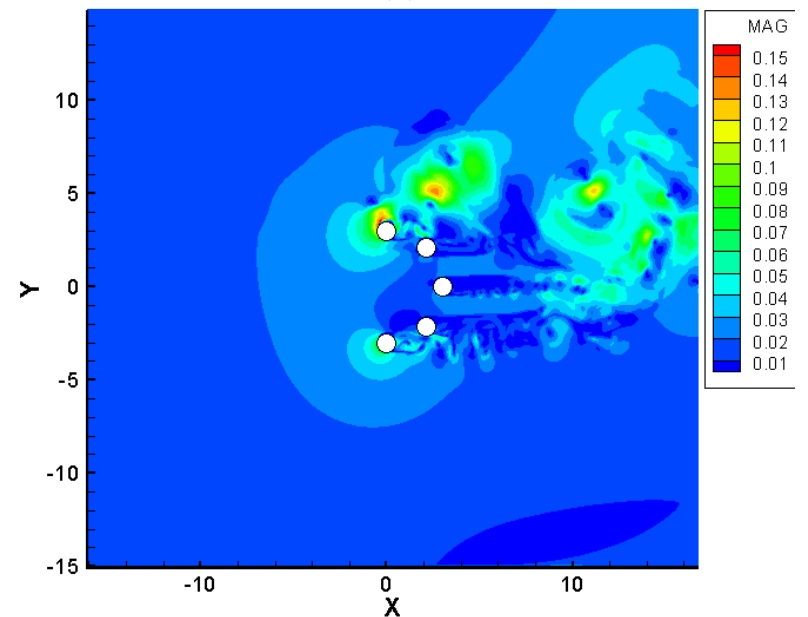

(c)

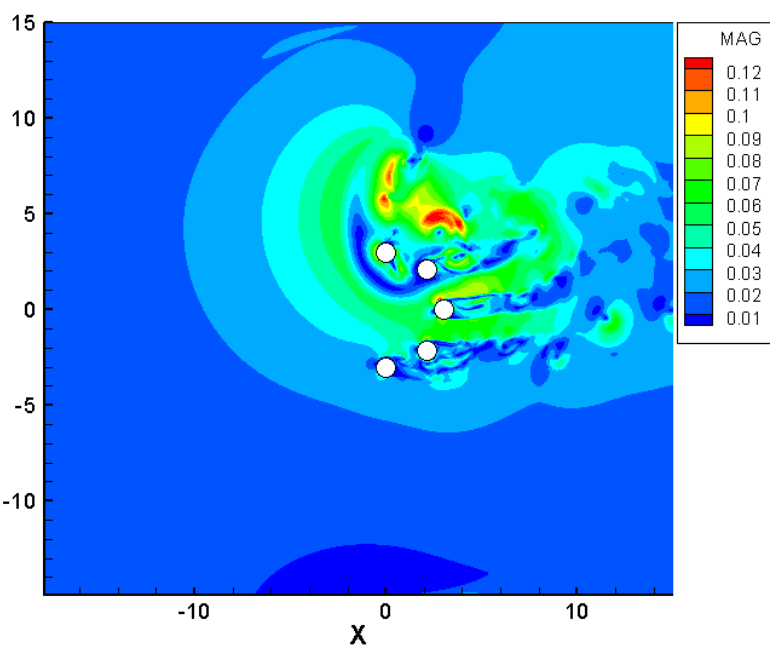

(b)

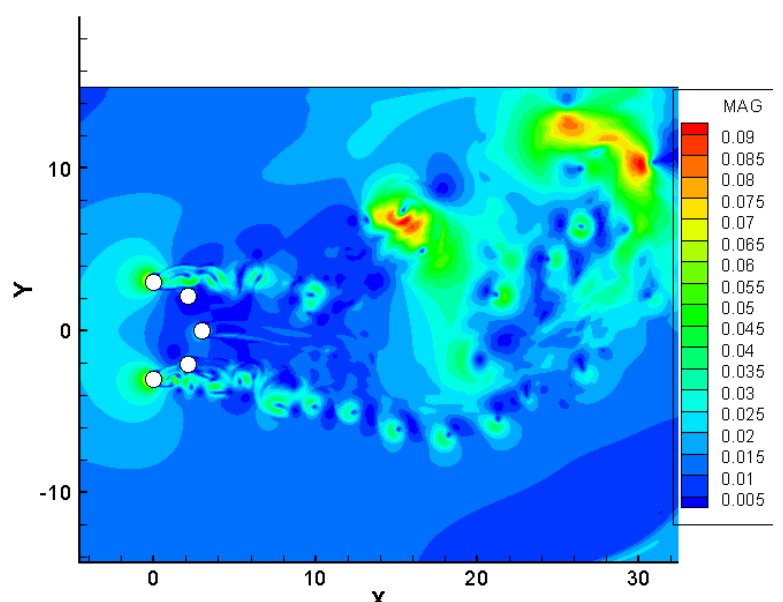

(d)

Fig. 4.3 Velocity magnitude for Case A at four representative time instants: (a).at right $\mathrm{r}_{\mathrm{c}}$; (b). tornado center; (c). at left $r_{c}$; (d). departed tornado)

Besides, the loadings on each cylinder can also be analyzed through evolution of $C_{x}, C_{y}$, and $C_{m}$.

As depicted in Fig. 4.4 - Fig. 4.6, in the pre-interaction stage $(x<-3)$, all three coefficients of cylinder \#1 and \#5 start to decrease and vary in a relative stable trend at the beginning $(x<-15) . C_{x}$, $C_{y}$, and $C_{m}$ exerted on cylinder \#1 and \#5 are observed to be almost the same. Then when the tornadic wind gets close to the cylinders (about $x=-13$ ), the force coefficient curves for cylinder \#1 and \#5 start to separate from each other, and the slope of each curve for cylinder \#1 is 
significantly larger than that for cylinder \#5, while coefficients for other three cylinders vary within a relative small range. These can be explained by the following two reasons: firstly, front obstacles, which located closest to the incoming tornadic wind are supposed to endure higher loadings than those located aside the front buildings, and this phenomenon is named as "front effect" for convenience; secondly, the tornado center exhibits a slight upward in the pre-interaction and primary stage, which is attributed to the counterclockwise rotational flow component in this tornadic wind. Consequently, the extremum value and variation trend of $C_{x}, C_{y}$, and $C_{m}$ for cylinder $\# 1$ are much larger than those for cylinder \#5. This observation is named as "deviation effect". Along with "front effect", these two effects can be adopted not only in this specific case, but also fit all five test cases in current cylinder-quintuplet simulations, which will be examined and discussed in following sub-sections.

As time elapses, $C_{x}, C_{y}$, and $C_{m}$ reach their first extremum point in the vicinity of "core-in" point $(x=-3)$. Taking the extremum $C_{x}$ as an observation point, it can be noticed that $C_{x}$ for cylinder $\# 1$ is almost 3 times higher than that for cylinder \#5, and forces exerted on other three cylinders are quite small. This feature indicates cylinder \#1 endures the largest loadings in Case A configuration. After the loadings reach the extremum point at "core-in" time, $C_{x}, C_{y}$, and $C_{m}$ for each cylinder start to increase, and the slope for each curve remains at a relative high value due to the intensive interaction during the primary stage. Then, a second turning point is observed near $x=9$ in $C_{x}, C_{y}$, and $C_{m}$ curves, which indicates that heavy loadings still exist even the tornado moves away from the 
building.

Even hidden behind cylinder \#1 and \#5, cylinder \#2 and \#4 still call for attention. In the post-interaction stage $(x>10)$ when the tornado goes more distant, $C_{x}, C_{y}$, and $C_{m}$ for these two cylinders show periodically oscillating (see Fig. 4.4 - Fig. 4.6), which look like curves when purely translational flow passes single cylinder, and the amplitude of cylinder \#4 is larger than that of cylinder \#2. This observation can be further verified by shedding vortex in the wake region in Fig. 4.2(d), indicating these two cylinders are dominated by the translational velocity and less affected by the rotating tornadic wind, in particular in the lower half of the configuration.

In general, cylinder \#1 endures the largest loading during the tornadic wind simulation, followed by cylinder \#5. The building first reached by the tornado is considered to be more affected by the rotational component than the hidden buildings, especially when the tornado moves upward toward the building due to "deviation effect". On the other hand, cylinders located opposite to the incoming tornado, such as \#2 - \#4 in this case, are mainly dominated by translating component, and these cylinders are identified to be less affected by the strong rotating tornado. These conclusions will be further examined in the following cases with different incoming directions.

Next, in order to assess how the mesh resolution and domain size affect the cylinder-quintuplet case, numerical tests corresponding to Case A are performed using meshes with different resolutions and $X$-dimension lengths. Besides the computational domain $[-40,40] \times[-15,15]$ with $1 / 50$ mesh resolution discussed above, another two sets of experiments, including computational domain [-30, 


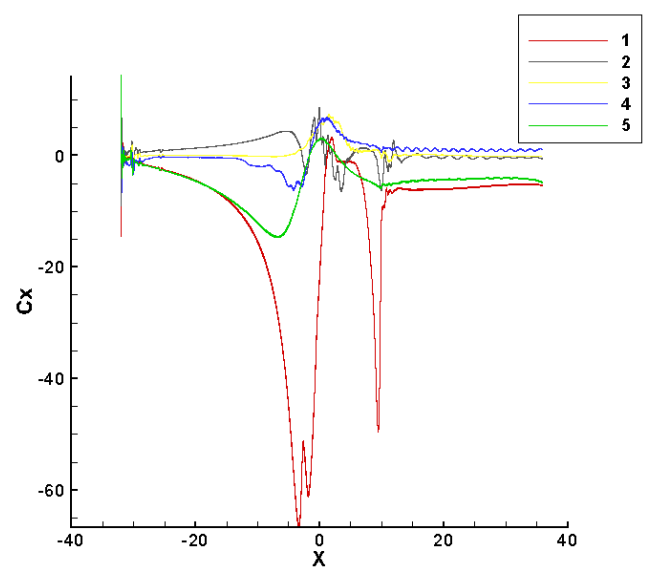

Fig. 4.4 X-direction force coefficient for Case A

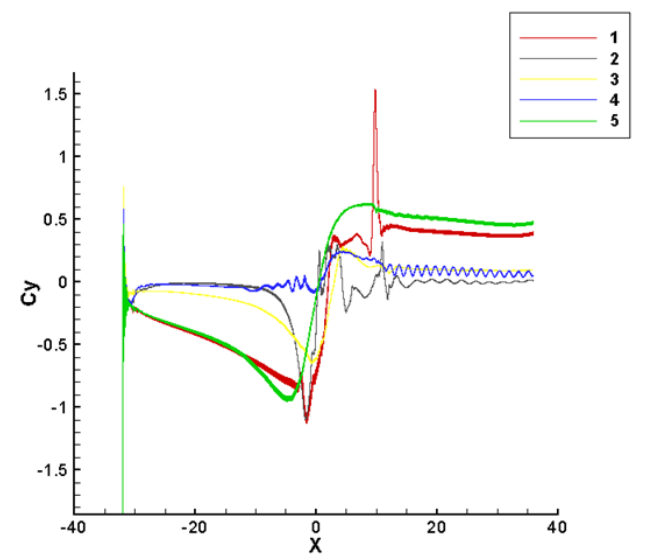

Fig. 4.5 Y-direction force coefficient for Case A

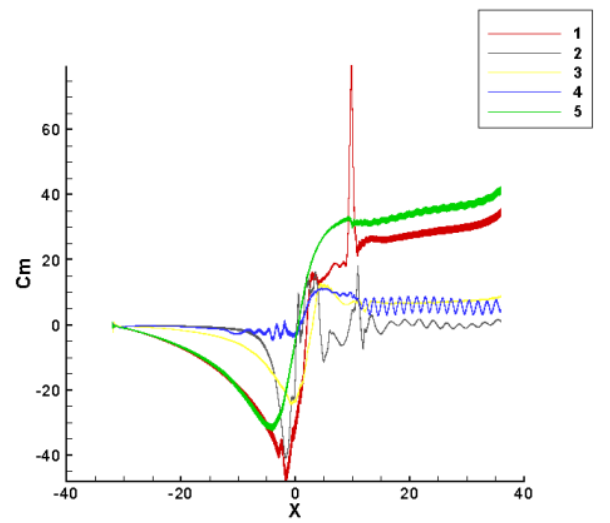

Fig. 4.6 Moment coefficient for Case A 
$30] \times[-15,15]$ with $1 / 50$ mesh resolution, and $[-30,30] \times[-15,15]$ with $1 / 60$ mesh resolution, are studied as well, and other parameters are fixed. By comparing the evolution of three dimensionless coefficients $C_{x}, C_{y}$, and $C_{m}$ shown in Fig. 4.7, the overall trend for these three sets of test cases are the same, and only the extreme value for each case varies in small ranges due to the $X$-dimension and mesh resolution impacts. According to the tests, regardless of mesh resolution and computational dimension, all the features regarding the interaction between tornado and the obstacles are proved to be reliable.
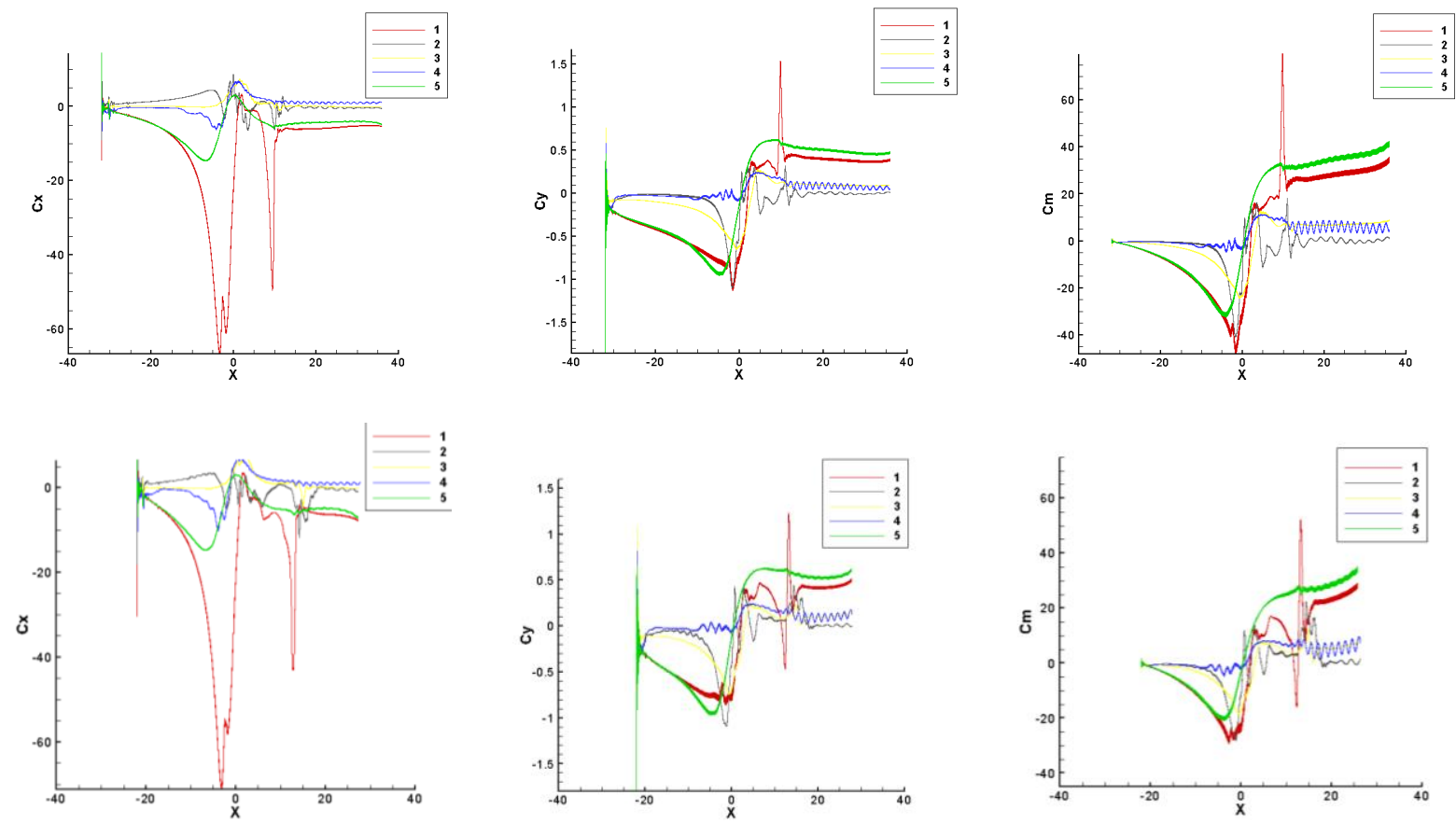

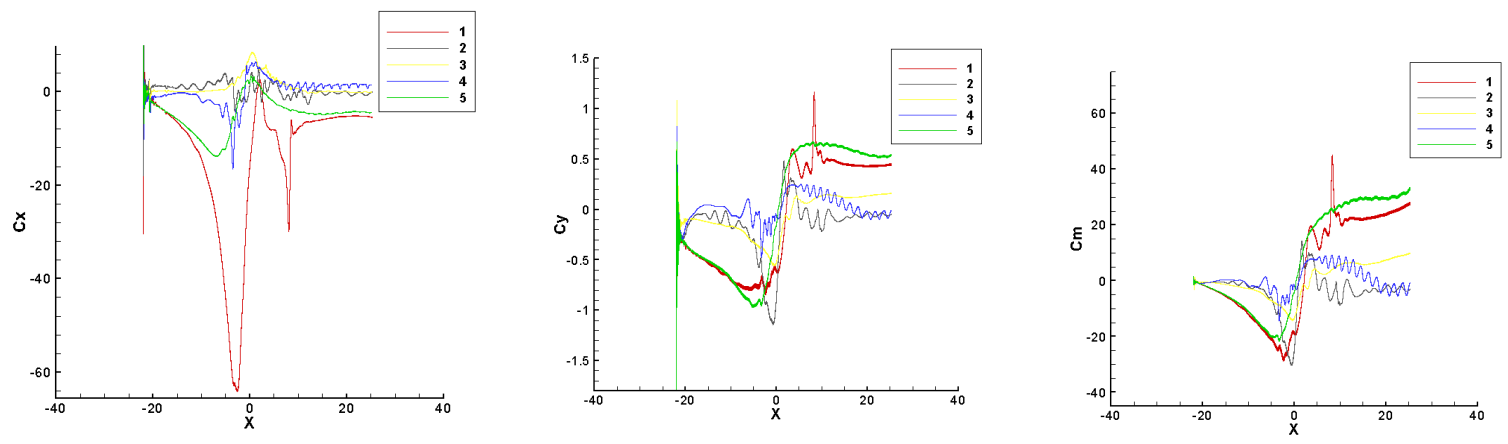

Fig. 4.7 Three sets of testing cases for Case A with different mesh resolution and $\mathrm{X}$-dimensional size

(Notes: first row represents the $\mathrm{C}_{\mathrm{x}}, \mathrm{C}_{\mathrm{y}}, \mathrm{C}_{\mathrm{m}}$ for domain $[-40,40] \times[-15,15]$ with mesh resolution 1/50; second row represents the $C_{x}, C_{y}, C_{m}$ for domain $[-30,30] \times[-15,15]$ with mesh resolution $1 / 50$; third row represents the $C_{x}, C_{y}, C_{m}$ for domain $[-30,30] \times[-15,15]$ with mesh resolution 1/60;

\subsubsection{Case B}

Next, the cylinder-quintuplet turns 45 degrees in counter-clockwise direction on the basis of Case A, which is equivalent to clockwise spinning 45 degrees for the incoming direction of tornado in reality. The streamlines and velocity magnitudes for four representative time instants are depicted at Fig. 4.8 and Fig. 4.9. Similar to Case A, until "core-in" time point, the streamlines in the vicinity of the obstacles are discovered to be distorted due to the tornado-obstacles interaction, while streamlines far away from the obstacles still maintain the undisturbed profile. An upward trend of the tornadic wind is again observed in pre-interaction stage (see Fig. 4.8 (a)), which results in the force loadings exerted on cylinder \#1 and \#2 significantly higher than those on other cylinders (see Fig. 4.10- Fig. 4.12). Despite the "deviation effect" attributed to the high loadings on cylinder \#1 and \#2, the location of these two cylinders should be considered seriously as well. Recall that 
cylinder \#1 and \#5 are the two obstacles enduring the highest loadings in Case A due to the "front effect". Analogically, cylinder $\# 1$ and $\# 2$ are the two obstacles closest to tornado among the five cylinders for this case. Therefore, based on these two effects, they are believed to endure highest loadings during the tornado-buildings interaction undoubtedly, in particular cylinder \#1.

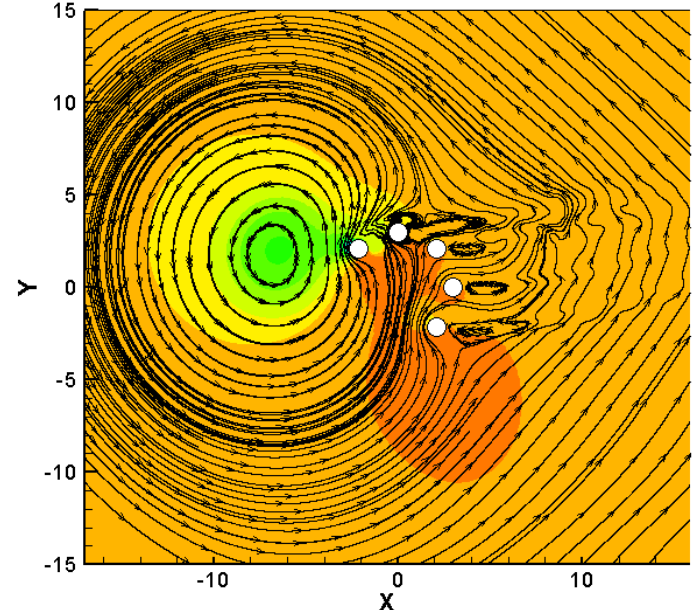

(a)

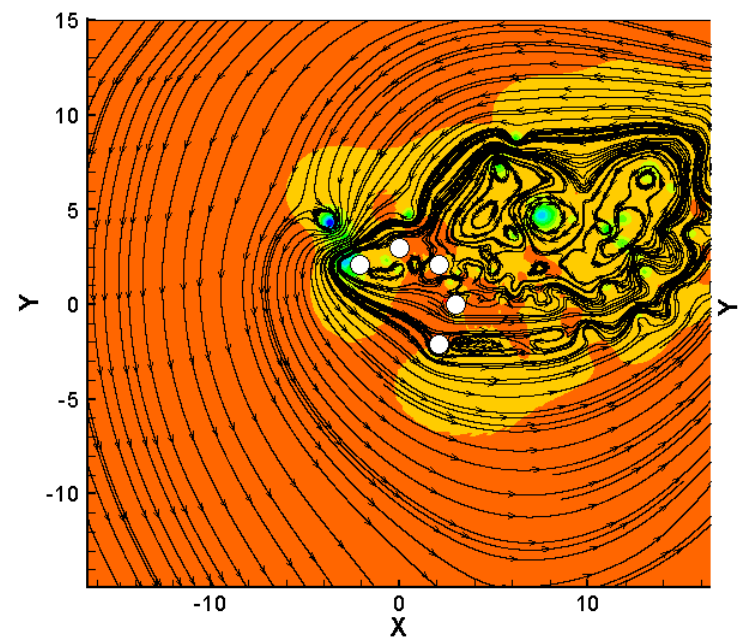

(c)

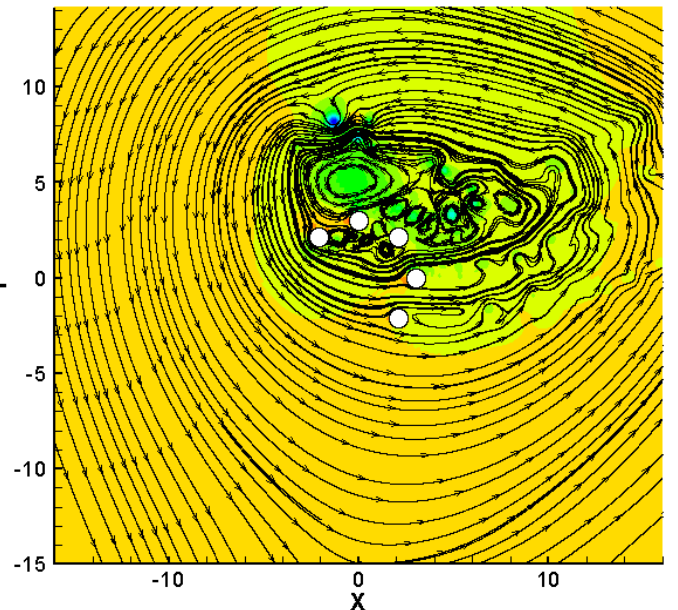

(b)

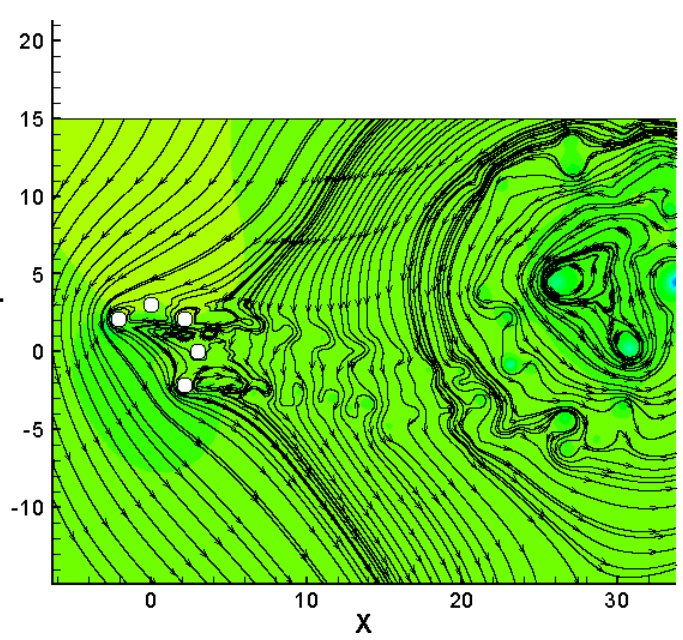

(d)

P: $\begin{array}{llllllll}0.324 & 0.326 & 0.328 & 0.33 & 0.332 & 0.334 & 0.336\end{array}$

Fig. 4.8 Streamline contour for Case B at four representative time instants: (a).at right $r_{c}$; (b). tornado center; (c). at left $\mathrm{r}_{\mathrm{c}}$; (d). departed tornado) 


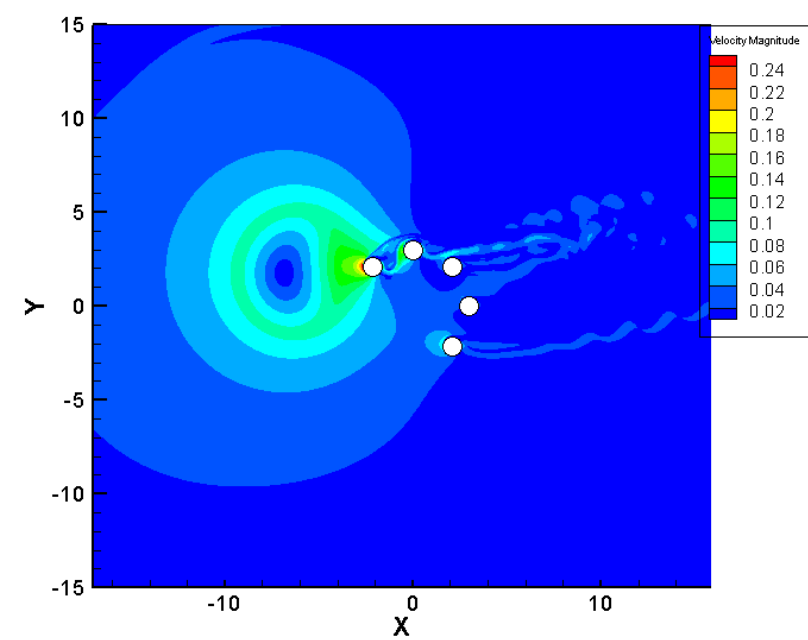

(a)

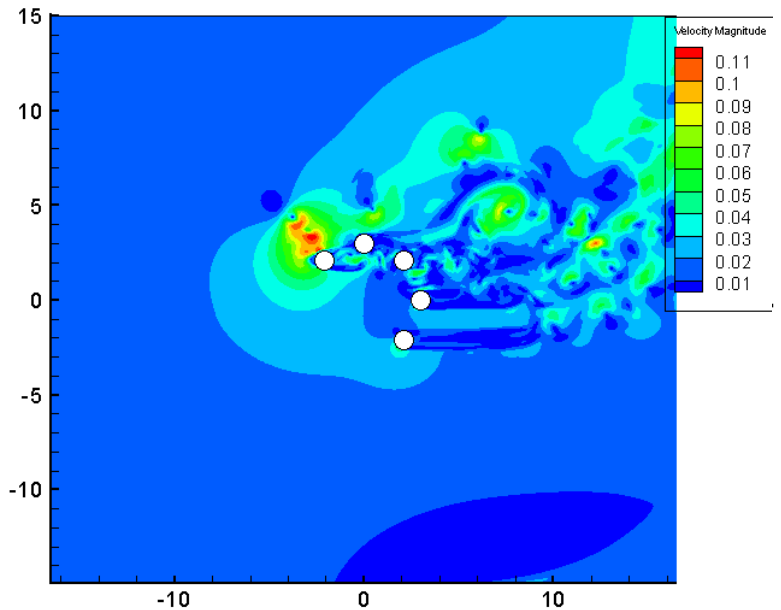

(c)

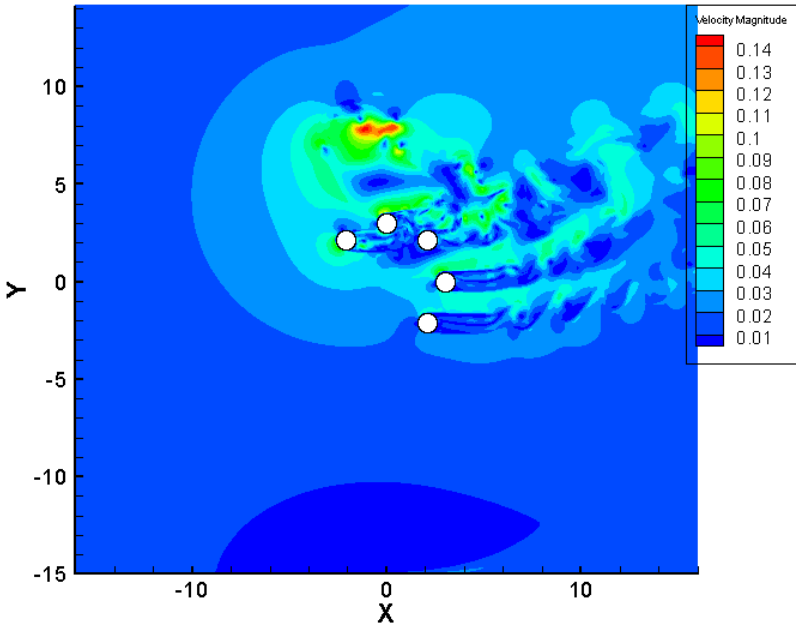

(b)

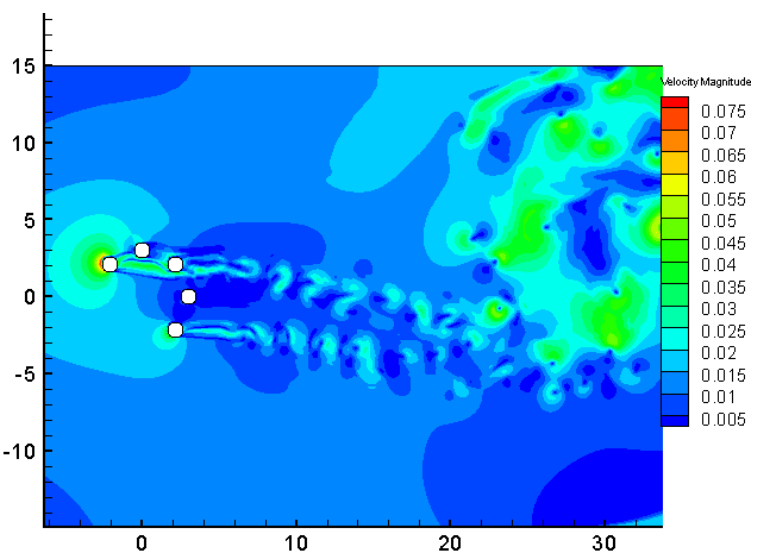

(d)

Fig. 4.9 Velocity magnitude for Case B at four representative time instants: (a).at right $\mathrm{r}_{\mathrm{c}}$; (b). tornado center; (c). at left $\mathrm{r}_{\mathrm{c}}$; (d). departed tornado)

As shown in Fig. 4.10- Fig. 4.12, $C_{x}, C_{y}$, and $C_{m}$ for each cylinder reach the extremum value near "core-in" point, and then vary drastically until the second turning point at around $\mathrm{x}=0$. Again, another extremum point is re-emerged on cylinder \#1 near $x=8$ (see Fig. 4.10- Fig. 4.12) in the post-interaction stage, confirming that the tremendous loadings last until the obstacle completely 
detached the inner core of the tornadic wind. After the tornado goes distant, rotational component no longer dominates the domain, thus, the force coefficients restore to relative stable states after $x>10$.

After analyzing the upper half of the configuration (cylinder \#1 and \#2), other three cylinders (cylinder \#3, \#4, and \#5) located at the lower half will be discussed. Neither "front effect" nor "deviation effect" can be applied to analyze the loadings on these three cylinders. Consequently, the influence caused by the rotation can be reduced greatly, and the trends of $C_{x}, C_{y}$, and $C_{m}$ on cylinder \#3, \#4, and \#5 remain much more stable than those on cylinder \#1 and \#2 during the whole procedure. These discoveries imply that the impacts of tornado on different obstacles in one configuration can vary significantly and appropriate arrangement of buildings is of vital importance to prevent tornadic wind. Moreover, when the tornado goes distant, frequent oscillation can be observed at the end stage of the evolution figures for cylinders \#3, \#4, \#5 (see Fig. 4.8(d), Fig. 4.9(d)), which further indicates that the translational velocity dominates the flow at post-interaction stage.

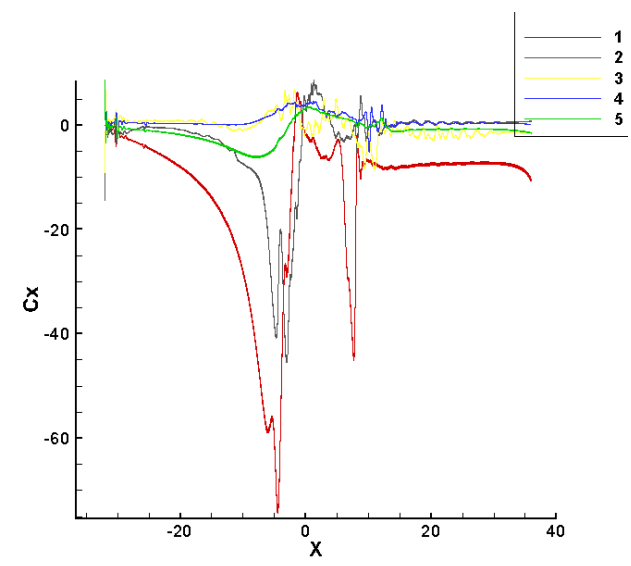

Fig. 4.10 X-direction force coefficient for Case B 


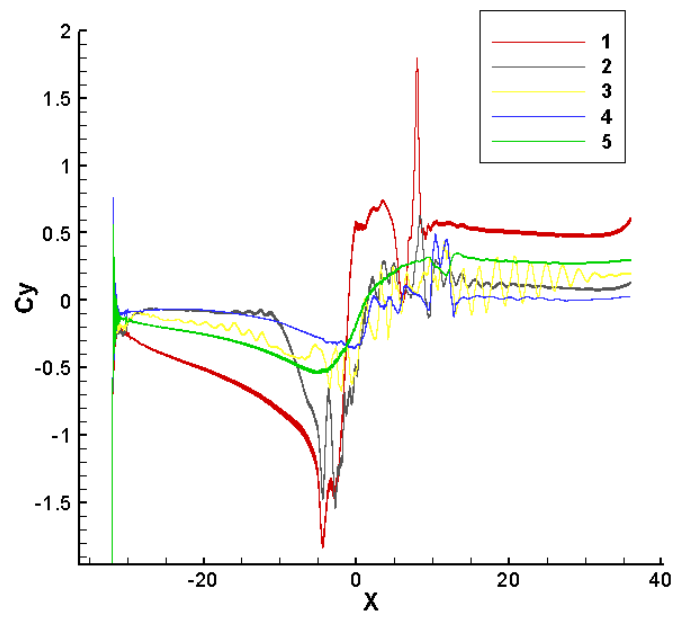

Fig. 4.11 Y-direction force coefficient for Case B

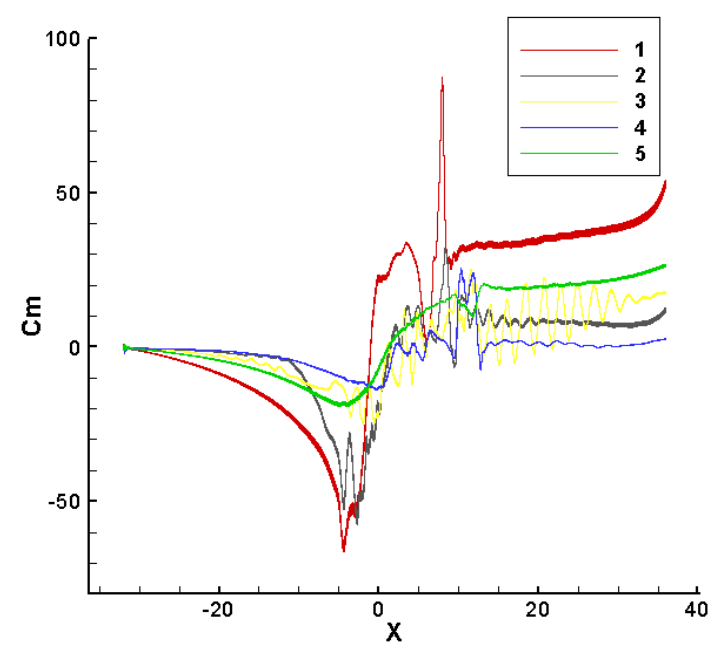

Fig. 4.12 Moment coefficient for Case B

\subsubsection{Case C}

With spinned 45 more degrees in anti-clockwise direction on the basis of Case B, the center of tornadic wind, cylinder \#1 and \#5 are in the same horizontal level, and cylinder \#1, \#2, and \#3 are in front half of the configuration, while other two cylinders located behind them. Thus, according to the "front effect" concluded in Section 4.3.1, cylinder \#1, \#2, and \#3 are expected to reach the inner 
core of tornadic wind prior to cylinder \#4 and \#5, which leading to higher loadings on the front three obstacles, and the velocity magnitude of cylinder \#1, \#2, and \#3 is obviously larger than that of cylinder \#4 and \#5 in pre-interaction and primary stage (as shown in Fig. 4.14(a) and Fig. 4.14(b)). Then taking "deviation effect" into consideration, with the cylinder-quintuplet marching towards the tornado, upward trend of the tornadic wind is still discovered, which results several irregular vortex found behind and upon the obstacles (see Fig. 4.13(b) and (c)). These features indicate drastic interaction between the tornado and cylinder \#2 and \#3. Fig. 4.15 demonstrates the force coefficient evolution in $x$-direction, and note that three curves representing cylinder $\# 1$, \#2, and \#3 tend to alleviate rapidly in pre-interaction stage along with tornado close to the obstacles $(x=-3)$, and then they reach the extremum point in sequence from \#1 to \#3. Even the three cylinders reach the "core-in" point at different time depending on their relative locations, the extremum values of $C_{x}$ for these three cylinders are quite similar. However, the force coefficient in $y$-direction and moment coefficient on cylinder \#2 are observed to be larger than cylinder \#1 and \#3, which is attributed to upward trend of tornadic wind. Thus, cylinder \#2 endures higher loadings than cylinder \#1 located at the horizontal level and \#3 in top of the configuration. It can be concluded that $C_{x}$ is largely determined by the combined effects of "deviation effect" and "front effect"; however, the value of $C_{y}$ and $C_{m}$ are mainly determined by "deviation effect". 


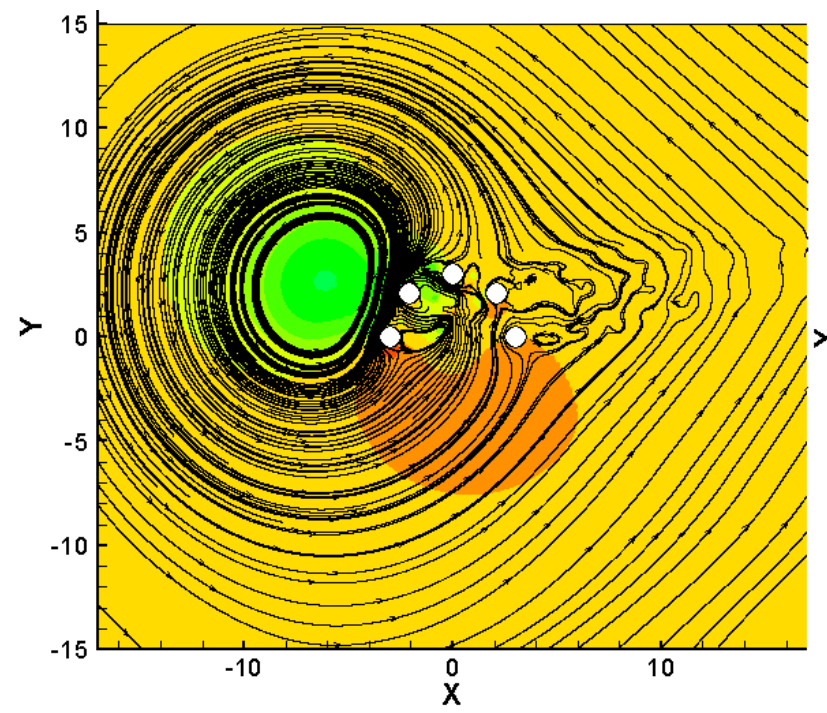

(a)

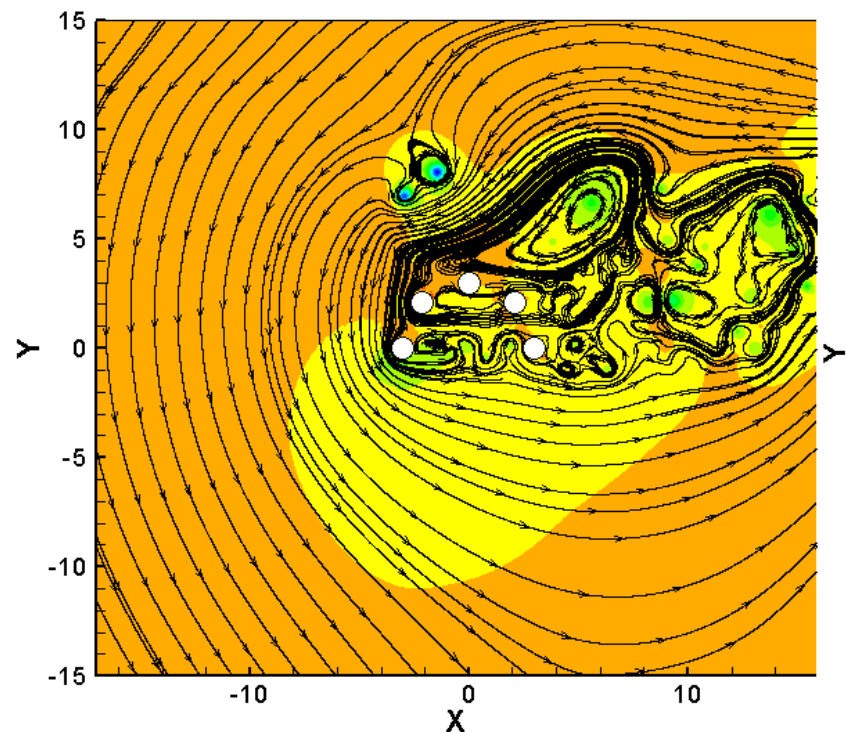

(c)

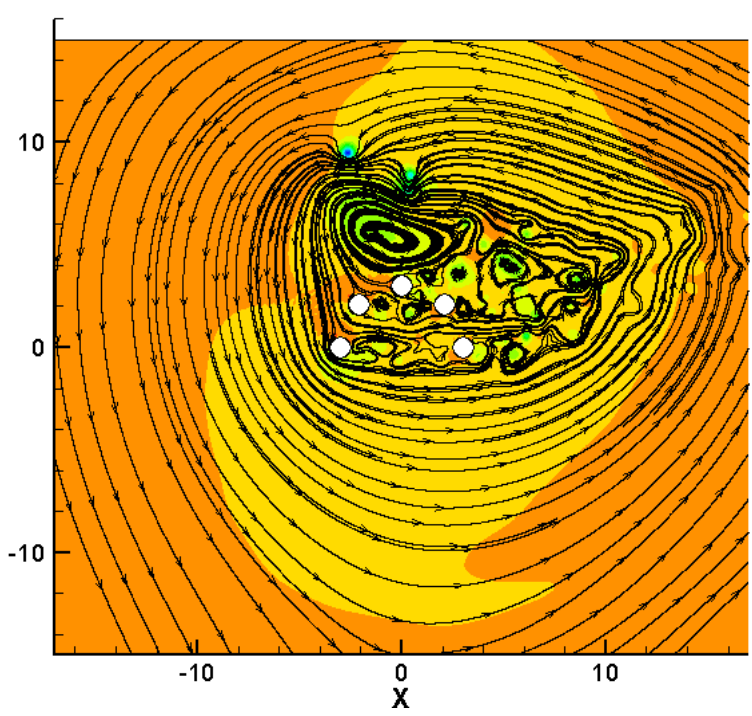

(b)

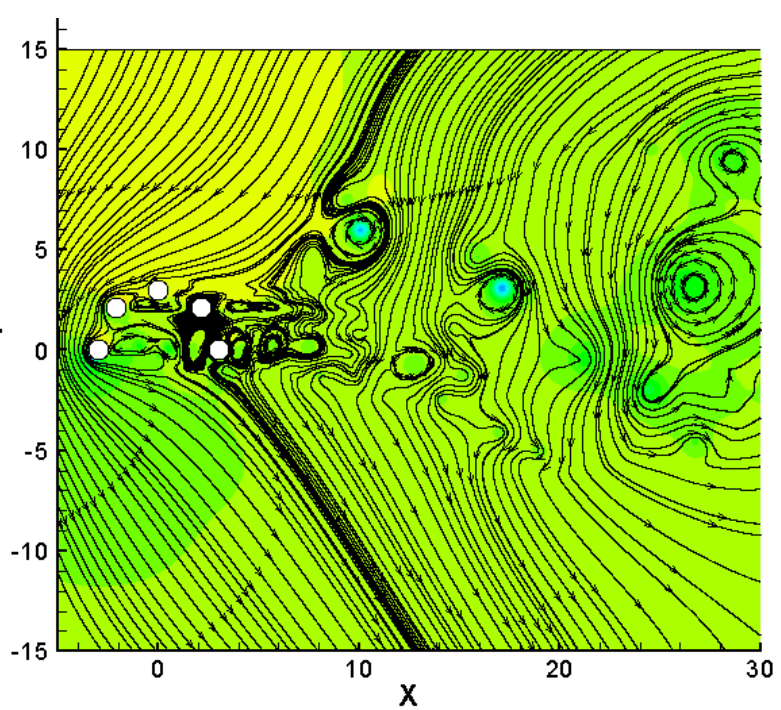

(d)

P: $\begin{array}{lllllll}0.324 & 0.326 & 0.328 & 0.33 & 0.332 & 0.334 & 0.336\end{array}$

Fig. 4.13 Streamline contour for Case $C$ at four representative time instants: (a).at right $r_{c}$; (b). tornado center; (c). at left $r_{c}$; (d). departed tornado) 


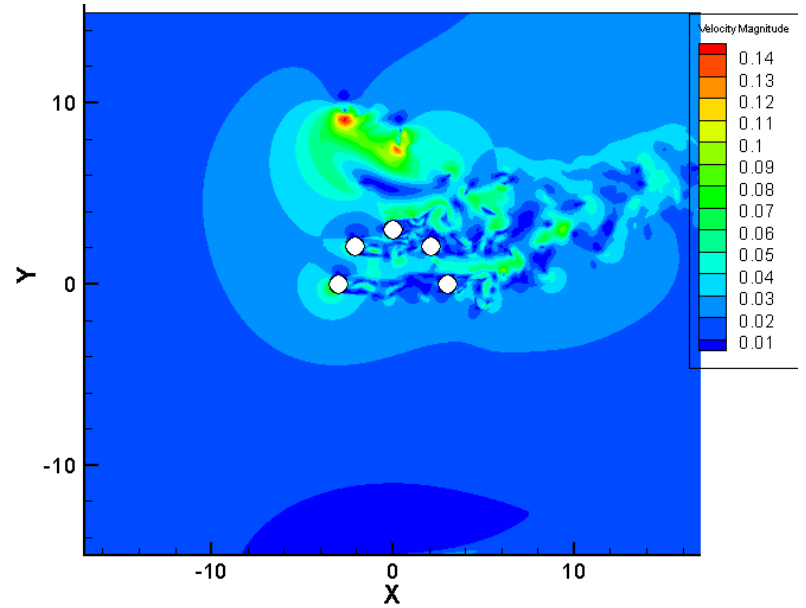

(a)

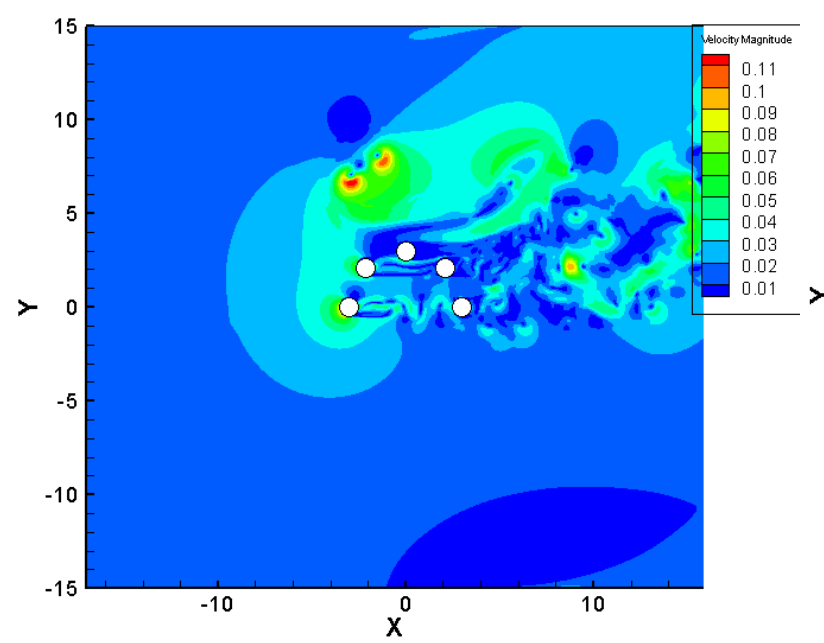

(c)

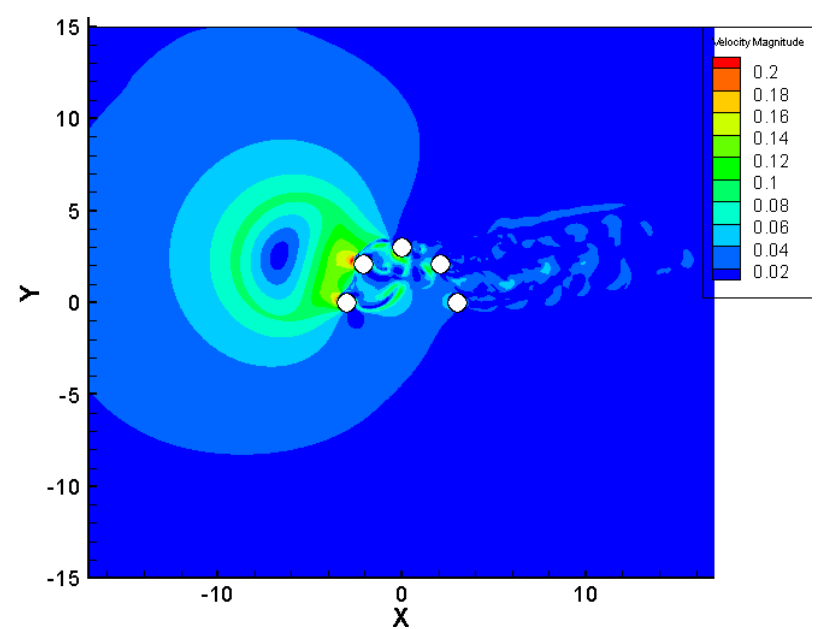

(b)

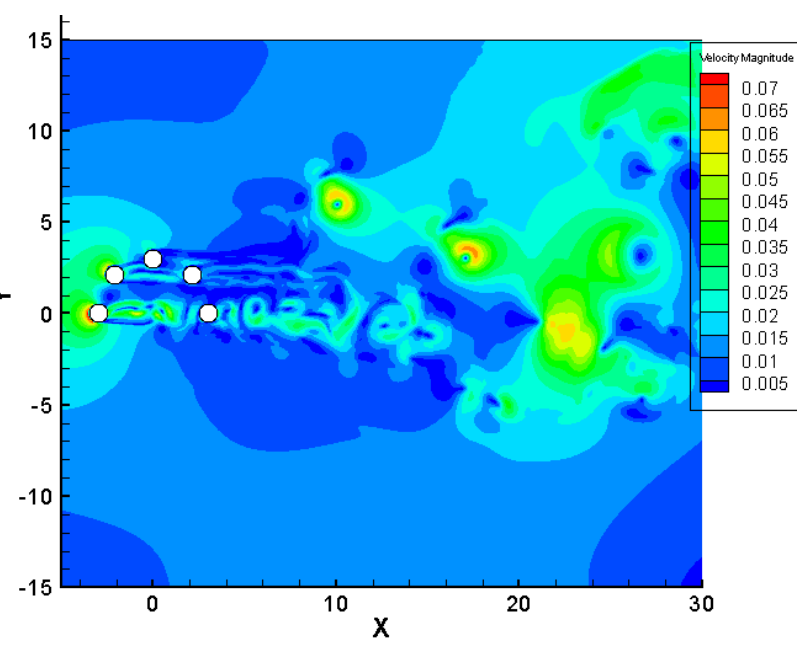

(d)

Fig. 4.14 Velocity magnitude for Case $\mathrm{C}$ at four representative time instants: (a).at right $\mathrm{r}_{\mathrm{c}}$; (b). tornado center; (c). at left $r_{c}$; (d). departed tornado)

Compared to the heavy loadings on front three cylinders, loadings on cylinder \#4 and \#5 are approximately only one third of cylinder \#1 - \#3 in $x$-direction. Translational flow dominates the wake of cylinder \#4 and \#5 as they located beside the front three cylinders. Compared with cylinder \#4, cylinder \#5 shows clearly frequent oscillation in the wake of the flow. Closed to rotational dominance cylinder \#3, the wake pattern for cylinder \#4 is distorted by cylinder \#3 and the 
moved-up tornado center (see Fig. 4.14(d)). However, cylinder \#5 undergoes weak influence since it is away from the cylinder \#1-\#3 group. Thus the oscillation phenomenon can be detected clearly behind the cylinder \#5.

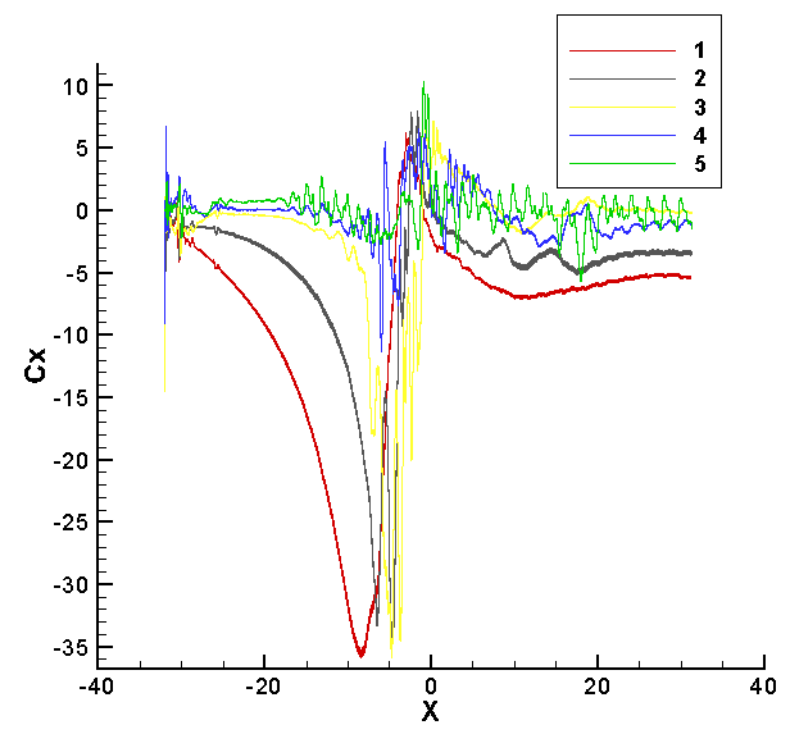

Fig. 4.15 X-direction force coefficient for Case C

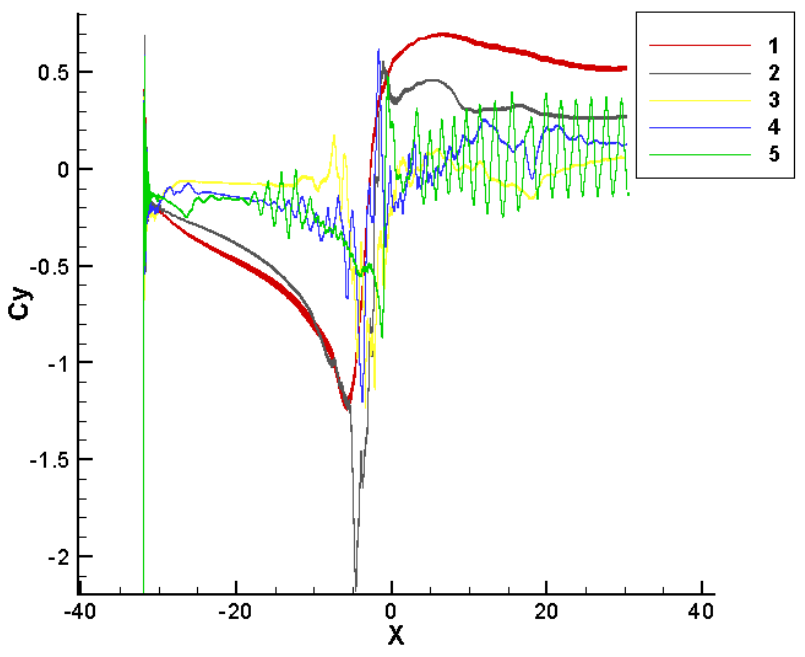

Fig. 4.16 Y-direction force coefficient for Case C 


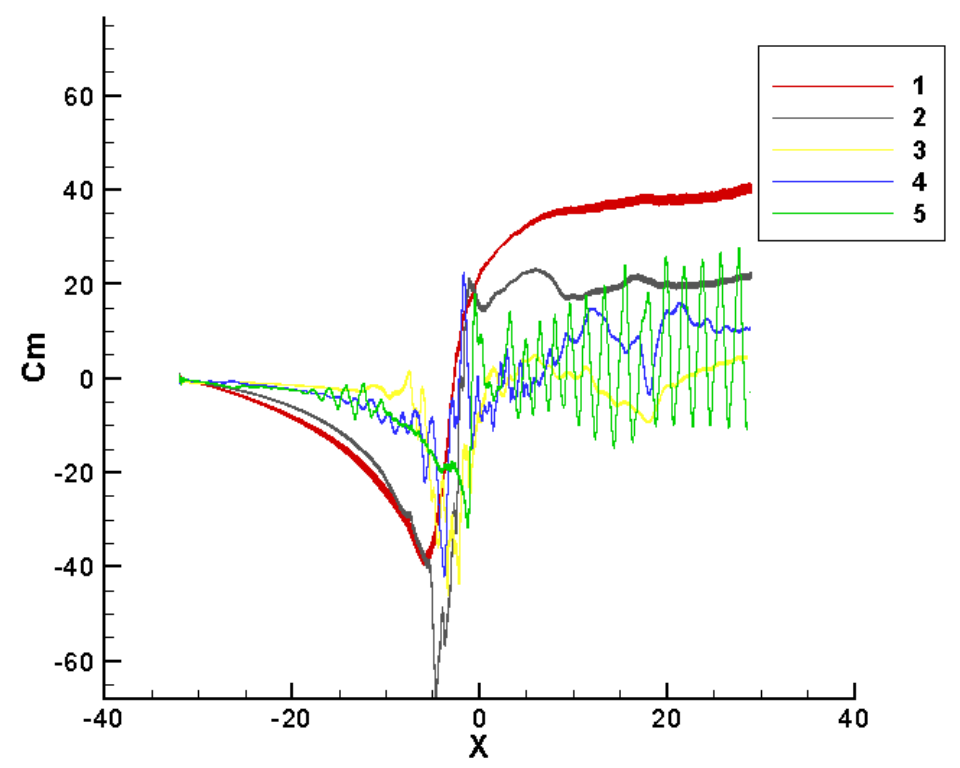

Fig. 4.17 Moment coefficient for Case C

\subsubsection{Case D}

As the cylinder-quintuplet continue to spin 45 degrees on anti-clockwise direction, cylinder \#2 becomes the front obstacle in this situation, and cylinder \#3 and \#4 move to the upper half as well. Again, according to the aforementioned "front effect", cylinder \#2 is affected by the rotational tornado prior to other cylinders ahead of other cylinders due to its front location. Then taking "deviation effect" into consideration, the tornado center is observed to exhibit an upper trend, and gets close to cylinder \#3 and \#4 located at upper side (see Fig. 4.18(a)) in sequence. These observations manifest that cylinder \#2, \#3, and \#4 obviously endure higher loadings than cylinder $\# 1$ and \#5, which is proved again in Fig. 4.20 - Fig. 4.22. Similar to Case C, the evolution of $x$-direction force coefficient for cylinder \#2, \#3, and \#4 tends to be very close to each other, and they 
reach the first turning point near "core-in" time instant in sequence from \#2 to \#4 with respect to the position for each cylinder. Likewise, the force coefficient in $y$-direction and moment coefficient on cylinder \#3 are detected to be larger than cylinder \#2 and \#4, which are obviously attributed to the superposition of "deviation effect" and "front effect", as discussed in Case C. Another two cylinders \#1 and \#5 are located at the bottom and right side of the configuration, respectively. The extremum values of $C_{x}, C_{y}$, and $C_{m}$ on these two cylinders are not as high as the front three. Force loadings on cylinder \#1 and \#5 in $x$-direction are discovered to be approximately only $1 / 3$ than those exerting on cylinder \#2, \#3 and \#4. Also, the shedding of vortices can be monitored in the downward direction in the post-interaction stage (see Fig. 4.18(d) and Fig. 4.19(d)) and the translating flow is believed to dominate the domain at the end stage when the cylinder-quintuplet moves away from the tornado. 


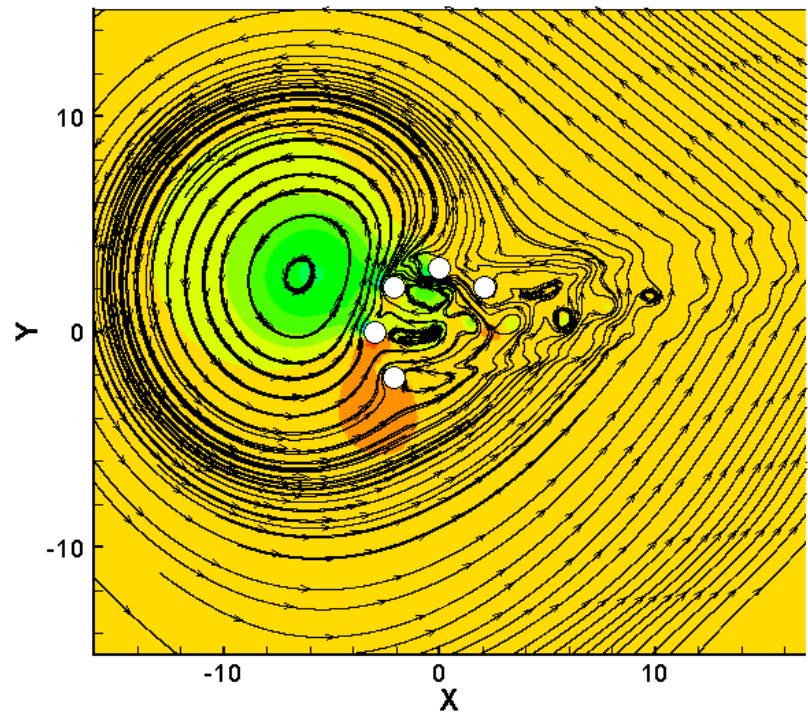

(a)

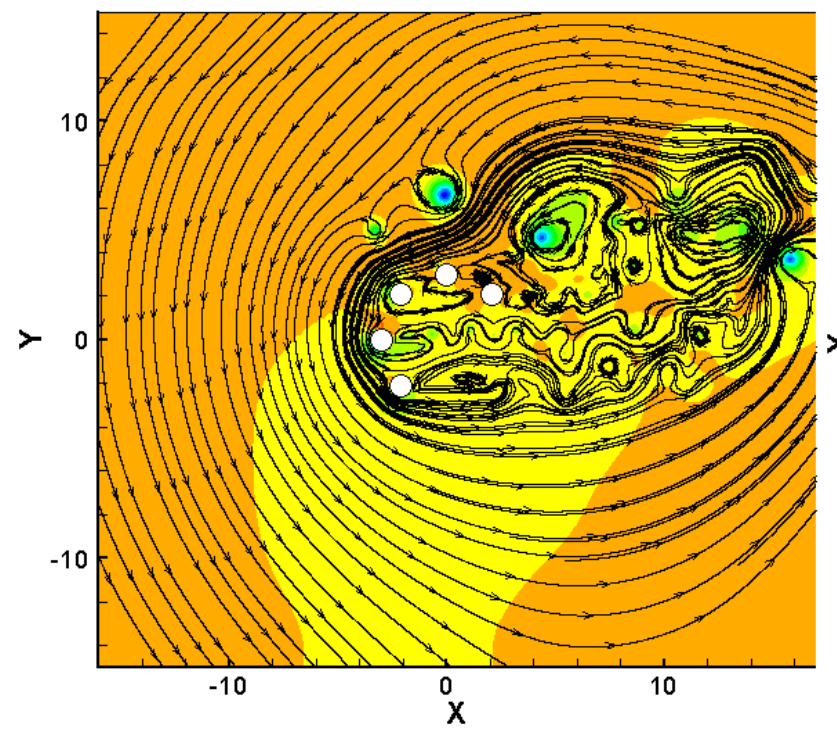

(c)

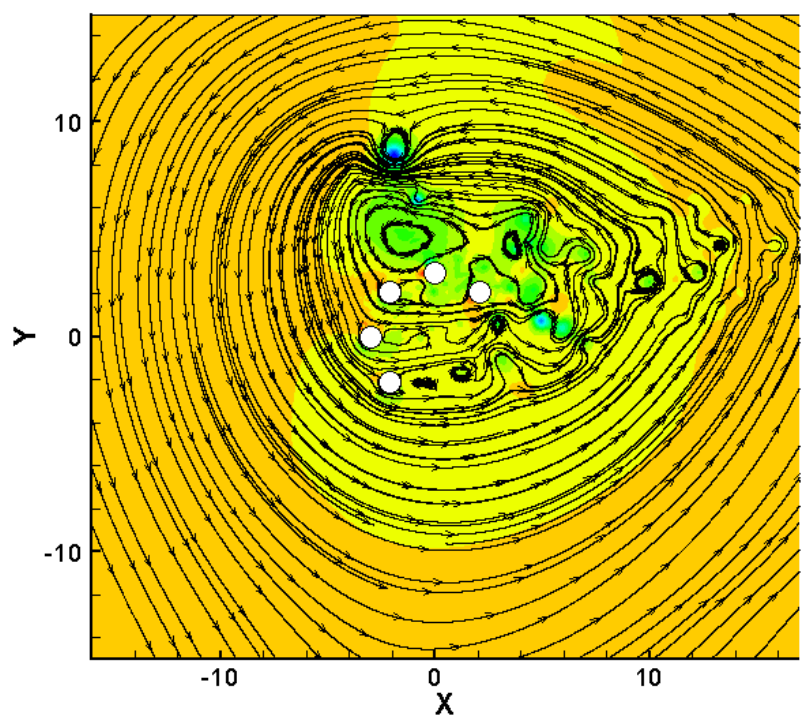

(b)

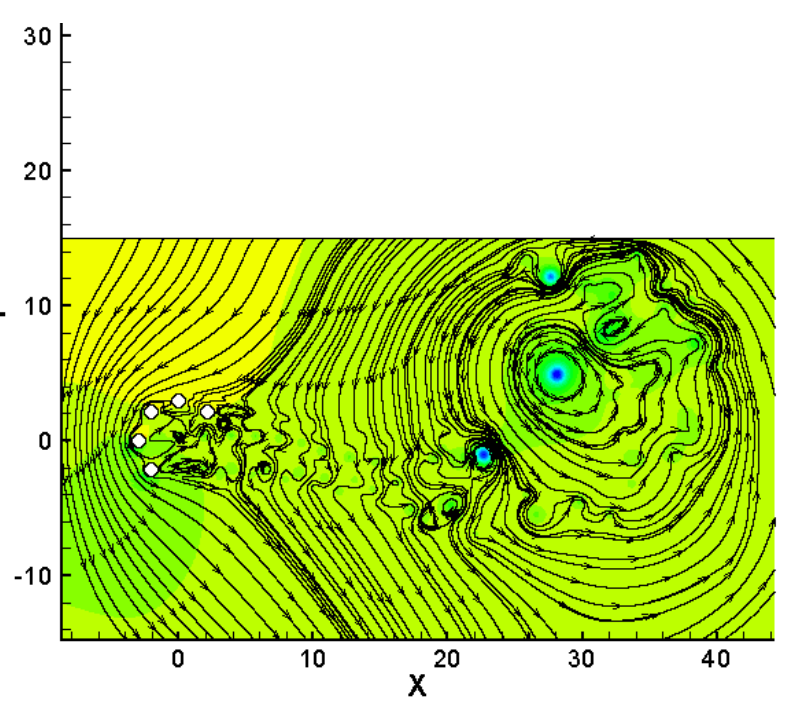

(d)

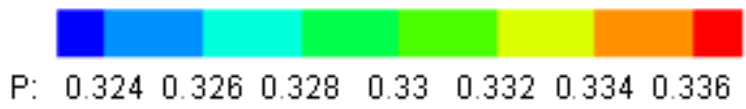

Fig. 4.18 Streamline contour for Case D at four representative time instants: (a).at right $r_{c}$; (b). tornado center; (c). at left $\mathrm{r}_{\mathrm{c}}$; (d). departed tornado) 


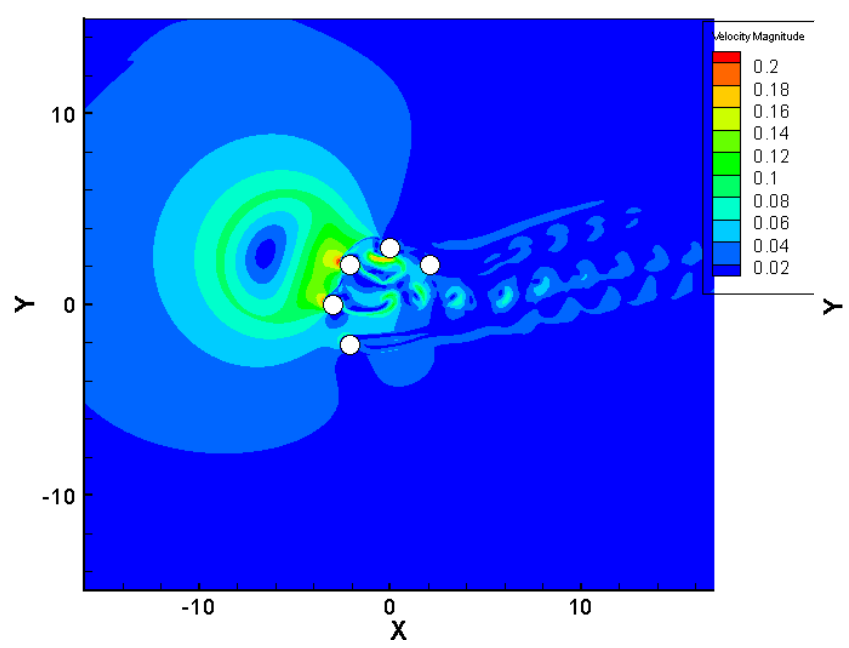

(a)

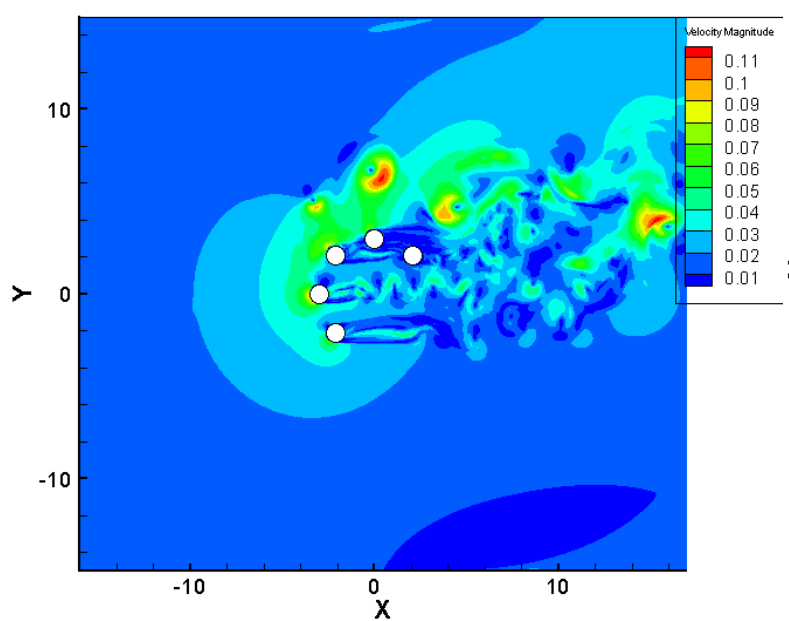

(c)

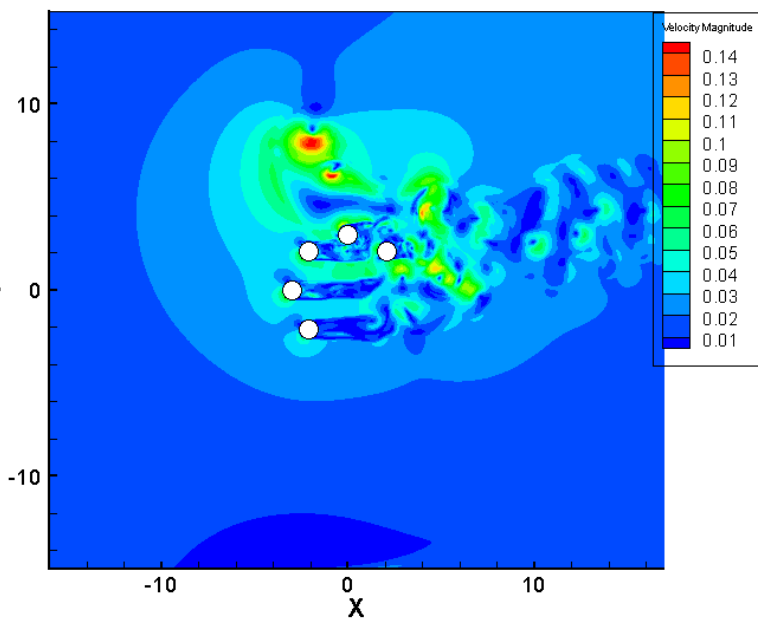

(b)

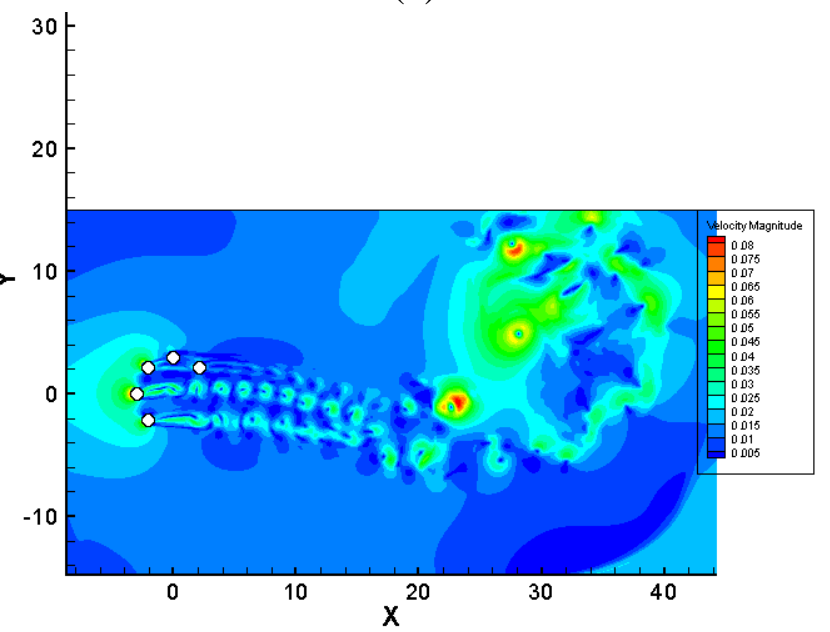

(d)

Fig. 4.19 Velocity magnitude for Case D at four representative time instants: (a).at right $r_{c}$; (b). tornado center; (c). at left $r_{c}$; (d). departed tornado) 


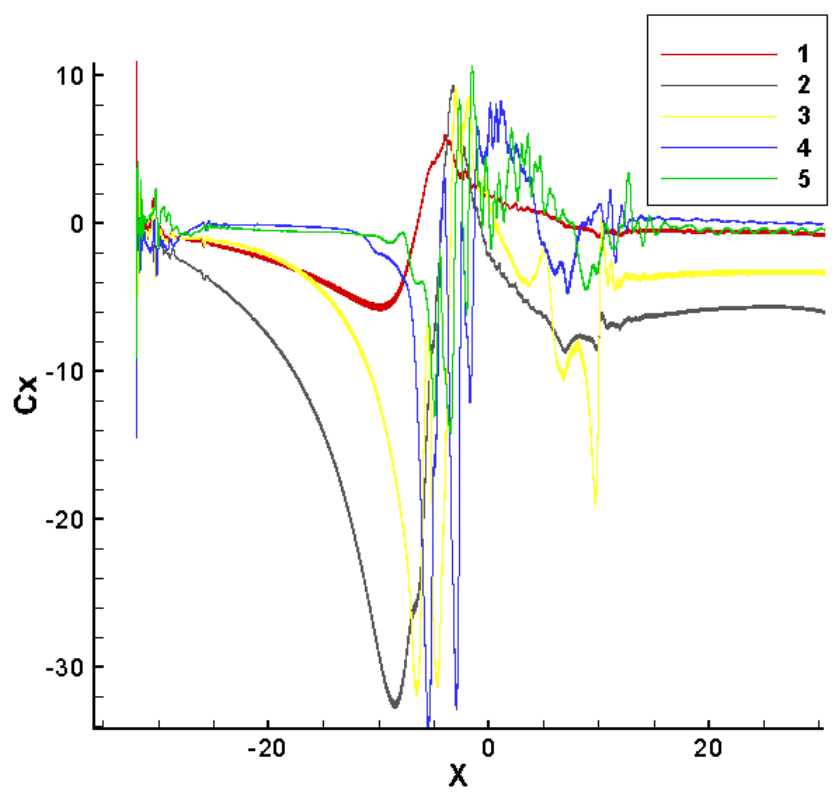

Fig. 4.20 X-direction force coefficient for Case D

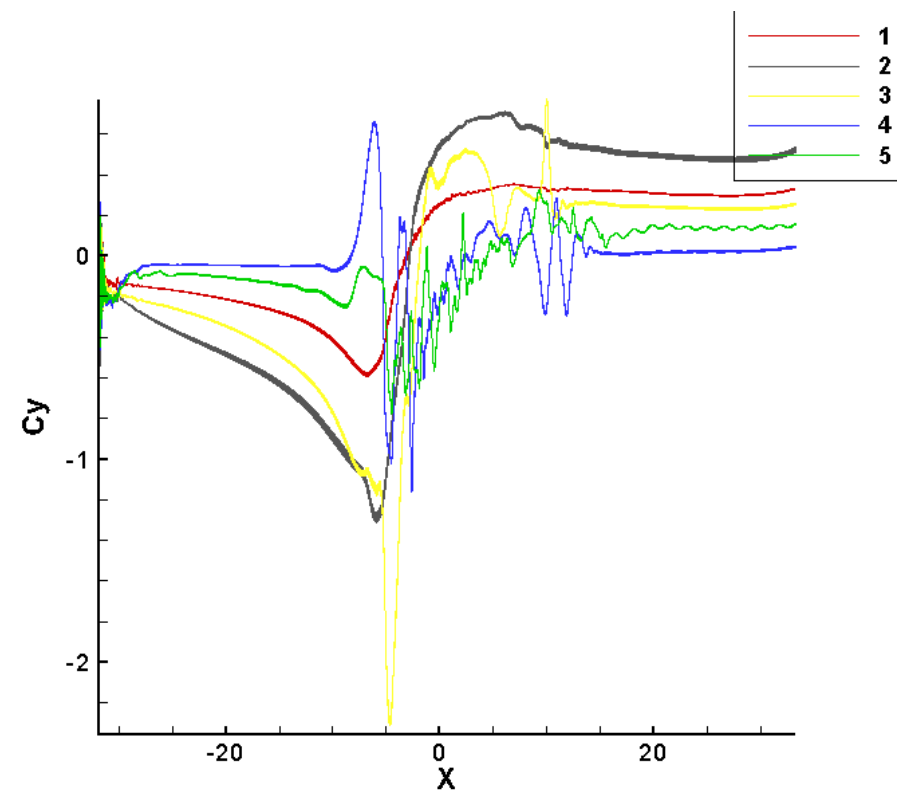

Fig. 4.21 Y-direction force coefficient for Case D 


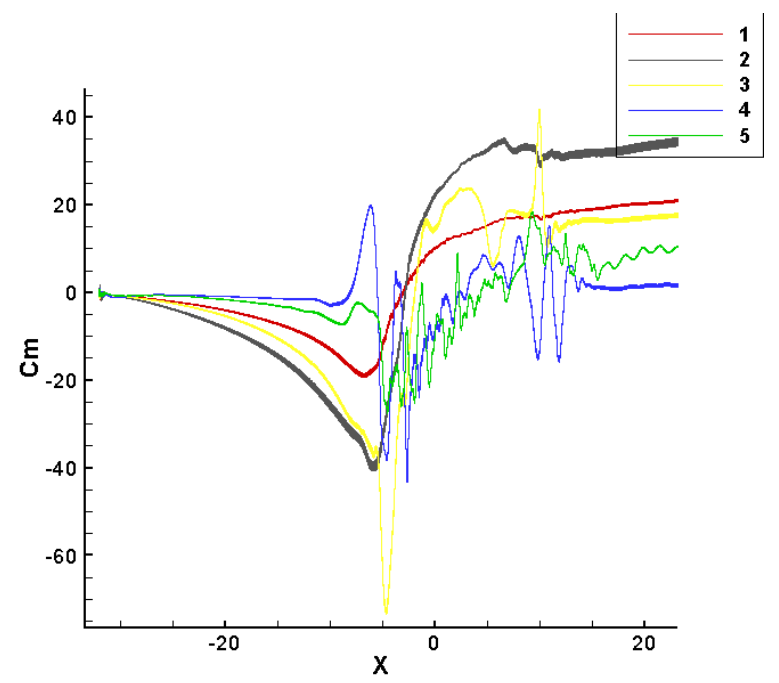

Fig. 4.22 Moment coefficient for Case D

\subsubsection{Case $\mathbf{E}$}

In this case, cylinder \#3 moves to the front of this configuration, and cylinder \#1 and \#5 are located at bottom and top of the cylinder-quintuplet configuration, respectively. Likewise, upward deviation can be observed for tornado center when the tornadic wind approaches the obstacles (see Fig. 4.23(a) and Fig. 4.24(a)). Again, taking the "deviation effect" and "front effect" into consideration, cylinder \#3, \#4 and \#5 suffer higher force loadings than the two cylinders located in lower part. The force in $x$-direction acting on cylinder \#5 is nearly two times higher than cylinder \#3 and \#4, however, $C_{y}$ and $C_{m}$ for cylinder \#4 are much larger than others (see Fig. 4.25 - Fig. 4.27).

Cylinder \#1 is located at the bottom, which is far away from the deviated tornado center, and three coefficients depicted in Fig. 4.25 - Fig. 4.27 indicate that rotational flow affects cylinder \#1 very limited, and the loading on this cylinder is close to 0 . Loadings exerted on cylinder \#2 are 
monitored to be higher than cylinder \#1, but still lower than the upward three cylinders apparently due to its position. Moreover, shedding vortices can be found in the wake region of the cylinder \#1 as depicted in Fig. 4.23(d) and Fig. 4.24(d), which again identifies the dominant effect of the translational flow when the cylinder-quintuplet goes distant.

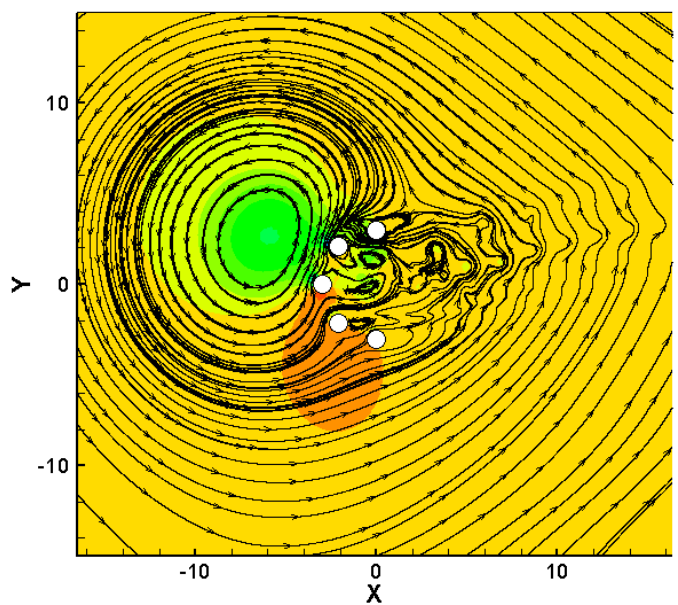

(a)

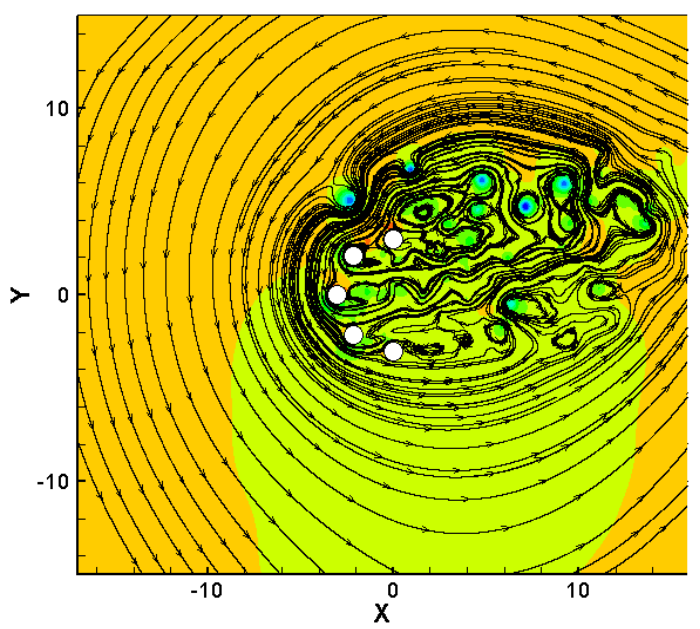

(c)

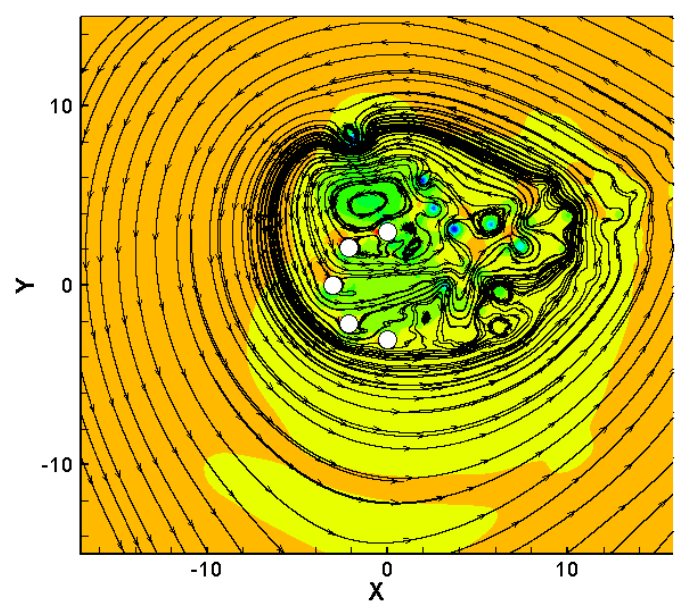

(b)

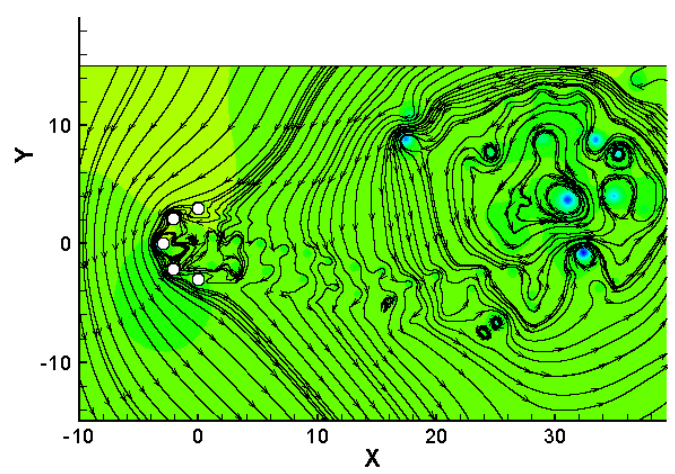

(d)

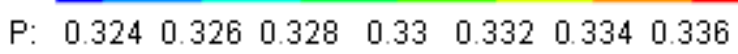

Fig. 4.23 Streamline contour for Case $\mathrm{E}$ at four representative time instants: (a).at right $\mathrm{r}_{\mathrm{c}}$; (b). tornado center; (c). at left $\mathrm{r}_{\mathrm{c}}$; (d). departed tornado) 


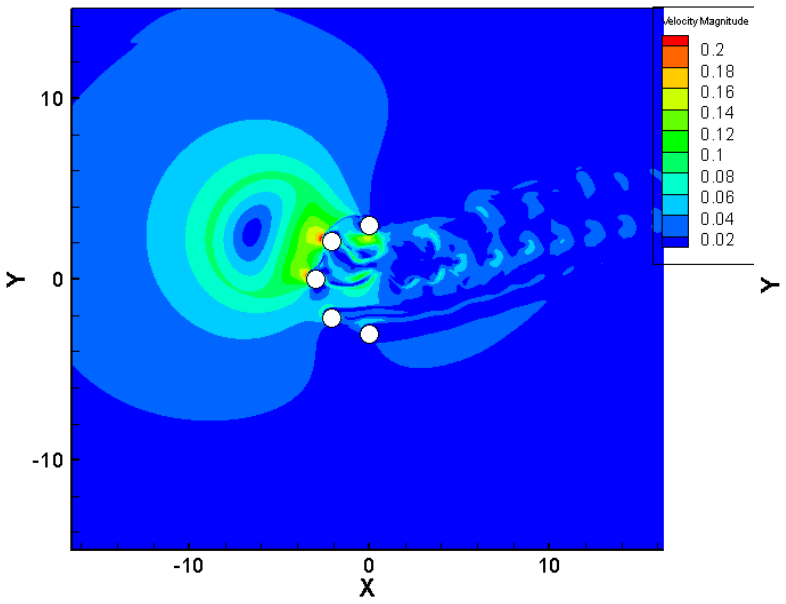

(a)

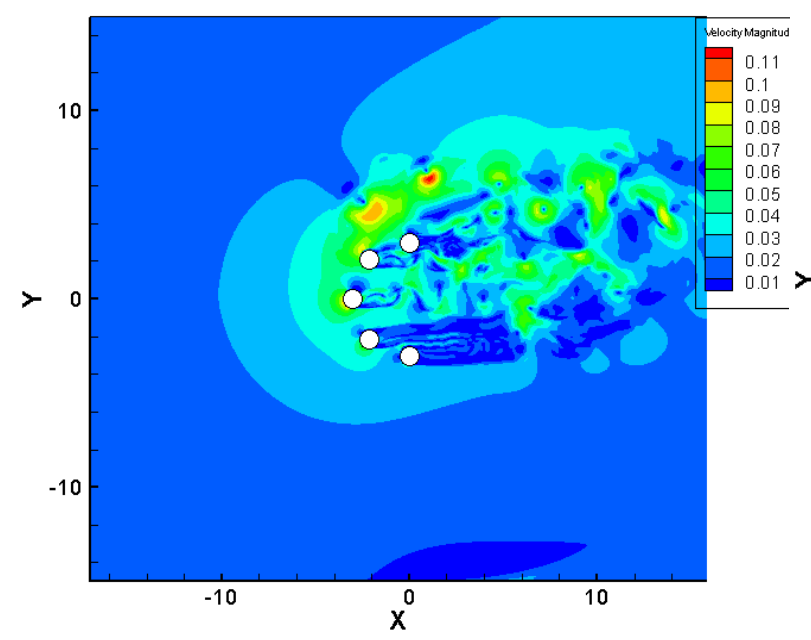

(c)

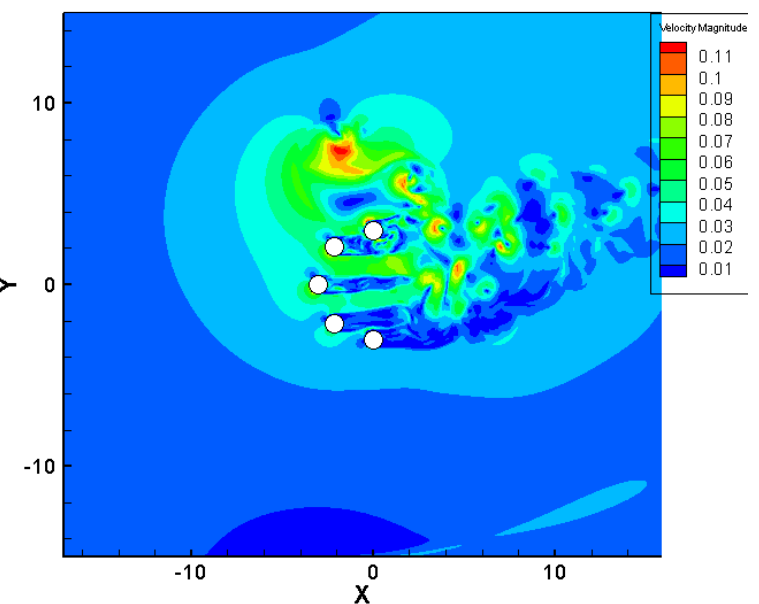

(b)

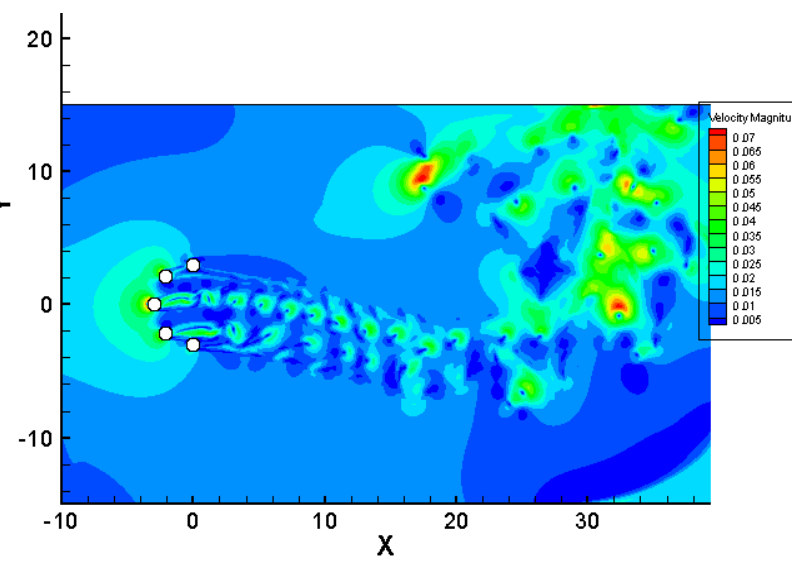

Fig. 4.24 Velocity magnitude for Case $\mathrm{E}$ at four representative time instants: (a).at right $\mathrm{r}_{\mathrm{c}}$; (b). tornado center; (c). at left $r_{c}$; (d). departed tornado) 


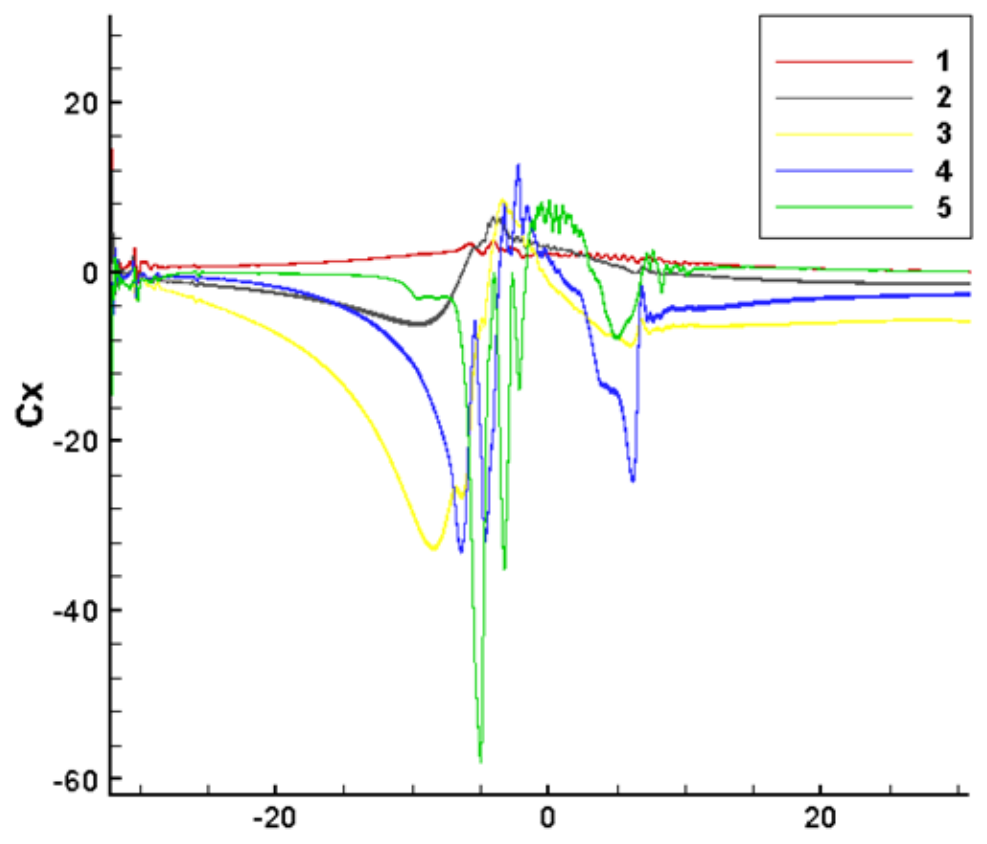

Fig. 4.25 X-direction force coefficient for Case E

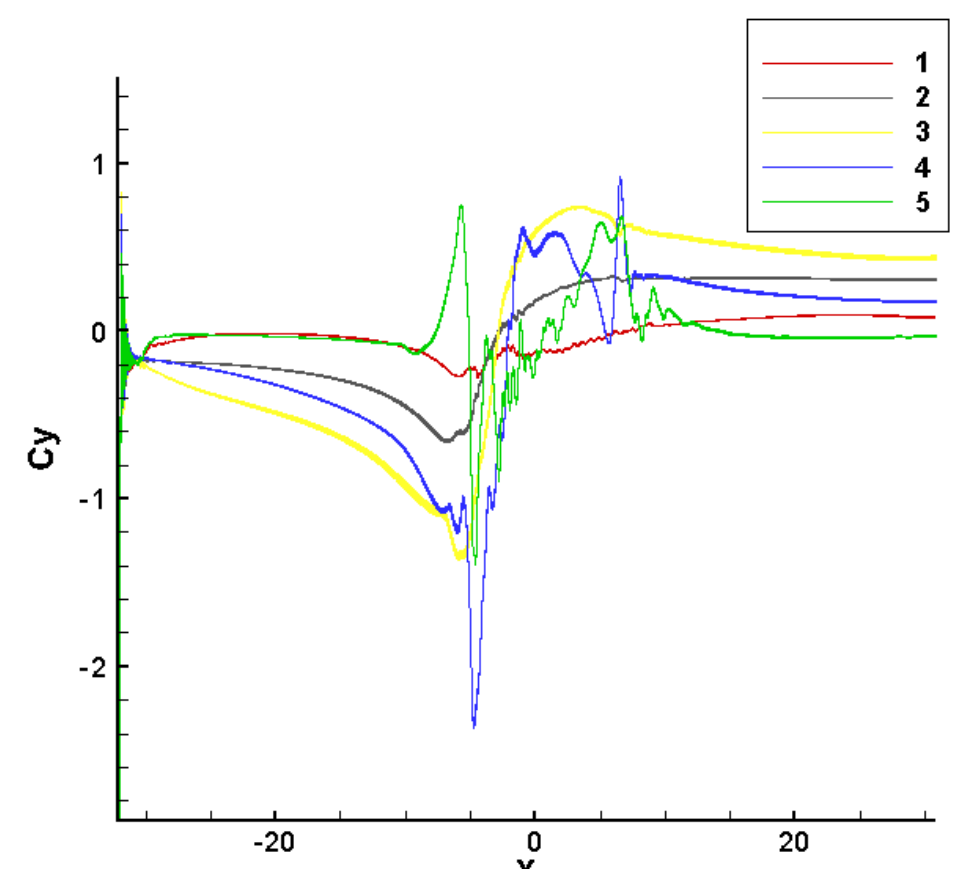

Fig. 4.26 Y-direction force coefficient for Case E 


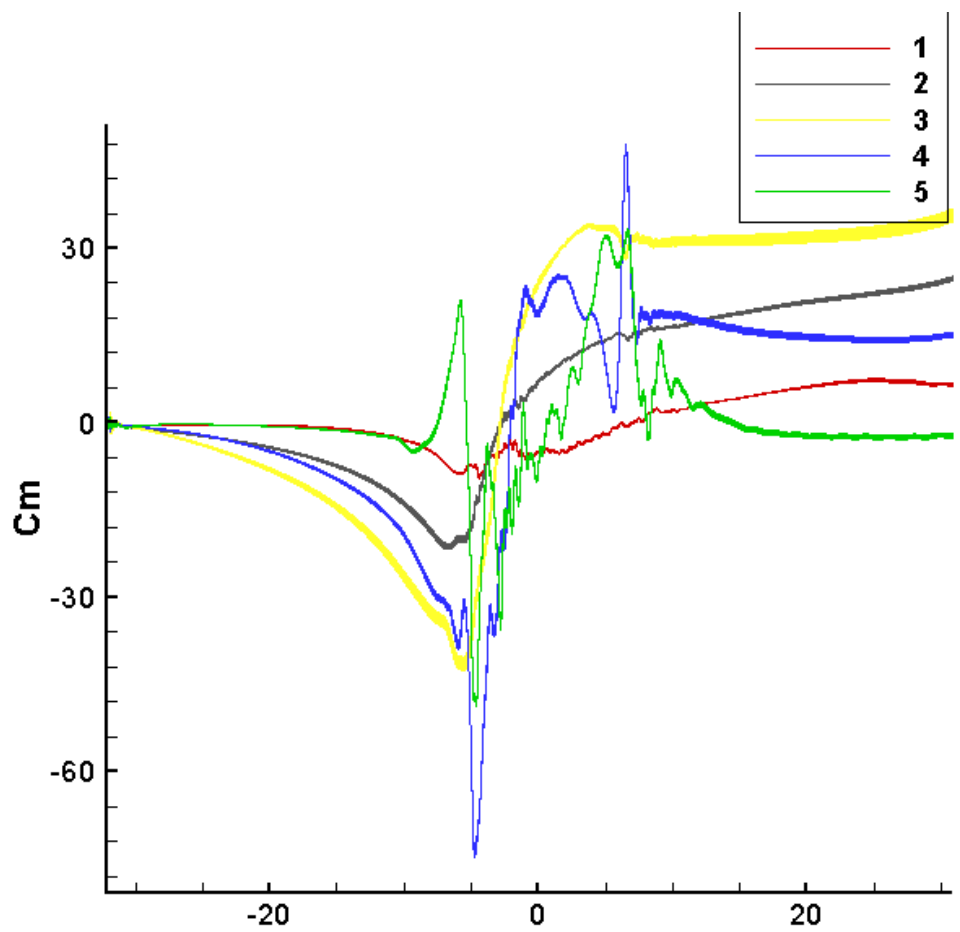

Fig. 4.27 Moment coefficient for Case E

\subsubsection{On rotation direction}

The tangential velocity $\vec{V}_{R}$ in precedent numerical cases are all based on Eq. (4.1), which is derived from the traditional RCVM. This governing equation leads to the counter-clockwise rotation direction when transforming into streamline contour. However, what if the rotation direction is reversed? Next, how rotation direction can affect the loadings will be investigated as follows.

When Eq. (4.1) is revised to:

$$
\vec{V}_{R}=V_{R}(\vec{i} \sin \theta-\vec{j} \cos \theta)
$$

So that clockwise circular-shaped streamlines are obtained as shown in Fig. 4.28, which can be 
easily observed to be contrary to the cases discussed in Section 4.3.1 - 4.3.5.

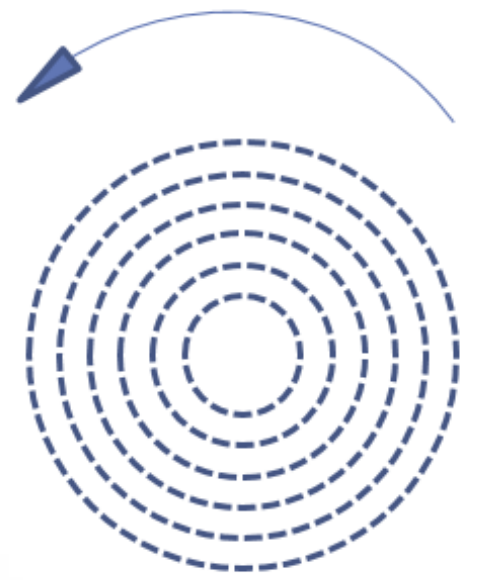

(a) Precedent cases in Section 4.3.1 - 4.3.5

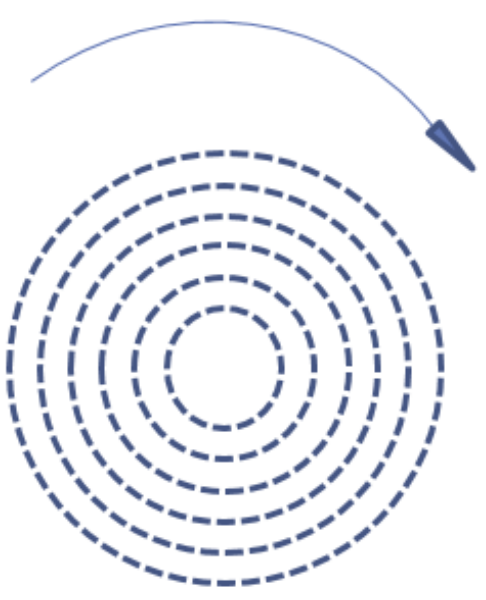

(b) new case in Section 4.3.6

Fig. 4.28 Comparison between precedent cases and current case on different rotation direction

Using the cylinder-quintuplet configuration in Case $A$ performed in Section 4.3 .1 as an example. All other variables remaining the same while changing the rotation direction by employing the tangential velocity as expressed in Eq. (4.2). Fig. 4.29 and Fig. 4.30 group the streamlines and velocity magnitudes at four representative time instants, respectively. Compared with counter-clockwise rotational case in Section 4.3.1 - 4.3.5, it is easy to observe that the "deviation effect" still exists, however, the tornado overall exhibit a downward trend (see Fig. 4.30(a)-(c)), instead of deviating to the upward as depicted in Fig. 4.3, and the vortex shedding phenomenon also presents downward trend in the wake region behind the cylinders. The velocity magnitude contours in Fig. 4.30(a-c) also illustrate that the velocities at the vicinity of cylinder \#5 and \#4 are larger than other three, in particular cylinder \#5, which indicates that cylinder \#5 is affected mostly attributed to 
the tornado-building interaction. Thus, according to the "front effect" and "deviation effect" aforementioned, the loadings exerted on cylinder \#5 are proved to the highest in this case, instead of that belonging to cylinder \#1 in Case A. Likewise, as cylinder \#4 located behind the cylinder \#5, the loading on cylinder \#4 is observed to be smaller than cylinder \#5, but still larger than other three cylinders located at upper half of the configuration. When the tornado moves farther from the cylinder group, the shedding of vortices can be observed in the upward direction (see Fig. 4.29(d)), which is opposite to the Case A in Section 4.3.1. The loading coefficients in Fig. 4.31 also confirms that the translational flow dominates the domain after the tornado passes the cylinders, especially cylinder \#1 and \#2 at upward of the configuration. As all the parameters remaining unchanged except for the rotation direction, the trend of each coefficient, as well as each extremum point, are all observed to be quite similar to the results obtained in Section 4.3.1. Only the sequence of the building loads, as well as the direction of $C_{y}, C_{m}$, reversed in current situation. 


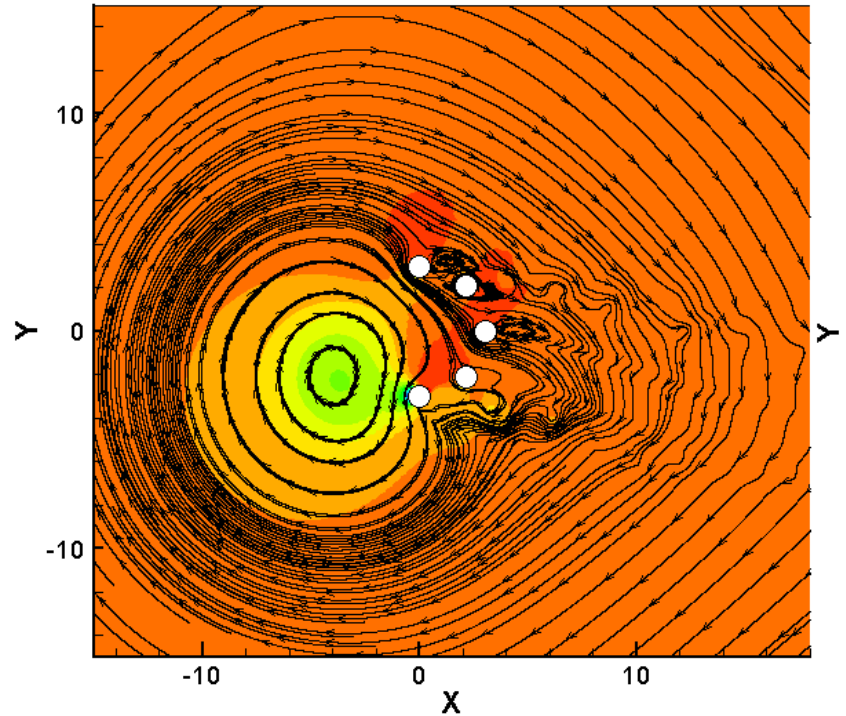

(a)

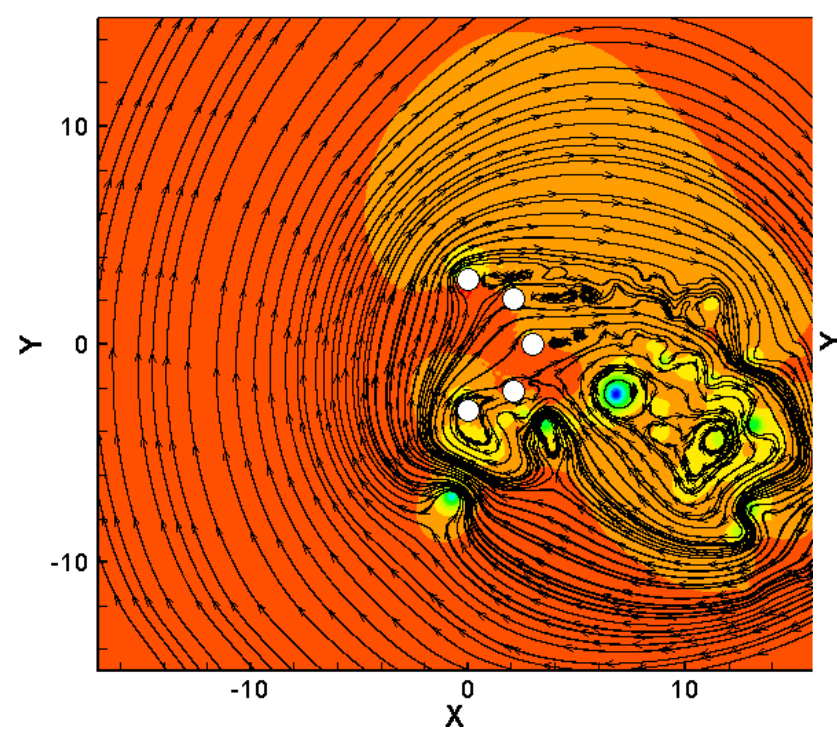

(c)

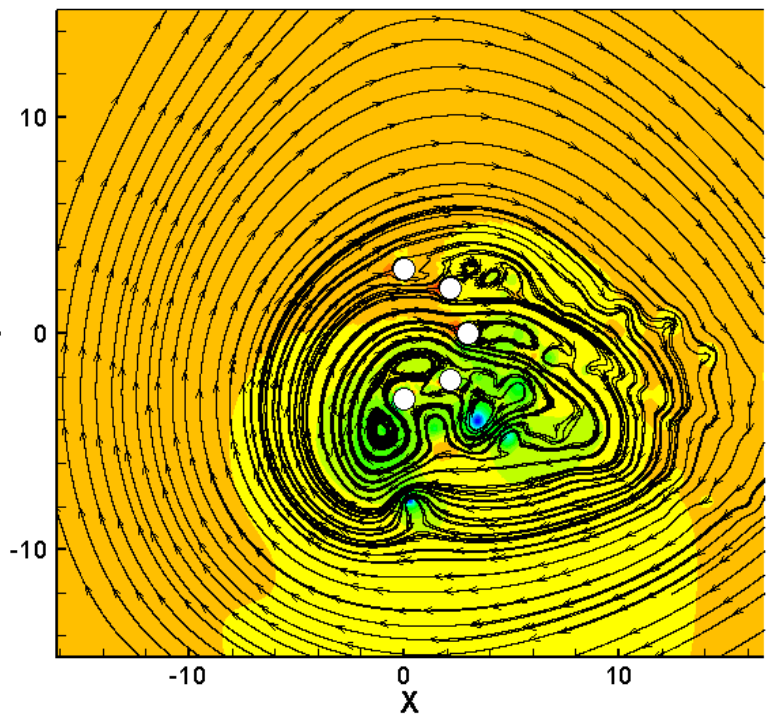

(b)

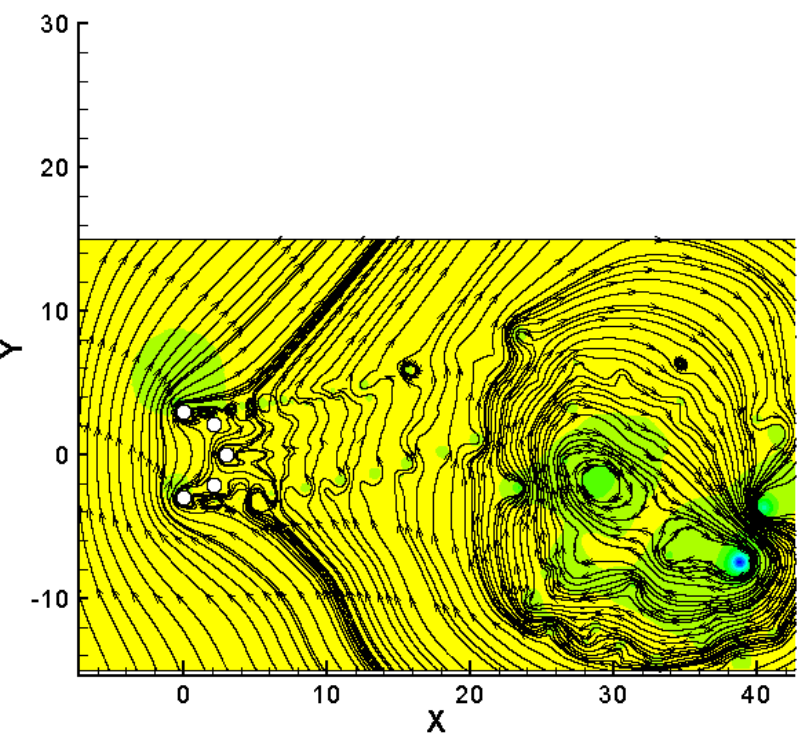

(d)

P: $\begin{array}{lllllll}0.324 & 0.326 & 0.328 & 0.33 & 0.332 & 0.334 & 0.336\end{array}$

Fig. 4.29 Streamline contour for clockwise rotation of tornadic wind at four representative time instants: (a).at right $r_{c}$; (b). tornado center; (c). at left $r_{c} ;(d)$. departed tornado) 


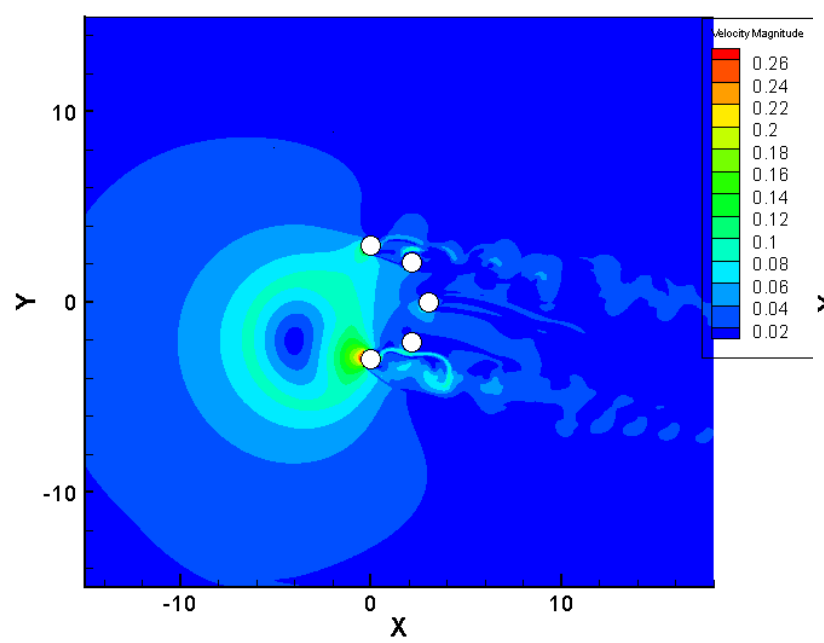

(a)

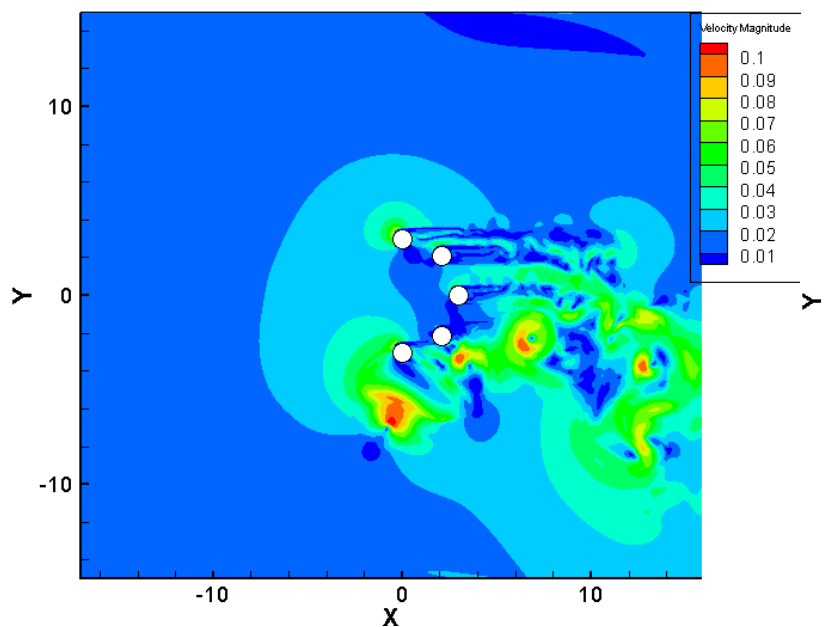

(c)

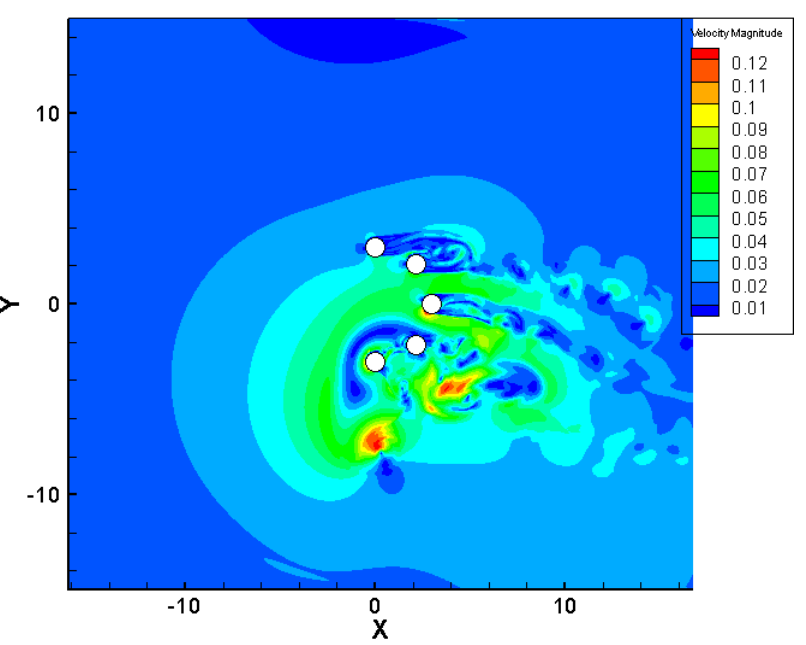

(b)

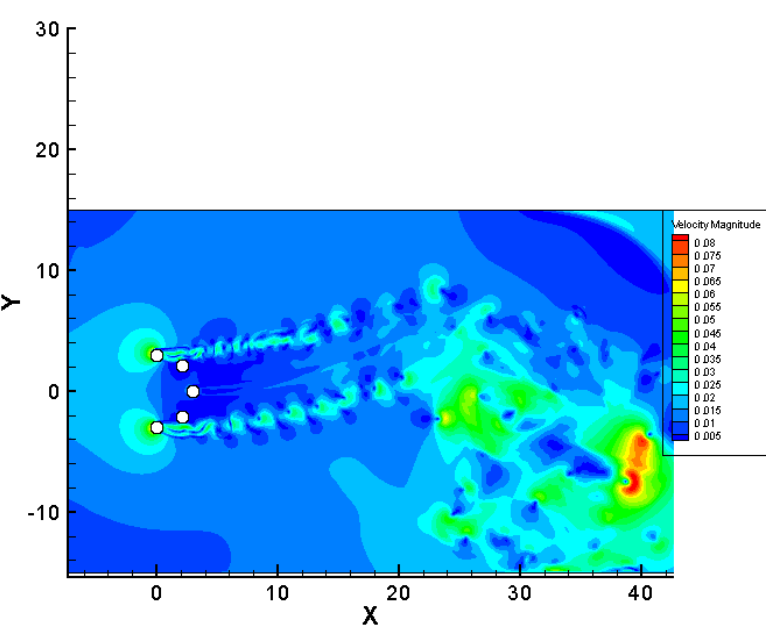

(d)

Fig. 4.30 Velocity magnitude for clockwise rotation of tornadic wind at four representative time instants: (a).at right $r_{c}$; (b). tornado center; (c). at left $r_{c}$; (d). departed tornado)

Rotation direction is proved to be a significant influencing factor when investigating the tornadic wind over obstacles in real world. The deviate direction can be determined after the rotation direction is identified, and the most dangerous region with largest loading can be determined at the same time. This significant discovery plays an important role for providing the civil designers a 
good understanding of the potential principle about tornadic wind loads distribution using numerical

way.
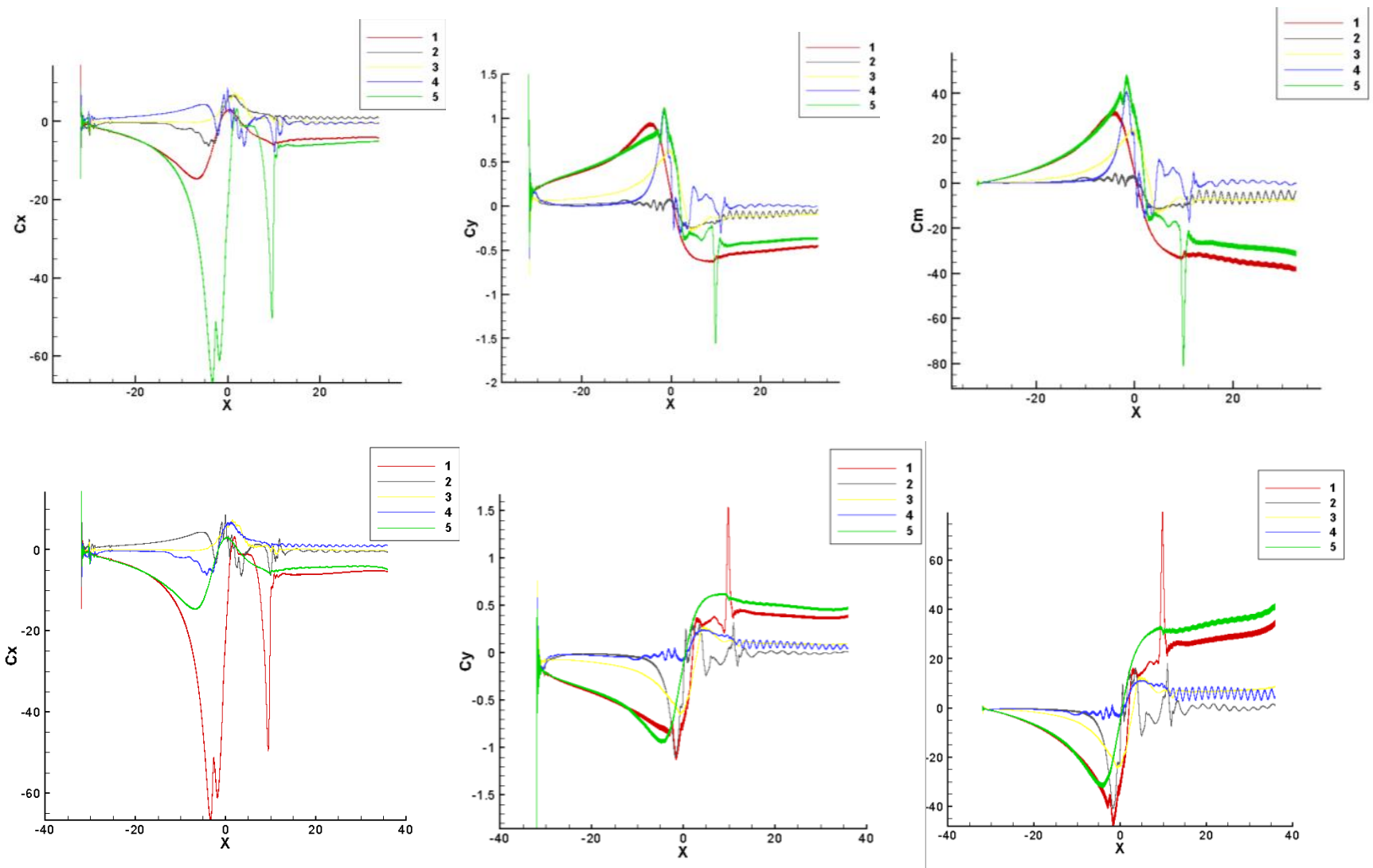

Fig. 4.31 Coefficients with different rotation direction

(Notes: first row represents the $\mathrm{C}_{\mathrm{x}}, \mathrm{C}_{\mathrm{y}}, \mathrm{C}_{\mathrm{m}}$ for clockwise rotation case; second row represents the $\mathrm{C}_{\mathrm{x}}, \mathrm{C}_{\mathrm{y}}, \mathrm{C}_{\mathrm{m}}$ for counter-clockwise rotation case.)

\subsection{Conclusions}

Extended from the Chapters 2 and 3, this paper focuses on its application to simulations of more practical problems in the case of tornadic wind load over cylinder-quintuplet configuration. A few distinctive features are demonstrated through various numerical experiments performed in the 
present study: 1) Tornadic wind tends to exhibit deviation trend depending on its rotation direction and the location of the obstacle. Despite its original trace, the tornado center is observed to vertically deviate upward or downward due to the interaction between the tornado and obstacles, which induces a much higher loadings exerting on obstacles located at the same side with the deviation direction than those located at opposite directions. Moreover, these obstacles reaching the tornado core earlier are monitored to endure a heavier loadings than those hiding aside the front obstacles. Inspired by these observations, namely "front effect" and "deviation effect", it is enlightening for the civil designers to plan several solid obstacles around the buildings group to resist the property damage induced by the tornado. The loadings on the building can be alleviated greatly when tornado first contact the solid obstructions. This design is regarded as an important seal for circumjacent buildings configuration. 2) Before "core-in" time instant, translating flow tends to dominate the wake of the obstacle, and this dominance reoccurs when the tornado goes distant. As implied in RCVM, the tangential velocity outside the inner core of tornado is decreased steeply along the radial direction, which results the dominance of translating flow reflected on the flow pattern. Loadings at the beginning of "pre-interaction" stage and "post-interaction" stage are relatively slight with smaller slope, in particular loading in $y$-direction. Moreover, vortex shedding phenomenon can be detected at the wake of the obstacles after the "core-out" time instant, which further manifests the dominance of translating flow at the end stage of the simulation. 3) Tornadic wind loadings vary tremendously in "primary" stage. As illustrated in the coefficient evolutions in Section 4.3, the 109 
loadings undergo an intensive variation during the "primary" stage, and the maximum value can reach ten times as high as that in translating-dominated time instant. This indicates the significant impact attributed to the rotating feature of tornado, and the huge magnitude of the loadings also manifest the reason why the disastrous wind causes the numerous property damages. 


\section{Chapter 5 A Novel Three-Dimensional Immersed-Boundary (IB) Approach Based Tornado Model Powered by Prediction-Correction Technique Part I - Numerical Methods and Benchmark Testing}

This chapter is based on the following paper (ready to submit to Journal of Computational Physics): Xixiong Guo, Jun Cao. A Novel Three-Dimensional Immersed-Boundary (IB) Approach Based Tornado Model Powered by Prediction-Correction Technique Part I - Numerical Methods and Benchmark Testing

\subsection{Summary}

Focusing on the study of three-dimensional tornado dynamics, a novel computer simulation framework has been built that features conjoining multiple numerical techniques in a seamless manner. Due to the large scope of this study, its presentation unfolds in two parts. This first part mainly address the numerical methods employed in this study, and the validation with the aid of a series of benchmark cases. The incorporated numerical methods mainly include the immersed boundary method (IBM), high-order compact finite difference scheme, and prediction-correction strategy. In order to more efficiently deal with moving boundary problems, a three-dimensional hybrid Navier-Stokes flow model based solver is developed in this study that embeds a feedback forcing based immersed boundary method into a high-order compact finite difference discretization 
of space. Moreover, for the sake of numerical accuracy improvement and convergence acceleration, a prediction-correction scheme is employed, such that the feedback forcing term in IBM experiences both prediction and correction in each time-advancing step, and the simulation results reveal that the convergence can be accelerated by over $40 \%$ along with enhanced numerical accuracy owing to the prediction-correction technique. This highly hybrid computational framework has been validated by a large set of numerical experiments, with its advantages clearly demonstrated.

\subsection{Introduction}

The greatest bottleneck of tornado investigation using numerical methods is its highly dynamic nature. The velocity boundary condition is time-dependent when taking into account all its translational, rotational, and vertical components. Conventional methods to deal with moving boundary problems, such as Arbitrary Lagrangian Eulerian (ALE) method [61]-[62] and fictitious-domain methods [63]-[64], need to employ body-fitted grids that conform to the geometry of the solid surface for discretization of the governing equations. The time-dependent boundary needs to be updated, and the structure of the body-fitted mesh needs to be reconstructed every time step in accordance with the moving object, which is computationally very painful and, in turn, costly.

To overcome that tedious updating process, Guo et al. [65]-[66] examined the tornado-induced loadings with the aid of immersed boundary method (IBM) and "relative motion" principle. The IBM is a simple, but efficient tool applied to the interaction problems between fluid and structures 
with arbitrary boundaries using a Cartesian-style grid. That is, the computational grid does not need to conform to the boundaries of solid objects no matter how the objects move or deform, with the desired boundary conditions imposed by applying a numerical algorithm in the vicinity of the immersed boundary. Therefore, IBM effectively avoids the difficulties faced in the grid regeneration process when dealing with flow associated with complex geometries. In addition, considering the "relative motion" strategy, a "virtual" translation of the building towards a rotating airflow about its "virtually locked" center was conducted. Thus, a time-independence form of boundary condition was set up, and the original highly time-dependent model can be completely converted as a traditional moving boundary problem, which was solved with IBM efficiently and straightforwardly.

In previous studies (Chapters 2-4), the tornado-induced loading on a single cylinder-shaped building and configurations of such buildings were extensively studied and, particularly, the relation between the rotation intensity of a tornado and the loadings on the constructions was investigated. However, this series of studies focused on a two-dimensional (2D) scenario, which represent the flow behavior on a horizontal plane with a cross-section of the building. Tornadoes, well-known as three-dimension (3D) flow phenomenon, fundamentally devastate the building structures with the combination of simultaneous velocity components in all three directions [67]-[68]. In order to achieve a better understanding of the underlying loadings induced by tornadoes with all directions considered, especially in the vertical z-direction, this study aims to extend the tornado simulation to all three dimensions. 
The excessive computational cost required for 3D tornado dynamics study is the most challenging barrier, as the entire computational domain required for 3D simulation increases exponentially if compared with previous $2 \mathrm{D}$ simulations [65]. Thus, it is essential to employ a powerful computational framework with massive and high efficient parallelizability, which is also eligible to deal with fluid-structure interaction (FSI) problems.

Incompact3d [69]-[70] is an efficient numerical tool that can be coupled with massive parallel platforms in order to simulate turbulence problems, using large eddy simulation (LES) [71] or direct numerical simulation (DNS) [72] strategies. Incompact3d is based on Cartesian mesh. The use of such a simplified mesh offers the opportunity to implement high-order compact schemes for the spatial discretization. Furthermore, an immersed boundary method (IBM) is also seamlessly embedded into the package, which allows the implementation of any solid body, complex geometry or moving boundary problems inside the computational domain. With all these features enabled, this open source package can be utilized for 3D tornado-construction interaction study as long as specific modifications are made.

In spite of the aforementioned advantages of Incompact3d, its fatal drawback lies in the insufficient accuracy due to its direct forcing based IBM. Historically, IBM was proposed by Peskin [30] in order to study the blood flow in human heart a few decades ago, following the work of Peskin [30], intensive effort in the IB area can be categorized by the mechanism used to build the restoring force. Mohd-Yusof [73] and Fadlun et al. [74] recast the immersed boundary method based 
on a direct forcing approach. A key to this method is the enforcement of boundary conditions directly, through specification of local distributions of the fluid properties (velocity and sometimes pressure) near the immersed boundary. Wu et al. [60] presented an implicit scheme named velocity-correction method, in which the restoring force is no longer pre-calculated and keeps updated by introducing a velocity-correction step to constantly ensure the satisfaction of non-slip condition. Yang and Balaras [75] developed a variance of the embedded-boundary approach with the aid of multi-dimensional interpolation to interpret the grid-interface relation for complex boundary shapes. Taira and Colonius [76] proposed the immersed-boundary projection method, in which the pressure and restoring forces are treated together as Lagrangian multipliers, and solved by fractional step (projection) method. Goldstein et al. [41] introduced a feedback forcing approach to enforce the desired boundary conditions at the immersed boundary. This feedback forcing was successfully applied to many moving boundary problems with satisfactory accuracy. On the other hand, a number of recent studies focused on incorporating immersed-boundary methods into high-order CFD solvers. For example, Zeng et al. [77] used a third-order upwind-biased finite-difference scheme along with a ghost-cell based immersed-boundary method to solve 1D and 2D transport problems, which resulted in an overall third-order accuracy. Seo et al. [78] combined the high-order compact finite-difference scheme and a sharp-interface based immersed-boundary method to solve aeroacoustic problems with complex bodies, and they interpreted the relation between fluid points and discretized solid boundary points by high-order polynomial interpolation. 
Yang et al. [79] managed to solve the moving boundaries on laminar and transitional flow by solving the filtered incompressible Navier-Stokes equations using IBM and large eddy simulation techniques. Linnick and Fasel [80] employed an explicit fourth-order Runge-Kutta time integration scheme, fourth-order compact finite-differences compact method to solve two-dimensional incompressible Navier-Stokes equations in the stream function-vorticity formulation, and a fourth-order immersed-boundary method was applied to treat the boundary at the same time.

The IBM implementation in the original Incompact3D code relies on combination of direct forcing approach [81] with centered finite difference schemes of high accuracy. This combination is a priori problematic due to the discontinuities on velocity and pressure field at the vicinity of the immersed boundary, especially when the solid object is in moving condition. As a straightforward and explicit solver, feedback forcing model [41] has been proven to be a more accurate and efficient IB approach. In this study, the feedback forcing IB model is chosen to be incorporated into the Incompact3d package, making the first time of coupling the feedback forcing based IB with high-order finite difference discretization scheme. Moreover, a prediction-correction strategy is also first time adopted into IB module in order to achieve faster convergence and better accuracy.

The rest of this paper will unfold as follows. Section 5.3 describes the details of proposed feedback forcing based framework for this study, followed by series of benchmark testing cases for validation purpose, as demonstrated in Section 5.4, both in stationary and moving status. Finally, some concluding remarks are made in Section 5.5 for the first stage of this study. Extended 
simulations focusing on the study of tornado dynamics will be presented in the second part that will follow.

\subsection{Numerical methods}

For modeling a viscous incompressible fluid flow with an object immersed in the fluid, the Navier-Stokes (N-S) equations with external force term enabled have been routinely employed. Furthermore, an immersed-boundary (IB) method based on feedback forcing model [41], which has been explained in Section 2.4.2, is incorporated in the $\mathrm{N}-\mathrm{S}$ flow model, aiming to solve the fluid-solid interaction (FSI) problems. Details of this IB-embedded N-S framework will be described in this section.

\subsubsection{Governing equations}

Mass and momentum conservation principles are represented by the Navier-Stokes equations, which have the following form for an incompressible fluid

$$
\begin{gathered}
\rho\left(\frac{\partial \vec{u}}{\partial t}+\vec{u} \cdot \nabla \vec{u}\right)=-\nabla p+\mu \Delta \vec{u}+\overrightarrow{\mathrm{f}} \\
\nabla \cdot \vec{u}=0
\end{gathered}
$$

where $\vec{u}(\vec{x}, t)=(u(\vec{x}, t), v(\vec{x}, t), w(\vec{x}, t))$ is the fluid velocity, $p(\vec{x}, t)$ is the pressure field, and $\rho$ is a constant density $(\rho=1)$ in the present framework. $\mu$ is the dynamic viscosity, and $\overrightarrow{\mathrm{f}}$ is the external forcing term caused by the interaction between immersed object and fluid, which can be 
obtained from the immersed boundary method. The equations are spatially discretized and solved by using sixth-order, compact finite-different schemes, and details of the solution procedure are described in the subsequent sections.

\subsubsection{High-order finite difference discretization}

In order to numerically solve the governing equations, Incompact3d [69], an open-source code developed mainly by Laizet \& Lamballais [69] and Laizet and Li [81] from Université de Poitiers and Imperial College London, respectively, was applied as the primary N-S solver. All governing equations are discretized and solved on a collocated velocity grid via the six-order central compact finite different scheme [82], while the pressure is on a staggered grid. A second-order Adams-Bashforth (AB) discretization scheme is used for time-advancement, which will be detailed in the next subsection. The continuity is verified at the end of each sub-time step by solving a pressure Poisson equation. This Poisson equation is fully solved through a spectral solver via the utility of 3D Fourier transforms to avoid the expensive cost incurred by the application of high-order scheme combined with iterative techniques. The code allows for massively parallel processing through MPI implementation, which is built upon the pencil domain decomposition strategy [81].

\subsubsection{Time discretization}

The time integration for solving Eq. (5.1) is performed using a second-order Adams-Bashforth 
scheme. A fractional step method [83]-[86] is applied to deal with the pressure and the incompressibility constraint by decoupling the velocity and pressure through the following successive steps:

$$
\begin{gathered}
\frac{\vec{u}^{*}-\vec{u}^{n}}{\Delta t}=\frac{3}{2} \vec{N}^{n}-\frac{1}{2} \vec{N}^{n-1}-\nabla \tilde{p}^{n}+\tilde{\overrightarrow{\mathrm{f}}}^{n+1} \\
\frac{\vec{u}^{* *}-\vec{u}^{*}}{\Delta t}=\nabla \tilde{p}^{n} \\
\Delta \tilde{p}^{n+1}=\frac{\nabla \cdot \vec{u}^{* *}}{\Delta t} \\
\frac{\vec{u}^{n+1}-\vec{u}^{* *}}{\Delta t}=-\nabla \tilde{p}^{n+1}
\end{gathered}
$$

with

$$
\vec{N}^{n}=-\vec{u}^{n} \cdot \nabla \vec{u}^{n}+\mu \Delta \vec{u}^{n}
$$

and

$$
\tilde{p}^{n+1}=\frac{1}{\Delta t} \int_{t_{n}}^{t_{n+1}} p d t, \quad \tilde{\overrightarrow{\mathrm{f}}}^{n+1}=\frac{1}{\Delta t} \int_{t_{n}}^{t_{n+1}} \overrightarrow{\mathrm{f}} d t
$$

where $\vec{u}^{* *}$ and $\vec{u}^{*}$ are the intermediate velocities obtained between time step $(\mathrm{n}+1)$ and $\mathrm{n}$. Remark that, in order to solve the Eq. (5.3), the forcing term $\tilde{\overrightarrow{\mathrm{f}}}^{n+1}$ should be pre-determined to satisfy the boundary condition of the immersed object, and this term, will be described in the next sub-section 
where the immersed boundary method is elucidated.

\subsubsection{Forcing term calculation}

In order to model the interaction between the immersed object and the fluid, an external forcing term is added to the governing equation, which is already depicted in Eq. (5.1). IBM (see Section 2.4.2) is applied to calculate the forcing term $\overrightarrow{\mathrm{f}}$. In order to deal with the numerical oscillation that is often present in the simulation of moving boundary problems, the feedback forcing model is applied in the this study, instead of using the direct forcing method that was embedded in the original Incompact3d package, so that the accuracy of the numerical solution of velocity at the vicinity of the immersed obstacle can get significantly improved, especially when the immersed object is moving. The forcing term can be determined as follows [41]:

$$
\begin{gathered}
\vec{u}(\vec{X}(s, t), t)=\int_{\Omega} \vec{u}(\vec{x}, t) \delta(\vec{x}-\vec{X}(s, t)) d \vec{x} \\
\vec{U}_{B}(\vec{X}(s, t), t)=\vec{u}(\vec{X}(s, t), t) \\
\vec{F}_{R S T}=\alpha_{1} \int_{0}^{t}\left(\vec{U}_{B}-\vec{U}_{0}\right) d t+\alpha_{2}\left(\vec{U}_{B}-\vec{U}_{0}\right) \\
\vec{f}_{R S T}(\vec{x}, t)=\int_{\Gamma} \vec{F}_{R S T}(s, t) \delta(\vec{x}-\vec{X}(s, t)) d s
\end{gathered}
$$

where $\vec{x}$ and $\vec{u}$ are the Eulerian coordinates and fluid velocity; $\vec{f}_{R S T}$ represents the force density acting on the fluid due to the immersed boundary, which is also referred to as "restoring force"; $s$ represents the Lagrangian parametric coordinates; $\vec{X}$ and $\vec{U}_{B}$ are the coordinates and velocity of 
the Lagrangian points, $\vec{U}_{0}$ is the velocity of the immersed object; $\vec{F}_{R S T}$ is the density of the boundary force strictly distributed along the immersed boundary, which is also referred to as "Lagrangian force"; and $\delta(\vec{x}-\vec{X}(s, t))$ is the Dirac delta function.

In an incompressible viscous fluid flow field $\Omega$, according to Eqs. (5.9) and (5.10), the surface curve of immersed obstacle is discretized into $m$ Lagrangian points, and the discretized form of the velocity at a Lagrangian point denoted by the superscript $l(l=1,2,3, \ldots, m)$ can be expressed as:

$$
\vec{U}_{B}^{l}\left(\vec{X}_{B}^{l}, t\right)=\sum_{i, j, k} \vec{u}(\vec{x}, t) D_{i j k}\left(\vec{x}_{i j k}-\vec{X}_{B}^{l}\right) \Delta x \Delta y \Delta z
$$

in which $D_{i j}\left(\vec{x}_{i j}-\vec{X}_{B}^{l}\right)$, as replacement of the Dirac function $\delta(\vec{x}-\vec{X}(s, t))$ in Eq. (5.9) for measuring the closeness of an Eulerian point and a Lagrangian point, takes the following approximation:

$$
D_{i j k}\left(\vec{x}_{i j k}-\vec{X}_{B}^{l}\right)=\delta\left(x_{i j k}-X_{B}^{l}\right) \delta\left(y_{i j k}-Y_{B}^{l}\right) \delta\left(z_{i j k}-Z_{B}^{l}\right)
$$

where $\delta(r)$ is a continuous function defined as follows for all real $r$ :

$$
\delta(r)= \begin{cases}\frac{1}{4 h}\left(1+\cos \left(\frac{\pi|r|}{2 h}\right)\right), & |r| \leq 2 h \\ 0, & |r|>2 h\end{cases}
$$

which was originally proposed by Peskin [31], and $h$ represents the mesh spacing to perform the delta function interpolation. After velocity at the Lagrangian point is obtained via Eq. (5.9), no-slip boundary condition should be satisfied strictly, which is guaranteed in Eq. (5.10). Next, the restoring force per unit volume exerted on a Lagrangian point can be calculated using Eq. (5.11). A 
discretized version of Eq. (5.11) at time step $j=n$ can be expressed as:

$$
\vec{F}_{R S T}=\alpha_{1} \sum_{j=1}^{n}\left(\left(\vec{U}_{B}\right)_{j}-\left(\vec{U}_{0}\right)_{j}\right) \Delta t+\alpha_{2}\left(\left(\vec{U}_{B}\right)_{j}-\left(\vec{U}_{0}\right)_{j}\right)
$$

According to [41], $\alpha_{1}$ and $\alpha_{2}$ are stable for moderate values within the interval [-100, -1], and neither is sensitive to its nominal exact value.

Next, the force density $\vec{f}_{R S T}$ at Eulerian points can be calculated once the $\vec{F}_{R S T}$ is available, as shown in Eq. (5.12). Then, $\vec{f}_{R S T}$ is embedded into the governing equation as an external force. Thus, the effect induced by the interaction between the solid object and fluid is alternatively considered.

\subsubsection{Embedment of prediction-correction strategy into IB}

Inspired by the advantage in accelerating numerical convergence, a prediction-correction scheme [87] is employed in this feedback force model, aiming to speed up the numerical calculation and enhance the numerical accuracy during IB process. The method has been widely used in nonlinear partial differential equations (PDEs), but has not been found to collaborate with IBM yet. The idea of prediction-correction method is the combination of an explicit and an implicit technique to obtain a method with better convergence characteristics. The algorithm is normally divided into two steps: an initial predicted value obtained from its original value in the prediction step, and an improvement of initial predicted value is conducted using trapezoidal rule in the correction step. This embedment made in this study represents another significant improvement of the original 
Incompact3d package, in addition to the aforementioned feedback forcing based IBM implementation.

When utilizing into feedback forcing based IB algorithm, combining Eqs. (5.3) and (5.4), the governing equation is turned to:

$$
\frac{\vec{u}^{* *}-\vec{u}^{n}}{\Delta t}=\frac{3}{2} \vec{N}^{n}-\frac{1}{2} \vec{N}^{n-1}+\frac{1}{\Delta t} \int_{t_{n}}^{t_{n+1}} \overrightarrow{\mathrm{f}} \mathrm{dt}
$$

with $\overrightarrow{\mathrm{f}}=f(\vec{u}, t)$. According to the principles of prediction-correction algorithm [36], firstly, compute a predicted $\left(\vec{u}^{* *}\right)^{I}$ through:

$$
\frac{\left(\vec{u}^{* *}\right)^{I}-\vec{u}^{n}}{\Delta t}=\frac{3}{2} \vec{N}^{n}-\frac{1}{2} \vec{N}^{n-1}+f\left(\vec{u}^{n}, t\right)
$$

Then, a corrected solution for $\vec{u}^{* *}$ based on predicted $\left(\vec{u}^{* *}\right)^{I}$ is obtained by solving:

$$
\frac{\vec{u}^{* *}-\vec{u}^{n}}{\Delta t}=\frac{3}{2} \vec{N}^{n}-\frac{1}{2} \vec{N}^{n-1}+\frac{1}{2}\left[f\left(\vec{u}^{n}, t\right)+f\left(\left(\vec{u}^{* *}\right)^{I}, t\right)\right]
$$

This new obtained $\vec{u}^{* *}$ is used as the determined velocity for the subsequent calculation.

\subsubsection{Space discretization}

A high-order compact finite difference scheme is applied for space discretization based on a uniformly spaced mesh where the nodes are indexed by $i$. Compared to standard finite difference method, high-order compact finite difference scheme can reach more accurate results without coupling too many neighboring grid for differential purpose. This compact finite difference scheme achieves this superiority by mimicking the treatment of spectral method. The distribution of nodes 
$x_{i}$ are considered, with $x_{i}=(\mathrm{n}-1) \Delta x$ for $1 \leq i \leq n$. The function values at all nodes, $f_{i}=f\left(x_{i}\right)$ are given. The approximation of values of first derivative $f_{i}^{\prime}=f^{\prime}\left(x_{i}\right)$ at the node $i$ can be derived from finite difference scheme. For instance, for second- and fourth- order central difference scheme, the approximation of $f_{i}^{\prime}$, depends on information of $\left(f_{i+1}, f_{i-1}\right)$ and $\left(f_{i+2}, f_{i+1}, f_{i-1}, f_{i-2}\right)$, respectively. A general formulation for the approximation of first derivative can be written as follows [82]:

$$
\begin{aligned}
& \beta f_{i+2}^{\prime}+\alpha f_{i+1}^{\prime}+f_{i}^{\prime}+\alpha f_{i-1}^{\prime}+\beta f_{i-2}^{\prime} \\
& =c \frac{f_{i+3}-f_{i-3}}{6 \Delta x}+b \frac{f_{i+2}-f_{i-2}}{4 \Delta x}+a \frac{f_{i+1}-f_{i-1}}{2 \Delta x}
\end{aligned}
$$

where $f$ and $f^{\prime}$ are the variable and its first derivative, respectively. $\Delta x$ is the grid spacing. The relations between the coefficients $a, b, c$ and $\alpha, \beta$ can be obtained after matching the coefficients by Taylor series of relative orders, and the first unmatched coefficients determines the truncation errors of the approximation. In order to reach the sixth-order accuracy, $a=\frac{9}{14}, b=\frac{1}{9}, c=0, \alpha=\frac{1}{3}, \beta=0$ are selected [70] in the present study. Using the same strategy, the approximation of second-order derivative values $f_{i}^{\prime \prime}=f^{\prime \prime}\left(x_{i}\right)$ can be expressed as:

$$
\begin{aligned}
& \beta f_{i+2}^{\prime \prime}+\alpha f_{i+1}^{\prime \prime}+f_{i}^{\prime \prime}+\alpha f_{i-1}^{\prime \prime}+\beta f_{i-2}^{\prime \prime} \\
& =c \frac{f_{i+3}-2 f_{i}+f_{i-3}}{9(\Delta x)^{2}}+b \frac{f_{i+2}-2 f_{i}+f_{i-2}}{4(\Delta x)^{2}}+a \frac{f_{i+1}-2 f_{i}+f_{i-1}}{(\Delta x)^{2}}
\end{aligned}
$$

Similarly, the six-order compact scheme is defined as: 


$$
\begin{aligned}
& \frac{2}{11} f_{i+1}^{\prime \prime}+f_{i}^{\prime \prime}+\frac{2}{11} f_{i-1}^{\prime \prime} \\
& =\frac{3}{11}\left(\frac{f_{i+2}-2 f_{i}+f_{i-2}}{4(\Delta x)^{2}}\right)+\frac{12}{11}\left(\frac{f_{i+1}-2 f_{i}+f_{i-1}}{(\Delta x)^{2}}\right)
\end{aligned}
$$

with the coefficients $a=\frac{12}{11}, b=\frac{3}{11}, c=0, \alpha=\frac{2}{11}, \beta=0$. This approximation of sixth-order accurate scheme has the same favorable properties as the first derivative in terms of "spectral-like" resolution [82].

In Eq. (5.7), the partial differentiation with respect of velocity $u$ in convective and viscous terms are calculated by the high-order compact schemes, which are performed in Eqs. (5.20)-(5.22) for first-, and second- order derivatives, respectively. Meanwhile, different kinds of boundary conditions, such as Dirichlet, periodic, symmetrical and antisymmetric boundary conditions, are all suitable to be applied to this compact scheme. However, the treatment of boundary nodes varies under different boundary condition circumstances. More precisely, for Dirichlet boundary condition, one sided scheme should be applied on the boundary because the nodes need to be fictitiously distributed beyond the boundary, at which $\mathrm{i}=1$ or $\mathrm{n}$. Therefore, $3^{\text {rd }}$ order one-sided compact scheme [82] for both first and second derivatives are employed when $i=1$, which is expressed as:

$$
\begin{gathered}
f_{1}^{\prime}+2 f_{2}^{\prime}=\frac{-5 f_{1}+4 f_{2}+f_{3}}{2 \Delta x} \\
f_{1}^{\prime \prime}+11 f_{2}^{\prime \prime}=\frac{13 f_{1}-27 f_{2}+15 f_{3}-f_{4}}{(\Delta x)^{2}}
\end{gathered}
$$

Similarly, first and second derivatives at its adjacent point $(i=2)$ are presented as: 


$$
\begin{gathered}
\frac{1}{4} f_{1}^{\prime}+f_{2}^{\prime}+\frac{1}{4} f_{3}^{\prime}=\frac{-3 f_{1}+3 f_{3}}{4 \Delta x} \\
\frac{1}{10} f_{1}^{\prime \prime}+f_{2}^{\prime \prime}+\frac{1}{10} f_{3}^{\prime \prime}=\frac{6 f_{1}-12 f_{2}+6 f_{3}}{5(\Delta x)^{2}}
\end{gathered}
$$

These two schemes are considered as fourth-order accurate [82]. Similar formulations are implemented for the right boundary at $\mathrm{i}=\mathrm{n}$ and $\mathrm{i}=\mathrm{n}-1$.

For periodic boundary conditions, Eqs. (5.20)-(5.22) are entirely allowed to be applied, with appropriate modifications. The potential "ghost" values, such as $f_{0}, f_{-1}, f_{n+1}, f_{n+2}$, can be substituted by their counterparts $f_{1}, f_{2}, f_{n-1}, f_{n}$ in a manner as:

$$
f_{0}=f_{n}, f_{-1}=f_{n-1}, f_{n+1}=f_{1}, f_{n+2}=f_{2}
$$

For simplicity, only relations on the original values are presented here, their first and second derivatives are all applicable to Eq. (5.27).

For symmetric boundary conditions, similarly, "ghost" value can be substituted as:

$$
f_{0}=f_{2}, f_{-1}=f_{3}, f_{n+1}=f_{n-1}, f_{n+2}=f_{n-2}
$$

So do their first and second derivatives, respectively.

\subsubsection{Boundary condition implementation}

As depicted in Fig. 5.1, for the sake of description convenience, (1) and (2) stand for inlet and outlet surfaces; (3) and (4) represent the top and bottom surfaces, while (5) and (6) denote the front and rear side of transverse surfaces in y-direction, respectively.

In present study, when height of immersed obstacle is equal to domain height (Sections 5.4.1 126 
5.4.4), which means the object penetrates the whole domain in z-direction, periodic boundary condition is applied in (3) and (4) boundaries. Symmetric boundary conditions $\left(\frac{d u}{d y}=0\right)$ are given to transverse boundaries (5) and (6), which can be interpreted as free slip boundary condition, as zero normal velocity is given. At the inlet surface (1)), the boundary conditions are set as:

$$
\left\{\begin{array}{c}
u=U_{\infty} \\
v=w=0 \\
\frac{d p}{d x}=0
\end{array}\right.
$$

and at the outlet (2), the boundary conditions are

$$
\left\{\begin{array}{c}
p=0 \\
\frac{d u}{d x}=\frac{d v}{d x}=\frac{d w}{d x}=0
\end{array}\right.
$$

No-slip boundary condition $\left(u=u_{\text {wall }}=0\right)$ is employed for all surfaces of solid immersed obstacle while all other surfaces remain the same.

While when the height of immersed obstacle is finite (shorter than the domain height, Section 5.4.5), Dirichlet boundary conditions are applied on all surfaces. Blasius solution [88] for flat plate flow is added to two transverse surfaces ((5) and (6), top surface (3), as well as inlet surface (1), so that the thickness of boundary layer is properly considered. The continuous of velocity on all directions can be guaranteed after utilizing this manner. Besides, no-slip boundary condition ( $u=u_{\text {wall }}=0$ ) is employed for all surfaces of solid immersed obstacle and bottom surface (4)). 


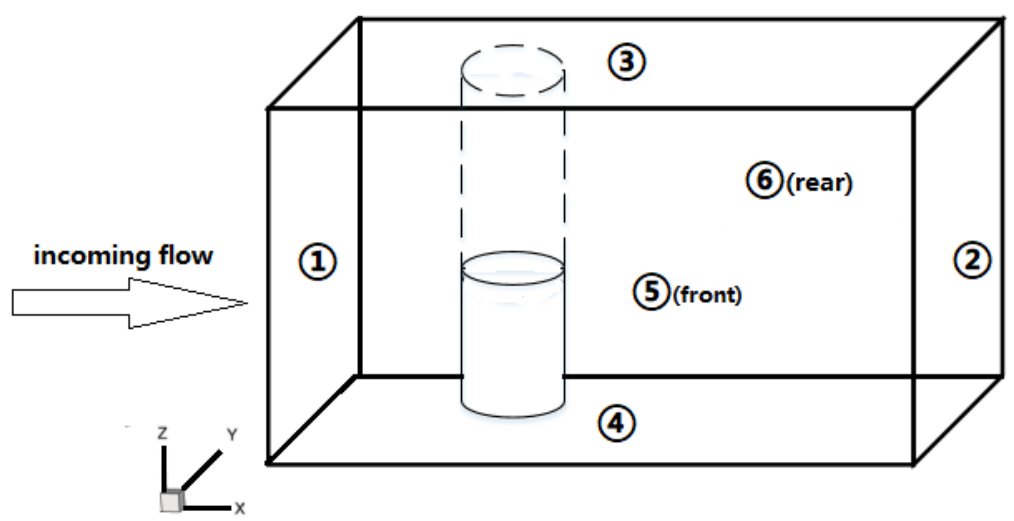

Fig. 5.1 Schematic of domain and boundary condition interpretation

\subsubsection{IB-FDM framework}

A typical procedure for each time step of the framework is described as follows:

1. Initialize the computational domain;

2. Given the immersed-boundary location at time step $n+1$, identify the fluid, solid and solid boundary points on the Eulerian grid;

3. Obtain forcing term via feedback forcing model;

3.a Obtain the velocity $\vec{U}_{B}$ at Lagrangian points from Eqs. (5.9),(5.10) and (5.13);

3.b Obtain the restoring force $\vec{F}_{R S T}$ at Lagrangian points via Eq. (5.16);

3.c Distribute the restoring force exerted on Lagrangian points to Eulerian points by Eq. (5.12);

4. Compute the velocity field $\vec{u}^{*}$ by solving Eq. (5.3);

5. Compute intermediate velocity $\vec{u}^{* *}$ by solving Eq. (5.4);

6. Compute the pressure $\nabla \tilde{p}^{n+1}$ by solving pressure Poisson equation Eq. (5.5); 
7. Correct divergence-free fluid velocity $\vec{u}^{n+1}$ at $\mathrm{n}+1$ time step by solving Eq. (5.6);

8. Go back to Step 3 if time-marching is still needed; otherwise, stop the computation.

Note that steps 4 and 5 will be replaced by solving Eq. (5.19) directly when the enhanced prediction-correction algorithm is applied.

\subsection{Validation}

To verify the validity and accuracy of the proposed feedback forcing based Incompact3D framework, a series of incompressible viscous benchmark cases, including flows past stationary/moving cylinders, flow over oscillating cylinder and flow over finite height square prism, are chosen as numerical experiments. These problems have been studied extensively, and some existing results reported elsewhere will serve here as references for comparison use.

In all present cases, unless otherwise specified, the computational domain is fixed as a cuboid with the domain size of $21 \times 16 \times 1$ in the $x-, y-$, and $z-$ direction respectively. A uniform mesh with the size of $379 \times 289 \times 32$ is taken. The diameter of cylinder is 1 , with a height of 1 . The fluid density is set at $\rho=1.0$ and the free incoming stream velocity is $U_{\infty}=1.0$. The mesh resolution is kept the same as default settings of published Incompact3d version, which is used to solve flow around a cylinder configuration at $\mathrm{Re}=300$ [69]-[70]. Considering the Reynolds number of all cases discussed in present work is less than 300 , the mesh resolution should be conservative and reliable.

Different from the definition of drag and lift coefficients (Eq. (2.37) and (2.39)) in 2D scenario, 
the drag coefficient $C_{d}$ or $C_{x}$ is then defined as:

$$
C_{d}=\frac{2 F_{D}}{\rho U_{\infty}^{2} S_{x}}
$$

and the lift coefficient $C_{l}$ or $C_{y}$ is defined as:

$$
C_{l}=\frac{2 F_{L}}{\rho U_{\infty}^{2} S_{y}}
$$

$S_{x}, S_{y}$ denotes the projection area in $x$ - and $y$-direction, respectively. Similarly, the force coefficient in spanwise direction (z-direction) is determined as:

$$
C_{z}=\frac{2 F_{Z}}{\rho U_{\infty}^{2} S_{z}}
$$

where $F_{Z}$ and $S_{z}$ are the force and projection area in z-direction, respectively.

\subsubsection{Flow over stationary cylinder at $\operatorname{Re}=40$}

The incompressible viscous flow over a stationary cylinder at $\mathrm{Re}=40$ is a classic benchmark case, which has been studied extensively. The cylinder is placed throughout the whole z-direction. Under this circumstance, the flow features in each cross-section along z-direction should be equivalent to a traditional two-dimension flow over a cylinder case after the flow reaches steady state. Thus, periodic boundary condition is employed at the top and bottom surfaces in order to maintain the characteristics in this case. This flow features symmetric streamlines about the central horizontal line passing through the center of cylinder from two dimension scenario, which is confirmed again by the present 3D simulations results as shown in Fig. 5.2. In particular, Fig. 5.2 
also clearly demonstrates a pair of symmetric recirculating eddies behind the cylinder, while there is no indication of flow penetration across the boundary of the immersed object, confirming the success of incorporation of the feedback forcing based IB approach in the Incompact3D framework. Another important remark can be made on the satisfaction of the no-slip boundary condition on the surface of the cylinder in the present model, since Fig. 5.3 illustrates the velocity strictly remains zero at the solid boundary.

Table 5.1 groups the drag coefficient and recirculation zone length data obtained using this proposed framework and those reported by other literature, indicating the present study can produce reliable numerical results when dealing a traditional incompressible flow associated with an immersed obstacle scenario since they satisfactorily match with other reported data.

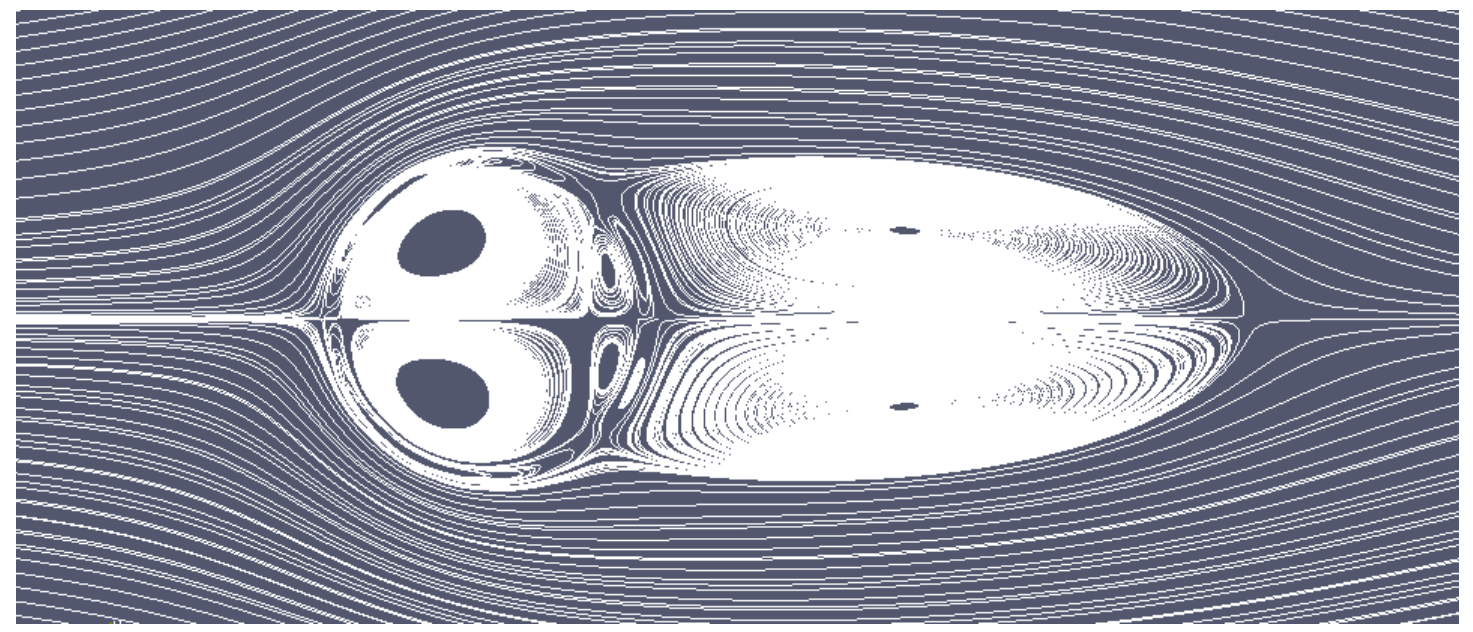

Fig. 5.2 Distributions of streamline, flow over a stationary cylinder at $\operatorname{Re}=40$ 


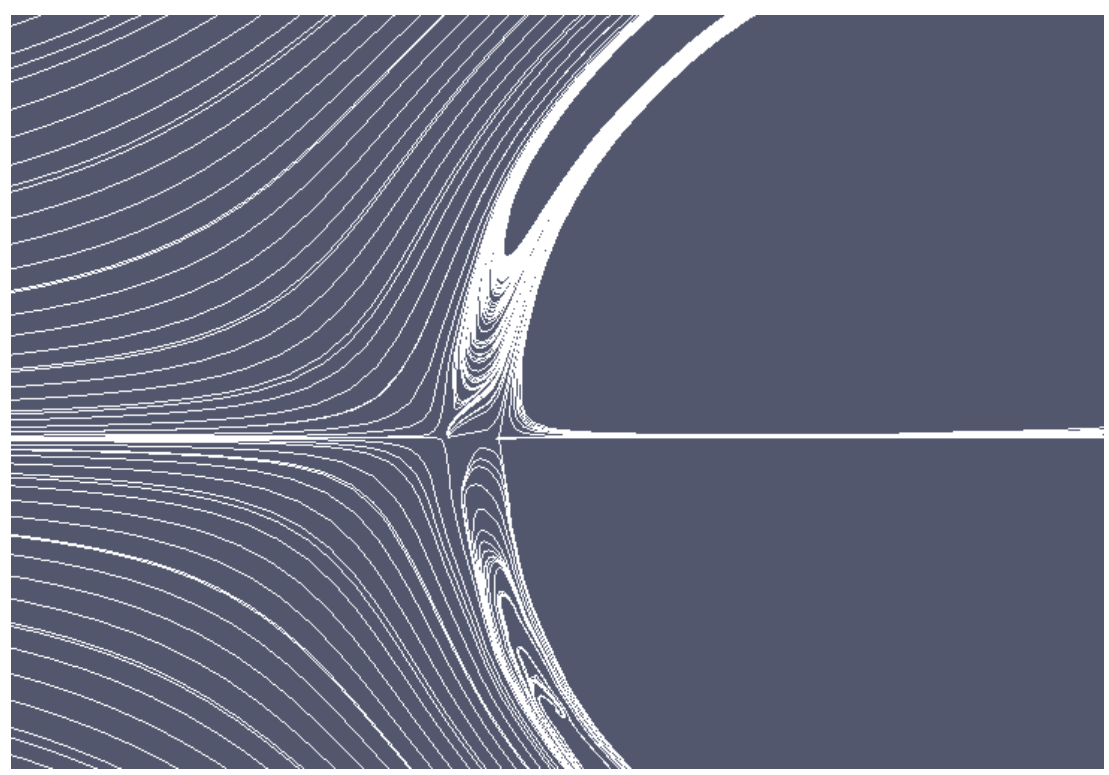

Fig. 5.3 Zoom-in of streamline at the vicinity of cylinder

Table 5.1 Comparison of drag coefficient and length of recirculation zone at $\mathrm{Re}=40$

\begin{tabular}{lll}
\hline References & Drag coefficient $C_{d}$ & Length of recirculation zone L \\
\hline Fornberg [89] & 1.498 & 2.25 \\
Shukla et al. [90] & 1.550 & 2.34 \\
Niu et al. [91] & 1.589 & 2.26 \\
Wu et al. [55] & 1.554 & 2.30 \\
\hline Present & 1.518 & 2.29 \\
\hline
\end{tabular}

\subsubsection{Flow over moving cylinder at $\mathrm{Re}=\mathbf{4 0}$}

Furthermore, flow over a moving cylinder case will be tested, which is aimed to validate its 
capability in moving boundary problems, in particular for translational motion. Here, the free stream velocity keeps $U_{\infty}=0.95$ while the cylinder itself also moves towards the incoming flow constantly at $U_{\text {object }}=-0.05$, so the incoming flow velocity relative to the moving cylinder remains to be 1 . This modification generates a horizontal translating velocity for the cylinder, which creates a scenario of moving boundary. However, compared to the first test case with a stationary cylinder discussed in Section 5.4.1, the modification made here is not expected to alter the flow patterns, since the incoming flow velocity relative to the moving cylinder evidently remains the same, so that the characteristic velocity defined is unchanged.

Unsurprisingly, in this moving boundary case, despite the translation of the cylinder, the flow behavior around the cylinder looks identical and steady if observed from the cross-section in x-direction, focusing on moving cylinder itself. Fig. 5.4 depicts the streamlines obtained in the moving scenario, and exhibits no difference from that in the stationary case as illustrated in Fig. 5.2. From three dimensional perspective, as the contour of velocity magnitude shown in Fig. 5.5, velocity keeps the same magnitude along z-direction, and the contour in each cross-section is also depicted in Fig. 5.6. These two figures again confirm the flow patterns should be equivalent to traditional two-dimensional scenario in each plate. The root mean square of velocity in z-direction $(w)$ is 0.00073 , which proves the three-dimension effect in this case is very weak, and the aforementioned results are accurate and reliable. Additionally, this test reveals the capability of the feedback forcing based IB approach for simulation of flow problems with obstacle moving in the 133 
fluid.

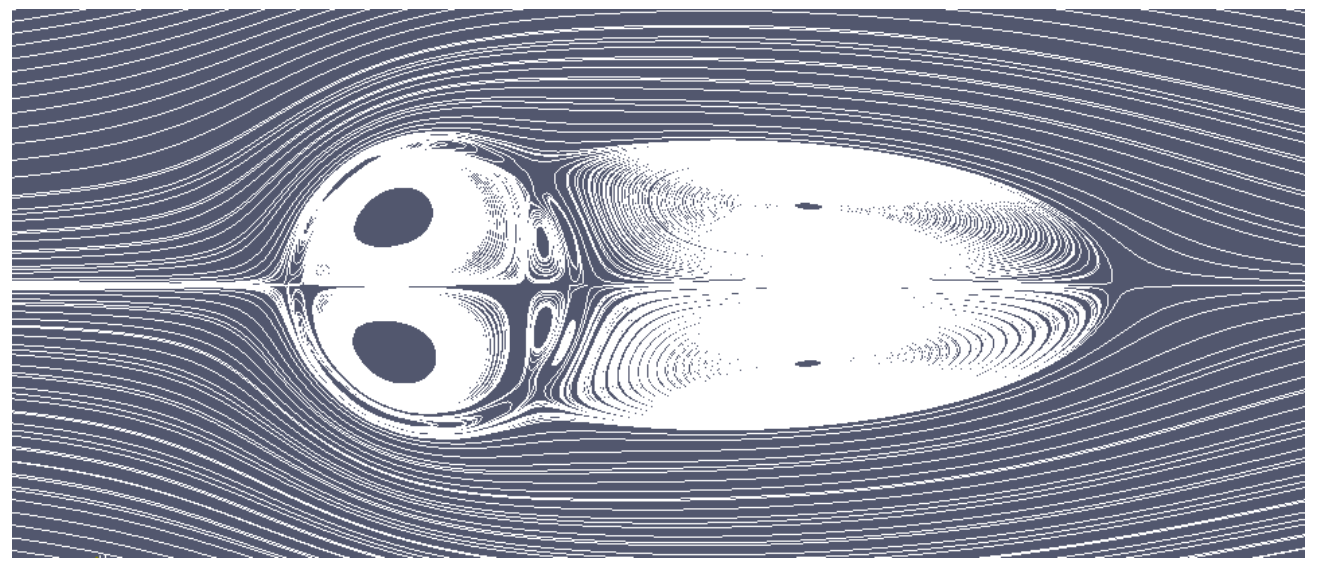

Fig. 5.4 Distributions of streamline, flow over a moving cylinder at $\operatorname{Re}=40$

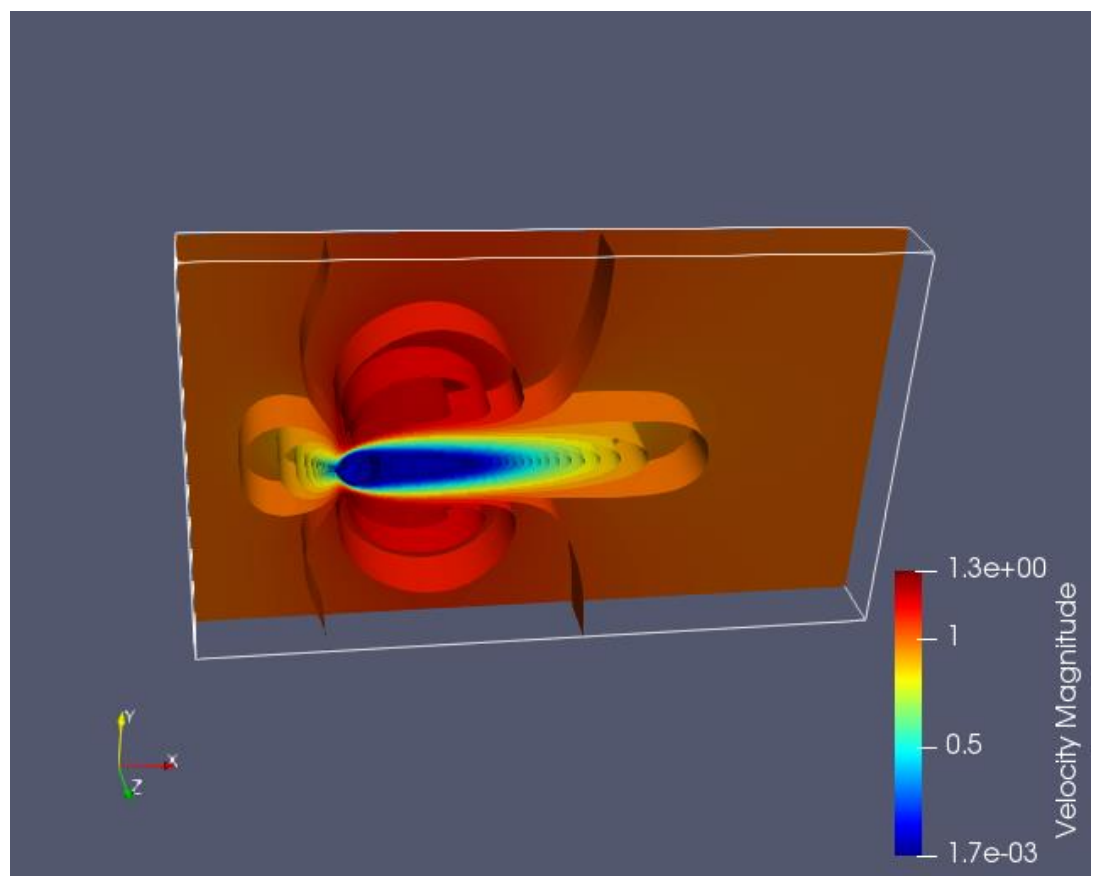

Fig. 5.5 Contour of velocity magnitude in three dimension at $\operatorname{Re}=40$ 


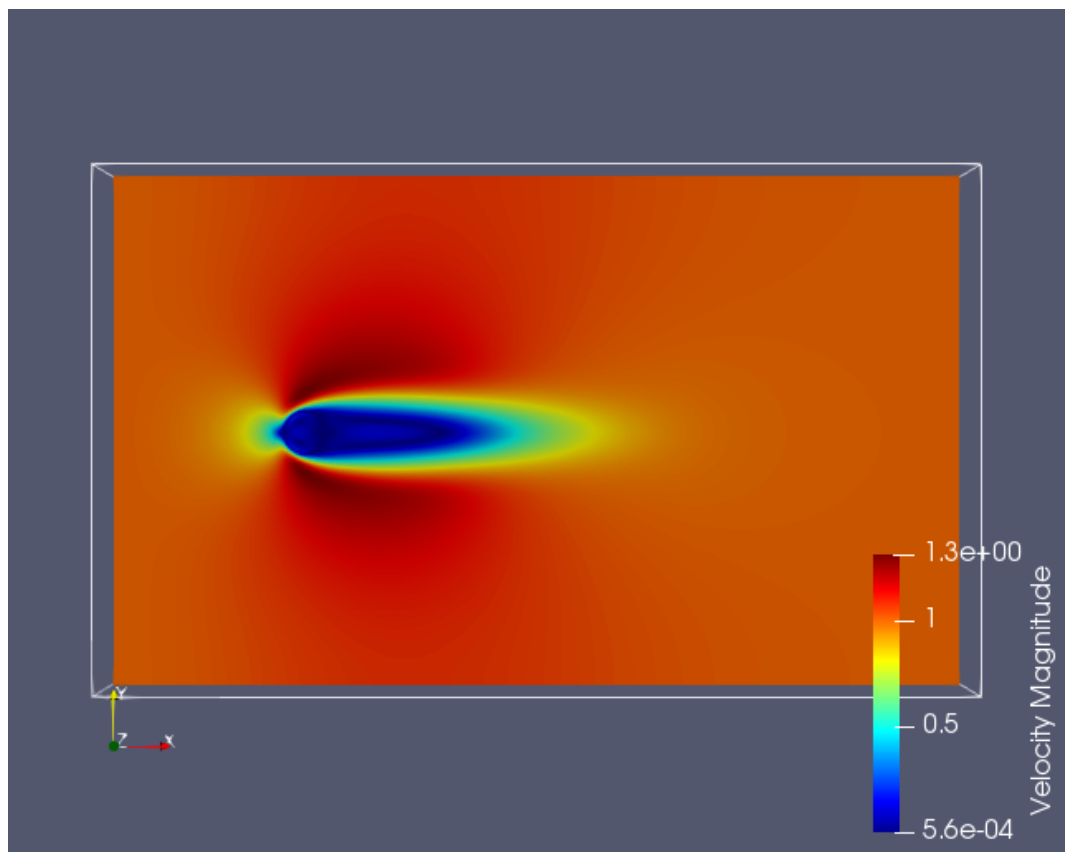

Fig. 5.6 Contour of velocity magnitude in one cross-section $(z=0.5)$ at $\operatorname{Re}=40$

\subsubsection{Flow over moving cylinder at $\operatorname{Re}=100$}

For simulating the unsteady flow at an elevated Reynolds number $\operatorname{Re}=100$, the free incoming stream moves at $U_{\infty}=0.95$ again, while the cylinder itself is allowed to be horizontally migrating a constant velocity $U_{\text {object }}=-0.05$ in the opposite direction to the free stream, and all other conditions remain unchanged. Fig. 5.7 shows the contour of velocity magnitude in three-dimensional view at one time instant, and Fig. 5.8 - Fig. 5.9 illustrate the evolution of the streamlines and vorticity contours in the vicinity of the cylinder at two different time steps, respectively. Vortex shedding phenomenon can be easily observed in Fig. 5.7, and the velocity magnitude contours do not change in different cross-sections along z-direction. Fig. 5.9 contains two 
different instantaneous view of the vorticity contours, illustrating both the motion of the cylinder itself and the evolution of the Karman vortex street. As seen from the vorticity contours in Fig. 5.9, the vortex is shedding at a constant frequency. Here, the Strouhal number (Eq.(2.36)) is employed to examine the dimensionless frequency when the vortices are shed from the body:

This vortex shedding frequency $f_{q}$ can be acquired by a look into the time evolution of the drag and lift coefficients depicted in Fig. 5.10. The oscillation of the two coefficients looks periodic, which demonstrates the flow field varies periodically when the vortices are shed from the cylinder. Table 5.2 compares the time-averaged drag coefficient, $\bar{C}_{d}$, and Strouhal number, $S t$, of this unsteady flow case obtained using the present model to those obtained elsewhere, which shows that the present results are within the range of values reported by the references.

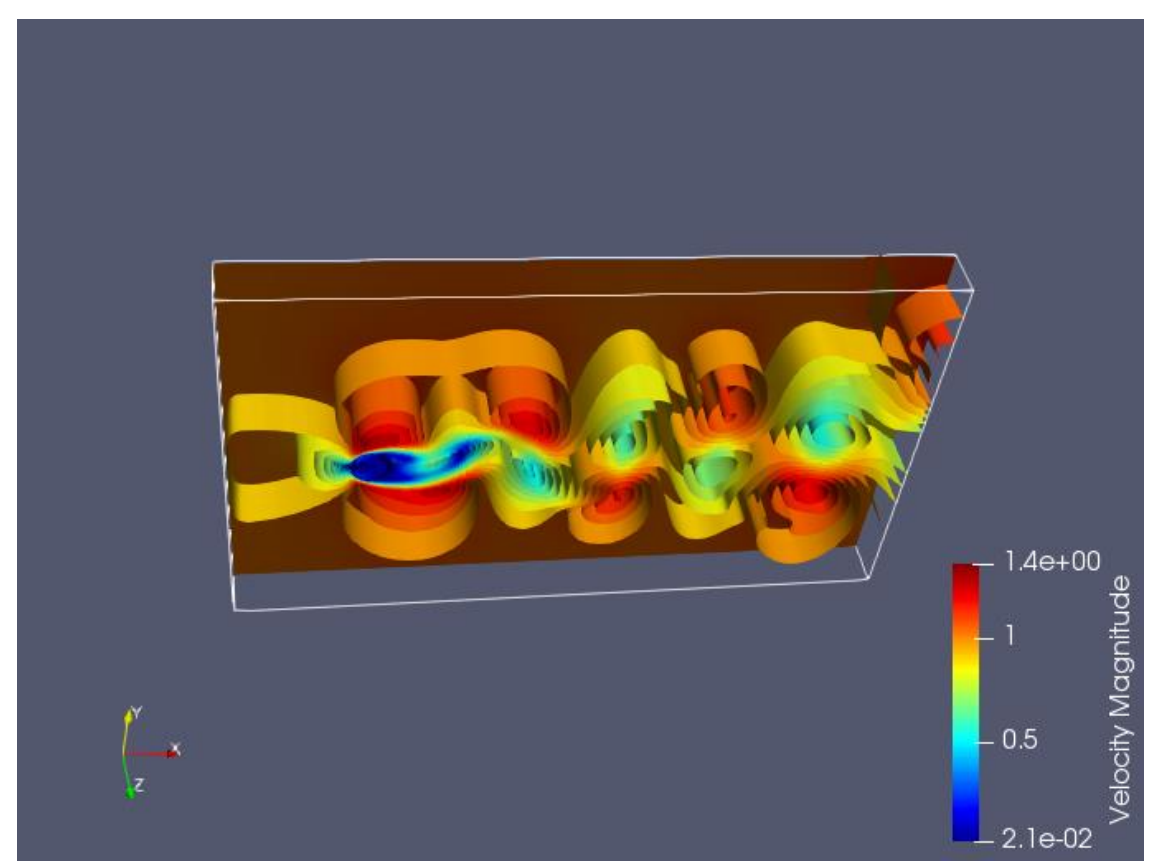

Fig. 5.7 Contour of velocity magnitude in three dimension at $\mathrm{Re}=100$ 


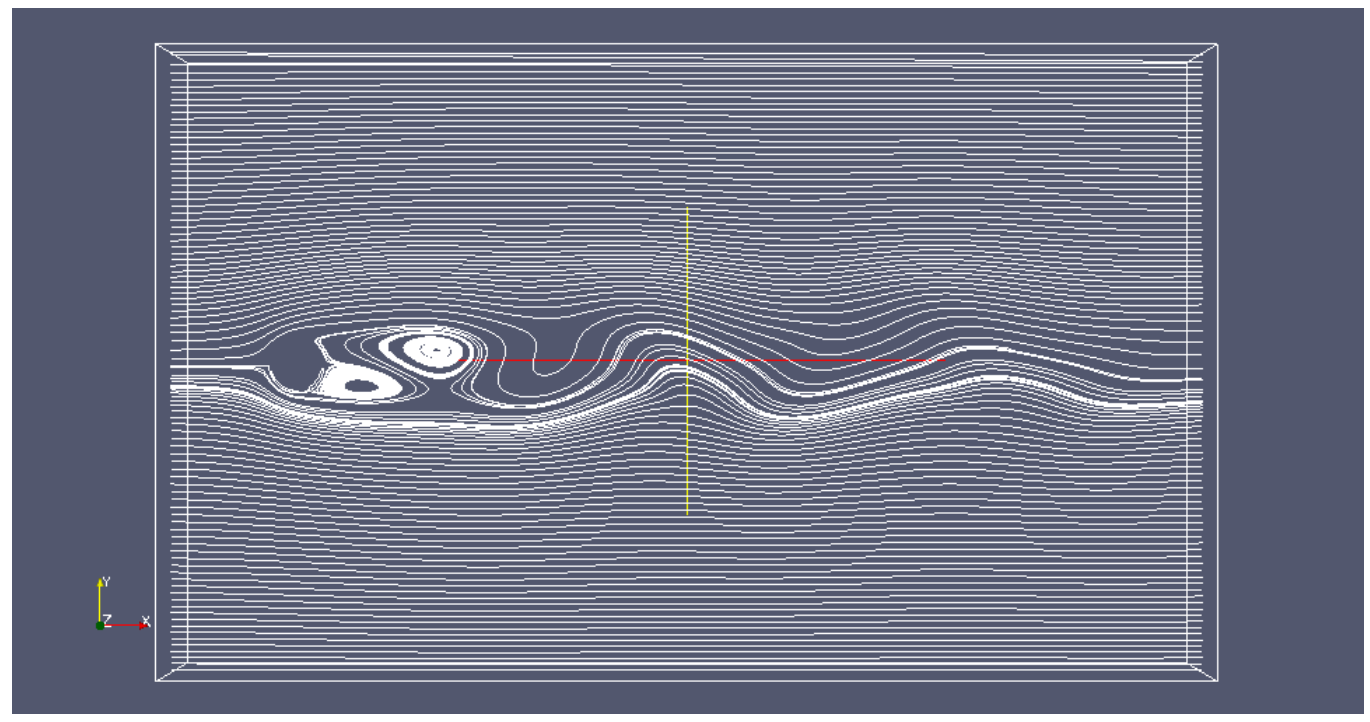

(T1)

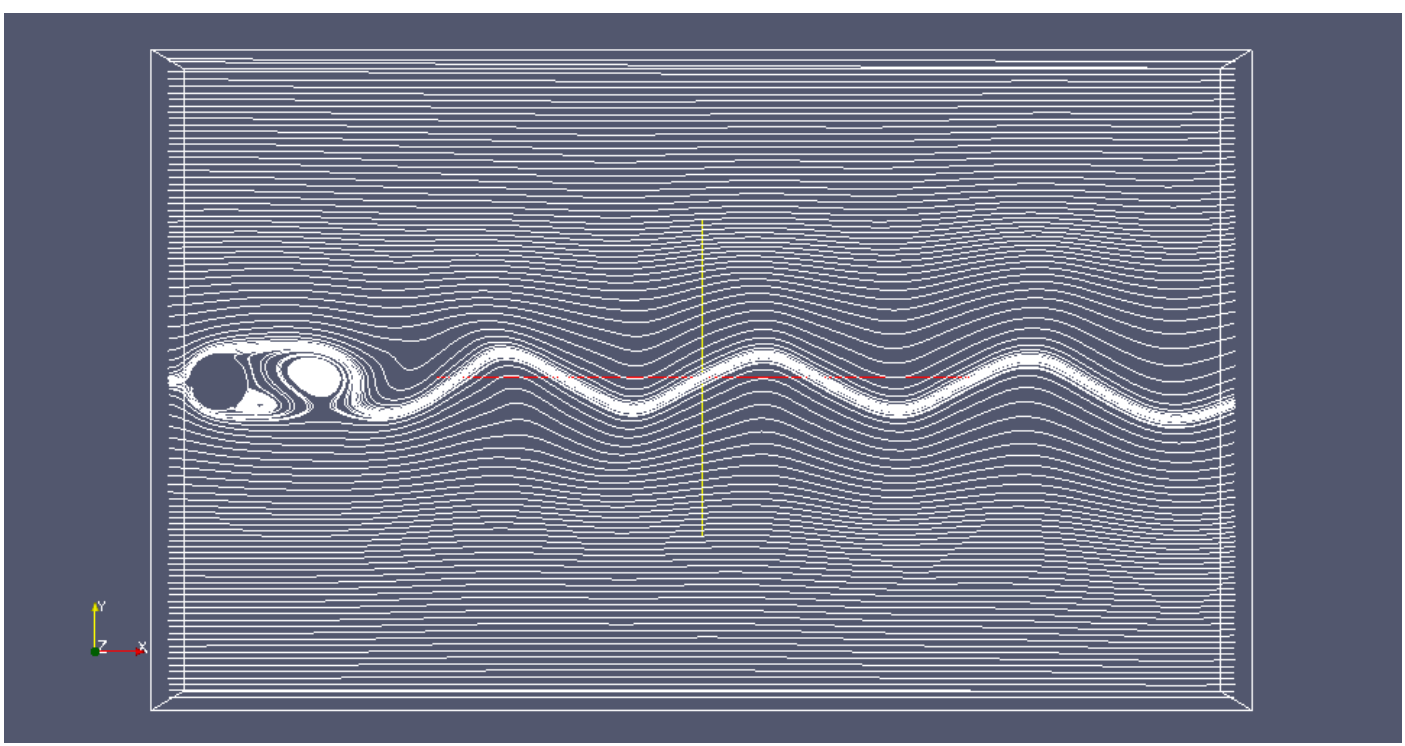

(T2)

Fig. 5.8 Streamlines for flow over moving cylinder at $\mathrm{Re}=100$ (Two different time instants, $\mathrm{T} 1$ and T2) 

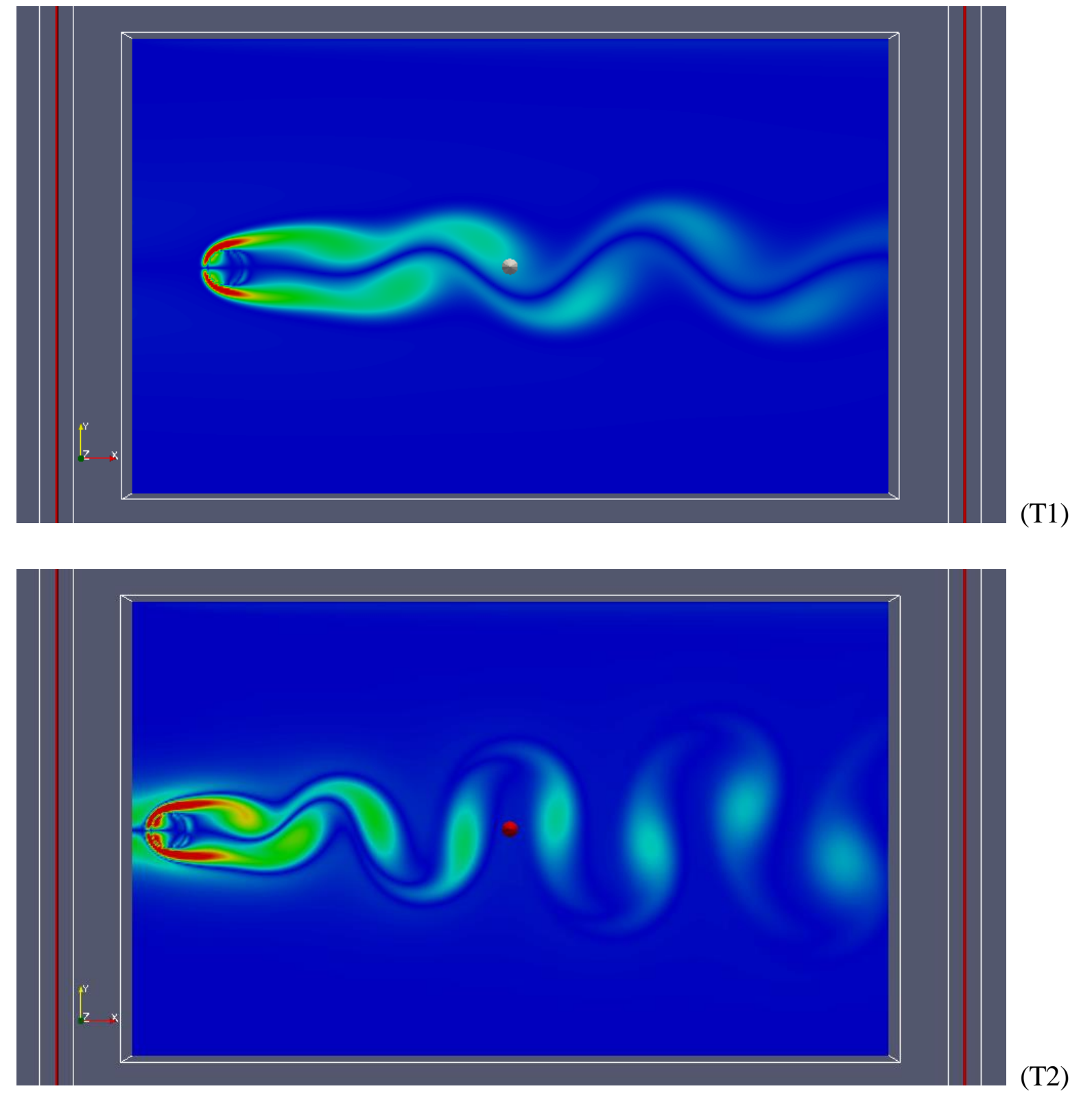

Fig. 5.9 Vorticity contours for flow over moving cylinder at $\mathrm{Re}=100$

(Two different time instants, T1 and T2) 
Table 5.2 Comparisons of time-averaged drag coefficient and Strouhal number at $\operatorname{Re}=100$

\begin{tabular}{|c|c|c|}
\hline References & Drag Coefficient $\bar{C}_{d}$ & Strouhal number \\
\hline Wu et al. [55] & 1.364 & 0.163 \\
\hline Saiki et al. [92] & 1.26 & 0.171 \\
\hline Silva et al. [93] & 1.39 & 0.161 \\
\hline Russell et al. [94] & 1.43 & 0.175 \\
\hline Present & 1.301 & 0.167 \\
\hline
\end{tabular}

Considering the extended iteration steps to capture the periodic oscillation (as shown in Fig. 5.10), a prediction-correction algorithm will be embedded in this case. As described in Sec. 5.3.5, Eq. (5.19) is applied instead to calculate $\vec{u}^{* * *}$, while all other parameters and solving processes remain the same. Comparing with previous regular fraction step solver, the converging time can be reduced significantly, which is depicted in Fig. 5.10. Evolutions of both drag and lift coefficients with respect to time iterations are illustrated. For original feedback forcing based method, periodic variation occurs after around 380,000 iterations, which means the Karman vortex shedding phenomenon abovementioned can only be captured after that time instant. However, when prediction-correction scheme is added, the evolution can be regarded to converge at around 220,000 iterations, which is distinctively less than original test. After that time instant, same periodic patterns 
can be observed. Despite the difference in convergent steps, the drag and lift coefficients for each trial are almost the same. Thus, prediction-correction scheme is proved to be an efficient method in accelerating the converge procedure in IB method, and in this case, the converging steps can be shortened up to 42 percent after applying prediction-correction scheme.

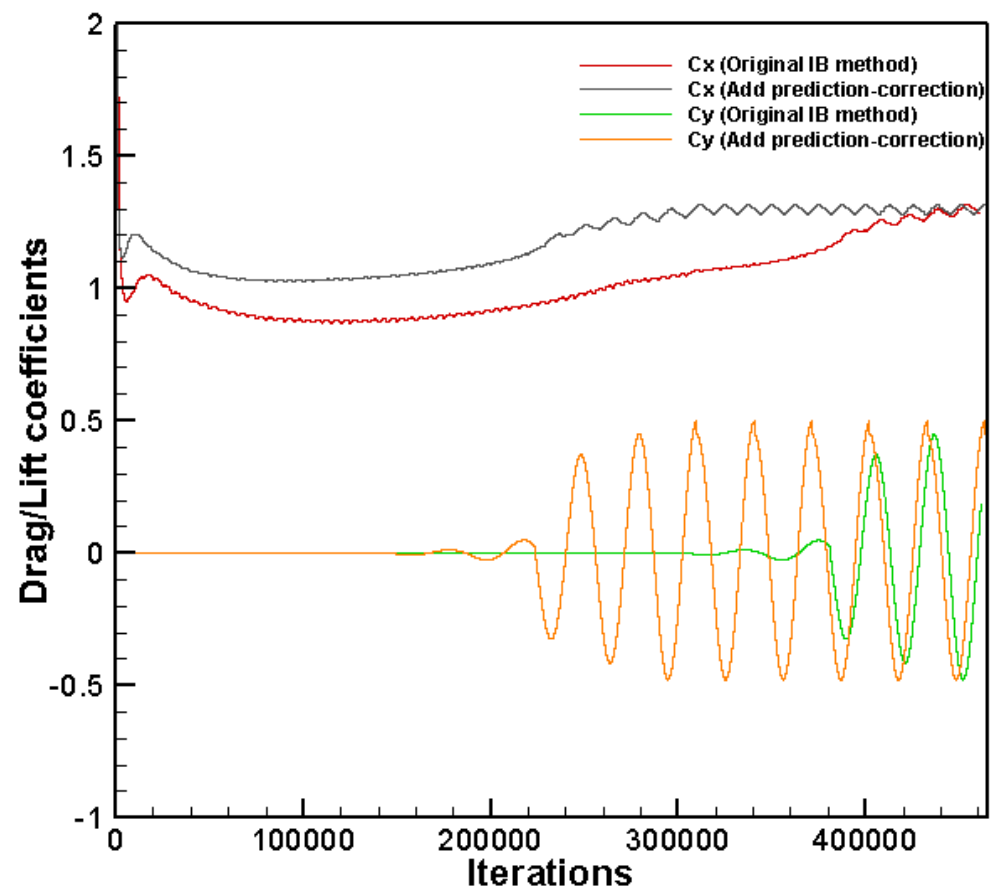

Fig. 5.10 Comparison between original and prediction-correction scheme added results

Besides, the numerical accuracy with respect to time is tested as well. Both original and prediction-correction embedded framework will be measured and compared respectively. According to the evaluation method proposed by Roache [95], suppose $\phi$ is the exact solution; in presence of a numerical solution, $\tilde{\phi}$, which is obtained by using a time step with $\xi$ as the index of time step, the dependency of exact solution, $\phi$, on relevant factors, including the time step index, $\xi$, can be 
expressed as follows [95]:

$$
\phi=\tilde{\phi}+\chi \xi^{\gamma}
$$

where both $\chi$ and $\gamma$ are constants, and particularly, $\gamma$ represents the accuracy order of the numerical method with respect to time. Therefore, three different time steps are tested, for both original IB method, and prediction-correction scheme method. Three sets of results, which are Test A, B and C standing for three different time steps ( $d t=0.0001,0.00012,0.00015)$, are required to build a well-posed algebraic system for the solutions of three unknowns $\phi, \chi$ and $\gamma$. Here, drag coefficient $C_{d}$ is served as the solution under investigation. The results for both methods are listed at Table 5.3. Drag coefficients for both original and prediction-correction schemes in different time step are very close, and after solving these two equation systems according to Eq. (5.34) separately $\gamma_{1}=2.26$, and $\gamma_{2}=1.97$ are obtained. This indicates that, after embedding the prediction-correction scheme into IBM, the numerical order of accuracy with respect to time is increased to 2.26 from its original order of accuracy 1.97, with an increase of $14.7 \%$. Therefore, the original method can be viewed as approximately second-order accuracy, while after adding prediction-correction scheme, this framework owns more than second-order accuracy, and proved to be more precise with respect to time. 
Table 5.3 Tests for determining the numerical accuracy

\begin{tabular}{|c|c|c|c|}
\hline Test case & Time step $(\xi)$ & $\begin{array}{l}\text { Numerical drag } \\
\text { coefficient }\left(\tilde{\phi}_{1}\right)\end{array}$ & $\begin{array}{l}\text { Numerical drag } \\
\text { coefficient }\left(\tilde{\phi}_{2}\right)\end{array}$ \\
\hline A & 0.0001 & 1.3015 & 1.3008 \\
\hline B & 0.00012 & 1.2976 & 1.2961 \\
\hline $\mathrm{C}$ & 0.00015 & 1.2900 & 1.2875 \\
\hline
\end{tabular}

Note: $\tilde{\phi}_{1}$ represents result (drag coefficient) for prediction-correction scheme added method; $\tilde{\phi}_{2}$ represents result (drag coefficient) for original IB method.

\subsubsection{Oscillating cylinder case at $\mathrm{Re}=100$}

In this section, a more complex flow over a rotationally oscillating circle cylinder will be tested to verify the present framework, in particular focusing on the rotational component. The motion of rotationally oscillating cylinder is same as the work by Wu et al. [51] and Choi et al. [52], and can be prescribed by the following time-dependent angular velocity:

$$
\omega(t)=\omega_{0} \sin (2 \pi f t)=A \frac{2 u_{\infty}}{d} \sin \left(2 \pi S t_{f} \frac{u_{\infty}}{d} t\right)
$$

where $\omega_{0}$ and $f$ are the maximum rotatory amplitude and the frequency, respectively. These two parameters can be normalized by introducing two dimensionless parameters: the Strouhal number, $S t_{f}=f d / u_{\infty}$, and the normalized rotation rate, $A=0.5 \omega_{0} d / u_{\infty}$. Here, $A=2.0$ and $\operatorname{Re}=100$ 
are used in the present simulation, in order to match with the references [51] and [52].

As shown in Fig. 5.11, the instantaneous vorticity field obtained using present approach compares well with that of $\mathrm{Wu}$ et al. [51] and Choi et al. [52] when $S t_{f}=0.163$. The vortex shedding phenomenon can be observed clearly using present method, and the structure of the vortexes released from the obstacle are overall the same. The root mean square of velocity in Z-direction is as small as 0.0013 , which confirms the weakness of three-dimension effect in this case, and the flow pattern in one single cross-section is considered to be identity. From Table 5.4, when the $S t_{f}$ increases to 0.4 , both the time-averaged drag coefficient $\bar{C}_{d}$ and maximum amplitude of lift coefficient $C_{l}^{\prime}$ are found comparable with the results in Refs. [51] and [52]. This indicates that present framework can achieve reliable simulation results dealing with both rotating and translating flow components after both quantitative and qualitative validation.

Table 5.4 Comparison of drag and lift coefficients at $\mathrm{Re}=100$ for oscillating cylinder case

\begin{tabular}{lcc}
\hline References & Drag coefficient $\overline{C_{d}}$ & Lift coefficient $C_{l}^{\prime}$ \\
\hline Wu et al. [51] & 1.302 & 0.321 \\
Choi et al. [52] & 1.231 & 0.299 \\
Present & 1.301 & 0.330 \\
\hline
\end{tabular}




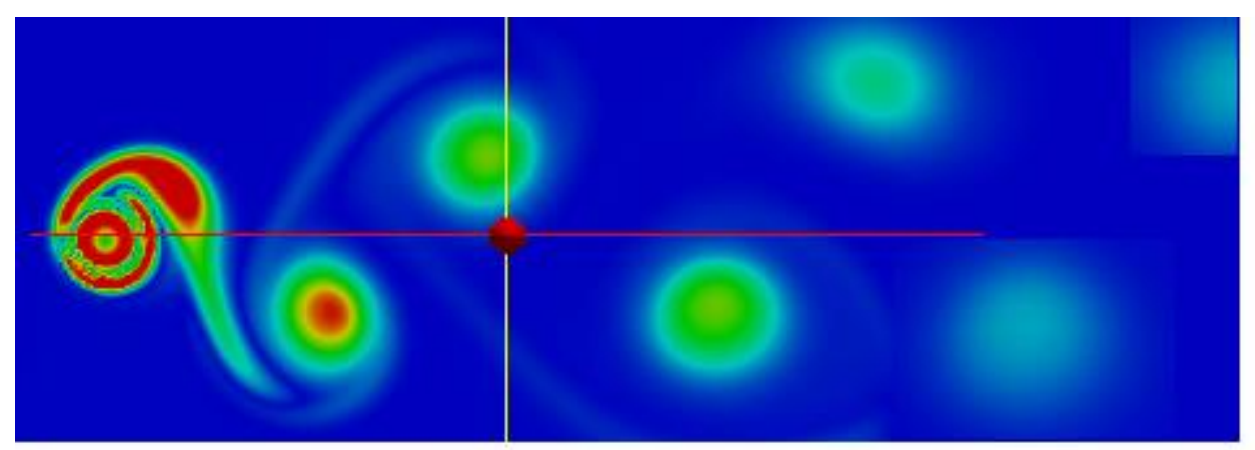

a.

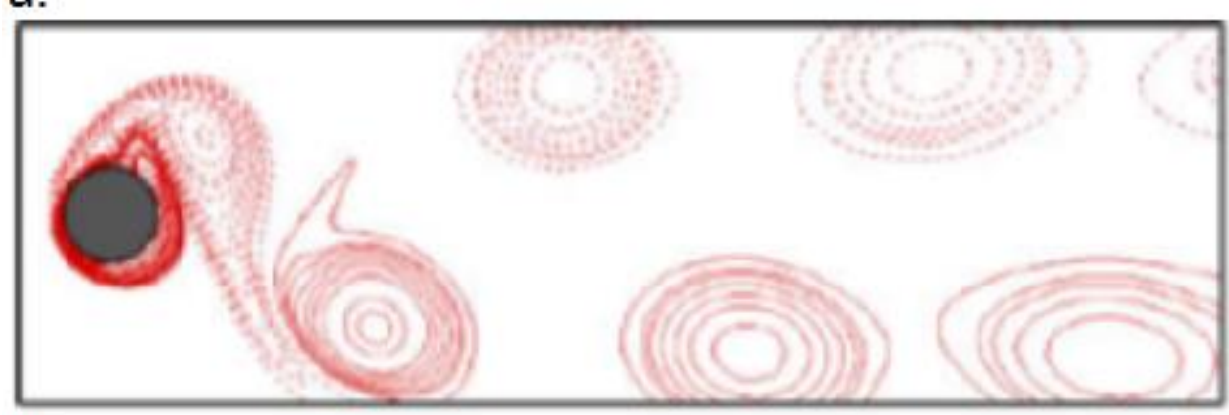

b.

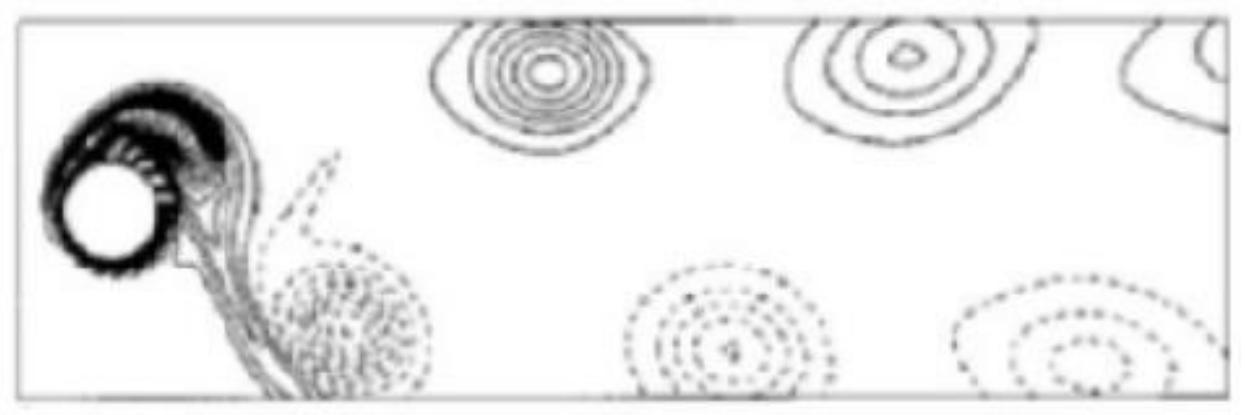

c.

Fig. 5.11 Vorticity contour results from (a) present method; (b) Wu et al. [51]; (c) Choi et al. [52].

\subsubsection{Flow around a wall-mounted square prism at $\mathrm{Re}=\mathbf{4 0}$ and 85}

Aforementioned cases for validation purpose from Sec. 5.4.1 to 5.4.4, are all conducted under a relatively 'thin' rectangular domain with the cylinder placed throughout the whole z-direction, such that flow features in each cross-section along z-direction can be considered as comparable to two 
dimension scenario. However, flow over a finite wall-mounted square prism will be introduced in this sub-section. Not only can the proposed feedback forcing based Incompact3d framework be validated again, but also a thorough three dimension framework will be built after this case, which is a prerequisite for further tornado investigation.

The geometry and computational domain designed for this case are schematically presented in Fig. 5.12. For the sake of comparing with previous reference, all dimensions are tailored in accordance with Rastan's work [96]. The height to width ratio of the prism, $h / d$, is set to 7 , and the cross-section of the prism is a square with the length fixed as $d$. To minimize the domain boundary effect on the flow, the computational domain is selected as $\mathrm{H}=13 \mathrm{~d}, \mathrm{~L}_{\text {front }}=5.5 \mathrm{~d}, \mathrm{~L}_{\text {back }}=17.5 \mathrm{~d}$, and $\mathrm{W}=16 \mathrm{~d}$. Notably, the Reynolds number here is defined as $\operatorname{Re}=\rho U_{\infty} d / \mu$.

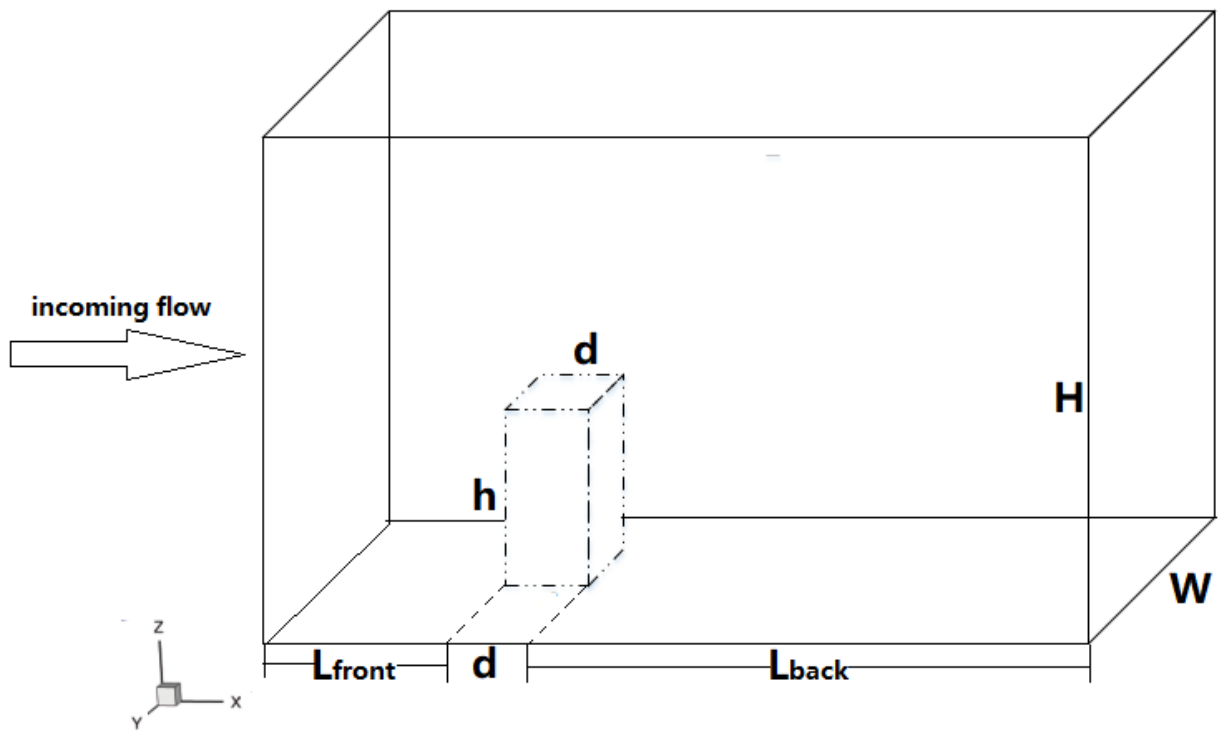

Fig. 5.12 Schematic of computational domain

An observation of Fig. 5.13 reveals the mean spanwise velocity and streamlines on the $\mathrm{X}-\mathrm{Z}$ 
plane at $y=W / 2$. Both spanwise and streamwise sizes of the recirculation can be observed, and no streamline penetration inside and outside the solid boundary, which again verifies the no-slip boundary condition is strictly guaranteed. On the other hand, both the spanwise velocity contour and streamlines are similar to those captured from [96], and force coefficients in all three directions are also compared. Table 5.5 shows that the results calculated from the proposed framework are in excellent agreement with the reference data [96].
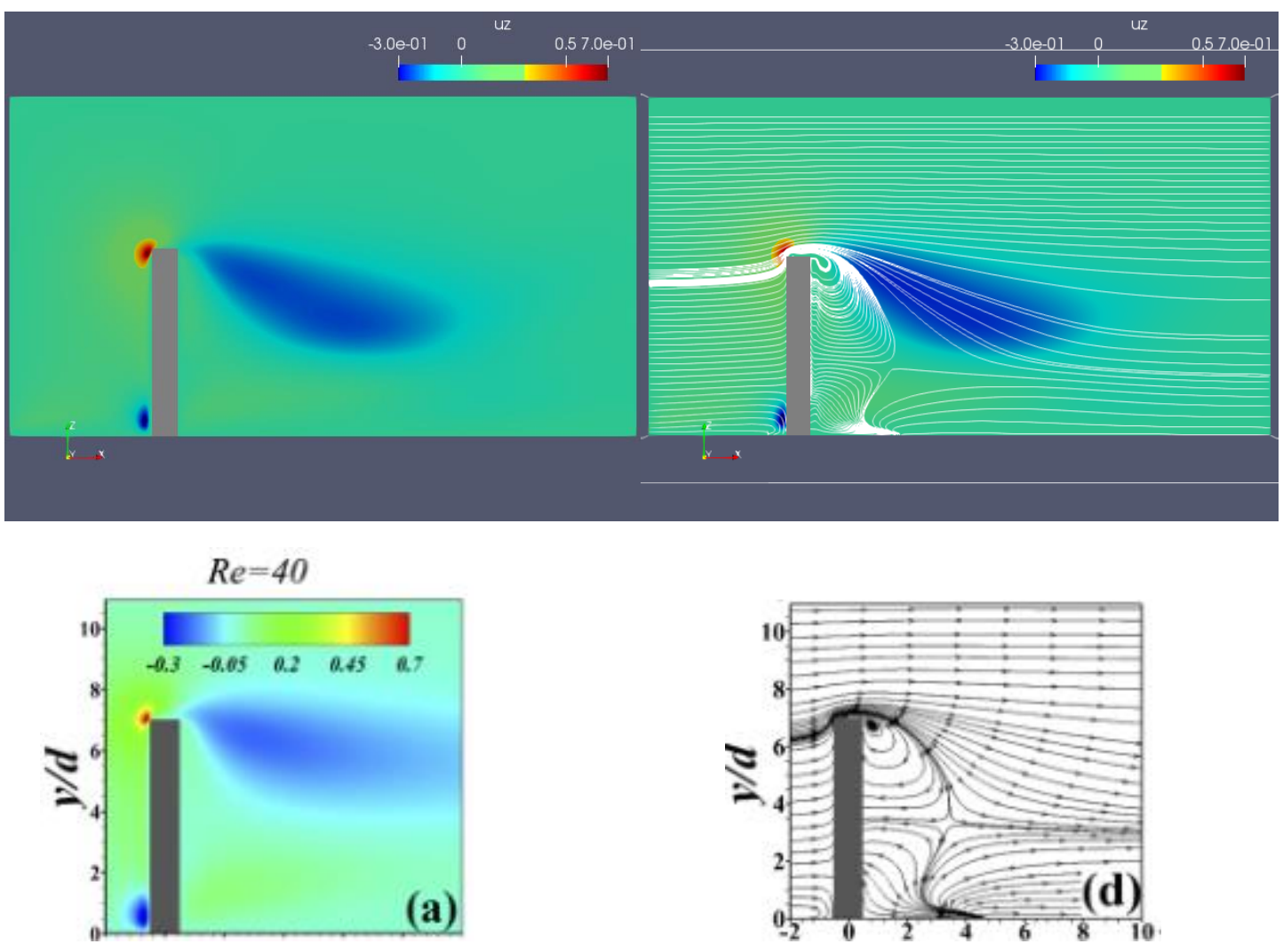

Fig. 5.13 Mean velocity in z-direction and streamlines on the mid-transverse plane $(y=W / 2)$ at $\mathrm{Re}=40$ (top: present results; bottom: captured from [96]) 
Table 5.5 Comparison of force coefficients at $\mathrm{Re}=40$ and 85 for flow over wall-mounted prism

\begin{tabular}{|c|c|c|c|c|c|c|}
\hline \multirow[t]{2}{*}{$\mathbf{R e}$} & \multicolumn{2}{|c|}{$\mathrm{Cd}_{\mathrm{d}}(\mathrm{x}$-direction) } & \multicolumn{2}{|c|}{$\mathrm{Cl}_{\mathrm{l}}(\mathrm{y}$-direction $)$} & \multicolumn{2}{|c|}{$\mathbf{C}_{\mathbf{z}}$ (z-direction) } \\
\hline & Ref.[96] & Present & Ref.[96] & Present & Ref.[96] & Present \\
\hline $\operatorname{Re}=40$ & 1.856 & 1.890 & $\rightarrow 0$ & $\rightarrow 0$ & 0.171 & 0.177 \\
\hline $\operatorname{Re}=85$ & 1.425 & 1.505 & $\rightarrow 0$ & $\rightarrow 0$ & 0.151 & 0.158 \\
\hline
\end{tabular}

With satisfactory agreement achieved in qualitative and quantitative comparison through series of validation cases discussed, the proposed feedback forcing based Incompact3d framework is proved to produce reliable and accurate numerical results dealing with three dimensional fluid-solid interaction problems. Besides, this framework incorporates the advantages of both original Incompact3d package [81] and feedback forcing based immersed boundary method on numerical accuracy, parallelization, and computing efficiency, and it is a powerful and promising tool for tornadic wind investigation, which will be addressed in detail in subsequent chapter.

\subsection{Conclusions}

This study has developed a novel numerical framework that features co-working of the feedback forcing based immersed boundary (IB) approach, prediction-correction technique, and high-order compact finite difference scheme for three-dimensional simulations of moving bodies immersed in a fluid. The resulting code also exhibits a high level of parallelism to accommodate 
large-scale computations corresponding to three-dimensional complex flow scenarios.

As an important modification to the original Incompact3D package, the feedback forcing based immersed boundary method is embedded in the code to replace the direct forcing option, yielding significantly improved numerical stability in the simulation results. Also, to the authors' knowledge, no study was reported, prior to this study, on incorporating the prediction-correction scheme in immersed boundary method. As the first-time use of this coupling technique, both convergent acceleration and numerical accuracy have been demonstrated in the numerical experiments conducted in this study.

A number of benchmark cases were performed for examining the highly hybrid numerical simulation tools developed in the present study. The success in all the tests renders the code promising for its further applications to more complex problems, such as three dimensional tornado simulations, which will be presented in Part II and discussed in detail. 


\section{Chapter 6 A Novel Three-Dimensional Immersed-Boundary (IB) Approach Based Tornado Model Powered by Prediction-Correction Technique Part II - Tornadic Wind Simulations}

This chapter is based on the following paper (ready to submit to Journal of Computational Physics): Xixiong Guo, Jun Cao. A Novel Three-Dimensional Immersed-Boundary (IB) Approach Based Tornado Model Powered by Prediction-Correction Technique Part II - Tornado wind simulations

\subsection{Summary}

Based upon the previous work (Chapters 2 - 4) on tornadic wind induced loadings on buildings in the two-dimensional (2D) version, an extension from the stylized 2D tornado simulation to the more realistic three-dimensional (3D) version is conducted in this study. Recall that a novel hybrid framework coupling the feedback forcing immersed boundary method with the high-order compact finite difference scheme was developed and validated in Chapter 5, and that solver has been demonstrated to be an efficient and accurate simulation tool for complex fluid-structure interaction problems. Here, this framework is employed to investigate the interaction between tornado-like wind and building structure.

In the present 3D simulations, the "relative motion" principle is continuously employed, such that the original Rankine-combined vortex model is revised to an equivalent scenario in which the 
building is viewed as "virtually" translating in a "pinned" rotational flow field that remains time-invariant at the far field region. The logarithm based velocity profile joins the revised RCVM so that the boundary layer growth in the vertical direction is taken into account. This framework also relies on implementation of an enhanced degree of parallelism in the code architecture, such that the massive computation associated with the multiple variables in the turbulent N-S flow model over a large tornado evolution domain remains still affordable and also timewise acceptable.

The loadings induced by tornado in all three directions are studied by examining the coefficients of three components of the force and moment exerted on the building, respectively. Moreover, numerical investigations also apply to individual 3D segments resulting from sectioning the entire height of the building into a number of successive portions, and the loading coefficients are calculated on a segment-by-segment basis, aiming to look into the similitude of simulation results between a 3D segment and the stylized 2D layout. This detailed comparison can provide insights on correlation between $2 \mathrm{D}$ and $3 \mathrm{D}$ simulations, potentially helping the tornado dynamics research community save the computational expense by focusing more on $2 \mathrm{D}$ simulations.

\subsection{Three-dimensional Tornado Model}

Remark that real tornado field unfolds with velocity components in all three dimensions. However, the RCVM does not include any condition for the vertical velocity component, i.e. $w=0$. Instead, one extra term named $Z_{f}$ is applied into the velocity components in $\mathrm{x}$ - and $\mathrm{y}$ - directions, 
aiming to take the effects in vertical direction into account. Thus, in the light of RCVM, the resultant velocity components are expressed as:

$$
\vec{V}=u \vec{i}+v \vec{j}+w \vec{k}=Z_{f}\left[\left(V_{t x}-V_{\theta} \sin \theta\right) \vec{i}+\left(V_{t y}+V_{\theta} \cos \theta\right) \vec{j}+V_{t z} \vec{k}\right]
$$

where $\theta(x, y, t)=\tan ^{-1}\left(\frac{y-y_{0}-V_{t y} t}{x-x_{0}-V_{t x} t}\right) \cdot\left(x_{0}, y_{0}, z_{0}\right)$ denoting the initial position of the tornado center, and $\theta$ stands for the angle between the radial line passing through $(x, y, z)$ and the horizontal direction pointing to the right. Assuming that the translate motion only takes place in x-direction with the velocity fixed as $V_{t x}$, the velocity components can be easily derived as:

$$
\begin{gathered}
u= \begin{cases}{\left[V_{t x}-\left(y-y_{0}\right) \cdot \omega\right] Z_{f},} & r \leq r_{c} \\
{\left[V_{t x}-\left(y-y_{0}\right) \cdot r_{c}^{2} \omega / r\right] Z_{f},} & r>r_{c}\end{cases} \\
v= \begin{cases}{\left[\left(x-x_{0}-V_{t x} \cdot t\right) \omega\right) Z_{f},} & r \leq r_{c} \\
{\left[\left(x-x_{0}-V_{t x} \cdot t\right) r_{c}^{2} \omega / r\right) Z_{f},} & r>r_{c}\end{cases} \\
w=0
\end{gathered}
$$

Note that $Z_{f}$ is determined by the height from ground, $z$, according to the logarithmic law [21]:

$$
Z_{f}=\frac{u^{*}}{k} \ln \left(\frac{z+z_{0}}{z_{0}}\right)
$$

where $k$ and $z_{0}$ are constants, with $k=0.4$, and $z_{0}=0.00375$, respectively. $u^{*}$ is constant which can be pre-determined from the known $Z_{f}$ at known height. In this work, $Z_{f}$ is equal to one at $\mathrm{z}=1$. The computation is conducted upon Cartesian coordinates, and obviously the resultant velocity $\vec{V}$ is time-dependent.

As illustrated in Eq. (6.2), the time-dependent nature of the velocity in the customary RCVM 151 
model is basically due to its translational component. The "relative motion" concept is continually used here, in which the translation component is detached from the tornado and re-attached to the building construction at the same time, thereby creating a "virtual translation" of the buildings towards a "pinned", but purely rotating tornado. Therefore the resultant velocity turns to:

$$
\vec{V}=u \vec{i}+v \vec{j}+w \vec{k}=Z_{f}\left[\left(-V_{\theta} \sin \theta\right) \vec{i}+\left(V_{\theta} \cos \theta\right) \vec{j}\right]
$$

where $\theta(x, y)=\tan ^{-1}\left(\frac{y-y_{0}}{x-x_{0}}\right)$. Eq. (6.4) can be rewrote as:

$$
\begin{gathered}
u= \begin{cases}{\left[-\left(y-y_{0}\right) \cdot \omega\right] Z_{f},} & r \leq r_{c} \\
{\left[-\left(y-y_{0}\right) \cdot r_{c}^{2} \omega / r\right] Z_{f},} & r>r_{c}\end{cases} \\
v= \begin{cases}{\left[\left(x-x_{0}\right) \omega\right) Z_{f},} & r \leq r_{c} \\
{\left[\left(x-x_{0}\right) r_{c}^{2} \omega / r\right) Z_{f},} & r>r_{c}\end{cases} \\
w=0
\end{gathered}
$$

Consequently, the boundary condition after re-interpreted RCVM becomes stationary, leading to a significantly improved maneuverability in computational framework establishment. Thus, the tedious time-dependent nature of this RCVM-based tornado model in three dimension is re-tailored to be an entirely time-independent, and manageable model.

\subsection{Numerical methods and boundary condition}

\subsubsection{Computational geometry and initial parameters setup}

Recall from Section 5.3, the proposed framework was conducted and adapted on the basis of an 
open-source package Incompact3D [69]. In this part, the same methodology was applied for tornado cases investigation.

In all present cases in this work, unless otherwise specified, the computational domain is fixed as a rectangular with the domain size of $8.5 \times 4 \times 3.5$ in the $x$-, $y$-, and $z$ - direction respectively. A uniform mesh with the size of $545 \times 257 \times 225$ is taken. The eligibility of mesh resolution will be examined in Section 6.4.1. After imposing the re-interpreted RCVM, the counterclockwise rotating vortex is pinned at the centerline of the domain, with the obstacle travels along the $x$-axis with a constant velocity. The proposed feedback forcing IBM embedded Incompact3d framework will be applied to calculate the tornado-induced loadings for circular cylinder and square prism building. Schematics of the building constructions are shown in Fig. 6.1(a) and (b), respectively. The side length of square prism $D_{s}$ is equal to 0.25 , which is considered as the characteristic length, and the diameter of cylinder $D_{c}$ is fixed as $\frac{1}{2 \sqrt{\pi}}$. Thus, the projection area $\left(A_{z}\right)$, volume of the two building structures are the same with the same height $H$. The height of each building $(H)$ varies from 0.5 to 1.75 in different test cases, which will be specifically clarified by cases. The centerline of the building should be originally placed at a reasonable distance from the boundary of the domain. At this work, the initial position is $(7,2)$, as illustrated in Fig. 6.2, then the building "virtually" translates along x-direction with a constant velocity $\vec{U}_{T}=-1.0$. 


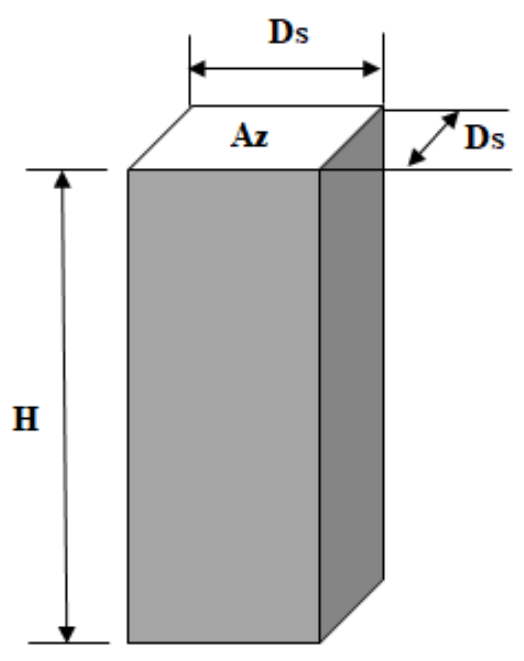

(a)

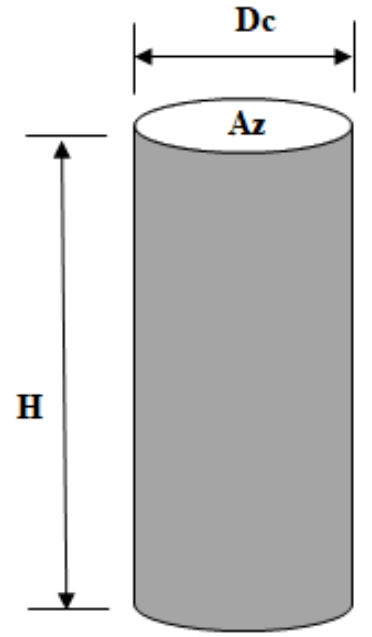

(b)

Fig. 6.1 Geometry and nomenclature of testing buildings:

(a) square prism (b) circular cylinder

\subsubsection{Boundary conditions}

As depicted in Fig. 6.2, in present framework, unless otherwise specified, all boundaries are given by Dirichlet boundary condition. More concretely, no-slip boundary condition $\left(u=u_{\text {wall }}=0\right)$ is employed for all surfaces of solid immersed obstacle and bottom surface (4) of the domain. Velocity profile at the boundary surfaces determined by the definition of re-interpreted RCVM (Eq. (6.5)) are given to all other boundaries at first (1), (2), (3), (5) and (6)).

Additionally, as the height of immersed obstacle is finite, additional mean velocity profile is added to two transverse surfaces (5) and (6), as well as left, right and top surfaces (1), (2) and (3)), so that the thickness of boundary layer is properly considered, and the continuous of velocity to all directions can be guaranteed. The mean velocity profile is also determined by logarithmic law 
[21], as shown in Eq. (6.3). However, since a translational velocity is imposed to the building in the domain, the velocity profile need to be customized accordingly to adapt this situation. Therefore, in Fig. 6.3, the velocity at each z plate can be determined as $U(\mathrm{z})+\left(-U_{T}\right)$ after taking boundary layer effect into consideration. Note that the velocity at the bottom boundary $(z=0), u=-U_{T}$, which means the boundary layer effect $U(\mathrm{z})$ is zero at this surface, and only translational velocity is taken into account.

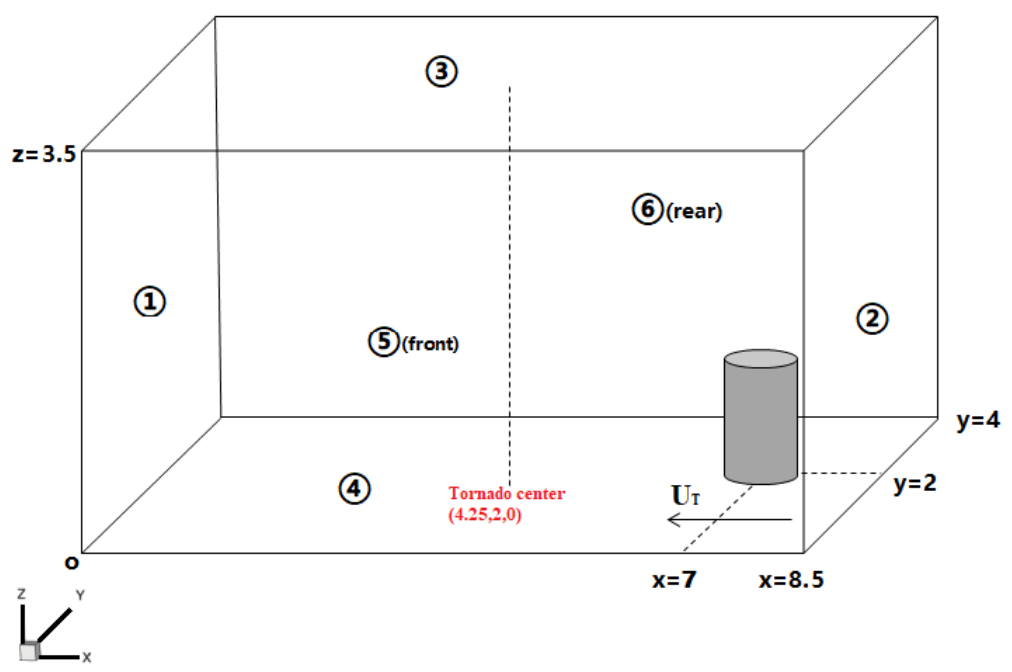

Fig. 6.2 Schematic and dimensions of the domain

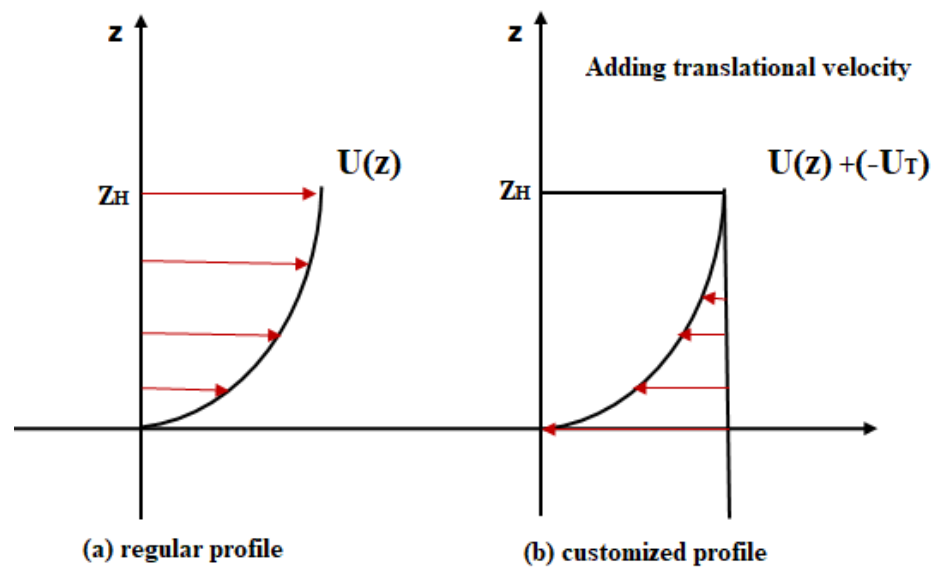

Fig. 6.3 Boundary layer profile treatment 


\subsection{Results and analyses}

Numerical simulations on how re-tailored RCVM described tornado model affects a three-dimensional building structure were performed and analyzed in details at $\operatorname{Re}=1000$. Although the feedback-forcing based framework has been validated extensively in Section 5.4 at a relatively low Reynolds number, its reliability and mesh resolution still need to be further examined at $\operatorname{Re}=$ 1000 before it implements in tornado-induced flow investigations. Therefore, flow past an infinite-length circular cylinder at $\mathrm{Re}=1000$ will be studied at first.

\subsubsection{Flow over an infinite-length circular cylinder at $\mathrm{Re}=\mathbf{1 0 0 0}$}

A mesh independent study is conducted here. Two different mesh resolutions are designed: One is the mesh size described in Sec. 6.3.1, $\Delta x=\Delta y=\Delta z=0.015625$, namely current mesh in Table 6.1, and the other refined mesh in Table 6.1 is defined as $\Delta x=\Delta y=\Delta z=0.01$. Boundary conditions are set as described in Section 5.3.7 under the condition that the height of immersed obstacle is equal to domain height. In Table 6.1, the mean drag coefficient $\overline{C_{d}}$, and the root-mean-square (RMS) value of lift coefficient $C_{l}^{\prime}$ is measured, together with the Strouhal number. Fig. 6.4 and Fig. 6.5 show the three-dimensional vorticity iso-surface contours from front view and top view, respectively. It has been observed that the vortex shedding phenomenon is obvious, and the turbulence flow is gradually generated. Meanwhile, shear layer instabilities happen, which conversely intensifies the transition of the flow into turbulence. Another significant evidence 
of three-dimensional effect in this example is the streamlines plotted in Fig. 6.6. Streamline patterns captured from two different plates $(\mathrm{z}=1$ and $\mathrm{z}=3$ ) appear to be distinctly different, which indicates the flow characteristics in each plate are not the same. This again proves the existence of three-dimensional effect during the flow evolution. Furthermore, the average drag coefficient $\bar{C}_{d}$, root mean square lift coefficient $C_{l}^{\prime}$ and Strauhal number St are measured and compared with existing references in Table 6.1. All above quantities are within the range of the references. The difference between the current mesh and refined mesh are almost negligible. Other than the comparable force coefficients, no streamline penetration across the boundary of the immersed object in Fig. 6.6, confirming the no-slip boundary condition on the object surface is strictly guaranteed. To summarize, mesh resolution defined in Section 6.3.1 is adequate and reliable enough at $\operatorname{Re}=1000$ for further investigations.

Table 6.1 Mesh dependent check on flow over infinite cylinder at $\operatorname{Re}=1000$

\begin{tabular}{|c|ccc} 
& Mean drag coefficient $\overline{C_{d}}$ & RMS lift coefficient $C_{l}^{\prime}$ & Strouhal number St \\
Current mesh & 1.11 & 0.309 & 0.201 \\
Refined mesh & 1.14 & 0.332 & 0.210 \\
\hline Zhao et al. [97] & 1.17 & 0.335 & 0.210 \\
\hline Lei et al. [98] & 1.11 & $0.30 \sim 0.35$ & 0.200 \\
\hline Mittal [99] & 1.15 & - & 0.200
\end{tabular}




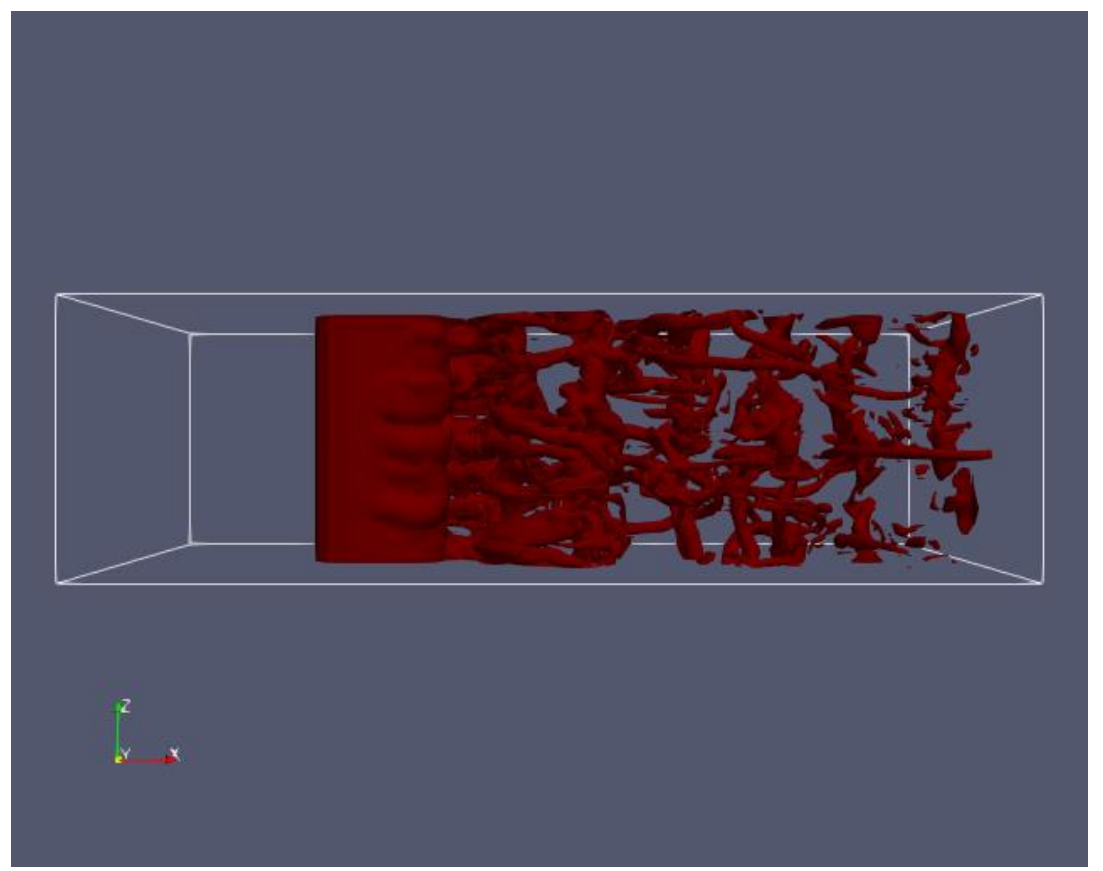

Fig. 6.4 Vorticity (vorticity =1) iso-surface from front view

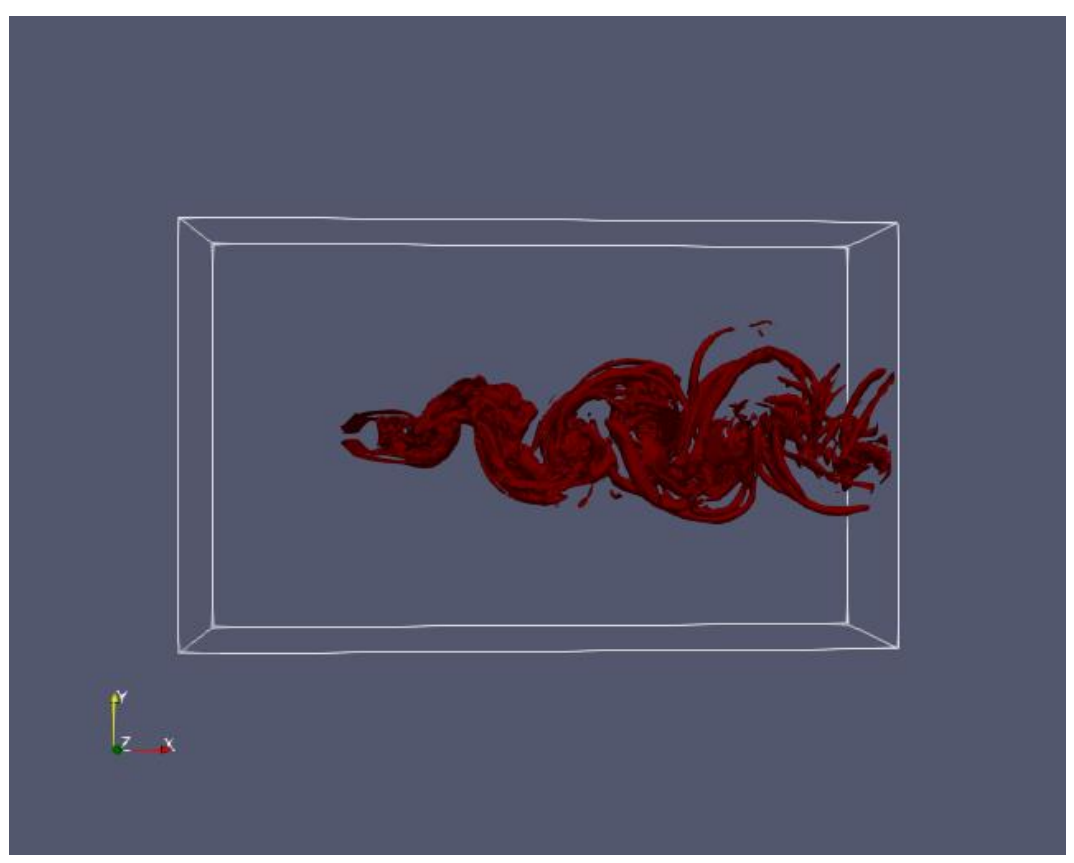

Fig. 6.5 Vorticity (vorticity =1) iso-surface from top view 


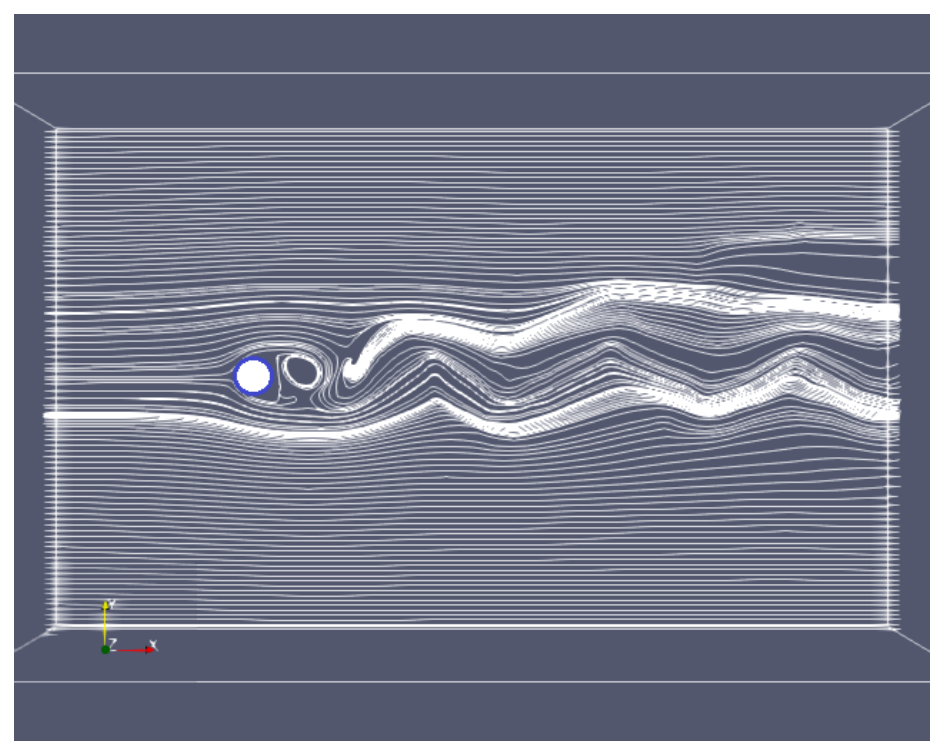

(a)

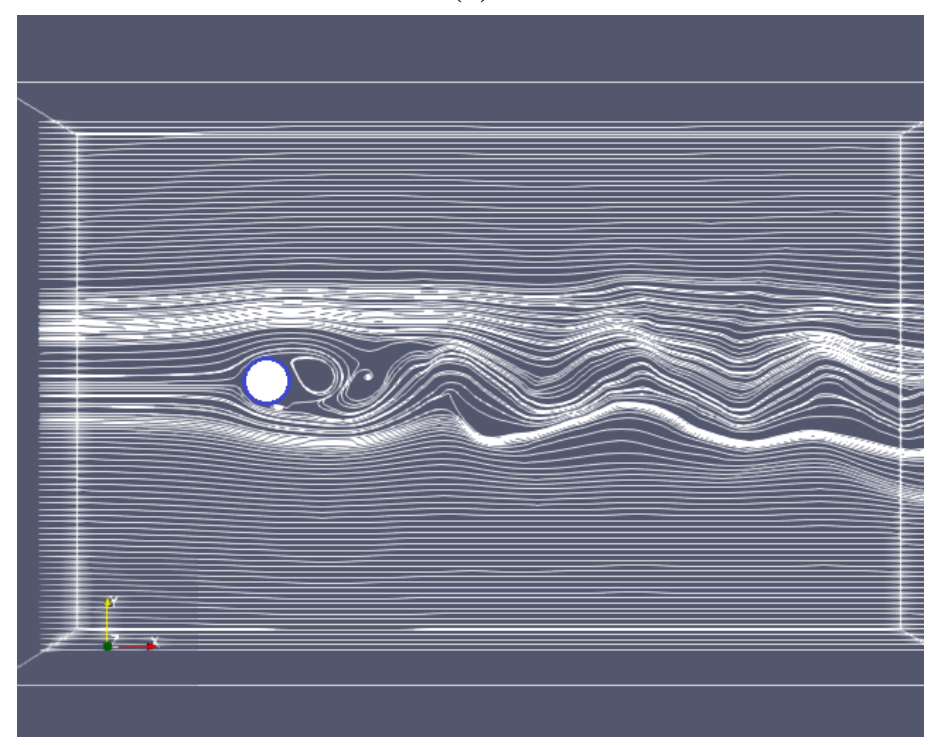

(b)

Fig. 6.6 Streamline from (a) $z=1$ plate (b) $z=3$ plate

\subsubsection{One benchmark case study}

After extensive validation, the aforementioned RCVM, as well as its corresponding boundary condition is imposed onto the computational domain, standing for tornado-like wind, and then a 
three-dimensional circular cylinder with the height $H=0.75$, representing a single building structure, is initially placed to the right side of domain as a benchmark case discussed in this sub-section.

For this type of flow, the forces acting on the building are calculated by integrating the force density obtained from IBM, and the moments are determined with the aid of force and its corresponding force arm. Then, the force and moment coefficients on the building [100] for all three directions are expressed in terms of

$$
\begin{gathered}
C_{x}=\frac{2 F_{x}}{\rho U_{T}^{2} S_{x}} \\
C_{y}=\frac{2 F_{y}}{\rho U_{T}^{2} S_{y}} \\
C_{z}=\frac{2 F_{z}}{\rho U_{T}^{2} S_{z}} \\
C_{M_{x}}=\frac{2 M_{x}}{\rho U_{T}^{2} S_{y} H} \\
C_{M_{y}}=\frac{2 M_{y}}{\rho U_{T}^{2} S_{x} H} \\
C_{M_{z}}=\frac{2 M_{z}}{\rho U_{T}^{2} S_{z} H}
\end{gathered}
$$

where $F_{x}, F_{y}$ and $F_{z}$ are the loading forces in $x$-, $y$-, $z$-directions, respectively. $M_{x}, M_{y}$ and $M_{z}$ are the moment and $S_{x}, S_{y}$ and $S_{z}$ denotes the projection area in $x$-, $y$ - and $z$-directions. $H$ is the height of building, and $U_{T}$ is the "virtual" translational velocity of the building.

Fig. 6.7 and Fig. 6.8 group the velocity magnitudes from horizontal (top-view) and vertical 160 
(front-view) plane at four representative time instants, including initial condition, before "core-in" instant at pre-interaction stage , primary stage, and after "core-out" instant at post-interaction stage. As demonstrated in Fig. 6.7(a), the initial condition of the velocity magnitude contour from top view satisfies the RCVM governing equation Eq. (6.5), and the velocity magnitude increases from the tornado center and reaches a maximum value at a critical radius $r=r_{c}$ and then decreases when it is away from the center. From the side view (Fig. 6.8 (a)), the maximum velocity magnitude keeps increase when the height (z) goes up, which can be easily explained again by Eq. (6.5). As $Z_{f}$ is proportional to the height, the maximum velocity determined by $Z_{f}$ undisputedly keeps the same pattern here. When the building starts to "virtually" move towards the tornado center, namely in pre-interaction stage, as shown in Fig. 6.7 (b) and Fig. 6.8 (b), the rotational flow pattern seen from top view overall retained except in the vicinity of the cylinder. This indicates that the tornado-construction interaction emerges, but has not been significant yet. The affecting region mainly located in the vicinity of the cylinder. The velocity magnitudes at the influenced zone are larger when comparing to initial condition. Similar observation can be found from the side view in Fig. 6.8 (b). Moreover, the higher velocity at the bottom of plane can be explained by a downwash flow induced by the three-dimensional effect of RCVM tornado model. The strong effect of downwash on the flow structure can be transformed to an increased suction loading force in $z$-direction, which will be discussed later. After the construction enters the inner core region, namely in primary stage, the interaction was strongly distorted by the complex tornado. Several small 
eddies is observed around the cylinder, but without obvious regularity. By comparing Fig. 6.7(b),(c) and Fig. 6.8(b), (c), the highest velocity is found in front of the cylinder in the pre-interaction stage; then, in the primary stage, the zone was spread to the surrounding areas of the cylinder, and performs as several disordered small eddies (as shown in Fig. 6.9(b)). Moreover, from side view, the downwash flow at the bottom of the domain still exists in the primary stage, which attributes a suction force in $z$-direction. With time elapses, the velocity magnitude around the building and in the domain restore to a relatively mild status when tornado goes more distant from the building after post-interaction stage (Fig. 6.7(d) and Fig. 6.8 (d)). As shown in previous chapters, translational component dominates the flow in the pre-interaction stage, while rotational component makes a bigger impact during the primary stage, and the dominant translational flow re-occurs at the post-interaction stage in the two-dimensional simulation. This conclusion still stands in horizontal plane in present case, as shown in Fig. 6.7(a)-(d). However, taking the velocity in $z$-direction into consideration, as referred in Fig. 6.8(a)-(d), force in $z$-direction, which can be attributed to the downwash flow, plays a critical role in the flow. Whether the suction force in z-direction dominates the flow needs to be further investigated by quantitative analysis in the following discussion. 


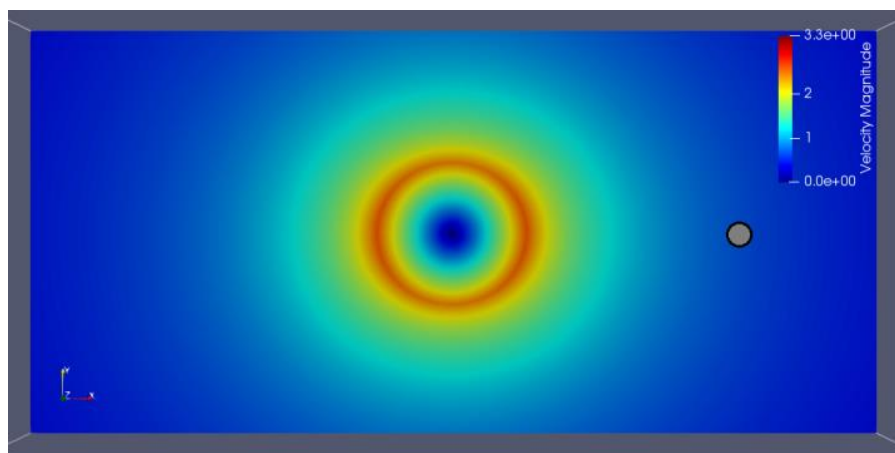

(a)

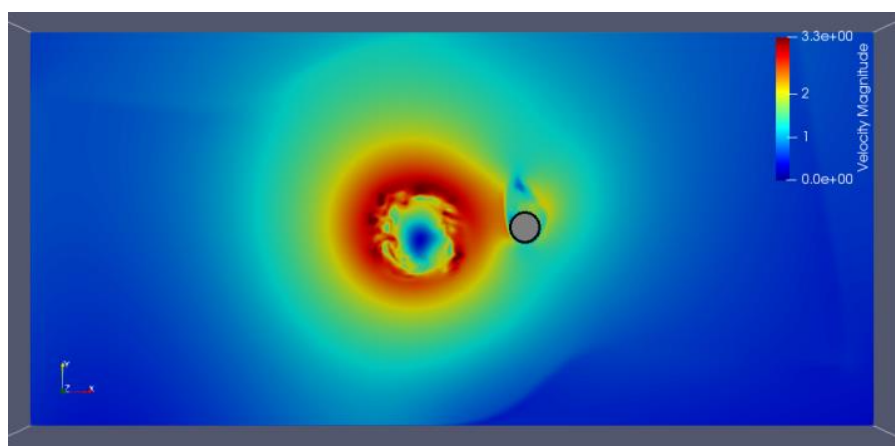

(b)

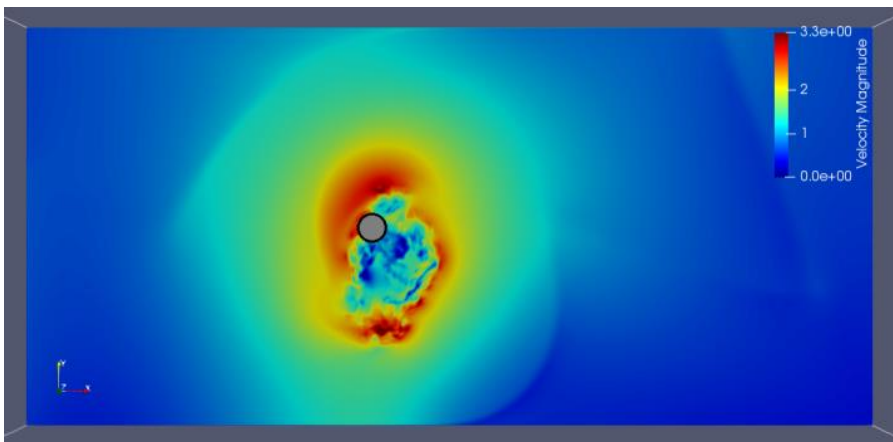

(c)

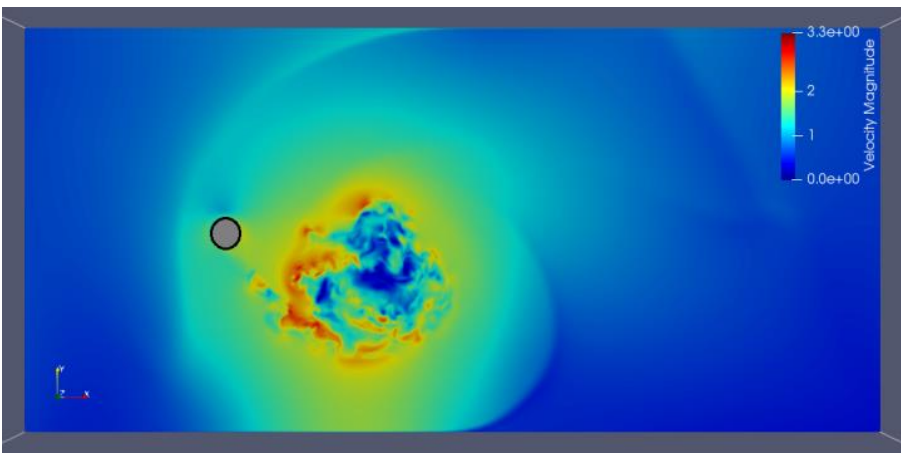

(d)

Fig. 6.7 Velocity magnitude in horizontal plane (top-view, $\mathrm{z}=0.75$ ):

(a) Initial condition; (b)Pre-interaction stage (c) Primary stage (d) Post-interaction stage 


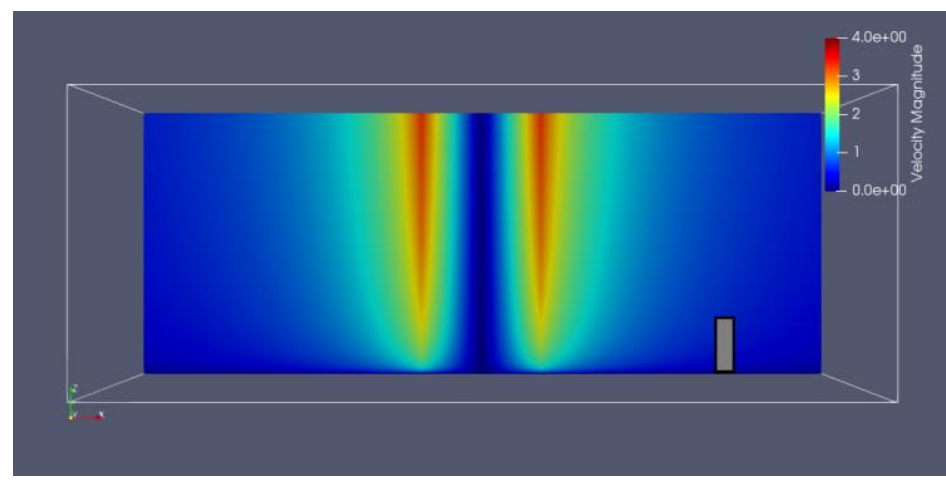

(a)

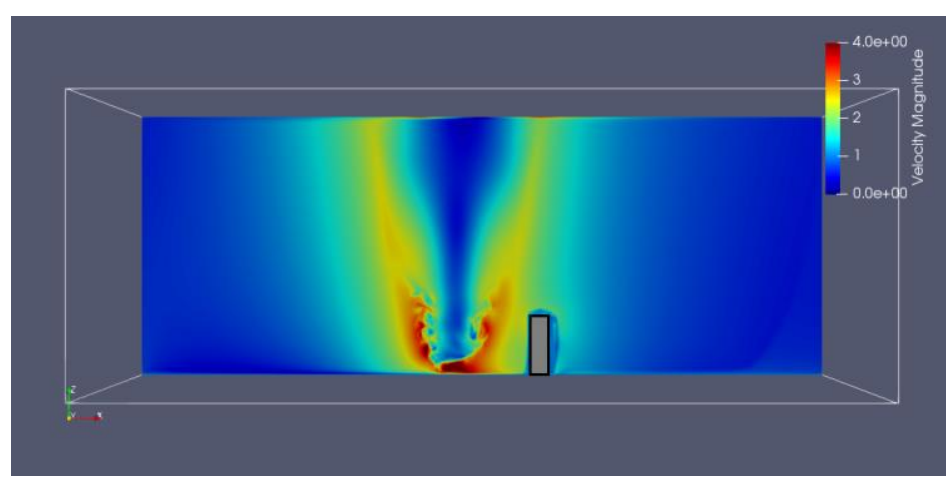

(b)

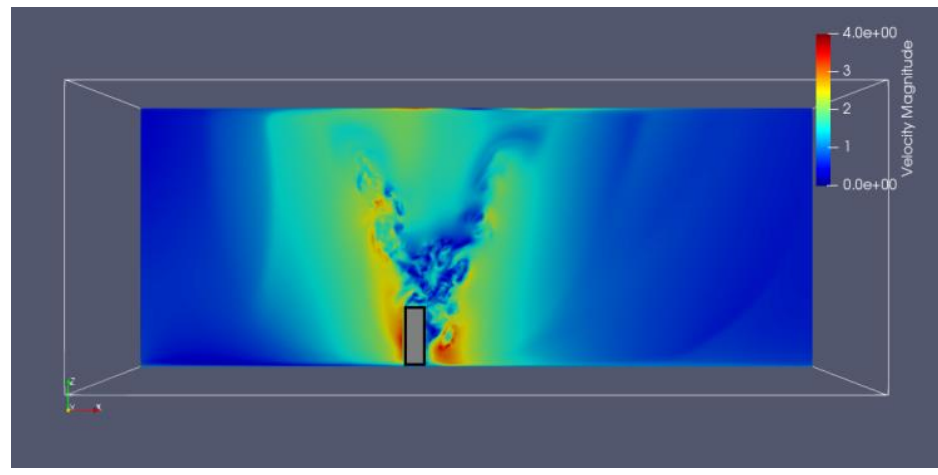

(c)

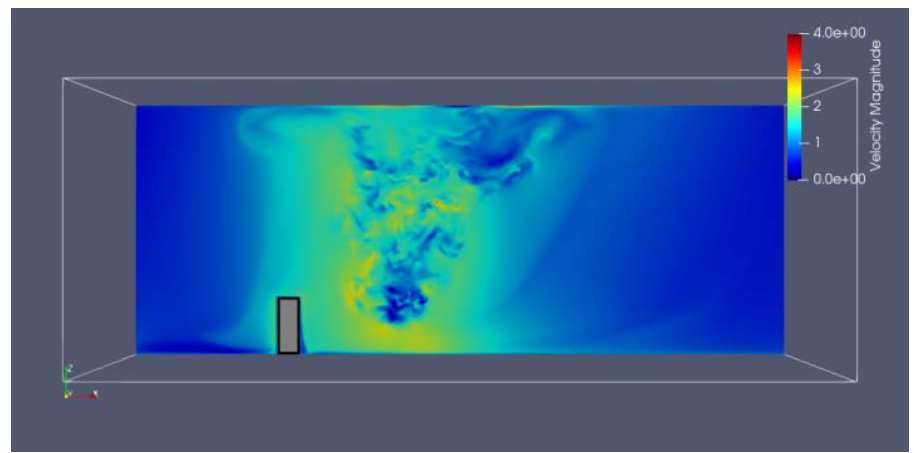

(d)

Fig. 6.8 Velocity magnitude in vertical plane (front-view, y=2): (a) Initial condition; (b) Pre-interaction stage (c) Primary stage (d) Post-interaction stage 


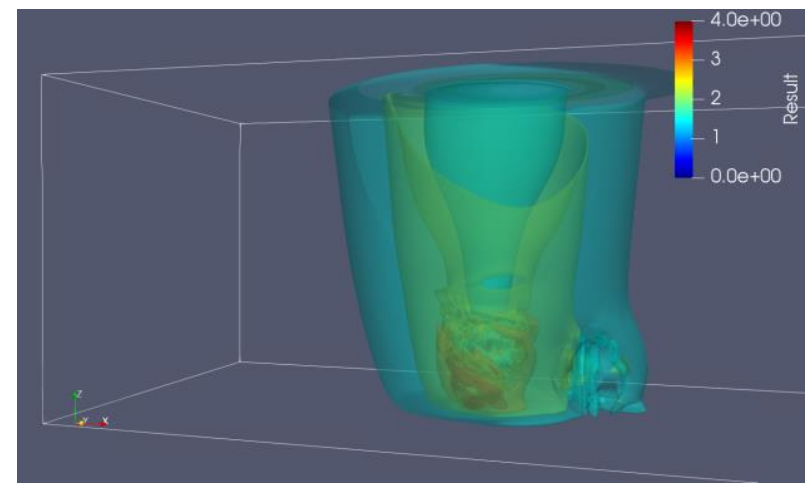

(a)

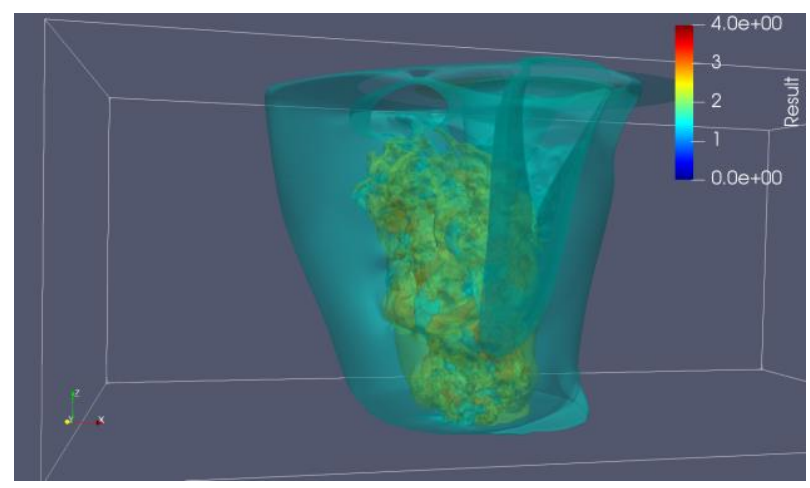

(b)

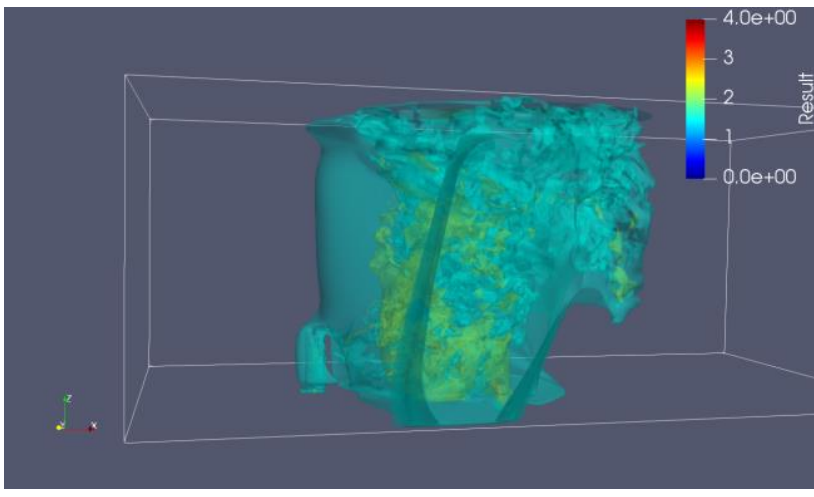

(c)

Fig. 6.9 Velocity magnitude contour: (a) Pre-interaction stage (b) Primary stage (c) Post-interaction stage

Besides the qualitative analysis based on the velocity magnitude contours captured from different perspectives, quantitative analysis through evolution of force and moment coefficients in all three directions, as defined in Eqs. (6.6) - (6.11), can be also examined. The curves of $C_{x}, C_{y}, C_{z}$, $C_{M x}, C_{M y}$ and $C_{M z}$ corresponding to $\mathrm{Re}=1000$ are grouped in Fig. 6.10 and Fig. 6.11, respectively. 
In these figures, position $x=0$ on the horizontal axis represents the time instant at which the centers of the tornado and cylinder are nominally coinciding if solely taking into account the translation of the tornado center; in the intervals $x<0$ and $x>0$, the tornado is approaching and leaving the cylinder, respectively. In pre-interaction stage $(x<-0.75)$, all curves, including force and moment coefficients remain relatively stable, which indicate the tornado-construction interaction is moderately mild at this stage. On the other hand, at around $x=0, C_{z}$ starts to precede $C_{x}, C_{y}$ and tops $C_{x}, C_{y}$ in primary stage which implies the dominance of suction force in z-direction, and the extremum value of $C_{z}$ is found to be higher than $C_{x}, C_{y}$. First major turning point approximately locates at $x=-0.8$ (close to the "core-in" time), after which $C_{y}$ starts to decrease. The primary interaction stage nominally refers to the range of $-0.75<x<0.75$ in present work. During this time period, $C_{x}, C_{y}$, and $C_{z}$ reach their extremum points respectively, while all moment coefficients $C_{M x}$, $C_{M y}$ and $C_{M z}$, experience dramatic fluctuation. All these variations indicate the fierce interaction happening in this stage. $C_{z}$, which is $50 \%$ larger than $C_{x}, C_{y}$ (as shown in Fig. 6.10), indicates that suction force in z-direction still dominates the flow field. Another significant observation in this case is the fierce fluctuation of the moment coefficients, as depicted in Fig. 6.11. It can be further interpreted as the construction endures twisting dynamically from all directions, which is very dangerous and vulnerable. The amplitude of the moment coefficients reach their extremum values during primary stage as well, and $C_{M z}$ dominates the field in most time of pre-interaction and primary stages (exceptional for $-0.6<x<-0.3$, where $C_{M x}$ tops $C_{M z}$ ). After interpreting the forces 
and moments covering all stages, it can be concluded that the devastation of tornado is not only caused by the tremendous loadings, but also attributed to unpredictable twisting in either direction.

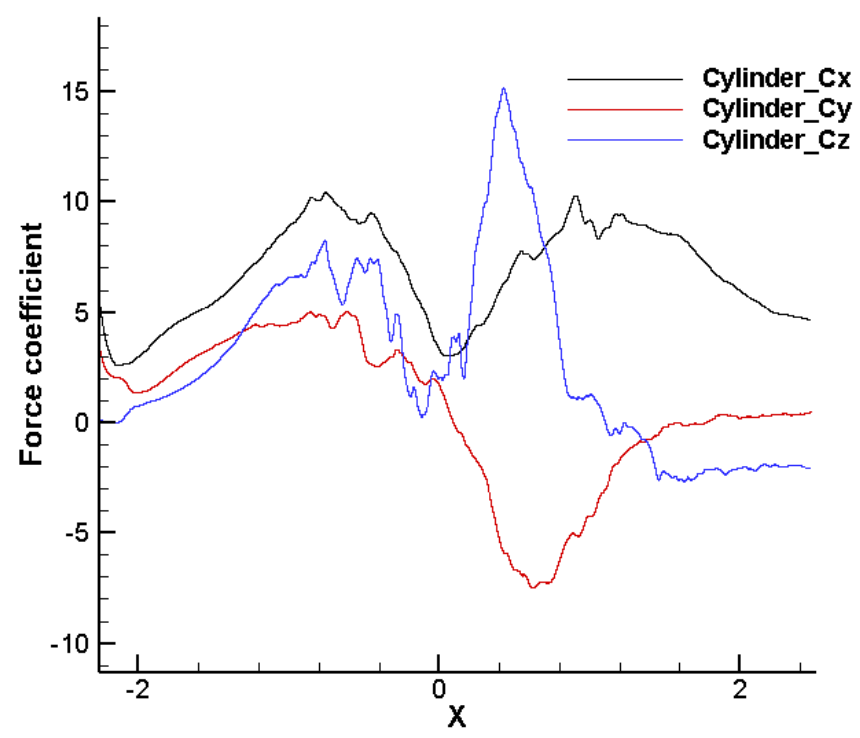

Fig. 6.10 Force coefficients evolution on cylinder with $\mathrm{H}=0.75$

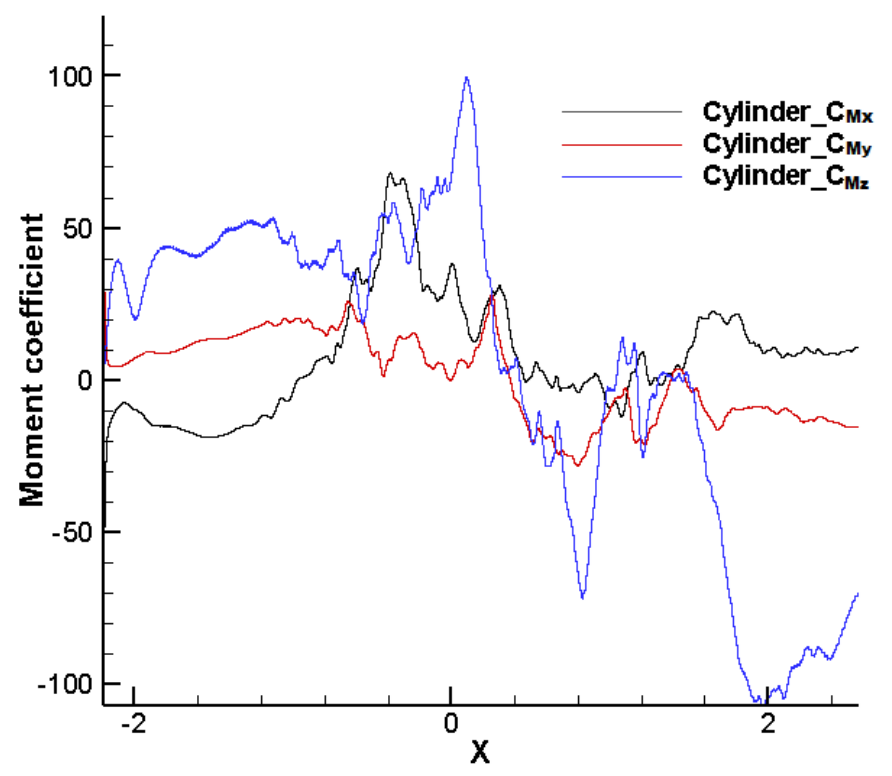

Fig. 6.11 Moment coefficients evolution on cylinder with $\mathrm{H}=0.75$ 


\subsubsection{Quantitative analysis of loadings on different heights}

There are several factors that can affect the loading performance of one specific building when tornado sweeps over it. One of them should be the height of the building. In order to analyze how building height can influence the total loadings, four different height $(H=0.5,0.75,1.0,1.75$, respectively) with the same enclosure outlook are conducted and the forces and moments are calculated and compared as follows.

Firstly, the circular cylinder buildings with different height are discussed. It can be found that the evolutions of force coefficients exhibit similar patterns in four different heights, as depicted in Fig. 6.12. Three major turning points are observed for all cases approximately at the "core-in", "coincide" and "core-out" time instants from $C_{x}$ evolution, which again indicates the force coefficients undergo significant elevation in the primary stage for all heights. On the other hand, the dramatic fluctuation on moment coefficients in Fig. 6.13 reveal the strong twisting happens at the same time. All these characteristics, which has been discussed in last sub-section, repeatedly confirm the destruction of tornadic wind in real life.

Although the extremum loadings in $x$ directions corresponding to different heights are relatively close, a clear trend still can be concluded that lower height building surprisingly has higher maximum loadings in $x$ and $y$ directions. However, the maximum loading in $z$-direction increases as the height increases (exclude $\mathrm{H}=1.75$ situation, which will be explained in Section 6.4.5), which 
underlies that the suction force has larger impact on the higher structure. More than $50 \%$ growth is observed for the peak value of $C_{z}$, when $H$ increases from 0.5 to 1.0. Again, the loadings in $z$-direction are apparently higher than those in other two directions, which proves the devastating influence of the vertically induced by tornadic wind.

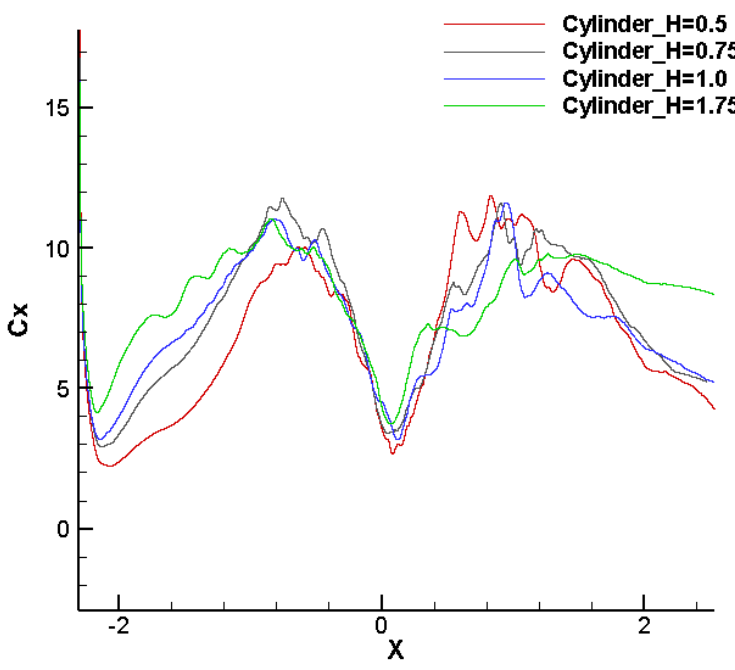

(a)

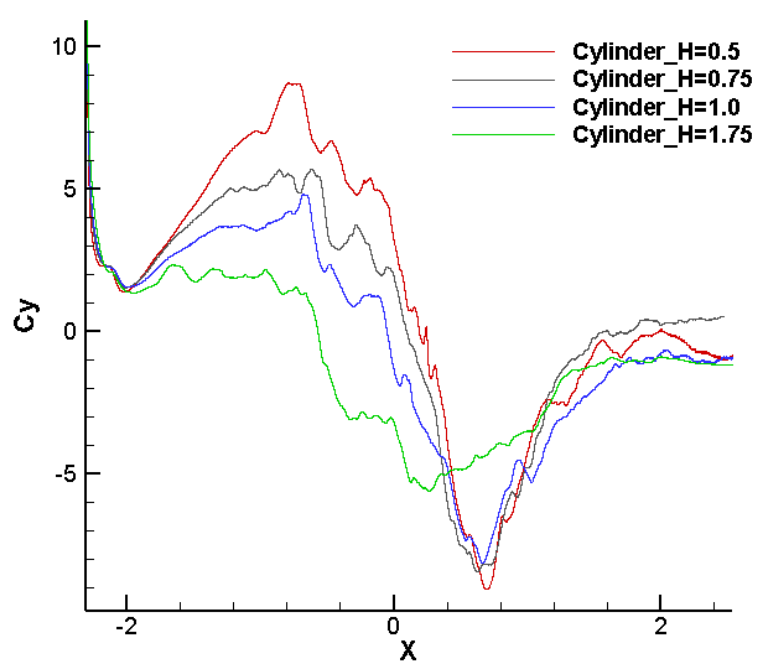

(b)

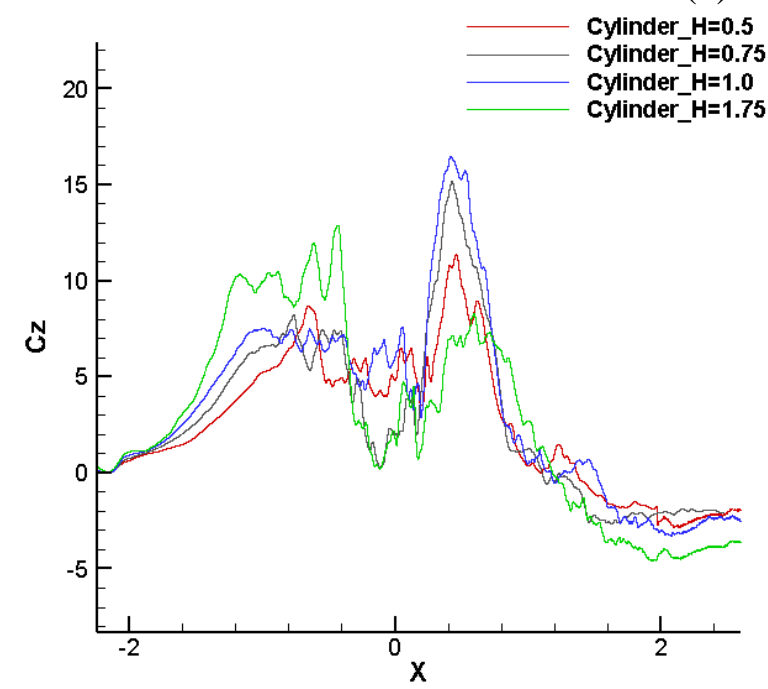

(c)

Fig. 6.12 $C_{x}, C_{y}, C_{z}$ evolutions on cylinder for different heights

Likewise, when considering the maximum value on moment coefficients in $x$ - and $y$-direction 
$C_{M_{x}}, C_{M_{y}}$ (see Fig. 6.13), no apparent relation is detected. As the moment coefficients are determined by multiple factors, including force and its corresponding force arm, the trends of moment coefficient are much more complex and difficult to make a conclusion at this level.

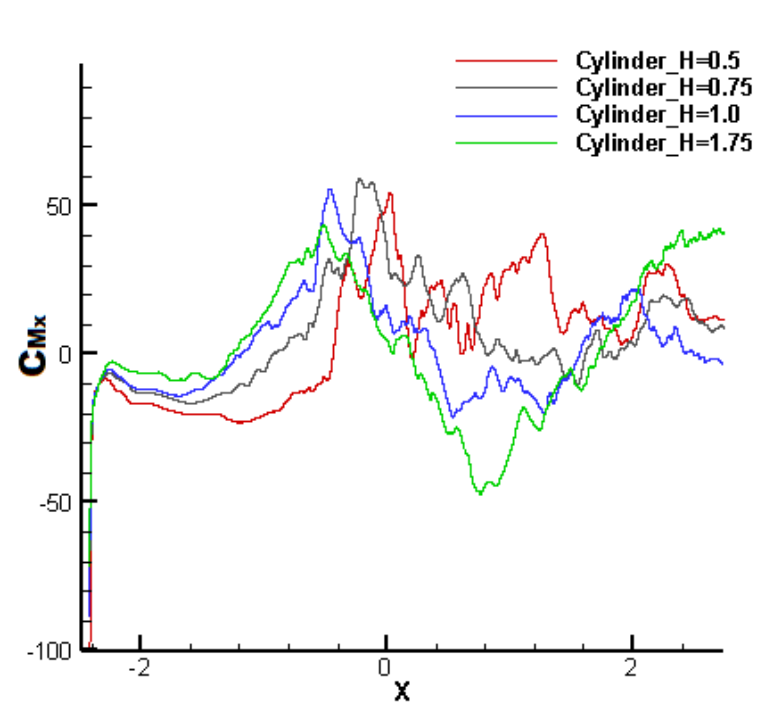

(a)

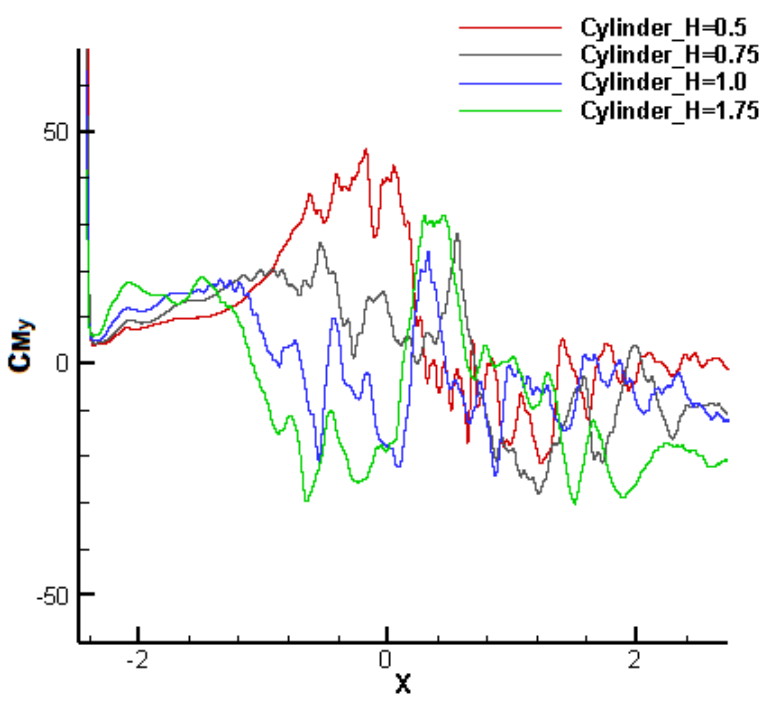

(b)

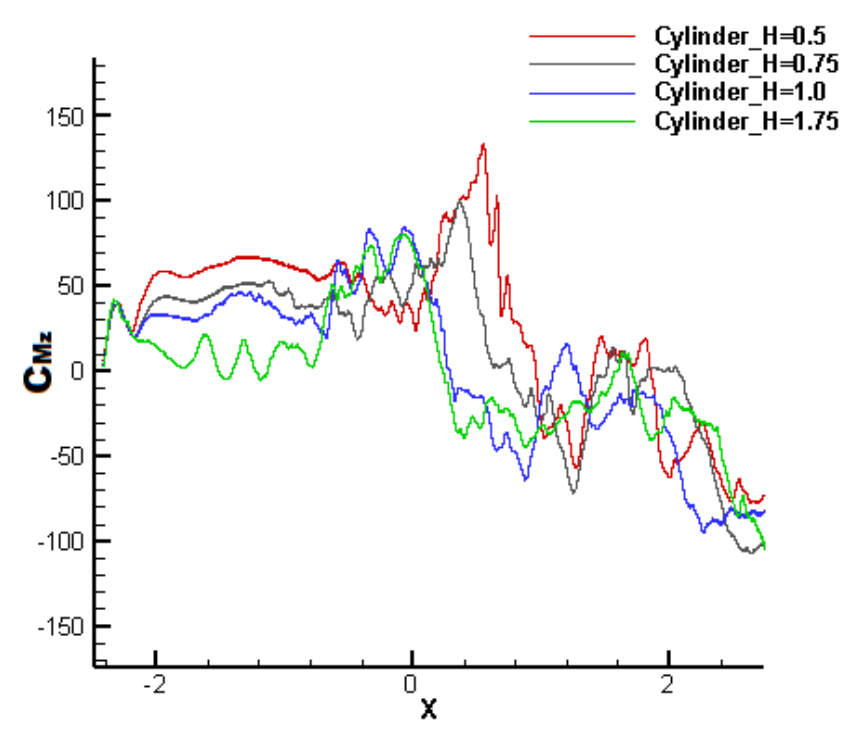

(c)

Fig. $6.13 \mathrm{C}_{\mathrm{Mx}}, \mathrm{C}_{\mathrm{My}}, \mathrm{C}_{\mathrm{Mz}}$ evolutions on cylinder for different heights

However, the moment coefficient in $z$-direction $C_{M_{z}}$ can be found much higher than that in 170 
other two directions from Fig. 6.13, in lower structures, as the moment $M z$ is determined by forces in $x$ and $y$ directions, which perform higher magnitude in lower structures. In summary, structures with lower height tend to be dominated by $C_{M_{z}}$, and $C_{M_{z}}$ is likely to reach a larger magnitude in structures, comparing to $C_{M_{x}}$, and $C_{M_{y}}$.

When it comes to the same type of tornado past a square prism, the patterns aforementioned above still hold. As shown in Fig. 6.14, force coefficients $C_{x}, C_{y}$ decrease as the height of prism $H$ increases, and $C_{z}$ exhibits the largest magnitude in $H=1.0$ case. No clear relation can be obtained regarding to the moment coefficients $C_{M_{x}}, C_{M_{y}}$ and $C_{M_{z}}$, in Fig. 6.15.

In summary, the increase of construction height $H$ leads to elevated wind suction force in z-direction induced by RCVM described tornado, when $H$ is far less than the height of domain. However, there is no clue to determine how $H$ affects the force moment of each construction, which can be attributed to the expression of moment, mutually determined by force and corresponding force arm. Notably, force and moment coefficients in $z$-direction are far larger than those in $x$-, and $y$ - directions, and these again reveal the dominance of forces and moments in vertical direction. This conclusion is proved to be valid in all heights and shapes in present study. 


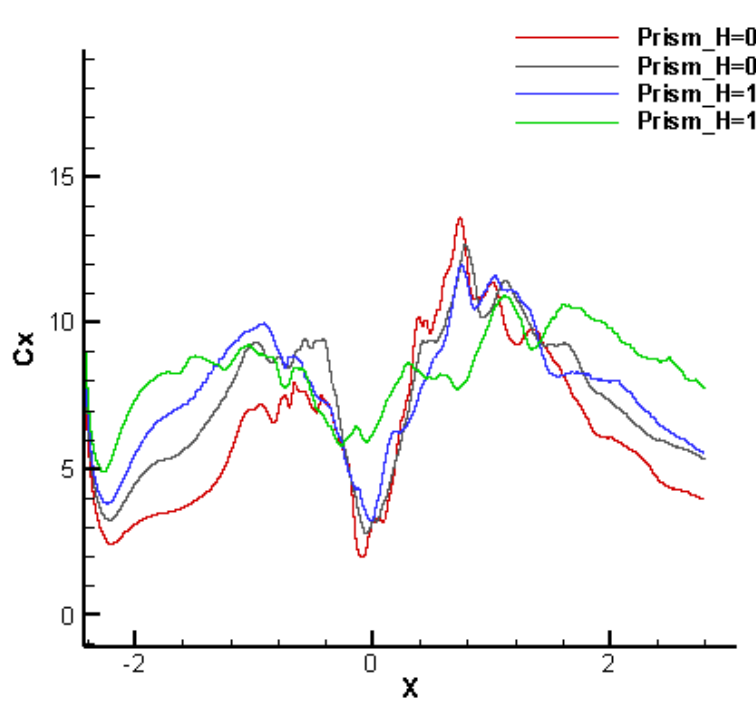

(a)

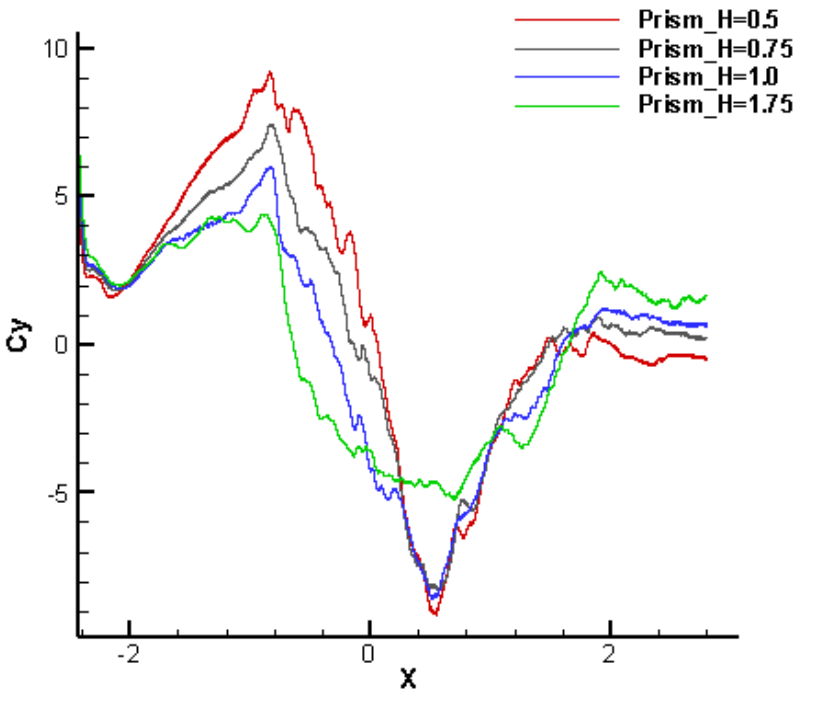

(b)

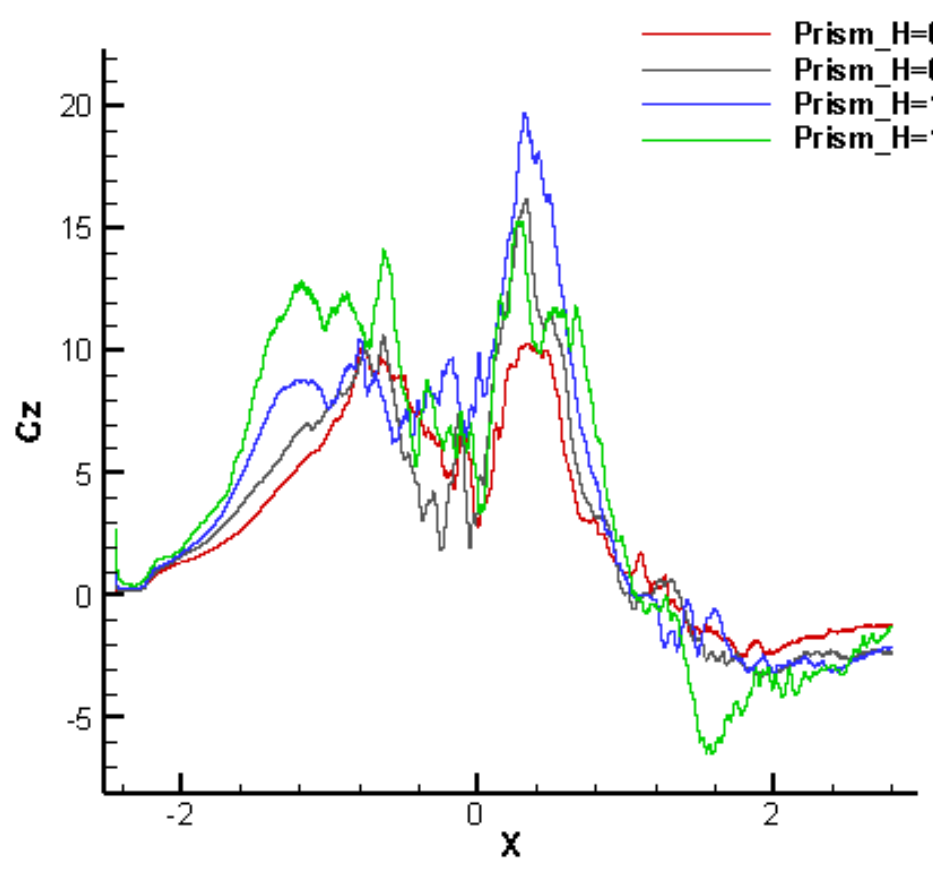

(c)

Fig. $6.14 C_{x}, C_{y}, C_{z}$ evolutions on prism for different heights 


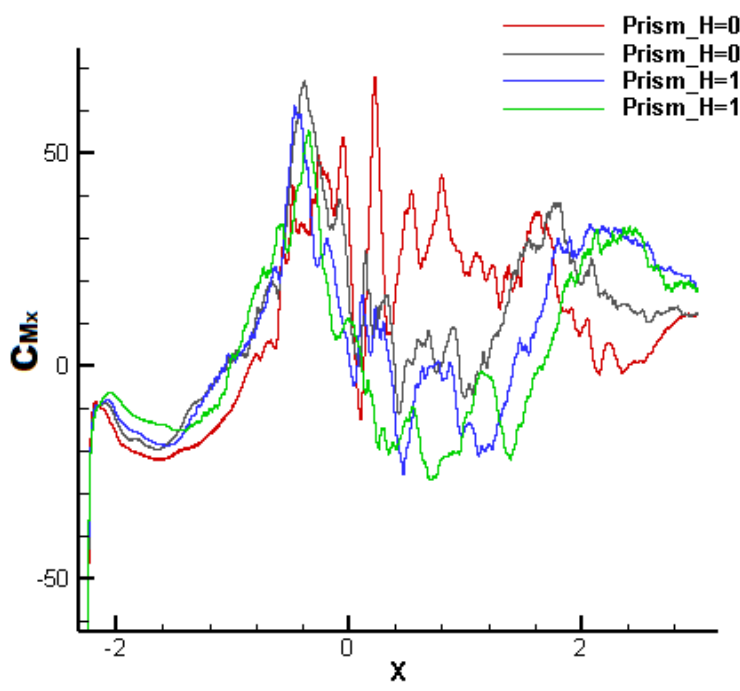

(a)

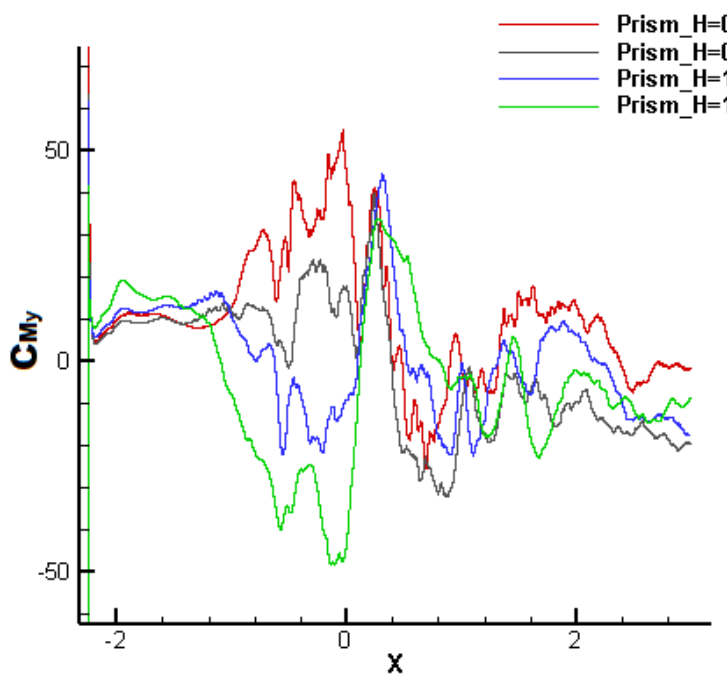

(b)

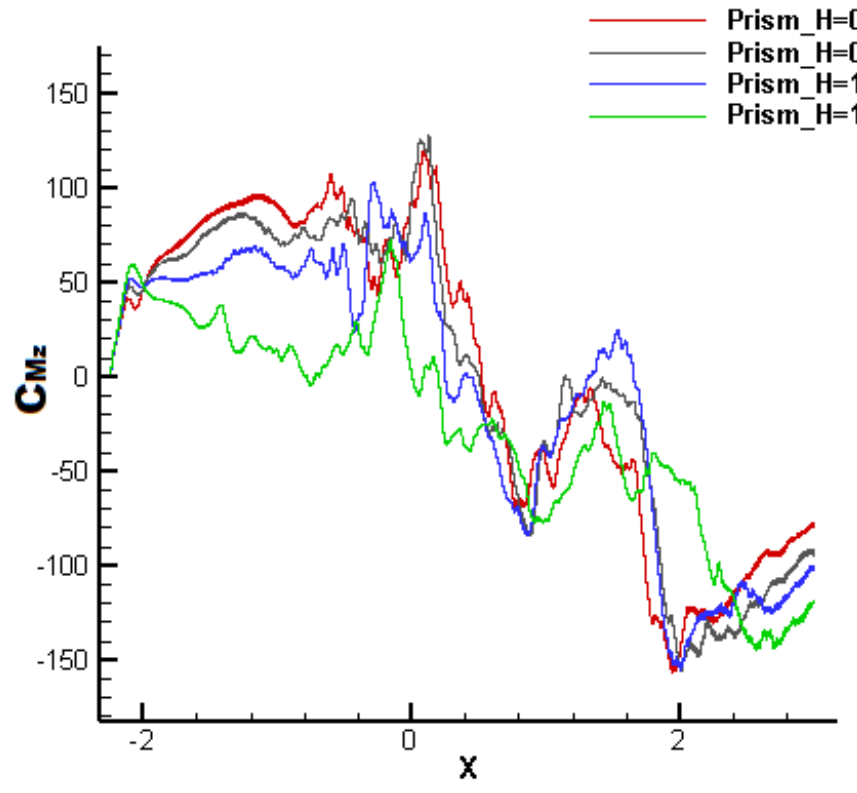

(c)

Fig. 6.15 $\mathrm{C}_{\mathrm{Mx}}, \mathrm{C}_{\mathrm{My}}, \mathrm{C}_{\mathrm{Mz}}$ evolutions on prism for different heights

\subsubsection{Quantitative analysis between tornadoes over a prism and over a cylinder}

Except for the height of building structure, another influence factor discussed in present work is the shape of the building. It is well known the flow features, such as shape of weak vortex, shedding 
vortices, and streamlines can be varied, when the flow over objects with different shapes. Correspondingly, the force coefficients and moment coefficients should be also significantly affected by the shape of building when tornado-like wind past over it, and this topic will be discussed as follows. As introduced in Section 6.4.3, two different types of building: a circular cylinder and a square prism, with the same cross-section area, are initially imposed as the obstacle, separately in the work.

As shown in Fig. 6.16, when the height of the structure $H=0.5$, the force coefficient $C_{x}$ of prism is slightly larger than that obtained from circular cylinder, $C_{y}$ for both cases are nearly the same. While $C_{z}$ of cylinder is $9.3 \%$ larger than that in prism. Furthermore, taking $H=1.0$ and $H=$ 1.75 into consideration (Fig. 6.18(a) and Fig. 6.19(a)), $C_{x}, C_{y}$, and $C_{z}$ for prism comprehensively larger than the coefficients in circular cylinder case in majority measurements, and detailed extremum values are summarized in Table 6.2. The extremum values captured from prism are practically higher than those from circular cylinder, and the increasing rates vary from $0.9 \%$ to 19.3\%. This can be interpreted by the shape of the construction. It is known that a larger drag coefficient is always expected when flow passes a blunt body comparing to a streamlined body with no singular point due to the dominance of pressure drag in the case of blunt body. Likewise, square prism in present study is considered as blunt body, while circular cylinder is more like a streamlined obstacle. Thus, the shape discrepancy between prism and cylinder results in the distinction of force coefficients, as shown in Table 6.2. 


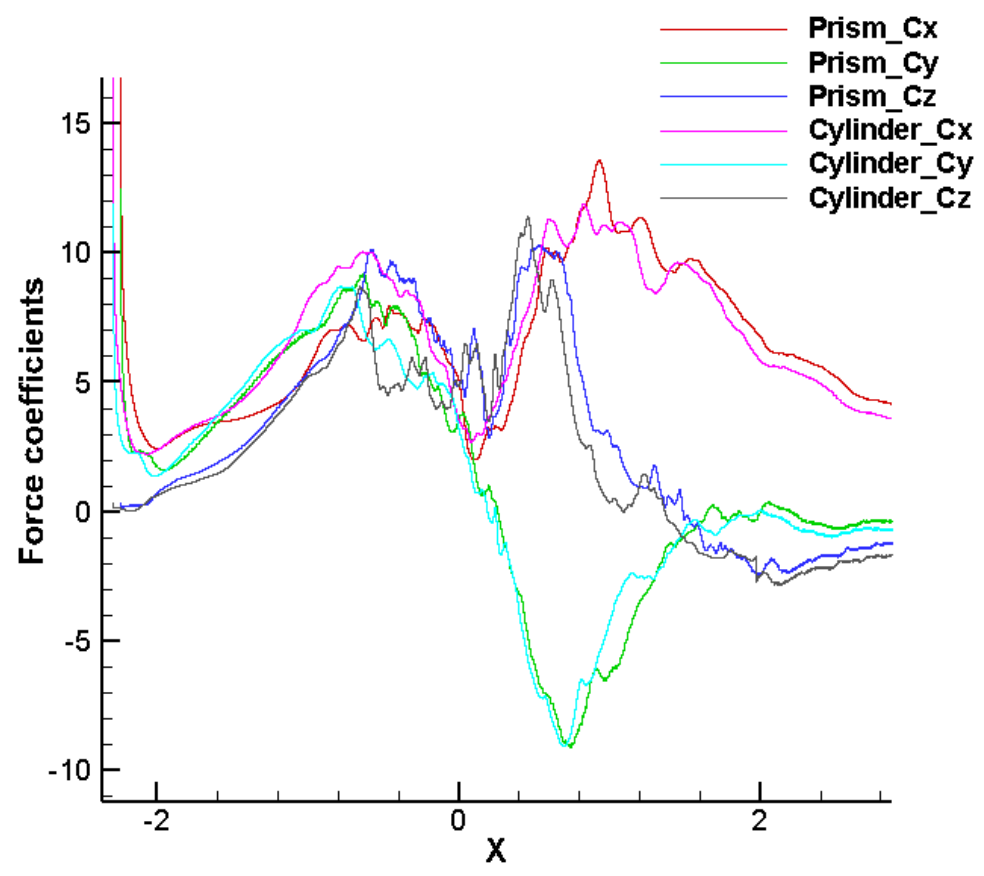

Fig. 6.16 Force coefficient evolutions for different shape at $\mathrm{H}=0.5$

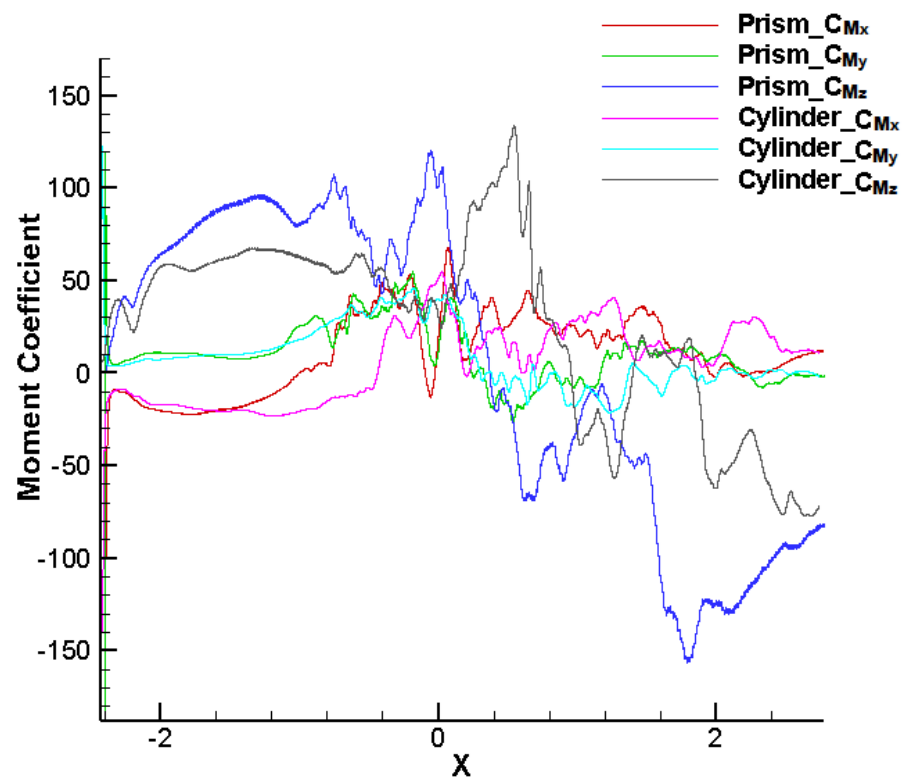

Fig. 6.17 Moment coefficient evolutions for different shape at $\mathrm{H}=0.5$

In Fig. 6.18(b) and Fig. 6.19(b), a detailed look into the moment coefficients comparison between square prism and circular cylinder reveals that the extremums of $C_{M_{x}}, C_{M_{y}}$ and $C_{M_{z}}$ in 175 
different shapes also have diverse performances. One can see that $C_{M_{y}}$ and $C_{M_{z}}$ on prism at different height are much higher than on cylinder at least $16.0 \%$, and up to $86.6 \%$, as listed in

Table 6.3. Recall that $C_{M_{z}}$ is mutually determined by $F_{x}, F_{y}$ and their corresponding force arm, so the higher $C_{M_{z}}$ can be easily interpreted as the loadings on prism are much higher than on cylinder in $x$ - and $y$-direction (Table 6.2). Except for $H=1.75$ case, maximum values of forces and moments obtained from prism at different heights are higher than those on cylinder.

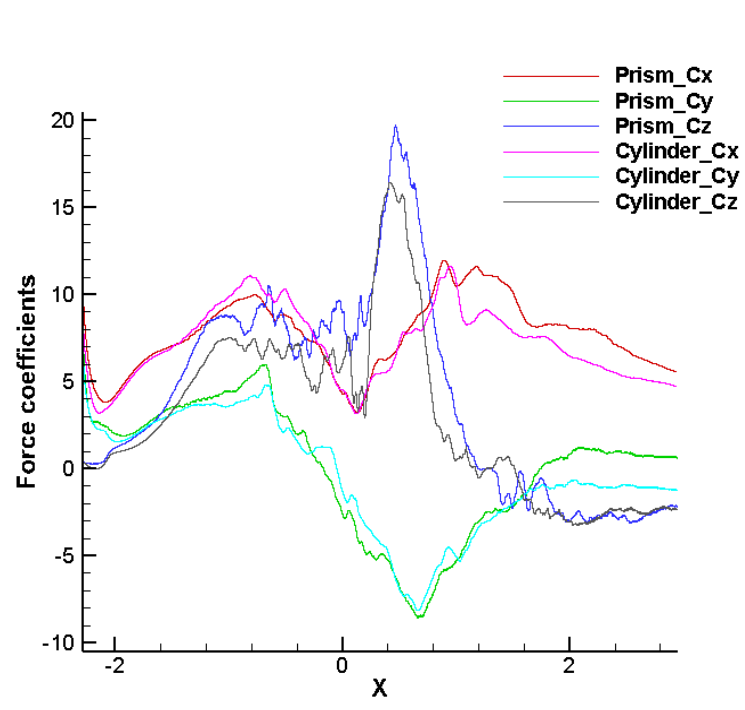

(a)

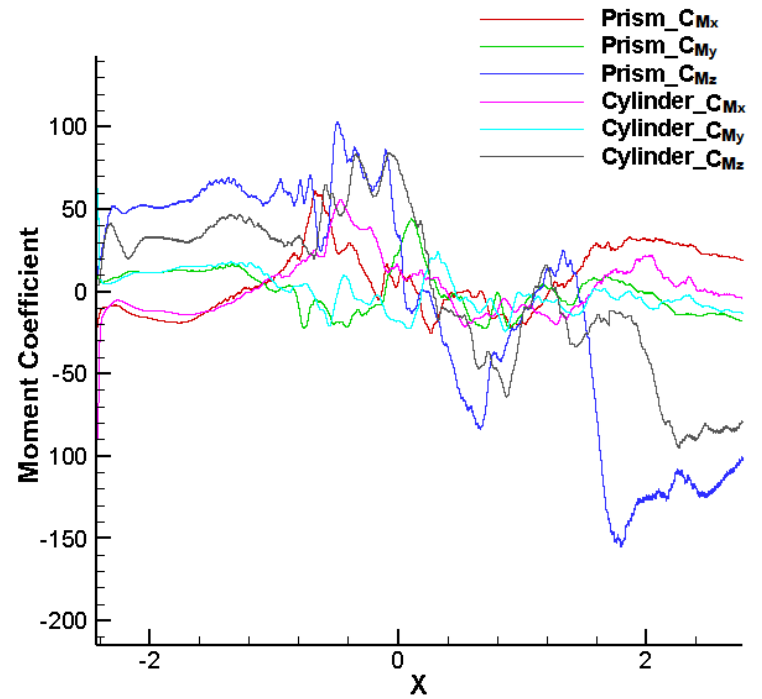

(b)

Fig. 6.18 Force (a) and Moment (b) coefficient evolutions for different shape at $\mathrm{H}=1.0$ 


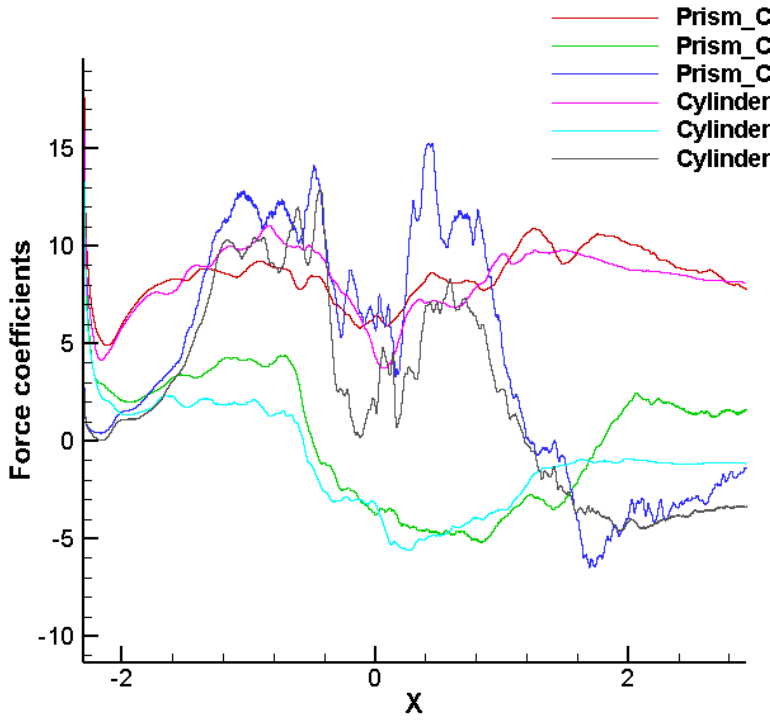

(a)

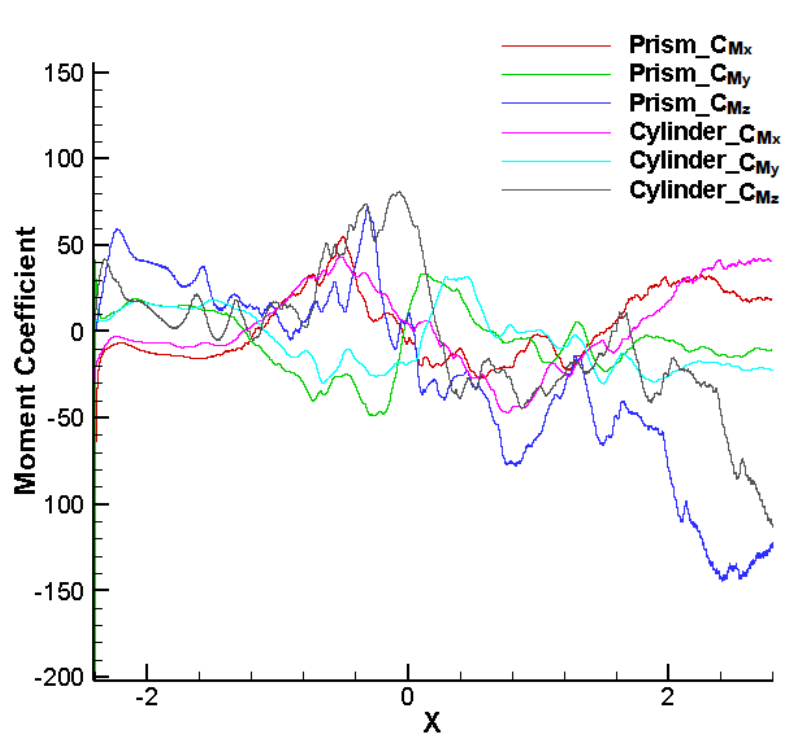

(b)

Fig. 6.19 Force (a) and Moment (b) coefficient evolutions for different shape at $\mathrm{H}=1.75$

Table 6.2 Extremum comparison of force coefficients in different shapes

\begin{tabular}{|c|c|c|c|c|c|c|c|}
\hline \multirow{3}{*}{$H=0.5$} & \multirow{3}{*}{$\begin{array}{l}\text { Cylinder } \\
\text { Prism }\end{array}$} & \multicolumn{2}{|c|}{$\mathrm{C}_{\mathrm{x}}(\max )$} & \multicolumn{2}{|c|}{$\mathrm{C}_{\mathrm{y}}(\max )$} & \multicolumn{2}{|c|}{$\mathrm{C}_{\mathrm{z}}(\max )$} \\
\hline & & 11.85 & 14304 & -9.04 & $0.0 \%$ 个 & 11.39 & $030 \%$ \\
\hline & & 13.54 & $14.5 \%$ & -9.12 & $0.9 \%$ & 10.32 & $9.5 \%$ \\
\hline \multirow[t]{2}{*}{$H=1.0$} & Cylinder & 11.63 & \multirow{2}{*}{$2.9 \%$} & -8.14 & \multirow{2}{*}{$5.3 \% \uparrow$} & 16.44 & \multirow{2}{*}{$19.3 \% \uparrow$} \\
\hline & Prism & 11.97 & & -8.57 & & 19.62 & \\
\hline \multirow[t]{2}{*}{$H=1.75$} & Cylinder & 11.02 & \multirow{2}{*}{$0.8 \%$} & -5.60 & \multirow{2}{*}{$7.0 \% \downarrow$} & 12.83 & \multirow{2}{*}{$18.9 \% \uparrow \uparrow$} \\
\hline & Prism & 10.93 & & -5.21 & & 15.26 & \\
\hline
\end{tabular}

Table 6.3 Extremum comparison of moment coefficients in different shapes

\begin{tabular}{|c|c|c|c|c|c|c|c|}
\hline & & $C_{M_{x}}$ & nax) & $C_{M_{y}}$ & nax) & $C_{M_{z}}$ & ax) \\
\hline \multirow[t]{2}{*}{$H=0.5$} & Cylinder & 54.67 & \multirow{2}{*}{$23.8 \% \uparrow \uparrow$} & 46.33 & \multirow{2}{*}{$18.5 \% \uparrow$} & 132.19 & \multirow{2}{*}{$16.0 \% \uparrow \uparrow$} \\
\hline & Prism & 67.69 & & 54.91 & & -153.37 & \\
\hline \multirow[t]{2}{*}{$H=1.0$} & Cylinder & 55.38 & \multirow{2}{*}{$6.6 \% \uparrow$} & 23.70 & \multirow{2}{*}{$86.6 \% \uparrow$} & -94.81 & \multirow{2}{*}{$63.6 \% \uparrow$} \\
\hline & Prism & 59.06 & & 44.22 & & -155.14 & \\
\hline \multirow[t]{2}{*}{$H=1.75$} & Cylinder & -47.13 & \multirow{2}{*}{$16.5 \% \uparrow$} & 31.75 & \multirow{2}{*}{$52.2 \% \uparrow$} & -111.82 & \multirow{2}{*}{$29.1 \% \uparrow$} \\
\hline & Prism & 54.91 & & -48.31 & & -144.36 & \\
\hline
\end{tabular}


In conclusion, from the mechanic analysis perspective, the building in cylinder shape can be regarded as more effective in resisting the tornado loadings and twisting, than the prism shaped building. The forces and moments can be much reduced when passing structure with no singular point in most of the situations.

\subsubsection{Wind loadings comparison between models with and without prediction-correction technique}

As mentioned in Section 5.4.3 of this study, the proposed immersed boundary method, enhanced by prediction-correction technique has instinctive advantages in numerical accuracy. In this sub-section, the influence of prediction-correction technique on the tornado-induced loadings will be investigated.

Using force coefficient in $x$ - direction, $C_{x}$ as an example, cylinder with four different heights ( $H$ $=0.5,0.75,1.0,1.75$, respectively) will be conducted. All computational parameters remain the same, except for the deactivation of prediction-correction scheme. The $C_{x}$ evolution obtained from framework without prediction-correction scheme is depicted in Fig. 6.20, aiming to compare with existing results in Fig. 6.12(a). As shown in Fig. 6.20, one noticeable difference can be detected near the "core-in" and "coincide" time instants. The local extremum values for all heights near $x=-0.75$ ("core-in" instant) in Fig. 6.20 are far less than those in Fig. 6.12(a). The turning points at "core-in" and "coincide" time instants are inconspicuous as well, comparing to Fig. 6.12(a). The reason can be 
attributed to the implementation of prediction-correction scheme. As previously mentioned, interaction between the tornado and structure should be relatively intensive at the "core-in" and "coincide" time instants. Thus, flow features and force coefficient evolutions in Fig. 6.12(a), are considered to be resolved and captured more practical with prediction-correction technique enforced in the framework. Additionally, in Fig. 6.20, the trends of $C_{x}$ in different heights are similar with each other, in particular at the weak region when tornado has past and departed the structure. However, in Fig. 6.12(a), the slope of $H=1.75$ case is apparently much stable than those from other three heights, at $\mathrm{X}>1.6$. As the ratio of construction height $H$ and domain height is 0.5 , it is not wide enough for flow clearance at the top of the domain with Dirichlet boundary condition applied at the top surface. Under this circumstance, it is believed that the flow might not be fully developed at $H=1.75$, so that the force evolution of this case is reasonably different from other cases with lower constructions. Therefore, the framework with prediction-correction technique enables to capture this characteristic, as illustrated in Fig. 6.12(a). After detailed comparisons, it can be concluded that results obtained from the framework powered by prediction-correction technique are supposed to be more accurate and approximate to the practical scenario. 


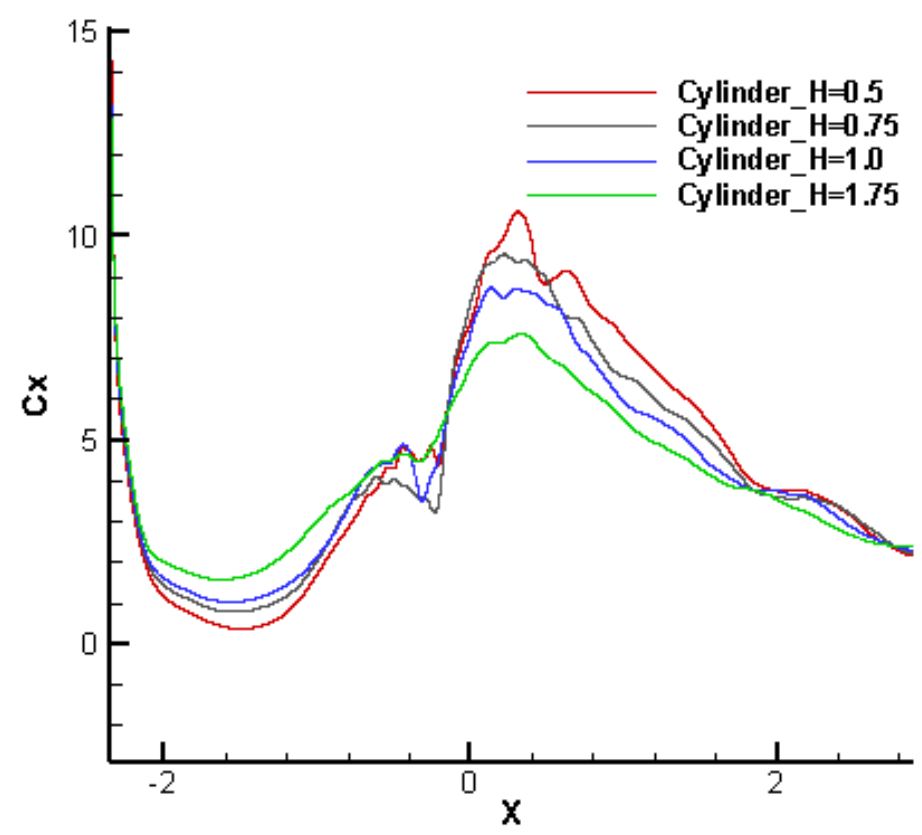

Fig. 6.20 Cx evolutions on cylinder for different heights, without prediction-correction technique

\subsubsection{Comparison between 3D and 2D tornado-induced loadings}

In this section, the results, in particular force coefficients in x- and y- directions $C_{x}$ and $C_{y}$, and moment coefficient $C_{M_{z}}$ obtained from abovementioned 3D scenario are collected by portion, and then compared with those obtained from 2D situation. The objective of this comparison is to provide insights on solving three-dimensional industrial problems in two-dimensional perspective with much lower computational cost, but still valuable and efficient. On the other hand, successfully building the relationship between portions of 3D and 2D cases can further validate the results since the reference regarding the tornado simulation is still insufficient.

The height of construction $H$ is fixed as 1 in the 3D test simulations here. The construction, no 
matter it is a square prism or circular cylinder, is divided into 10 portions from z-plate uniformly, so that each portion has equal height of $H^{\prime}=0.1$. Ten potions are named in sequence as $1 / 10,2 / 10, \ldots$, $10 / 10$, from the bottom to top, respectively. Note that the top plate is treated separately from $10 / 10$ portion, such that the 10 portions have exactly the same shape and same number of Lagrangian points.

The $2 \mathrm{D}$ results are simulated and obtained using the framework proposed in [65]-[66]. The immersed boundary-Lattice Boltzmann Method (IB-LBM) [32][51][65][66][91] is applied as the solver, and detailed solving procedures can be found in [65]-[66]. In order to make 2D domain comparable to the 3D domain discussed aforementioned, parameters are restricted as follows:

- $\quad$ RCVM imposed on the 2D domain satisfies Eq. (6.5) under the condition of $Z_{\mathrm{f}}=1$;

- The ratio of the mesh resolution, as well as diameter of cylinder (D) is the same as the 3D scenario;

- The ratio of domain length $\left(\mathrm{L}_{\mathrm{x}}\right)$ and width $\left(\mathrm{L}_{\mathrm{y}}\right)$, as well as $\mathrm{L}_{\mathrm{x}} / \mathrm{D}$, is the same as the $3 \mathrm{D}$ scenario.

Firstly, results obtained from tornadic wind past cylinder are depicted in Fig. 6.22 - Fig. 6.24. The initial points of $C_{x}$ and $C_{y}$ for each portion in 3D case can be observed starting from $0.3 \sim 0.5$, while $C_{x}$ and $C_{y}$ in 2D (Fig. 6.21) start between 3 and 4, which is around 10 times higher than each portion in 3D case. The reason for the considerable 10 times difference is that thickness of the domain in 2D scenario is regarded to be 1 or a unit, and on the other hand, the thickness of each 
portion of 3D case is 0.1 , regarding to $H=1$ case. Therefore, the initial values of $C_{x}$ and $C_{y}$ for each portion should be approximately 1/10 comparing with 2D simulation, when all other conditions remain the same.

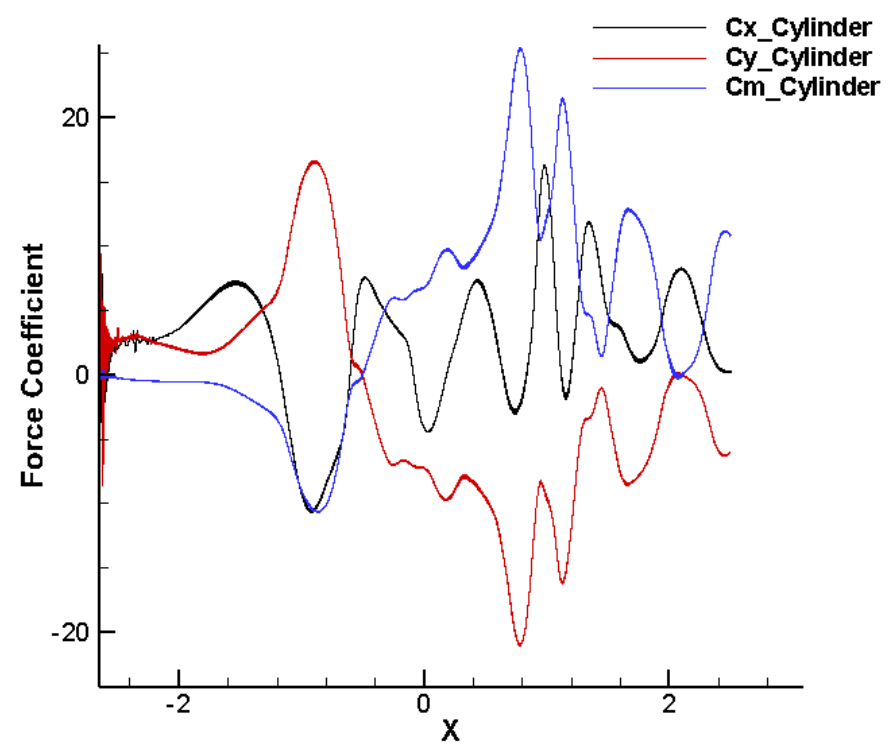

Fig. 6.21 Force coefficients evolution over 2D cylinder scenario

Moreover, the time instance, in which force coefficients reach its peak value can match well with each other. For instance, $C_{x}, C_{y}$ and $C_{m}$ in 2D simulation, as shown in Fig. 6.21, experience violent fluctuation, and reach their respective extremum points near "core-in" and "core-out" time instants. Similarly, same patterns can be observed from Fig. 6.22 - Fig. 6.24 in 3D simulation, regardless of slight time latency for each portion.

To carefully look through the force coefficient curves portion by portion, we can conclude that the curves in $3 / 10$ to $5 / 10$ portions, have the highest similarities comparing with $2 \mathrm{D}$ case. For instance, several noticeable turning points before post-interaction stages can be determined at $x=-1$, 182 
$-0.5,-0.3,0$, and 0.4 in Fig. 6.22 (b) from Portions $3 / 10$ to 5/10. There are four turning points at $x=$ $-1,-0.5,0$, and 0.4 in 2D simulation, which is very similar to what have concluded above. Moreover, $C_{y}$ in Portions 3/10 to 5/10 reaches the first peak value at around $x=-1$, which is earlier than other portions at between $x=-0.8$ to $x=-0.4$. Similarly, the first peak value is observed at $x=-1$ in $2 \mathrm{D}$ case, which matches well with the portions in $3 / 10$ to $5 / 10$. Therefore, it can be concluded that portions $3 / 10$ to $5 / 10$ have the highest similarities comparing to $2 \mathrm{D}$ case, and majority of patterns and trends regarding force and moment coefficients in 2D simulation are still equivalent to those in 3D portions. When considering the portions that close to the bottom surface, the flow characteristics are affected by the ground and boundary conditions, and if the portions are on the top region, three-dimensional effect should be seriously considered. In summary, the middle-range portion is more similar to the flow simulated in 2D scenario.

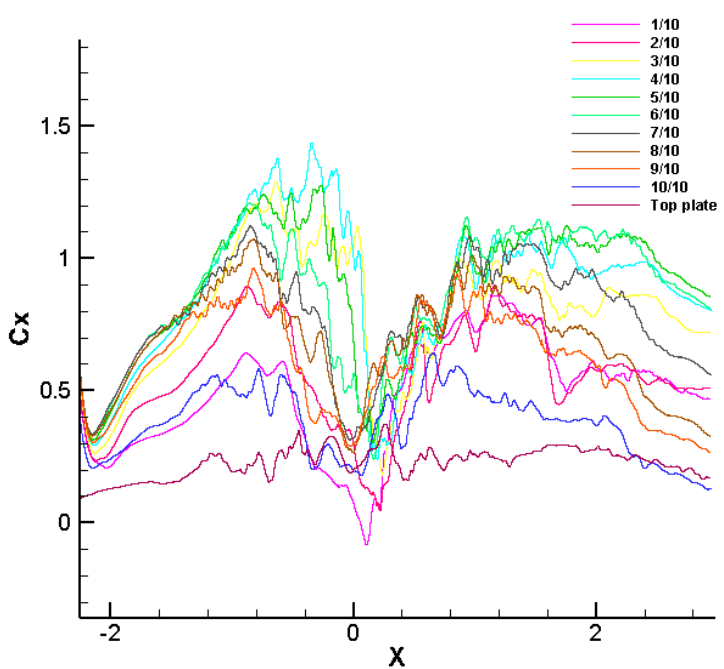

(a)

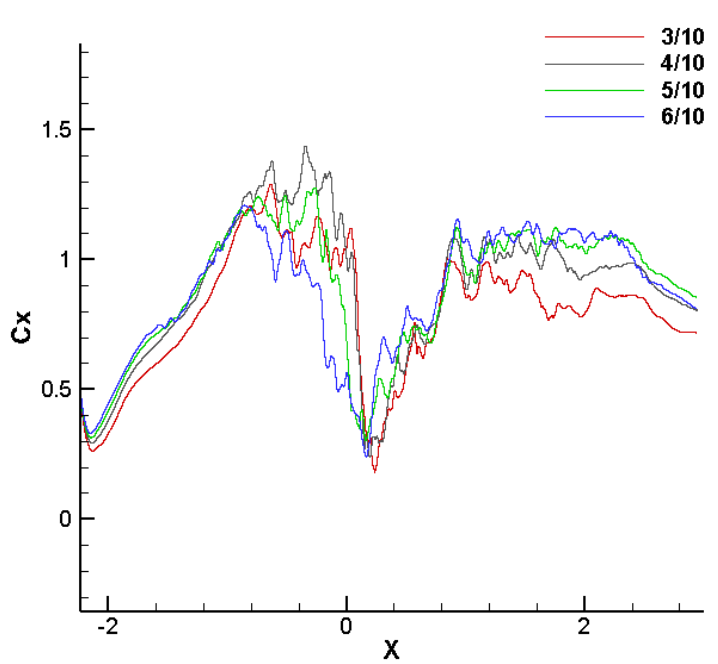

(b)

Fig. 6.22 $\mathrm{C}_{\mathrm{x}}$ evolution for each portion over 3D cylinder scenario: (a). all portions; (b). zoom in to $3 / 10$ to $6 / 10$ portions 


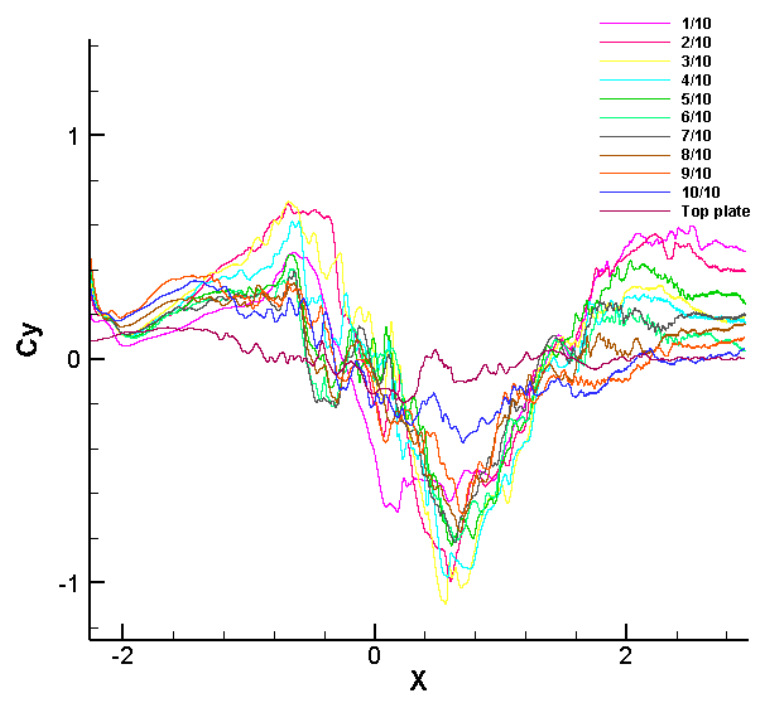

(a)

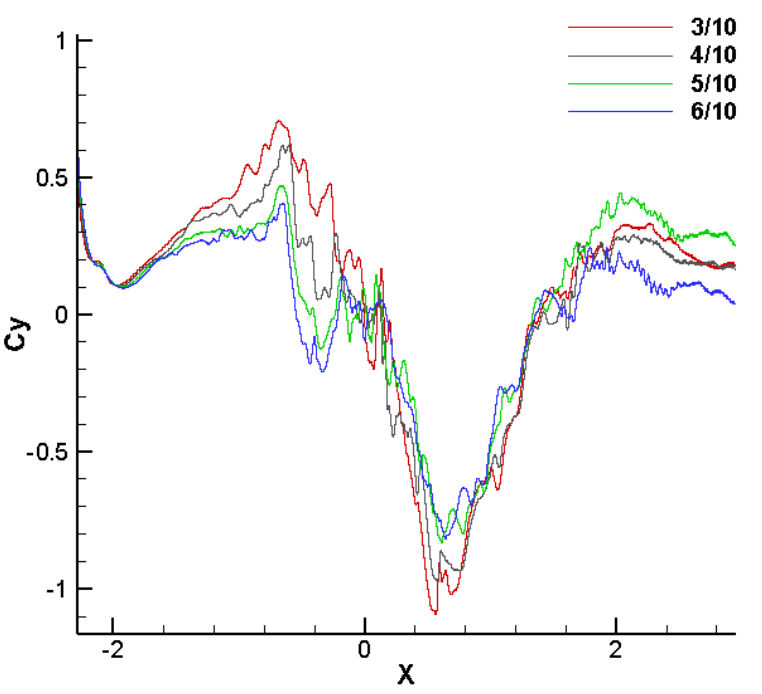

(b)

Fig. 6.23 $\mathrm{C}_{\mathrm{y}}$ evolution for each portion over $3 \mathrm{D}$ cylinder scenario: (a). all portions; (b). zoom in to $3 / 10$ to $6 / 10$ portions

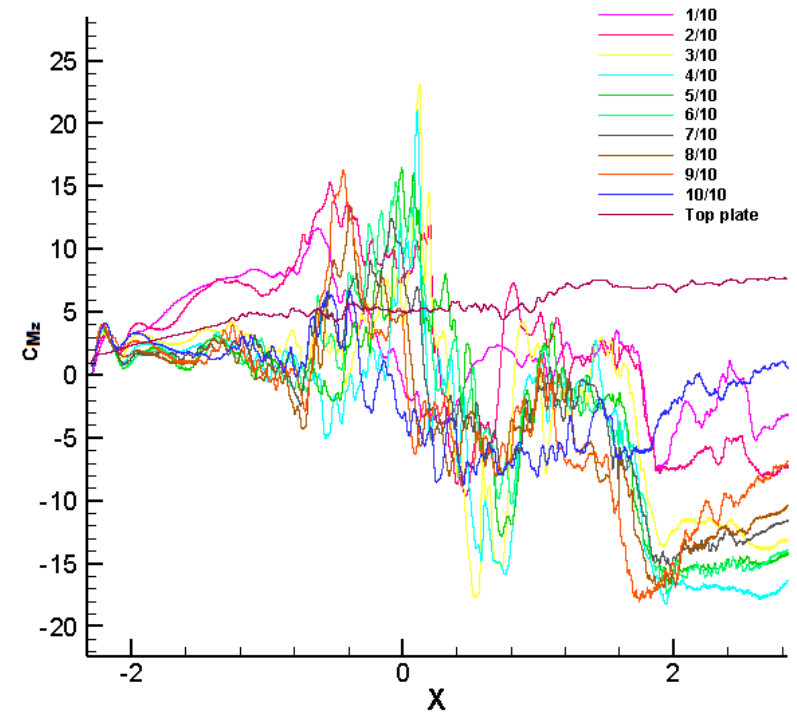

(a)

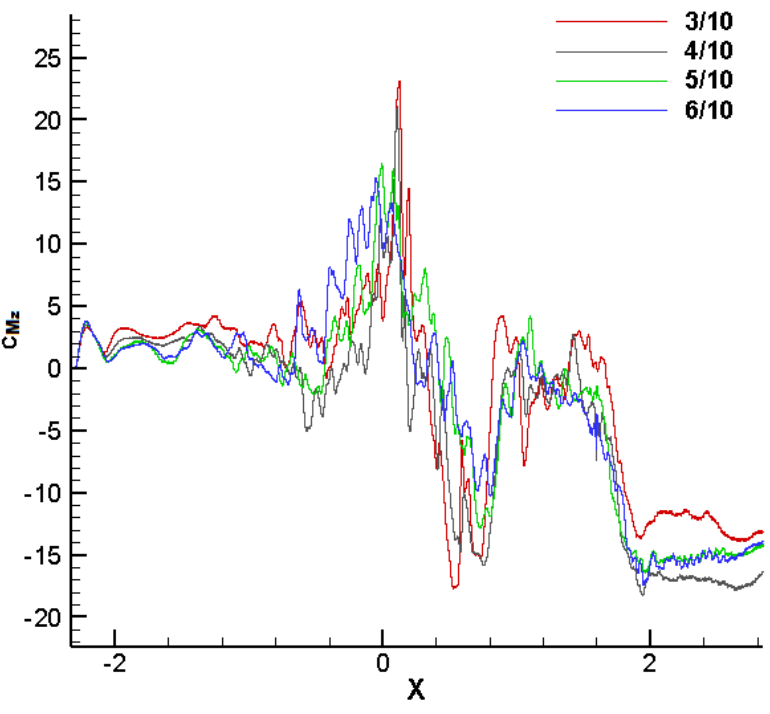

(b)

Fig. 6.24 $\mathrm{C}_{\mathrm{Mz}}$ evolution for each portion over 3D cylinder scenario: (a). all portions; (b). zoom in to $3 / 10$ to $6 / 10$ portions

When it comes to tornado over a square prism case, the observations aforementioned still stand,

as shown in Fig. 6.25 - Fig. 6.28. The evolutions of $C_{x}$ and $C_{y}$ start at around 8 in 2D simulation, which are still about 10 times higher than those starting points of $C_{x}$ and $C_{y}$ measured in 3D domain. 
Again, similar to the discussed in cylinder case, the turning points in both 2D and 3D scenarios are located at the vicinity of $x=-0.7,-0.2,0.2$ and 0.8 , and the trends of $C_{x}, C_{y}$ and $C_{M_{z}}$ are also extraordinarily comparable, in particular the middle portions, such as $3 / 10$ to 5/10.

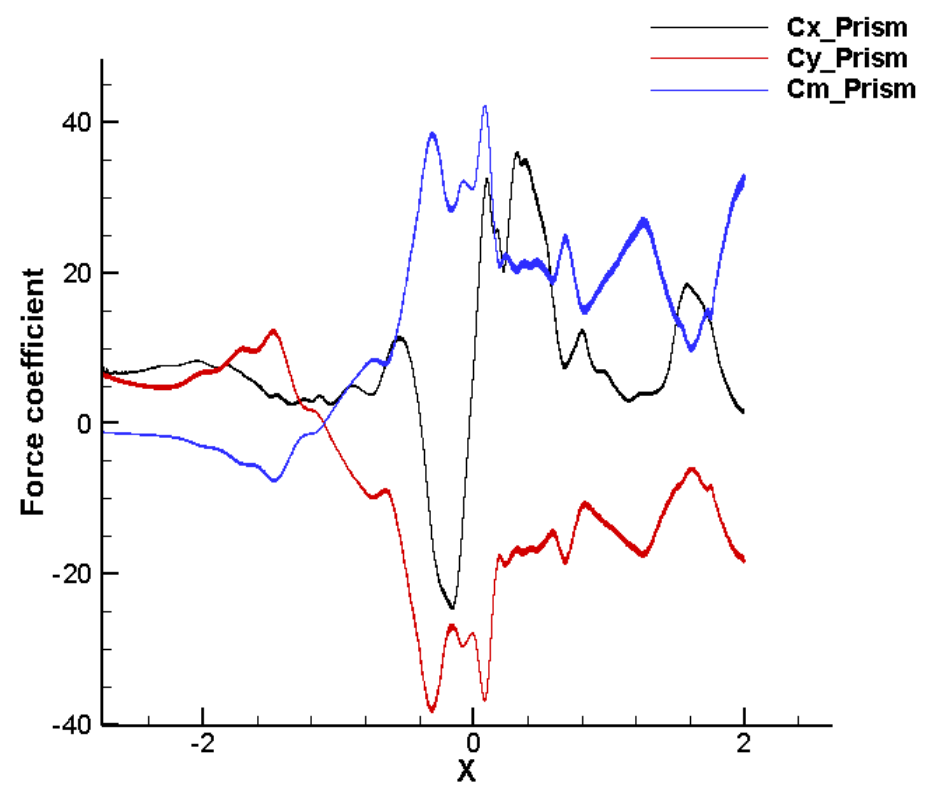

Fig. 6.25 Force coefficients evolution over 2D prism scenario

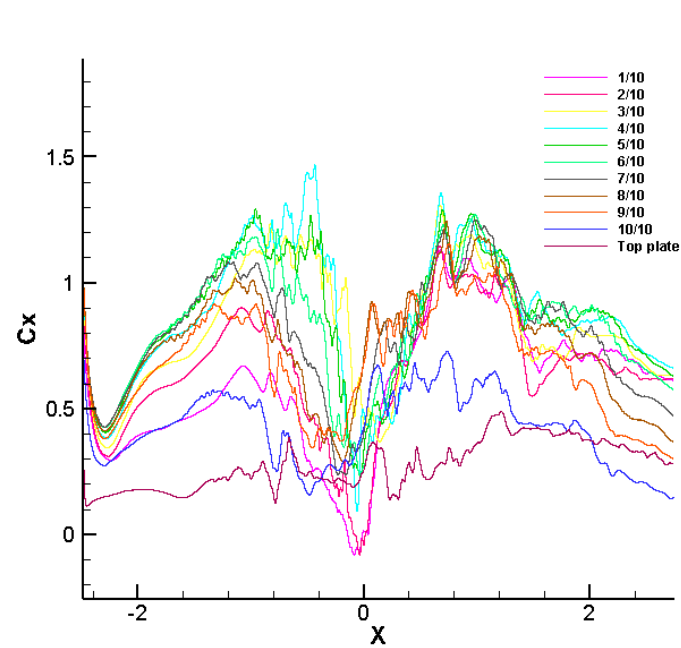

(a)

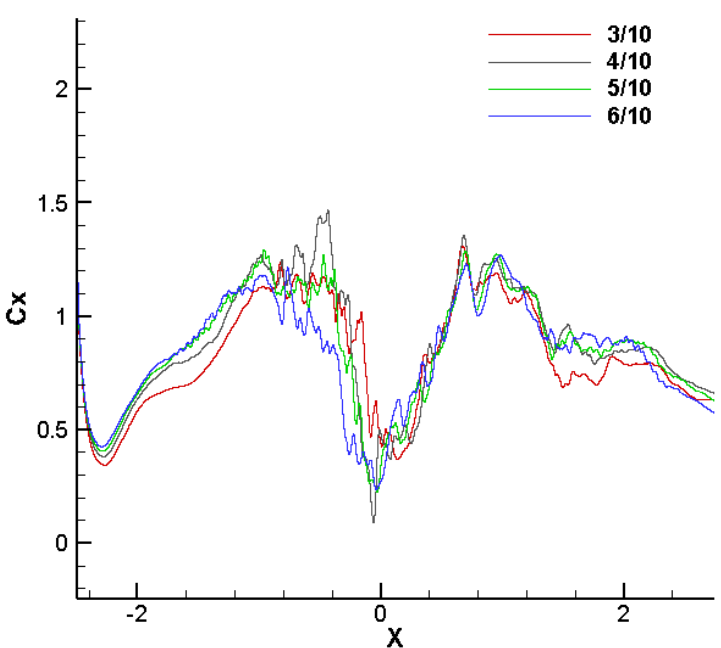

(b)

Fig. $6.26 \mathrm{C}_{\mathrm{x}}$ evolution for each portion over $3 \mathrm{D}$ prism scenario: (a). all portions; (b). zoom in to $3 / 10$ to $6 / 10$ portions 


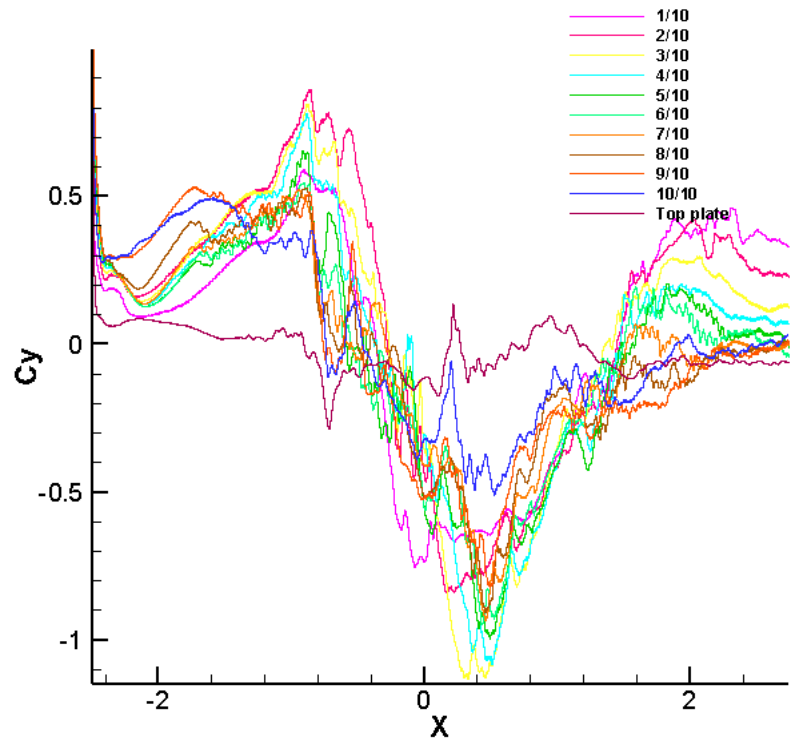

(a)

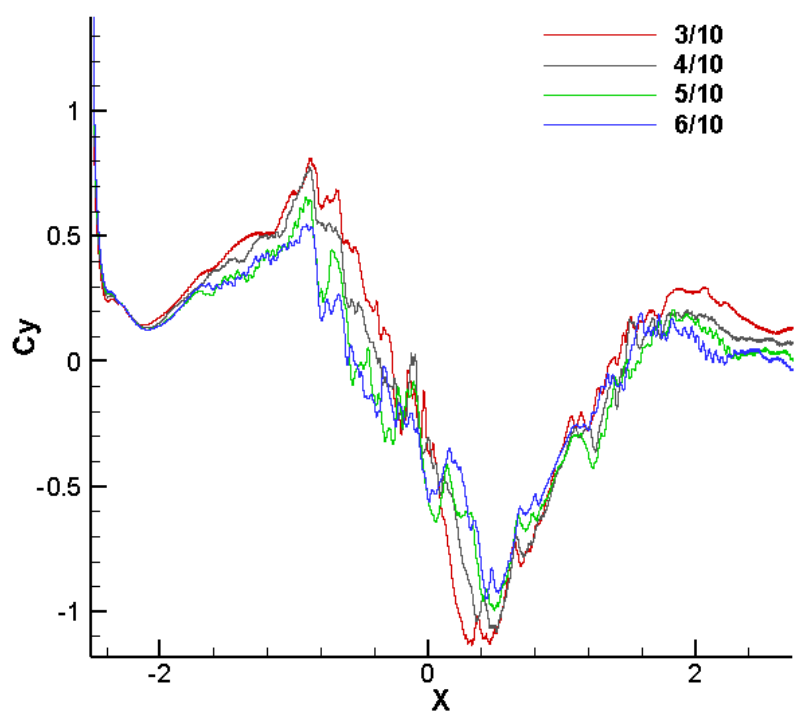

(b)

Fig. 6.27 $\mathrm{C}_{\mathrm{y}}$ evolution for each portion over 3D prism scenario: (a). all portions; (b). zoom in to
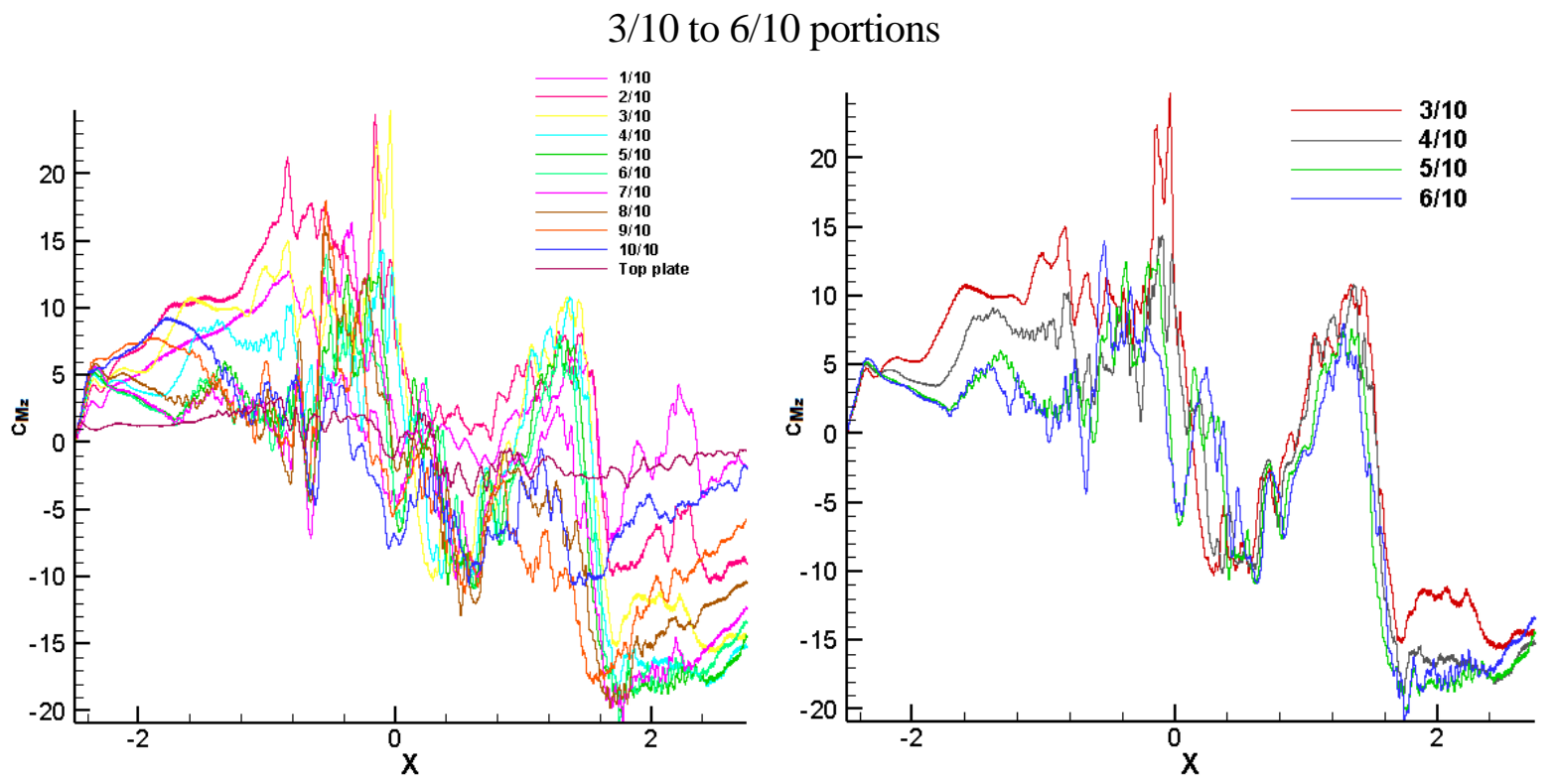

(b)

Fig. 6.28 $\mathrm{C}_{\mathrm{Mz}}$ evolution for each portion over 3D prism scenario: (a). all portions; (b). zoom in to $3 / 10$ to $6 / 10$ portions)

\subsection{Conclusions}

Extended from the first part of this study coupling the feedback forcing based IB with 186 
high-order finite difference based N-S flow solver, this paper focuses on its extensive use particularly aimed at the study of tornado-induced loadings on various ground constructions.

In this paper, the conventional Rankine-Combined Vortex Model (RCVM) is re-tailored so that the immersed boundary approach can be handily applied to investigate the tornado-construction interaction mechanism. In order to extend the previous two-dimensional framework to the three-dimensional version, a logarithm based vertical factor $Z_{f}$ is employed to describe the boundary lay growth on the ground.

The results obtained in the present study reveal that the vertical component of tornado plays a significant role in tornado evolution. The tornado induced force and moment vary significantly in time, and the magnitudes of different components of the force and moment also vary remarkably between each other. Such a crossover of variations renders tremendous complexity to the wind loadings on the buildings, which explains the fundamental reason for the disastrous effects of tornadoes.

In addition, the effects of the tornado forcing on ground construction due to variation of its height and shape were also examined. The findings in this regard are, first, the higher building, the greater loading; second, the circular cylinder-shaped construction generally experiences lower loadings and twists compared to the building of prism shape. In terms of similarity between 2D and 3D-segment simulations, the overall trends for both types of simulations are generally comparable. In particular, the middle-segments (the portions located at the $3 / 10$ to $5 / 10$ height of the entire $3 \mathrm{D}$ 
construction) exhibit more similarities with the $2 \mathrm{D}$ result, since those segments are more distant from the top and bottom boundaries of the simulation domain and, in turn, less influenced by the two boundaries. 


\section{Chapter 7 Conclusions and Future work}

\subsection{Conclusions}

This dissertation mainly focused on developing a set of numerical frameworks to simulate the tornadic winds both in two-dimensional (2D) and three-dimensional (3D) scenarios and investigate the wind loadings and the relative influence factors on a single building and on a multiple-building configuration.

In Chapter 2, a two-dimensional numerical framework is proposed in order to simulate the tornado-building interactions. Here, a hybrid feedback forcing based immersed boundary (IB) lattice Boltzmann method (LBM) is applied for the investigation, after IB-LBM has been proven to be a reliable method in dealing with moving boundary problems by researchers. On the other hand, the conventional Rankine-Combined Vortex Model (RCVM) is customized to be in accordance with the computational setup. The efficacy of the present IB-LBM framework with the customized RCVM is demonstrated through a series of validating cases. After that, the factors that influence the characteristics of tornadic wind are explored. It is concluded that both the translation speed based Reynolds number and the rotation intensity play important roles in altering the tornado characteristics. Particularly, the practitioners have been conventionally using the translational velocity component as the characteristic velocity for defining the Reynolds number, and considering 
such a translation component based Reynolds number as a primary influencing parameter. The present study has found that the translation part based Reynolds number is of one-sided nature for identifying a tornado-like flow, since the rotation is the other part of a tornado and the change of the rotation intensity may strongly alter the loadings exerted on the buildings. Hence, due attention should be also paid on the rotational component in the tornado dynamics research.

In Chapters 3 and 4, the IB-LBM framework is employed to assess different kinds of building configurations (bi-cylinder and a quintuplet-cylinder group) under tornadic winds with deployed input parameters, such as the incoming direction of tornadoes. Except the effect of rotation intensity discussed abovementioned, more discussions are targeted at finding out the inherent reasons that resulted in the loading difference in a building group. Two distinct observations are concluded here: 1) buildings located closer to the tornado translation trajectory are expected to endure greater loadings than those farther away from the trajectory, which is called "front effect" in this work. Therefore, building a resistant wall in front of a building group can be an efficient strategy to prevent building destruction from tornadoes; 2) Tornadic wind center tends to exhibit deviation depending on its rotation direction and, hence, the portions of obstacles located closer to the deviated center will sustain larger wind loadings than the rest of the obstructed objects that are farther from the center. These insights could be helpful for improving design of constructions towards better wind-resistant capabilities.

After a systematical investigation on $2 \mathrm{D}$ tornado scenarios, this study is extended to the $3 \mathrm{D}$ 190 
tornado situation with a logarithm term added to tornadic governing equation representing the effect induced by the vertical velocity component. In Chapter 5, considering the massive computational cost in the three dimensional simulation, the feedback forcing based IB method is incorporated into a Navier-Stokes flow solver with high level parallelizability. Furthermore, a prediction-correction algorithm is embedded into the IB module, which successfully improves both computing efficiency and numerical accuracy at the same time. This novel feedback forcing based framework has been tested extensively throughout a series of 3D benchmark cases, and it is believed that this framework has a solid performance in dealing with moving boundary problems with satisfactory accuracy and computing speed.

Finally, in Chapter 6, the validated framework is applied to examine the tornadic wind loadings over a building in a three-dimensional domain. Vertical component of velocity is found to be dominant, as the force coefficient in vertical direction has the maximum value, which suggests the uprooting effect of devastating tornadoes. This phenomenon is the most significant contribution in 3D simulation, since the vertical effect is not considered in 2D cases. Additionally, it was concluded that constructions with higher height could endure greater loadings and buildings with less singular geometric characteristics suffer less tornadic wind loadings when compared to those in blunt shape.

\subsection{Contributions}

1. Inspired by the success of immersed-boundary (IB) method in dealing with moving/complex 
boundary problems, as well as its conjunction with lattice Boltzmann method (LBM) for a great number of fluid flow applications, a novel IB-LBM framework is first proposed for investigation of tornadic wind effects in two dimensional (2D) scenario.

2. With the aid of "relative motion" principle and IB method, the highly time-dependent tornado boundary condition is re-interpreted by assigning the translational component of tornado to the construction, such that the construction is viewed as "virtually" translating towards a "pinned" rotational flow, resulting in a time-invariant boundary condition. This innovative interpretation for the tornado-building interaction significantly facilitates the establishment of time-independent boundary conditions in such a complex flow.

3. The rotation intensity is proved as the dominant factor that influence the characteristics of a tornadic wind. As indicated in the study, the tornado-induced loadings monotonically increases with the Reynolds number in the lower rotation intensity range, which demonstrates the dominance of translational component in such condition. However, this monotonous no longer exists in larger rotation intensities. At present, the Reynolds number is conventionally only based on the translation velocity component, due attention should be paid to the rotational component as rotation co-plays with the translation part in a $2 \mathrm{D}$ tornado scenario.

4. A series of more practical and complex scenarios, ranging from tornado over bi-cylinders to cylinder-quintuplet with different orientations, are simulated and analyzed in detail in this work. The wind loadings on different structures are also calculated and compared, and the fate of each 
structure can be predicted according to the flow patterns obtained through the numerical simulation. The comparisons also lead to useful insights and guidelines for improving design of constructions towards better wind-resistant capabilities.

5. The RCVM-based tornado model is extended to its three-dimensional version, after taking the vertical velocity component into consideration. Based on an open-source toolkit named Incompact $3 d$, a novel computational framework with a high level of parallelization in the code architecture was developed, which features the feedback forcing based IB model and prediction-correction algorithm. This hybrid framework successfully overcame the numerical oscillation that arises in the simulation of moving/complex boundary problems when using the original Incompact3d. The present framework is fully validated through a series of 3D benchmark cases.

6. The validated feedback forcing based Incompact3d framework is first applied to investigate 3D tornadoes over buildings with different shapes. Comprehensive interpretations, especially the uprooting effect in vertical direction, are provided. Furthermore, the influence factors of wind loadings, and the similarities between 3D and 2D tornado simulations are examined in detail.

\subsection{Recommended future work}

There are still many interesting and important computational tornado dynamics aspects that have not been further explored due to the limit time of this study. The recommendations for the 
future work are as follows:

1. The present study employs uniform Cartesian grids in all simulation cases, which render the mesh resolution insufficient in the sensitive flow zones while excessively fine in the massive portions of the simulation domain where no flow singularity is present. The use of multi-level or, more effectively, adaptive mesh looks particularly beneficial for the future 3D simulations of tornado phenomena.

2. Wind loadings on cylinder was tested and proven to be an optimal option for resisting tornadoes when compared to prism-shaped structures. However, a great variety of other structural geometries, such as dome building, gable roof, hip roof, mansard roof, etc.., have been widely applied in house constructions. Simulations on these building shapes can hopefully provide more practical results in the subject of applied tornado dynamics.

3. The Reynolds number of real-world tornadoes could be much higher than in the scenarios simulated in the present work. In order to investigate the tornado loadings at elevated Reynolds number, a computational domain with a much larger scale is inevitable, which requires more advanced computational and storage facilities. In addition, other powerful techniques regarding turbulence modeling, such as DES (Detached eddy simulation), RANS (Reynolds-averaged Navier-Stokes), DNS (Direct numerical simulation), can be all examined after their incorporation into the current code, and guidelines may be generated on the pertinence of a particular turbulence model in a specific Reynolds number range of tornadoes. 
4. The three-dimensional tornado simulation framework can be also employed to investigate the tornado-induced loadings on more complex and large-scale building configurations, such as the residence community, commercial zone with high rise buildings, and other tangible 3D urban structures. 


\section{Appendix}

\section{A. Unit conversion in LBM [101]}

Using the D2Q9 model [28] as an example, the basic variables in the LBM simulation is length (L), density $(\rho)$, time (t) and kinematic viscosity $(v)$, here all these parameters are in lattice unit. Correspondingly, variables in physical units are defined as: $L_{p}, \rho_{p}, t_{p}, v_{p}$, respectively. In order to convert the variables between lattice unit and physical unit, reference variables are introduced and defined as:

$$
L_{r}=\frac{L_{p}}{L}, \quad \rho_{r}=\frac{\rho_{p}}{\rho}, \quad u_{r}=\frac{u_{p}}{c_{s}}
$$

where $L_{r}, \rho_{r}, u_{r}$ are the reference length, density and velocity. $u_{p}$ is the sound speed in physical unit, and $c_{s}$ is the lattice sound speed. Apart from the relation in length, density and velocity, it is easy to obtain that the time and kinematic viscosity in lattice unit and physical unit satisfy:

$$
\frac{v_{p}}{v}=L_{r} u_{r}, \quad \frac{t_{p}}{t}=\frac{L_{r}}{u_{r}}
$$

Therefore, the aforementioned conventional settings in LBM: $\delta x=\delta y=1, \delta t=1$, and $c_{s}=1 / \sqrt{3}$ can be converted to physical unit as:

$$
\delta x_{p}=\delta y_{p}=L_{r}, \quad \delta t_{p}=\frac{L_{r}}{u_{r}}, \quad u_{p}=\frac{u_{r}}{\sqrt{3}}
$$


Using the tornado case mentioned in Section 2.6.1 as an example: the computational domain is uniformly discretized with $\delta x=\delta y=1$. Physically, the translational velocity of tornado is $20 \mathrm{~m} / \mathrm{s}$, and the diameter of cylinder $L_{p}$ is considered as $20 \mathrm{~m}$. The length of $L_{p}$ is divided into 50 lattices, which means $L_{r}=\frac{20 m}{50}=0.4 m$, and $u_{r}=\frac{340 \mathrm{~m} / \mathrm{s}}{c_{s}}=340 \sqrt{3} \mathrm{~m} / \mathrm{s}$, when considering the sound speed is $340 \mathrm{~m} / \mathrm{s}$ in real world. According to Eq. (A-3), $\delta t_{p}=\frac{L_{r}}{u_{r}}=6.79 \times 10^{-4} \mathrm{~s}$, which corresponding to $\delta t=1$ in lattice unit. At last, the translation velocity in LBM is calculated as $\frac{20}{340 \sqrt{3}}=0.034$, which serves as the characteristic velocity in the simulation. 


\section{References}

[1]. Enhanced Fujita scale for wind damage. Retrived from https://www.canada.ca/en/environment-climate-change/services/seasonal-weather-hazards/enhanced -fujita-scale-wind-damage.html on 2019-06-10

[2]. Cheong, B. L., Palmer, R. D., \& Xue, M. (2008). A time series weather radar simulator based on high-resolution atmospheric models. Journal of Atmospheric and Oceanic Technology, 25(2), 230-243.

[3]. Kurdzo, J. M., Nai, F., Bodine, D. J., Bonin, T. A., Palmer, R. D., Cheong, B. L., \& Byrd, A. D. (2017). Observations of severe local storms and tornadoes with the atmospheric imaging radar. Bulletin of the American Meteorological Society, 98(5), 915-935.

[4]. Yuan, M., Dickens-Micozzi, M., \& Magsig, M. A. (2002). Analysis of tornado damage tracks from the 3 May tornado outbreak using multispectral satellite imagery. Weather and Forecasting, 17(3), 382-398.

[5]. Ward, N. B. (1972). The exploration of certain features of tornado dynamics using a laboratory model. Journal of the Atmospheric Sciences, 29(6), 1194-1204.

[6]. Church, C., Snow, J. T., Baker, G. L., \& Agee, E. M. (1979). Characteristics of tornado-like vortices as a function of swirl ratio: A laboratory investigation. Journal of the Atmospheric Sciences, 36(9), 1755-1776.

[7]. Mishra, A. R., James, D. L., \& Letchford, C. W. (2008). Physical simulation of a single-celled tornado-like vortex, Part B: Wind loading on a cubical model. Journal of Wind Engineering and Industrial Aerodynamics, 96(8-9), 1258-1273.

[8]. Tari, P. H., Gurka, R., \& Hangan, H. (2010). Experimental investigation of tornado-like vortex dynamics with swirl ratio: the mean and turbulent flow fields. Journal of Wind Engineering and Industrial Aerodynamics, 98(12), 936-944.

[9]. Haan Jr, F. L., Sarkar, P. P., \& Gallus, W. A. (2008). Design, construction and performance of a large tornado simulator for wind engineering applications. Engineering Structures, 30(4), 1146-1159.

[10]. Hu, H., Yang, Z., Sarkar, P., \& Haan, F. (2011). Characterization of the wind loads and flow fields around a gable-roof building model in tornado-like winds. Experiments in fluids, 51(3), 835.

[11]. Hangan, H. (2014). The wind engineering energy and environment (WindEEE) dome at western university, Canada. Wind Engineers, JAWE, 39(4), 350-351.

[12]. Refan, M., \& Hangan, H. (2016). Characterization of tornado-like flow fields in a new model scale wind testing chamber. Journal of Wind Engineering and Industrial Aerodynamics, 151, 107-121. 
[13]. Refan, M., Hangan, H., \& Wurman, J. (2014). Reproducing tornadoes in laboratory using proper scaling. Journal of Wind Engineering and Industrial Aerodynamics, 135, 136-148.

[14]. Wilson, T., \& Rotunno, R. (1986). Numerical simulation of a laminar end - wall vortex and boundary layer. The Physics of fluids, 29(12), 3993-4005.

[15]. Nolan, D. S., \& Farrell, B. F. (1999). The structure and dynamics of tornado-like vortices. Journal of the Atmospheric Sciences, 56(16), 2908-2936.

[16]. Lewellen, W. S., Lewellen, D. C., \& Sykes, R. I. (1997). Large-eddy simulation of a tornado's interaction with the surface. Journal of the Atmospheric Sciences, 54(5), 581-605.

[17]. Lewellen, D. C., \& Lewellen, W. S. (2007). Near-surface intensification of tornado vortices. Journal of the Atmospheric Sciences, 64(7), 2176-2194.

[18]. Ishihara, T., Oh, S., \& Tokuyama, Y. (2011). Numerical study on flow fields of tornado-like vortices using the LES turbulence model. Journal of Wind Engineering and Industrial Aerodynamics, 99(4), 239-248.

[19]. Selvam, R. P. (1997). Computation of pressures on Texas Tech University building using large eddy simulation. Journal of Wind Engineering and Industrial Aerodynamics, 67, 647-657.

[20]. Selvam, R. P., \& Millett, P. C. (2003). Computer modeling of tornado forces on buildings. Wind and Structures, 6(3), 209-220.

[21]. Gorecki, P., \& Selvam, R. P. (2015). Rankine combined vortex interaction with a rectangular prism. International Journal of Computational Fluid Dynamics, 29(1), 120-132.

[22]. Smagorinsky, J. (1963). General circulation experiments with the primitive equations: I. The basic experiment. Monthly weather review, 91(3), 99-164.

[23]. Kuai, L., Haan Jr, F. L., Gallus Jr, W. A., \& Sarkar, P. P. (2008). CFD simulations of the flow field of a laboratory-simulated tornado for parameter sensitivity studies and comparison with field measurements. Wind and Structures, 11(2), 75-96.

[24]. Cao, S., Wang, M., \& Cao, J. (2018). Numerical study of wind pressure on low-rise buildings induced by tornado-like flows. Journal of Wind Engineering and Industrial Aerodynamics, 183, 214-222.

[25]. Rotunno, R. (2013). The fluid dynamics of tornadoes. Annual review of fluid mechanics, 45, 59-84.

[26]. Bienkiewicz, B. (1993). Physical modeling of tornado-like flow and tornado effects on building loading. In Proceeding of 7th US national conference on wind engineering, 1993 (pp. 95-106).

[27]. Aidun, C. K., \& Clausen, J. R. (2010). Lattice-Boltzmann method for complex flows. Annual review of fluid mechanics, 42, 439-472.

[28]. Qian, Y. H., d'Humières, D., \& Lallemand, P. (1992). Lattice BGK models for Navier-Stokes equation. EPL (Europhysics Letters), 17(6), 479.

[29]. Zhang, J. (2011). Lattice Boltzmann method for microfluidics: models and applications. 
Microfluidics and Nanofluidics, 10(1), 1-28.

[30]. Peskin, C. S. (1972). Flow patterns around heart valves: a numerical method. Journal of computational physics, 10(2), 252-271.

[31]. Peskin, C. S. (2002). The immersed boundary method. Acta numerica, 11, 479-517.

[32]. Feng, Z. G., \& Michaelides, E. E. (2004). The immersed boundary-lattice Boltzmann method for solving fluid-particles interaction problems. Journal of Computational Physics, 195(2), 602-628.

[33]. Feng, Z. G., \& Michaelides, E. E. (2005). Proteus: a direct forcing method in the simulations of particulate flows. Journal of Computational Physics, 202(1), 20-51.

[34]. Wu, J., Qiu, Y. L., Shu, C., Zhao, N., \& Wang, X. (2015). An adaptive immersed boundary-lattice Boltzmann method for simulating a flapping foil in ground effect. Computers \& Fluids, 106, 171-184.

[35]. Wu, J., Liu, C., Yang, S. C., \& Zhao, N. (2015). Influence of a flexible tail on the performance of a foil hovering near the ground: numerical investigation. European Journal of Mechanics-B/Fluids, 52, 85-96.

[36]. Tian, F. B., Luo, H., Zhu, L., Liao, J. C., \& Lu, X. Y. (2011). An efficient immersed boundary-lattice Boltzmann method for the hydrodynamic interaction of elastic filaments. Journal of computational physics, 230(19), 7266-7283.

[37]. Zhang, H., Tan, Y., Shu, S., Niu, X., Trias, F. X., Yang, D., .. \& Sheng, Y. (2014). Numerical investigation on the role of discrete element method in combined LBM-IBM-DEM modeling. Computers \& Fluids, 94, 37-48.

[38]. Huang, R., \& Wu, H. (2014). An immersed boundary-thermal lattice Boltzmann method for solid-liquid phase change. Journal of Computational Physics, 277, 305-319.

[39]. Lallemand, P., \& Luo, L. S. (2000). Theory of the lattice Boltzmann method: Dispersion, dissipation, isotropy, Galilean invariance, and stability. Physical Review E, 61(6), 6546.

[40]. Guo, Z., Zheng, C., \& Shi, B. (2002). Discrete lattice effects on the forcing term in the lattice Boltzmann method. Physical Review E, 65(4), 046308.

[41]. Goldstein, D., Handler, R., \& Sirovich, L. (1993). Modeling a no-slip flow boundary with an external force field. Journal of computational physics, 105(2), 354-366.

[42]. Pozrikidis, C., \& Ferziger, J. H. (1997). Introduction to theoretical and computational fluid dynamics. Physics Today, 50, 72.

[43]. Wanderley, J. B., Souza, G. H., Sphaier, S. H., \& Levi, C. (2008). Vortex-induced vibration of an elastically mounted circular cylinder using an upwind TVD two-dimensional numerical scheme. Ocean Engineering, 35(14-15), 1533-1544.

[44]. Cao, S., Ozono, S., Tamura, Y., Ge, Y., \& Kikugawa, H. (2010). Numerical simulation of Reynolds number effects on velocity shear flow around a circular cylinder. Journal of Fluids and structures, 26(5), 685-702. 
[45]. Lecointe, Y., \& Piquet, J. (1989). Flow structure in the wake of an oscillating cylinder. Journal of fluids engineering, 111(2), 139-148.

[46]. Beaudan, P., \& Moin, P. (1994). Numerical experiments on the flow past a circular cylinder at sub-critical Reynolds number (No. TF-62). STANFORD UNIV CA THERMOSCIENCES DIV.

[47]. Breuer, M. (1998). Numerical and modeling influences on large eddy simulations for the flow past a circular cylinder. International Journal of Heat and Fluid Flow, 19(5), 512-521.

[48]. Kravchenko, A. G., \& Moin, P. (2000). Numerical studies of flow over a circular cylinder at $\operatorname{Re} \mathrm{D}=3900$. Physics of fluids, 12(2), 403-417.

[49]. Guo, X., Yao, J., Zhong, C., \& Cao, J. (2015). A hybrid adaptive-gridding immersed-boundary lattice Boltzmann method for viscous flow simulations. Applied Mathematics and Computation, 267, 529-553.

[50]. Yuan, R., Zhong, C., \& Zhang, H. (2015). An immersed-boundary method based on the gas kinetic BGK scheme for incompressible viscous flow. Journal of Computational Physics, 296, 184-208.

[51]. Wu, J., Shu, C., \& Zhang, Y. H. (2010). Simulation of incompressible viscous flows around moving objects by a variant of immersed boundary - lattice Boltzmann method. International Journal for Numerical Methods in Fluids, 62(3), 327-354.

[52]. Choi, S., Choi, H., \& Kang, S. (2002). Characteristics of flow over a rotationally oscillating cylinder at low Reynolds number. Physics of Fluids, 14(8), 2767-2777.

[53]. Succi, S. (2001). The lattice Boltzmann equation: for fluid dynamics and beyond. Oxford university press.

[54]. Zhu, L., He, G., Wang, S., Miller, L., Zhang, X., You, Q., \& Fang, S. (2011). An immersed boundary method based on the lattice Boltzmann approach in three dimensions, with application. Computers \& Mathematics with Applications, 61(12), 3506-3518.

[55]. Wu, J., \& Shu, C. (2010). An improved immersed boundary-lattice Boltzmann method for simulating three-dimensional incompressible flows. Journal of Computational Physics, 229(13), 5022-5042.

[56]. Yang, Z., Sarkar, P., \& Hu, H. (2011). An experimental study of a high-rise building model in tornado-like winds. Journal of Fluids and Structures, 27(4), 471-486.

[57]. Chen, S., \& Doolen, G. D. (1998). Lattice Boltzmann method for fluid flows. Annual review of fluid mechanics, 30(1), 329-364.

[58]. Hou, S., Zou, Q., Chen, S., Doolen, G., \& Cogley, A. C. (1995). Simulation of cavity flow by the lattice Boltzmann method. Journal of computational physics, 118(2), 329-347.

[59]. Dupuis, A., Chatelain, P., \& Koumoutsakos, P. (2008). An immersed boundary-lattice-Boltzmann method for the simulation of the flow past an impulsively started cylinder. Journal of Computational Physics, 227(9), 4486-4498.

[60]. Wu, J., \& Shu, C. (2009). Implicit velocity correction-based immersed boundary-lattice 
Boltzmann method and its applications. Journal of Computational Physics, 228(6), 1963-1979.

[61]. Hirt, C. W., Amsden, A. A., \& Cook, J. L. (1974). An arbitrary Lagrangian-Eulerian computing method for all flow speeds. Journal of computational physics, 14(3), 227-253.

[62]. Anderson, R. W., Elliott, N. S., \& Pember, R. B. (2004). An arbitrary Lagrangian-Eulerian method with adaptive mesh refinement for the solution of the Euler equations. Journal of Computational Physics, 199(2), 598-617.

[63]. Glowinski, R., Pan, T. W., Hesla, T. I., \& Joseph, D. D. (1999). A distributed Lagrange multiplier/fictitious domain method for particulate flows. International Journal of Multiphase Flow, 25(5), 755-794.

[64]. Patankar, N. A., Singh, P., Joseph, D. D., Glowinski, R., \& Pan, T. W. (2000). A new formulation of the distributed Lagrange multiplier/fictitious domain method for particulate flows. International Journal of Multiphase Flow, 26(9), 1509-1524.

[65]. Guo, X., \& Cao, J. (2016). An IB-LBM investigation into the aerodynamic coefficients in relation to the rotation intensity of a tornado-like wind. Computers \& Mathematics with Applications.

[66]. Guo, X., Palanisamy, R., \& Cao, J. (2017). A two-dimensional IB-LBM framework combined with re-tailored RCVM for assessing the rotation intensity of a tornadic wind over a building configuration. Engineering Structures, 131, 57-68.

[67]. Liu, Z., \& Ishihara, T. (2015). Numerical study of turbulent flow fields and the similarity of tornado vortices using large-eddy simulations. Journal of Wind Engineering and Industrial Aerodynamics, 145, 42-60.

[68]. Liu, Z., \& Ishihara, T. (2015). A study of tornado induced mean aerodynamic forces on a gable-roofed building by the large eddy simulations. Journal of Wind Engineering and Industrial Aerodynamics, 146, 39-50.

[69]. Laizet, S., \& Lamballais, E. (2009). High-order compact schemes for incompressible flows: A simple and efficient method with quasi-spectral accuracy. Journal of Computational Physics, 228(16), 5989-6015.

[70]. Lamballais, E., Fortuné, V., \& Laizet, S. (2011). Straightforward high-order numerical dissipation via the viscous term for direct and large eddy simulation. Journal of Computational Physics, 230(9), 3270-3275.

[71]. Piomelli, U., Moin, P., \& Ferziger, J. H. (1988). Model consistency in large eddy simulation of turbulent channel flows. The Physics of fluids, 31(7), 1884-1891.

[72]. Moser, R. D., Kim, J., \& Mansour, N. N. (1999). Direct numerical simulation of turbulent channel flow up to Re $\tau=590$. Physics of fluids, 11(4), 943-945.

[73]. Mohd-Yusof, J. (1997). Combined immersed boundaries/B-splines methods for simulations of flows in complex geometries. CTR Annual Research Briefs, NASA Ames.

[74]. Fadlun, E. A., Verzicco, R., Orlandi, P., \& Mohd-Yusof, J. (2000). Combined 
immersed-boundary finite-difference methods for three-dimensional complex flow simulations. Journal of computational physics, 161(1), 35-60.

[75]. Yang, J., Preidikman, S., \& Balaras, E. (2008). A strongly coupled, embedded-boundary method for fluid-structure interactions of elastically mounted rigid bodies. Journal of Fluids and Structures, 24(2), 167-182.

[76]. Taira, K., \& Colonius, T. (2007). The immersed boundary method: a projection approach. Journal of Computational Physics, 225(2), 2118-2137.

[77]. Zeng, X., \& Farhat, C. (2012). A systematic approach for constructing higher-order immersed boundary and ghost fluid methods for fluid-structure interaction problems. Journal of Computational Physics, 231(7), 2892-2923.

[78]. Seo, J. H., \& Mittal, R. (2011). A high-order immersed boundary method for acoustic wave scattering and low-Mach number flow-induced sound in complex geometries. Journal of computational physics, 230(4), 1000-1019.

[79]. Yang, J., \& Balaras, E. (2006). An embedded-boundary formulation for large-eddy simulation of turbulent flows interacting with moving boundaries. Journal of Computational Physics, 215(1), 12-40.

[80]. Linnick, M. N., \& Fasel, H. F. (2005). A high-order immersed interface method for simulating unsteady incompressible flows on irregular domains. Journal of Computational Physics, 204(1), 157-192.

[81]. Laizet, S., \& Li, N. (2011). Incompact3d: A powerful tool to tackle turbulence problems with up to $\mathrm{O}$ (105) computational cores. International Journal for Numerical Methods in Fluids, 67(11), 1735-1757.

[82]. Lele, S. K. (1992). Compact finite difference schemes with spectral-like resolution. Journal of computational physics, 103(1), 16-42.

[83]. Kim, J., \& Moin, P. (1985). Application of a fractional-step method to incompressible Navier-Stokes equations. Journal of computational physics, 59(2), 308-323.

[84]. Chang, W., Giraldo, F., \& Perot, B. (2002). Analysis of an exact fractional step method. Journal of Computational Physics, 180(1), 183-199.

[85]. Parnaudeau, P., Carlier, J., Heitz, D., \& Lamballais, E. (2008). Experimental and numerical studies of the flow over a circular cylinder at Reynolds number 3900. Physics of Fluids, 20(8), 085101.

[86]. Parnaudeau, P., Lamballais, E., Heitz, D., \& Silvestrini, J. H. (2004). Combination of the immersed boundary method with compact schemes for DNS of flows in complex geometry. In Direct and Large-Eddy Simulation V (pp. 581-590). Springer, Dordrecht.

[87]. Cao, J., \& Kitanidis, P. K. (2007). An algorithm for solving reactive advection-dispersion problems. International Journal for Computational Methods in Engineering Science and Mechanics, 8(2), 89-102. 
[88]. Schlichting, H., \& Gersten, K. (2016). Boundary-layer theory. Springer.

[89]. Fornberg, B. (1980). A numerical study of steady viscous flow past a circular cylinder. Journal of Fluid Mechanics, 98(4), 819-855.

[90]. Shukla, R. K., Tatineni, M., \& Zhong, X. (2007). Very high-order compact finite difference schemes on non-uniform grids for incompressible Navier-Stokes equations. Journal of Computational Physics, 224(2), 1064-1094.

[91]. Niu, X. D., Shu, C., Chew, Y. T., \& Peng, Y. (2006). A momentum exchange-based immersed boundary-lattice Boltzmann method for simulating incompressible viscous flows. Physics Letters A, 354(3), 173-182.

[92]. Saiki, E. M., \& Biringen, S. (1996). Numerical simulation of a cylinder in uniform flow: application of a virtual boundary method. Journal of Computational Physics, 123(2), 450-465.

[93]. Silva, A. L. E., Silveira-Neto, A., \& Damasceno, J. J. R. (2003). Numerical simulation of two-dimensional flows over a circular cylinder using the immersed boundary method. Journal of Computational Physics, 189(2), 351-370.

[94]. Russell, D., \& Wang, Z. J. (2003). A Cartesian grid method for modeling multiple moving objects in 2D incompressible viscous flow. Journal of Computational Physics, 191(1), 177-205.

[95]. Roache, P. J. (1994). Perspective: a method for uniform reporting of grid refinement studies. Journal of Fluids Engineering, 116(3), 405-413.

[96]. Rastan, M. R., Sohankar, A., \& Alam, M. M. (2017). Low-Reynolds-number flow around a wall-mounted square cylinder: Flow structures and onset of vortex shedding. Physics of Fluids, 29(10), 103601.

[97]. Zhao, M., Cheng, L., \& Zhou, T. (2009). Direct numerical simulation of three-dimensional flow past a yawed circular cylinder of infinite length. Journal of Fluids and Structures, 25(5), 831-847.

[98]. Lei, C., Cheng, L., \& Kavanagh, K. (2001). Spanwise length effects on three-dimensional modelling of flow over a circular cylinder. Computer methods in applied mechanics and engineering, 190(22-23), 2909-2923.

[99]. Mittal, S. (2001). Computation of three-dimensional flows past circular cylinder of low aspect ratio. Physics of Fluids, 13(1), 177-191.

[100]. Akins, R. E., Peterka, J. A., \& Cermak, J. E. (1977). Mean force and moment coefficients for buildings in turbulent boundary layers. Journal of Wind Engineering and Industrial Aerodynamics, 2(3), 195-209.

[101]. Succi, S. (2001). The lattice Boltzmann equation: for fluid dynamics and beyond. Oxford university press. 